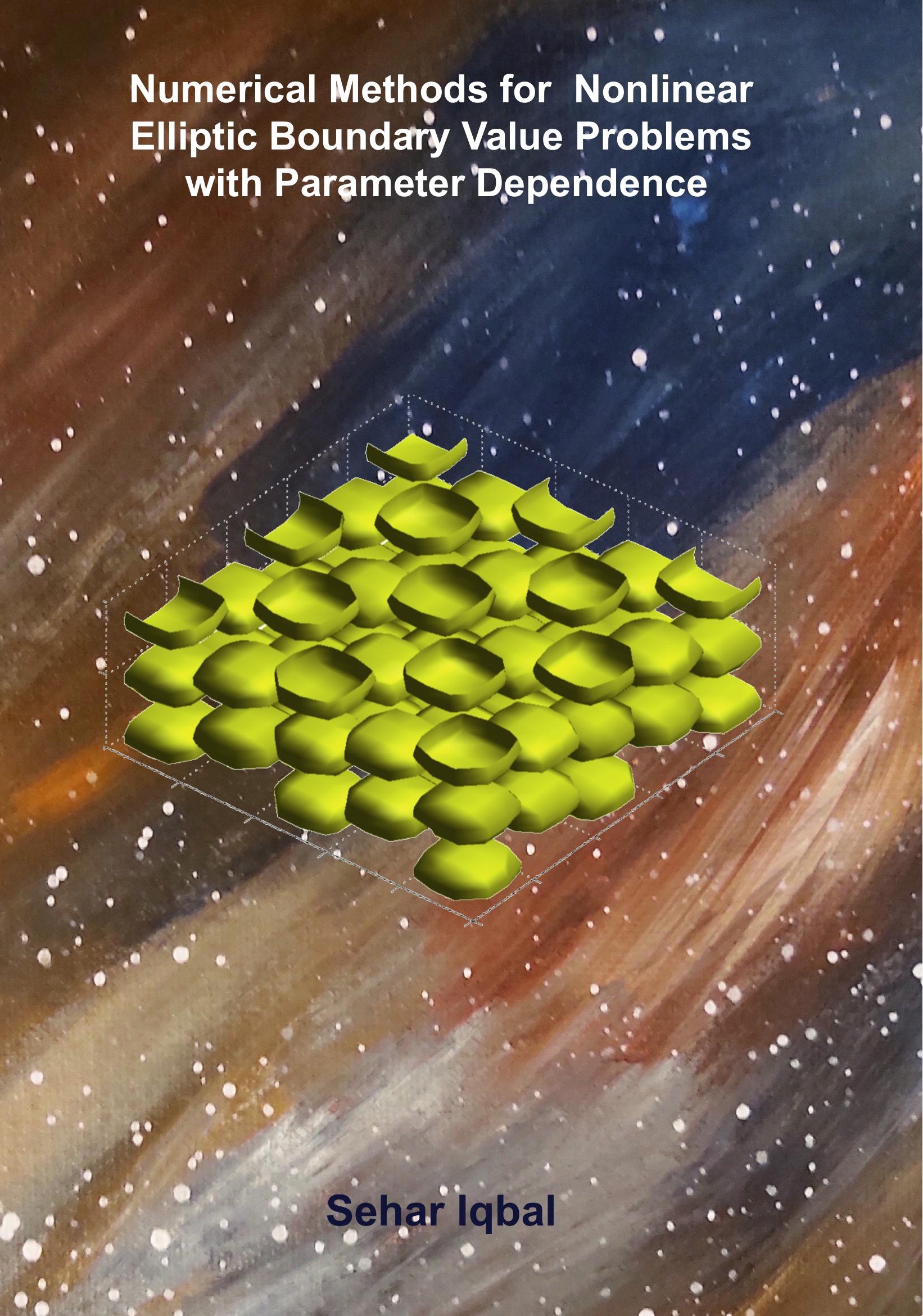


Numerical Methods for Nonlinear Elliptic Boundary Value Problems with Parameter Dependence 
Thesis committee:

Prof. dr. C. J. Budd, University of Bath

Prof. dr. C. Rohde, University of Stuttgart

Prof. dr. ir. B. Koren, Eindhoven University of Technology

Prof. dr. ir. C. W. Oosterlee, Delft University of Technology

Dr. L. Kamenski, m4sim GmbH Berlin

ISBN: 978-90-393-7240-1

Copyright (0) 2020 by Sehar Iqbal. All rights reserved.

Cover: The cover page is an abstract art painted by myself especially for my thesis. The inspiration for this abstract image is from Max Tegmark's view, everything in the universe - humans included - is part of a mathematical structure. The image on the frontside is the iso-surface visualization of the periodic solution for the three-dimensional Gelfand-Bratu model. 


\title{
Numerical Methods for Nonlinear Elliptic Boundary Value Problems with Parameter Dependence
}

\author{
Numerieke methoden voor niet-lineaire elliptische \\ randwaardeproblemen met parameter-afhankelijkheid \\ (met een samenvatting in het Nederlands)
}

\section{Proefschrift}

ter verkrijging van de graad van doctor aan de Universiteit Utrecht op gezag van de rector magnificus, prof. dr. H.R.B.M. Kummeling, ingevolge het besluit van het college voor promoties in het openbaar te verdedigen op maandag 20 januari 2020 des ochtends te 10.30 uur

door

\section{Sehar Iqbal}

geboren op 29 november 1983 te Faisalabad, Pakistan 
Promotor: $\quad$ Prof.dr. R.H. Bisseling

Copromotor: Dr. P. A. Zegeling

This thesis was accomplished with financial support from Schlumberger Foundation (faculty for the future award). 
This thesis is dedicated to my parents. 



\section{Contents}

1 Introduction 1

1.1 Aim and research objectives . . . . . . . . . . . . . . . . 1

1.2 A brief history of the Bratu model . . . . . . . . . . . . . . . . . . 1

1.3 Nonstandard finite difference schemes $\ldots \ldots \ldots \ldots \ldots$. . . . . . . . . . 2

1.3 .1 Exact finite difference schemes ... . . . . . . . . . . . . . . . 3

1.3.2 Rules for constructing nonstandard finite difference schemes . . . . . . . 4

1.4 Singularly perturbed boundary value problems with boundary layers . . . . . . 6

1.5 Non-uniform grids $\ldots \ldots \ldots \ldots \ldots \ldots$

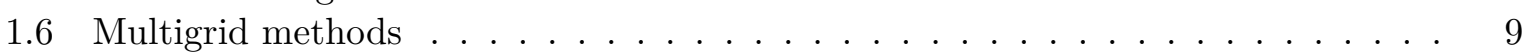

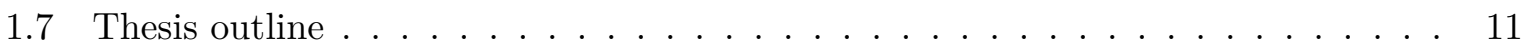

2 Nonstandard finite differences for a truncated Bratu-Picard model $\quad 15$

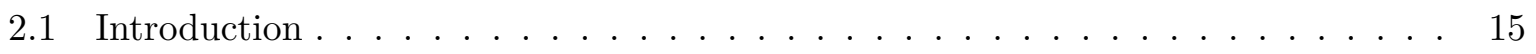

2.2 Properties of the solution $\ldots \ldots \ldots \ldots \ldots \ldots \ldots$

2.2 .1 General properties . . . . . . . . . . . . . . . . . . . 16

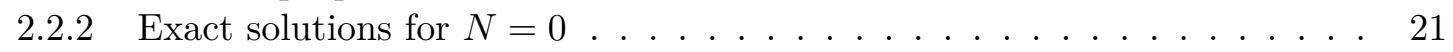

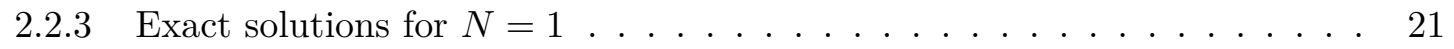

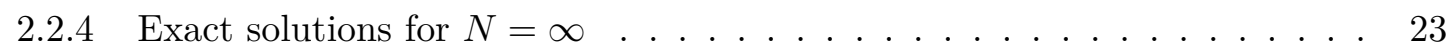

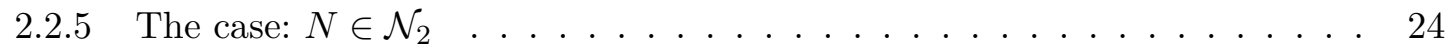

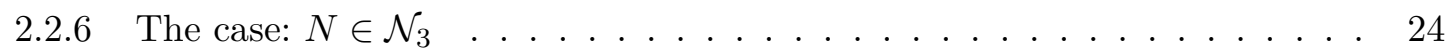

2.3 Numerical approximation . . . . . . . . . . . . . . . . . . . . 25

2.3 .1 Standard finite differences (SFD) . . . . . . . . . . . . . . . 25

2.3 .2 Nonstandard finite differences (NSFD) . . . . . . . . . . . . . . . 25

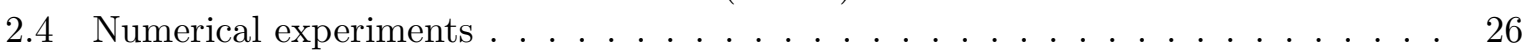

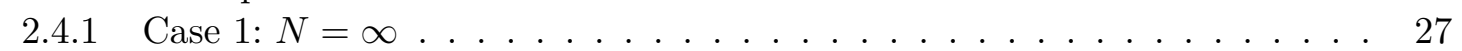

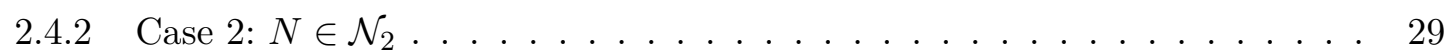

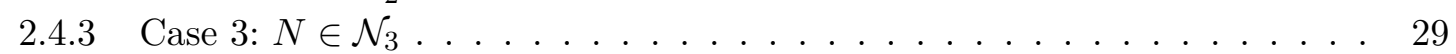

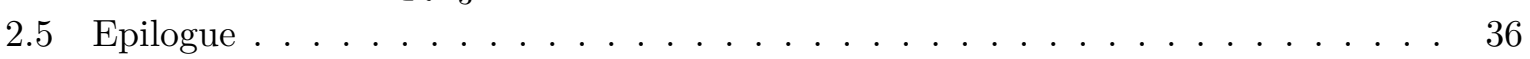

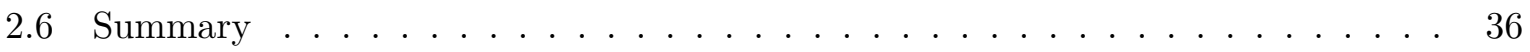

3 Higher order non-uniform grids for singularly perturbed convection-diffusionreaction problems

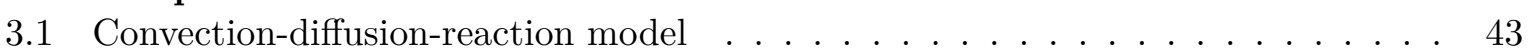

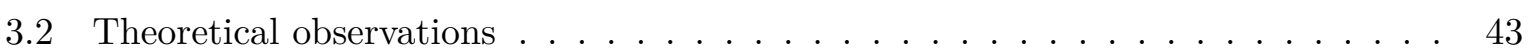

3.2 .1 Theorem 1 . . . . . . . . . . . . . . . . . . . . . . . . 43

3.2 .2 Theorem $2 \ldots \ldots \ldots \ldots \ldots \ldots \ldots$. . . . . . . . . . . . . . . . . . 44

3.3 Discretizations on uniform grids $\ldots \ldots \ldots \ldots \ldots \ldots \ldots \ldots \ldots$

3.4 Fourth-order non-uniform grid generation $\ldots \ldots \ldots \ldots \ldots$

3.4 .1 Discretizations on non-uniform grids $\ldots \ldots \ldots \ldots . \ldots . \ldots$ 
3.4 .2 Coordinate transformation . . . . . . . . . . . . . . . . . . 47

$3.4 .3 \quad$ Local truncation error analysis $\ldots \ldots \ldots$. . . . . . . . . . . . . . . . 48

3.4 .4 Numerical experiments . . . . . . . . . . . . . . . . . . . . . . . . . . 49

3.5 Sixth-order adaptive non-uniform grids . . . . . . . . . . . . . . . . 58

3.5.1 Adaptive non-uniform grid generation . . . . . . . . . . . . . . 59

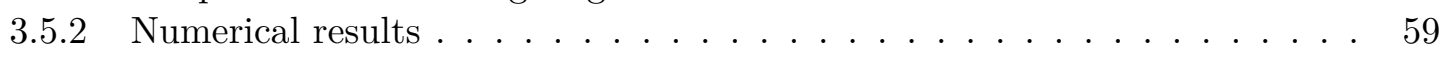

3.5.3 Numerical implementation for a nonlinear problem . . . . . . . . . . . . 62

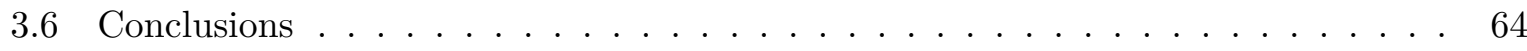

4 An efficient nonlinear multigrid scheme for two-dimensional boundary value $\begin{array}{ll}\text { problems } & 65\end{array}$

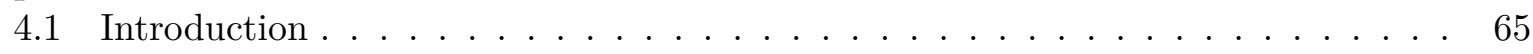

$4.2 \quad$ A description of the three numerical methods $\ldots \ldots \ldots \ldots$. . . . . . . 67

4.2.1 Method 1: a central finite difference approximation with fsolve.m . . . . . 67

4.2 .2 Multigrid methods $\ldots \ldots \ldots \ldots \ldots$. . . . . . . . . . . . . . . . . . . .

4.3 Numerical experiments . . . . . . . . . . . . . . . . . . . . . . . . 70

4.3 .1 Experiment 1: the two-dimensional Bratu problem . . . . . . . . . . . 71

4.3.2 $\quad$ Experiment 2: a quadratic approximation . . . . . . . . . . . . . . . 74

4.3.3 Experiment 3: a cubic approximation . . . . . . . . . . . . . . 76

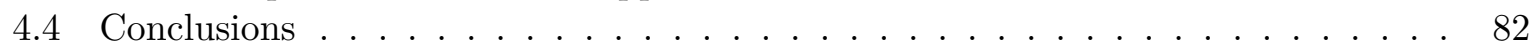

$5 \quad$ A numerical study of the higher-dimensional Gelfand-Bratu model 83

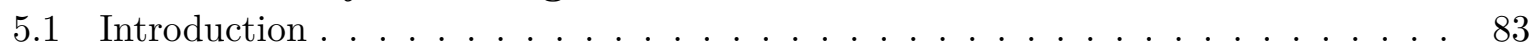

$5.2 \quad$ A boundary-value problem in $n$ space dimensions . . . . . . . . . . . . . . . 84

$5.3 \quad$ Discretization of the Laplacian in $n$ space dimensions . . . . . . . . . . . . . . . 84

5.4 The three-dimensional case $\ldots \ldots \ldots \ldots \ldots \ldots \ldots$

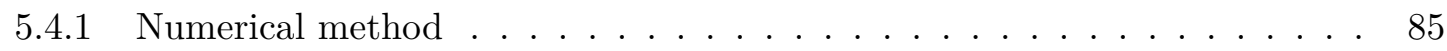

5.4 .2 Numerical results . . . . . . . . . . . . . . . . . . . . . . . . 86

5.5 The n-dimensional radial case . . . . . . . . . . . . . . . . 92

$5.5 .1 \quad$ Spherical coordinates in $n$ space dimensions . . . . . . . . . . . . . . 92

5.5.2 A boundary-value problem in $n$-dimensional spherical coordinates $\ldots . .97$

$\begin{array}{lll}5.5 .3 & \text { Theoretical results for } n \text { space dimensions in spherical coordinates }\end{array}$. . . . 97

5.5 .4 Numerical experiments . . . . . . . . . . . . . . . . . . . . . . 98

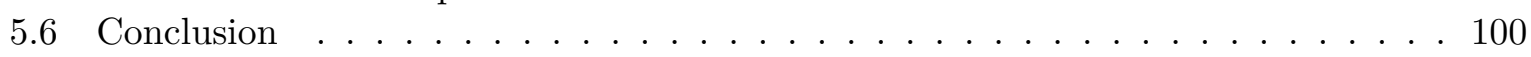

$\begin{array}{lll}6 & \text { Conclusions and outlook } & 103\end{array}$

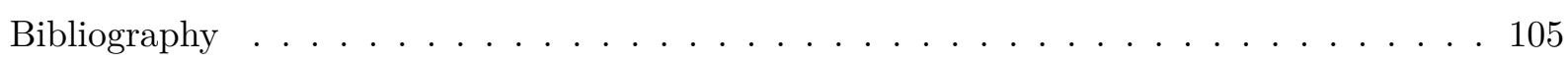

$\begin{array}{ll}\text { Samenvatting } & 115\end{array}$

Acknowledgements . . . . . . . . . . . . . . . . . . . . . 117

Curriculum Vitae . . . . . . . . . . . . . . . . . . . . . . 119 


\section{Chapter 1}

\section{Introduction}

This chapter serves as an introduction to the work presented in the thesis. It touches upon a brief history of nonlinear elliptic equations with parameter dependence such as the GelfandBratu model and singularly perturbed boundary value problems. It gives a brief sketch of the mathematical evolution of such elliptic equations and acknowledges the efforts of pioneering authors who worked in this area. The numerical methods used in current research work are described and discussed briefly. The chapter serves as a guide with research aim and objectives. Finally, the outline of this thesis is presented at the end.

\subsection{Aim and research objectives}

The aim of this thesis is to numerically investigate nonlinear elliptic boundary value problems with a parameter dependence such as the Gelfand-Bratu (GB) model and singularly perturbed convection-diffusion-reaction problems. To achieve this, different numerical strategies will be used. The main objectives of this thesis are the following:

- Numerically investigate new types of solutions (periodic and semi-periodic) and multiplicities by using a nonstandard finite difference method with different denominator functions for a truncated Bratu-Picard model.

- Develop higher-order adaptive non-uniform grids for solving singularly perturbed convectiondiffusion-reaction problems with boundary layers.

- Investigate the bifurcation behaviour of two-dimensional nonlinear boundary-value problems and detect new multiple solutions. For this, we make use of different numerical strategies, namely: finite difference approximations with a black-box Matlab-routine, a Newton-multigrid method and a nonlinear multigrid full approximation storage.

- Design an efficient nonlinear multigrid approach extended with a Krylov-method as a smoother to handle the computational difficulties for finding the new multiplicities and identify the existence of new bifurcation points for a three-dimensional GB model. Numerically investigate the bifurcation properties of the GB model for higher dimensions, as predicted from theoretical results in literature.

\subsection{A brief history of the Bratu model}

The Bratu model is an elliptic partial differential equation which appears in different models with applications such as the steady state model of the solid fuel ignition in thermal combustion 
theory [11], the Chandrasekhar model of the expansion of the universe [24] and in thermo-electrohydrodynamic models (electrospinning processes) [137] etc. Traditionally, the names Bratu [17] and, sometimes, Gelfand [42] are coupled to this model. However, in historical literature, Picard was the first one who actually introduced this model in 1898, with non-unique solutions (see [107]). The Bratu model simulates a thermal reaction process in a rigid material, where the process depends on a balance between chemically generated heat addition and heat transfer by conduction [67]. Originally, the Bratu problem appears in the study of the quasilinear parabolic problem (see [61]):

$$
\left\{\begin{array}{l}
T_{t}=\Delta T+\lambda(1-\epsilon T)^{m} \mathrm{e}^{T /(1+\epsilon T)}, \quad x \in \Omega \subset \mathbb{R}^{n}, \quad n=1,2,3 \\
T=0, \quad x \in \partial \Omega,
\end{array}\right.
$$

which is known as the solid fuel ignition model and is derived as a model for the thermal reaction process for non-deformable materials of constant density during the ignition period (see $[38,41,67])$. Here $\lambda>0$ is known as the Frank-Kamenetskii parameter, viz., $T$ is a dimensionless temperature, $\Delta$ is Laplacian, $\frac{1}{\epsilon}$ is the activation energy, $t$ is time, $m$ is the mass of a single molecule and $\Omega$ is a bounded domain (for example, a bounded container). One is interested in the question of what happens, when a combustible medium is placed in a vessel whose walls contain a fixed temperature. Nontrivial solutions of the Bratu problem appear as steady-state solutions of a solid fuel ignition model for $\epsilon \ll 1$ (see [11, 61]). If such solutions occur, then there must be a balance between cooling (due to diffusion from the boundary) and heating (because of the positive reaction term). Intuitively, one might expect that the reaction term will dominate for large values of $\lambda$ and raise the temperature to infinity (explosion), whereas for smaller $\lambda$ steady states might be possible.

In 1914, Bratu [18] found an explicit steady state solution on the unit ball $B \in \mathbb{R}^{n}$ and described the multiplicity of solutions. Later, in 1955 Frank-Kamenetskii (see [38]) was interested how to find the maximum value of the parameter $\lambda$ and its steady state solution to avoid explosion in thermal ignition processes. In 1963, Gelfand [42] built on Frank-Kamenetskii's work to prove the existence of values of $\lambda$ for which the fuel ignition model has infinitely many nontrivial solutions. An exact multiplicity and an S-shaped bifurcation curve for some semi-linear elliptic problems from combustion theory are presented in [32, 135]. Jacobsen and Schmitt provided an excellent summary of the significance and history of the classical Gelfand-Bratu (GB) problem in detail (see [61]). In the past two decades, several numerical techniques have been presented to compute the numerical solutions for the GB problem on different domains and dimensions. Most of the numerical methods lose their accuracy significantly, when $\lambda$ approaches the critical value $\lambda_{c}$. Only a few numerical schemes have been developed to obtain convergence to both solutions (lower and upper) for different values of $\lambda$. Nowadays, it is still very challenging to compute accurate numerical solutions, especially finding the multiplicities of the GB model in higher dimensions for $\lambda \in\left(0, \lambda_{c}\right]$ on different types of domains.

\subsection{Nonstandard finite difference schemes}

The majority of differential equation models (both linear and nonlinear), which correspond to dynamical systems stemming from engineering and natural sciences, can't be solved exactly in terms of elementary functions. As a consequence, a variety of numerical methods has been constructed to find an approximate solution. One of the traditional techniques in this area, is the finite difference method [78, 11, 52, 128, 73]. Unfortunately, numerical instabilities may arise in these finite difference approximations. Normally, numerical instabilities can be recognized as 
the solutions to the discrete finite difference equations that do not correspond to any solution of the original differential equations. To eliminate or minimize such difficulties, Mickens [85, 86 introduced the concept of nonstandard finite difference schemes (NSFD) in 1989 for some classes of differential equations. Recently, NSFD discrete models have been constructed for singular boundary value problems [20], reaction-diffusion models [25, 90, 88], the dynamics of HIV transmission [45], modified linear transport equations [87, 91] and many more applications. To understand the general rules how to construct an NSFD method, we first present the concept of an exact finite difference scheme.

\subsubsection{Exact finite difference schemes}

An exact finite difference (EFD) scheme is one for which the solution of the difference equation has exactly the same general solution as the associated differential equation at the grid points.

The construction of exact schemes can be illustrated by considering the first order differential equation

$$
\frac{d u}{d t}=f(u, \lambda, t), \quad u\left(t^{0}\right)=u^{0}
$$

where $\lambda$ is a system parameter and $f$ is such that equation (1.1) has a unique solution. This solution can be written as

$$
u(t)=\phi\left(\lambda, u^{0}, t^{0}, t\right)
$$

The exact finite difference scheme of equation (1.1) is then given by

$$
u^{n+1}=\phi\left(\lambda, u^{n}, t^{n}, t^{n+1}\right) .
$$

where $t^{n}=n \Delta t, u^{n} \approx u\left(t^{n}\right)$ and the function $\phi$ is that of equation (1.2). To explain an exact finite difference scheme, we present two dynamical systems as examples; one ordinary and one partial differential equation.

Example 1.3.1:

Consider the decay equation

$$
\frac{d u}{d t}=\lambda u, \quad u\left(t^{0}\right)=u^{0}, \lambda>0 .
$$

Then, the exact solution reads

$$
u(t)=u^{0} \mathrm{e}^{\lambda\left(t-t^{0}\right)} .
$$

From this expression, an exact finite difference scheme can be derived of the form:

$$
u^{n+1}=u^{n} \mathrm{e}^{-\lambda \Delta t} .
$$

After some rearranging, this expression can be rewritten as:

$$
\frac{u^{n+1}-u^{n}}{\left(\frac{1-\mathrm{e}^{-\lambda \Delta t}}{\lambda}\right)}=-\lambda u^{n} .
$$

Comparing this with the standard Euler-forward (EF) scheme for differential equation (1.3), shows one important difference in the denominator on the left hand side:

$$
\frac{u^{n+1}-u^{n}}{\Delta t}=-\lambda u^{n} .
$$


It is known that EF is numerically unstable for $\Delta t>\frac{-2}{\lambda}$, whereas the EFD method is unconditionally stable for all $\Delta t>0$.

Example 1.3.2:

Consider next, the one-dimensional advection equation with a nonlinear reaction term [90]:

$$
u_{t}+u_{x}=u(1-u), \quad u(x, 0)=f(x),
$$

which has the exact solution

$$
u(x, t)=\frac{f(x-t)}{\mathrm{e}^{-t}+\left(1-\mathrm{e}^{-t}\right) f(x-t)} .
$$

The EFD scheme for PDE (1.4) in [90] reads:

$$
\frac{u_{j}^{n+1}-u_{j}^{n}}{\phi(\Delta t)}+\frac{u_{j}^{n}-u_{j-1}^{n}}{\phi(\Delta x)}=u_{j-1}^{n}\left(1-u_{j}^{n+1}\right),
$$

with $\phi(\Delta t)=\mathrm{e}^{\Delta t}-1=\Delta t+\mathcal{O}\left((\Delta t)^{2}\right)$ and $\phi(\Delta x)=\Delta x+\mathcal{O}\left((\Delta x)^{2}\right)$. This expression is linear in $u_{j}^{n+1}$, from which we obtain the explicit EFD scheme:

$$
u_{j}^{n+1}=\frac{u_{j-1}^{n}}{1+\left(\mathrm{e}^{\Delta t}-1\right) u_{j-1}^{n}} .
$$

Based on these two (and many other) examples, rules for constructing nonstandard finite difference (NSFD) schemes for differential equations can be formulated, as we will see in the next section.

\subsubsection{Rules for constructing nonstandard finite difference schemes}

A nonstandard finite difference scheme is any discrete representation of a system of differential equations that is constructed according to the following rules (see [90]):

- Rule 1. The order of the discrete derivatives should be equal to the orders of the corresponding derivatives of the differential equations. This rule demonstrates that spurious solutions may exist, if the order of the discrete derivatives is larger than that of the derivatives that appear in the differential equations. The following example shows the consequences, if Rule 1 is violated.

Consider the decay differential equation:

$$
\frac{d u}{d t}=-u \text {. }
$$

A central finite difference approximation for ODE (1.5) gives:

$$
u^{n+1}+2 \Delta t u^{n}-u^{n-1}=0 .
$$

It has the general solution [89]:

$$
u^{n}=C_{1}\left(r_{+}\right)^{n}+C_{2}\left(r_{-}\right)^{n}, \quad \text { with } \quad r_{ \pm}=-\Delta t \pm \sqrt{1+(\Delta t)^{2}},
$$

where $C_{1}$ and $C_{2}$ are arbitrary constants. The $r_{ \pm}$are functions of $\Delta t$ and the following result holds for large $\Delta t$ : 


$$
\left\{\begin{array}{l}
r_{+}(\Delta t)=\frac{1}{2 \Delta t}+\mathcal{O}\left(\frac{1}{(\Delta t)^{3}}\right) \\
r_{-}(\Delta t)=2 \Delta t+\mathcal{O}\left(\frac{1}{\Delta t}\right)
\end{array}\right.
$$

From this follows, that the second term of the general solution (1.6), exponentially increases with $n$, whereas the first term decreases monotonically to zero. Since this behaviour holds for any step size $\Delta t$, we see that a second-order central finite difference approximation for a first-order derivative may produce numerical instabilities.

- Rule 2. Discrete derivatives could have nontrivial denominator functions $\phi$. For example, the first discrete derivative has the form

$$
\left.\frac{d u}{d t}\right|_{t^{n}} \approx \frac{u^{n+1}-\psi u^{n}}{\phi},
$$

where $\psi$ and $\phi$ may depend on the step-size $\Delta t$ and satisfy the conditions

$$
\psi=1+\mathcal{O}(\Delta t), \quad \phi=\Delta t+\mathcal{O}\left((\Delta t)^{2}\right) .
$$

- Rule 3. Nonlinear terms in the differential equation could be modeled by nonlocal discrete representations. For example, at the time grid points $t^{n}, u^{2}$ could be approximated, not by $\left(u^{n}\right)^{2}$, but by $\left(u^{n}\right)^{2} \rightarrow u^{n+1} u^{n}$.

- Rule 4. Special conditions for differential equations must hold also for a finite difference scheme. For example, many dynamical systems hold a condition of positivity for the dependent variables. If the numerical scheme violates this condition, then numerical instabilities are likely to occur [92].

Note that, in general, nonstandard schemes do not correspond to exact schemes, but can be helpful for the construction of schemes to eliminate elementary numerical instabilities. In the limit $\Delta t \rightarrow 0$ nonstandard and standard FD schemes are identical, but for finite small values of $\Delta t$ they are, obviously, not.

Example 1.3.3:

Consider the dynamical system [89, 90]:

$$
\frac{d u}{d t}=u^{2}(1-u)
$$

The discrete derivative reads:

$$
\left.\frac{d u}{d t}\right|_{t^{n}} \approx \frac{u^{n+1}-u^{n}}{\phi(\Delta t)}
$$

where the denominator function is: $\phi(\Delta t)=1-\mathrm{e}^{-\Delta t}$ such that $0<\phi(\Delta t)<1, \Delta t>0$ and $u(t)$ represents a nonnegative quantity. The following condition must, therefore, also hold for the numerical approximations:

$$
u^{n} \geq 0 \Rightarrow u^{n+1} \geq 0, \quad \forall n \geq 0 .
$$

It is possible to deduce, that, if the nonlinear terms $u^{2}$ and $u^{3}$ in (1.7) are replaced by nonlocal discrete representations:

$$
\left(u^{n}\right)^{2} \rightarrow 2\left(u^{n}\right)^{2}-u^{n+1} u^{n}, \quad\left(u^{n}\right)^{3} \rightarrow u^{n+1}\left(u^{n}\right)^{2},
$$


then the explicit nonstandard scheme for $u^{n+1}$ of equation (1.7) becomes:

$$
u^{n+1}=\frac{\left(1+2 \phi u^{n}\right) u^{n}}{1+\phi\left[u^{n}+\left(u^{n}\right)^{2}\right]} .
$$

It can be shown that the $u^{n}$ from $(1.9)$ satisfy condition $(1.8)$, which can not at all be guaranteed by a standard finite difference scheme.

\section{Example1.3.4:}

Consider the reaction-diffusion PDE:

$$
\left\{\begin{array}{l}
u_{t}=u_{x x}+u(u-1), \\
u(x, 0)=x(2 \pi-x) / \pi^{2},
\end{array}\right.
$$

which may have the following nonstandard FD scheme (for more details see [88]):

$$
\frac{u_{j}^{n+1}-u_{j}^{n}}{\phi(\Delta t)}=\frac{u_{j+1}^{n}-2 u_{j}^{n}+u_{j-1}^{n}}{(\Delta x)^{2}}+\left(\frac{u_{j-1}^{n}+u_{j}^{n}+u_{j+1}^{n}}{3}\right)\left(u_{j}^{n+1}-1\right),
$$

with $\phi(\Delta t)=1-\mathrm{e}^{\Delta t}$. The numerical solution of PDE (1.10) can be calculated using a standard FD scheme as:

$$
\frac{u_{j}^{n+1}-u_{j}^{n}}{\Delta t}=\frac{u_{j+1}^{n}-2 u_{j}^{n}+u_{j-1}^{n}}{(\Delta x)^{2}}+u_{j}^{n}\left(u_{j}^{n}-1\right),
$$

or using the nonstandard FD scheme (1.11) for $\Delta t=0.1$ and $\Delta x=0.25$. Figure 1.1 shows that the nonstandard scheme remains stable and the standard scheme not. Nonstandard finite
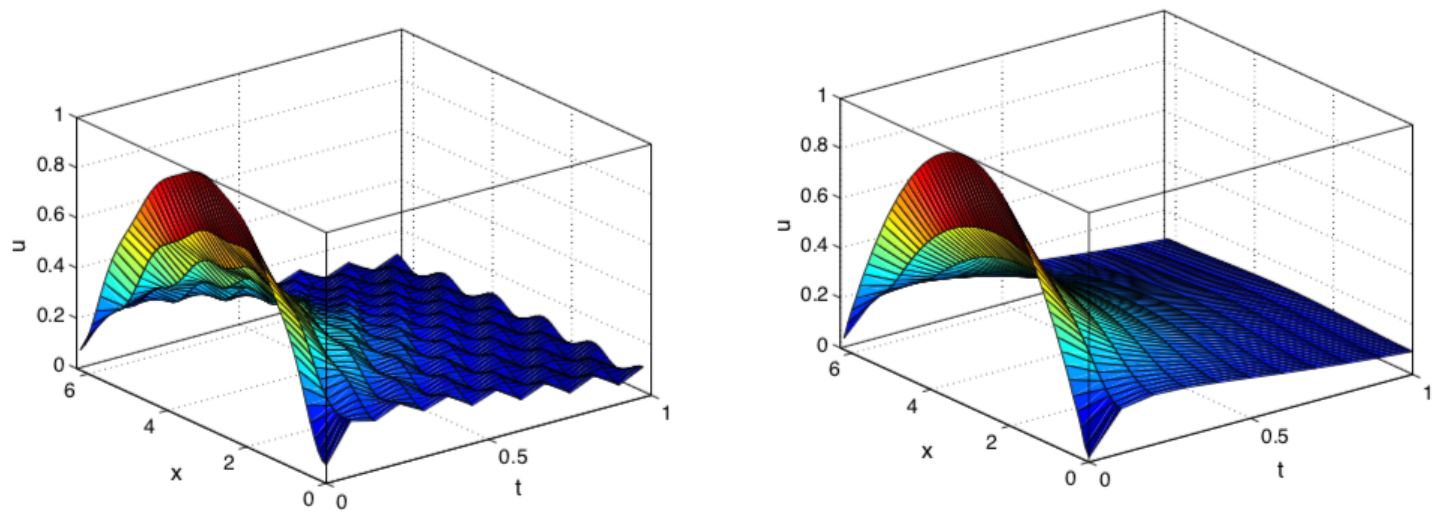

Figure 1.1: Numerical solutions using a standard FD scheme (1.12) and a nonstandard FD scheme (1.11). The solution on the right remains stable (nonstandard), whereas the solution on the left possesses numerical instabilities (standard).

difference schemes will be applied to nonlinear boundary-value problems, in Chapter 2 of this thesis.

\subsection{Singularly perturbed boundary value problems with bound- ary layers}

It is well-known that many phenomena in fluid dynamics, biology, engineering, physics and many more can be described by boundary value problems (BVPs) associated with different types 
of partial differential equations or dynamical systems [114, 2, 111]. In a perturbed mathematical model, in which the highest derivative is associated with a small parameter $\epsilon$, the perturbation is called singular. If the solution of the perturbation problem exhibits fast variations within small regions, then the solution has a boundary layer. We give a simple example to show a typical boundary layer in a singularly perturbed BVP.

Example 1.4.1:

Consider the one-dimensional singularly perturbed BVP (see also [75]):

$$
\left\{\begin{array}{l}
\epsilon u^{\prime \prime}+2 u^{\prime}+2 u=0, \quad 0<x<1, \quad 0<\epsilon \ll 1, \\
u(0)=0, u(1)=1,
\end{array}\right.
$$

which has the exact solution:

$$
u(x)=\frac{\mathrm{e}^{r_{1} x}-\mathrm{e}^{r_{2} x}}{\mathrm{e}^{r_{1}}-\mathrm{e}^{r_{2}}}, \text { with } r_{1,2}=(-1 \pm \sqrt{1-2 \epsilon}) / \epsilon .
$$

Figure 1.2 shows the boundary layer at $x=0$ of this model for decreasing values of $\epsilon$.

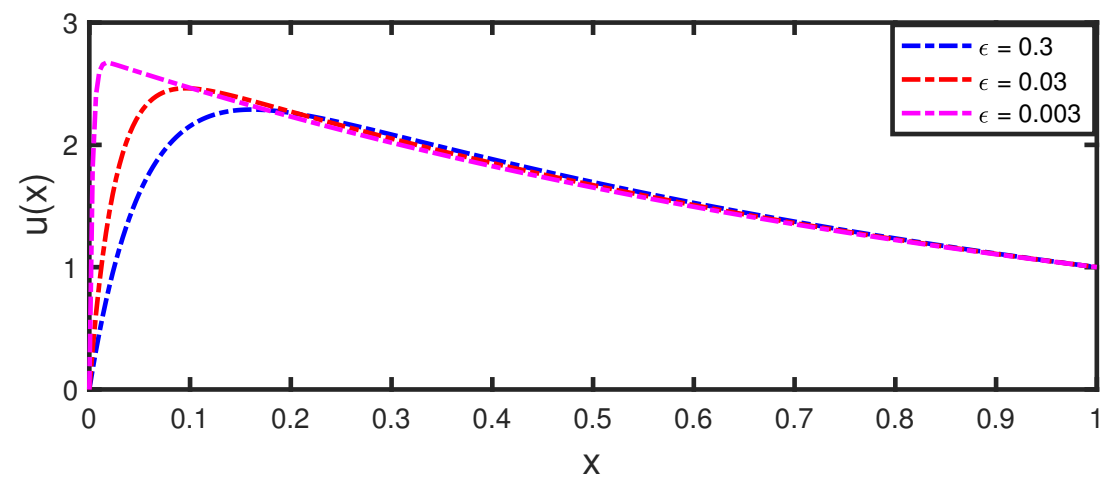

Figure 1.2: Numerical solution of the singularly perturbed BVP (1.13) for small values of the parameter $\epsilon>0$.

The term boundary layer is a well known concept in the research of singular perturbation problems. Singular perturbations have their birth in boundary layer theory in fluid dynamics due to Prandtl in 1904 [108]. The notion of singular perturbations was first introduced by Friedrichs and Wasow [39]. Later on, singular perturbations for ordinary differential equations were further developed by Tikhonov [130] and Vasil'eva [133]. A solid theoretical basis boundary layer theory was elaborated by Eckhaus [34, 33]. An excellent historical survey on singular perturbations can be found in the book by O'Malley [103]. Applications and methods of singular perturbations in boundary layer problems were presented in detail by Verhulst [135].

Nowadays, nonlinear boundary layer problems are addressed with specialized numerical techniques. A large number of numerical techniques have been proposed by various authors for such models. Finite difference approximations for boundary layer problems on non-uniform grids have been discussed in [134], [125], [5], [135], [109]. Special piecewise uniform grids have been introduced by Shishkin [93], in which relatively simple structured grids can be used for the numerical approximation of boundary layer problems. A truncation error analysis introduced by the use of non-uniform grids and stretched coordinates has been reported in [30], [54], [72], [136], [79], [29], [101]. Generally, numerical methods for boundary layer problems on non-uniform grids are 
more accurate than for the uniform grid case, but with the same order of convergence. In this thesis not only more accurate, but also higher-order non-uniform grids ('supra-convergence') for singularly perturbed boundary-value problems will be treated (details can be found in Chapter 3).

\subsection{Non-uniform grids}

Finite difference schemes on uniform grids are relatively simple methods to obtain the numerical solution of boundary value problems [106, 77, 129, 122]. However, they may not be accurate and efficient for problems with boundary layers or moving fronts (travelling waves), because of the unphysical oscillations that appear in numerical solutions [6, 4]. If the number of points is not sufficiently high to resolve the boundary layer, the numerical solution is likely to have huge errors. The use of a large number of grid points to resolve this problem makes the total computation time unacceptably large. To eliminate the numerical oscillations in the solutions, one needs a very fine grid in the layer regions. This situation may be improved, either by using non-uniform grids (in a prescribed way) or by an adaptive grid (generated by an additional equation) [31, 79, 12, 22]. Numerical methods based on non-uniform grids concentrate the grid points in steep regions of solution (boundary layer), whereas in smoother regions of the solution fewer grid points can be used [5, 134, 119] (see Figure 1.3).
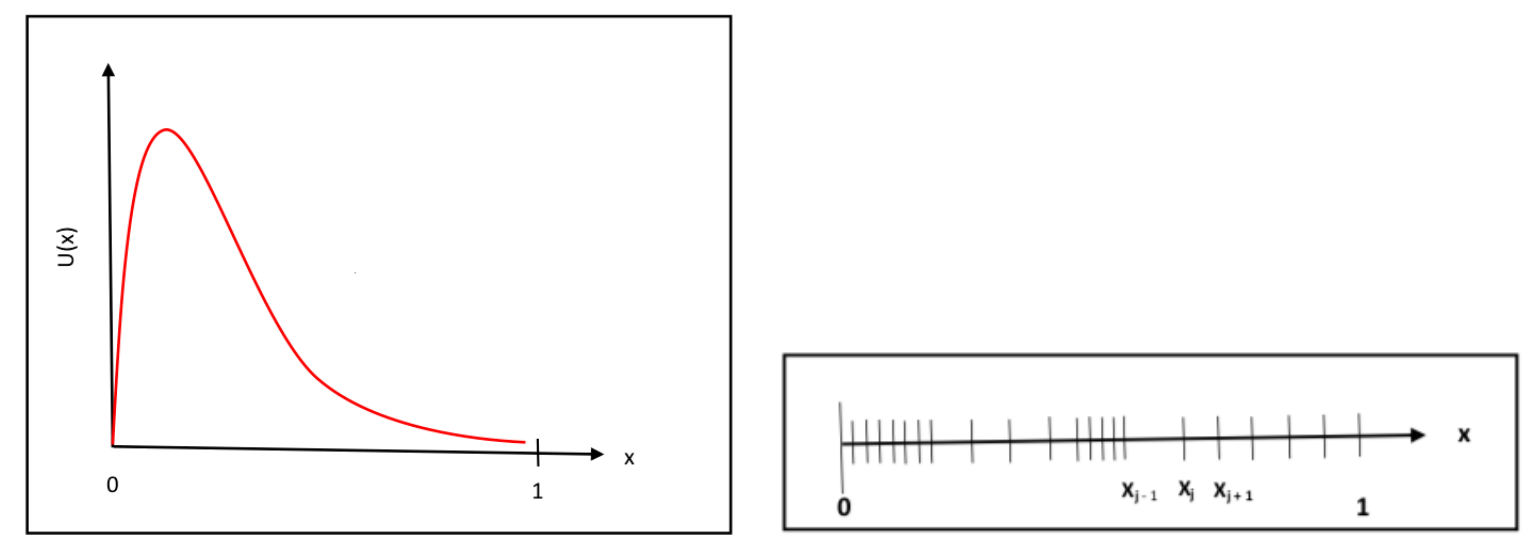

Figure 1.3: Solution of a boundary-value model with boundary layer at $x=0$ (left) and an example of a non-uniform grid distribution to resolve the boundary layer and the internal steep gradient in the solution (right).

Approximation of derivatives:

For the approximation of the first derivative, consider a non-uniform grid (see Figure 1.3) with

$$
\Delta x_{j}:=x_{j}-x_{j-1}, \quad \Delta x_{j+1}:=x_{j+1}-x_{j}, \quad r_{j}:=\frac{\Delta x_{j+1}}{\Delta x_{j}} .
$$

Consider a three-point representation of the first derivative:

$$
\left.\frac{d u}{d x}\right|_{x_{j}} \approx a u_{j-1}+b u_{j}+c u_{j+1},
$$

with $u_{j-1} \approx u\left(x_{j}-\Delta x_{j}\right), u_{j} \approx u\left(x_{j}\right), u_{j+1} \approx u\left(x_{j}+\Delta x_{j+1}\right)$ and the coefficients can be determined using Taylor expansions around $x=x_{j}$ (see details in [118, 141]):

$$
a=-\frac{\Delta x_{j+1}}{\Delta x_{j}\left(\Delta x_{j}+\Delta x_{j+1}\right)}, \quad b=\frac{\Delta x_{j+1}-\Delta x_{j}}{\Delta x_{j} \Delta x_{j+1}}, \quad c=\frac{\Delta x_{j}}{\Delta x_{j+1}\left(\Delta x_{j}+\Delta x_{j+1}\right)} .
$$


Then, the first derivative on nonuniform grids becomes:

$$
\underbrace{\frac{1}{\Delta x_{j}+\Delta x_{j+1}}\left[\frac{1}{r_{j}}\left(u_{j+1}-u_{j}\right)+r_{j}\left(u_{j}-u_{j-1}\right)\right]}_{\text {approximation }}=\underbrace{\left.\frac{d u}{d x}\right|_{x_{j}}}_{\text {exact }}-\underbrace{\left.\frac{1}{6} \Delta x_{j} \Delta x_{j+1} \frac{d^{3} u}{d x^{3}}\right|_{x_{j}}+\ldots}_{\text {error }}
$$

Similarly, a three-point second derivative can be represented as:

$$
\left.\frac{d^{2} u}{d x^{2}}\right|_{x_{j}} \approx \tilde{a} u_{j-1}+\tilde{b} u_{j}+\tilde{c} u_{j+1},
$$

and we can compute the coefficients using Taylor expansions

$$
\tilde{a}=\frac{2}{\Delta x_{j}\left(\Delta x_{j}+\Delta x_{j+1}\right)}, \quad \tilde{b}=\frac{2}{\Delta x_{j} \Delta x_{j+1}}, \quad \tilde{c}=\frac{2}{\Delta x_{j+1}\left(\Delta x_{j}+\Delta x_{j+1}\right)},
$$

which gives a relation between the approximation, the exact value and the error for the second derivative on nonuniform grids:

$$
\underbrace{\frac{2}{\Delta x_{j}\left(\Delta x_{j}+\Delta x_{j+1}\right)}\left[\frac{1}{r_{j}}\left(u_{j+1}-u_{j}\right)+r_{j}\left(u_{j}-u_{j-1}\right)\right]}_{\text {approximation }}=\underbrace{\left.\frac{d^{2} u}{d x^{2}}\right|_{x_{j}}}_{\text {exact }}-\underbrace{\left.\frac{1}{3} \Delta x_{j}\left(r_{j}-1\right) \frac{d^{3} u}{d x^{3}}\right|_{x_{j}}+\ldots}_{\text {error }}
$$

In this way, one can construct a finite difference scheme to approximate derivatives on a nonuniform grid. Many numerical techniques have been proposed for boundary layer problems. Some of these approaches on non-uniform grids are more accurate than for the uniform case. The following example compares numerical solutions computed by using uniform and non-uniform grids for a typical boundary layer problem.

Example 1.5.1:

Consider a nonlinear initial-boundary-value problem with a boundary layer at $x=1$ for $t \rightarrow \infty$ :

$$
\left\{\begin{array}{l}
u_{t}=\epsilon u_{x x}+2 u_{x}+\mathrm{e}^{u} \\
u(0, t)=u(1, t)=0, u(x, 0)=\sin (\pi x) .
\end{array}\right.
$$

The numerical solutions are computed using a finite difference method on uniform and nonuniform grids, for $\epsilon=0.001$ and 151 grid points (see Figure 1.4). The results clearly show the benefits of non-uniform grids for models with steep layers. Non-uniform grids will be discussed and applied to several boundary-value problems, which exhibit boundary layer(s), in Chapter 3 of this thesis.

\subsection{Multigrid methods}

Multigrid (MG) algorithms are useful for solving systems of equations that are linear or nonlinear, such as elliptic and parabolic partial differential equations [16, 46, 15, 132], bifurcation models [95, 127, 100], integral equations and eigenvalue problems. These algorithms have been applied to lattice field computations [44], quantum electrodynamics [65], chromodynamics simulations [81] and many other applications.

In the 60's of the last century, Fedorenko [36, 37] formulated the first MG algorithm based on a standard five-point finite difference discretization for the solution of the Poisson equation on a square. Since then, his idea has been extended to general elliptic BVPs with variable coefficients 

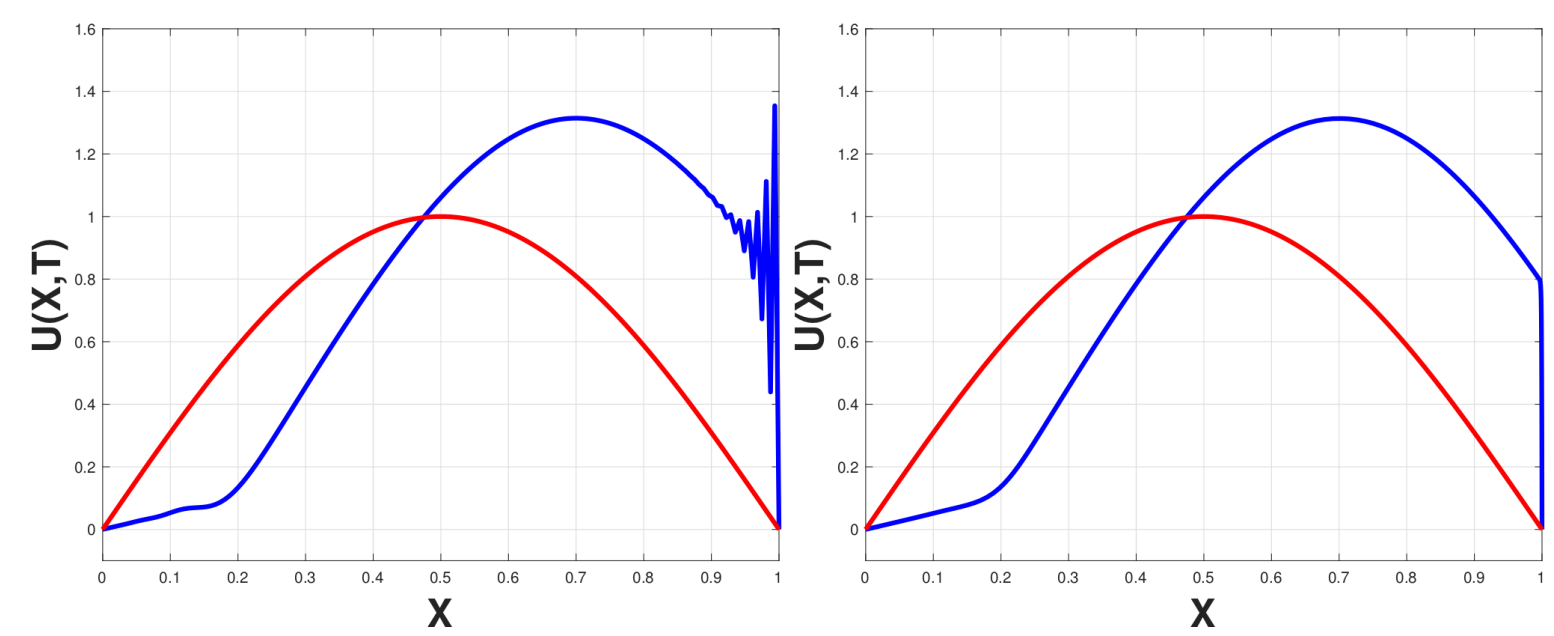

Figure 1.4: Numerical solutions of model (1.14): severe oscillations appear using a uniform grid (left), whereas no oscillations are produced on the adaptive non-uniform grid (right) for $\epsilon=0.001$ and 151 grid points at $t=0.1$. The red curve shows the initial condition. The adaptive grid is based on an equidistribution principle with smoothing [31, 22].

(see [6]). Later in the 70's, main principles and practical results of MG methods were reported by Brandt [15]. The work of Brandt is regarded as a landmark in the field of the development of MG and has set solid foundations for further research. Hackbusch [50] deployed a mathematical foundation for the theory of MG methods. Prominent research in this area includes the books and articles by Stüben, Wesseling, McCormick, Trottenberg, Oosterlee, Schüller [123, 124, 140, 142, 104, 82, 131].

MG methods have a recursive structure. In the MG approach, the convergence rate is independent of the grid size, which makes MG suitable for the efficient iterative solution of very large discretized systems of equations. An MG scheme is based on a sequence of grids obtained by successive coarsening. When considering only two grids, $h$ and $2 h$ are used to simplify notation for fine and coarse grids (see Figure 1.5). A classical multigrid approach begins with a MG cycle
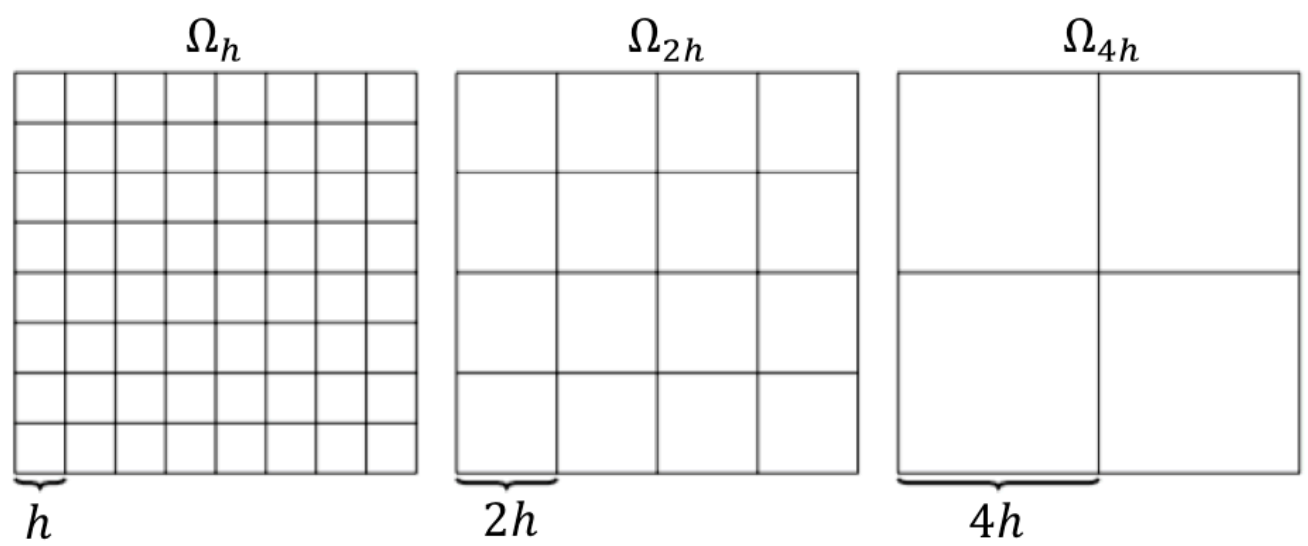

Figure 1.5: Sequence of grids with spacing $h$ (fine grid), $2 h, 4 h$ (coarsest grid) of the set of grid points $\Omega_{h}, \Omega_{2 h}$ and $\Omega_{4 h}$, respectively. 
process for solving the system of equations $A_{h} u_{h}=f_{h}$, where $u_{h}$ is the approximate solution. In this process, first, an iterative relaxation is applied to approximate the solution on the fine grid, whose effect is to smooth the error. The residual belonging to the fine grid approximation of the solution is transferred by a restriction operator to a coarse grid. Since the problem on a coarse grid is similar to the fine grid problem, the same iterative relaxation may be applied to eliminate the corresponding error. This process is recursively applied until the coarsest grid is reached, where the direct solution of the problem can be obtained easily. Then, the coarsest grid correction is used in which the smooth error is determined. This error is interpolated to the fine grid and used to correct the fine grid approximation. This idea is recursively followed until a reliable convergence is obtained. A flow diagram (see Figure 1.6) explains typical MG-cycles in more detail. The coarse grid defect equation in MG method can be solved approximately by recursively coarsening the grid. The iterations during the recursive process on the coarse grid are usually characterized by the cycles index $\gamma$. For $\gamma=1$ and $\gamma=2$, we have a $V$-cycle and a $W$-cycle, respectively, see Figure 1.7.

Many problems of physical interest are nonlinear and for such problems a modification of the MG strategy provides new powerful algorithms. In general, there are two main MG approaches for solving nonlinear problems. The first is to apply a global linearization scheme, such as Newton's method. After having linearized the PDE problem, the MG method can be used for solving the resulting Jacobian system in each iteration. Such a scheme is called the Newton multigrid method in which Newton's method is used in an outer iteration and linear MG solves in the inner iterations. The second approach is to make use of multigrid directly in the nonlinear problem by employing the full approximation storage (FAS). A nonlinear iteration is applied to smooth the error in FAS . The full equation is solved on the coarse grid, after which the coarse-grid error is extracted from the solution. This correction is then interpolated and applied to the fine grid approximation. For FAS to be effective in a multigrid method, it must be combined with a relaxation scheme to ensure that the error is smooth before extracting it from the coarse grid. Hence, we require a relaxation method for nonlinear systems. Some of the more frequently used relaxation methods are nonlinear Gauss-Seidel and Weighted Jacobi methods [105]. The MG algorithms can be modified by adding and combining some Krylov methods as smoothers to enhance the multigrid efficiency. Washio and Oosterlee [138] presented a nonlinear Krylov acceleration method as the outer iteration with a MG preconditioner. Two Krylov methods as a smoother to multigrid based methods for elliptic problems were employed by Mohsen [97], in which the MINRES method was proposed as a smoother to improve the convergence [8]. Here, in this thesis, we restrict our attention to the application of MG methods in nonlinear elliptic partial differential equations. We will see that MG schemes are efficient and fast elliptic equation solvers for these types of applications. In Chapter 4 and Chapter 5, nonlinear MG methods will be described in more detail, and numerical experiments will demonstrate their efficiency.

\subsection{Thesis outline}

Chapter 2 is based on the article [148]. In this chapter, theoretical and numerical properties of a nonlinear boundary-value problem, which is strongly related to the well-known GelfandBratu (GB) model with a bifurcation parameter $\lambda$, are presented. When approximating the nonlinear term in the model via a Taylor expansion, new types of solutions and multiplicities are found, depending on the final index $N$ in the expansion. The number of solutions may vary from $0,1,2$ to $\infty$. In the latter case of infinitely many solutions, both periodic and semi-periodic solutions are obtained. Numerical experiments using a non-standard finite-difference (NSFD) approximation illustrate all these aspects. The difference in accuracy is shown for different denominator functions in NSFD when applied to the GB model. A full classification is given of 


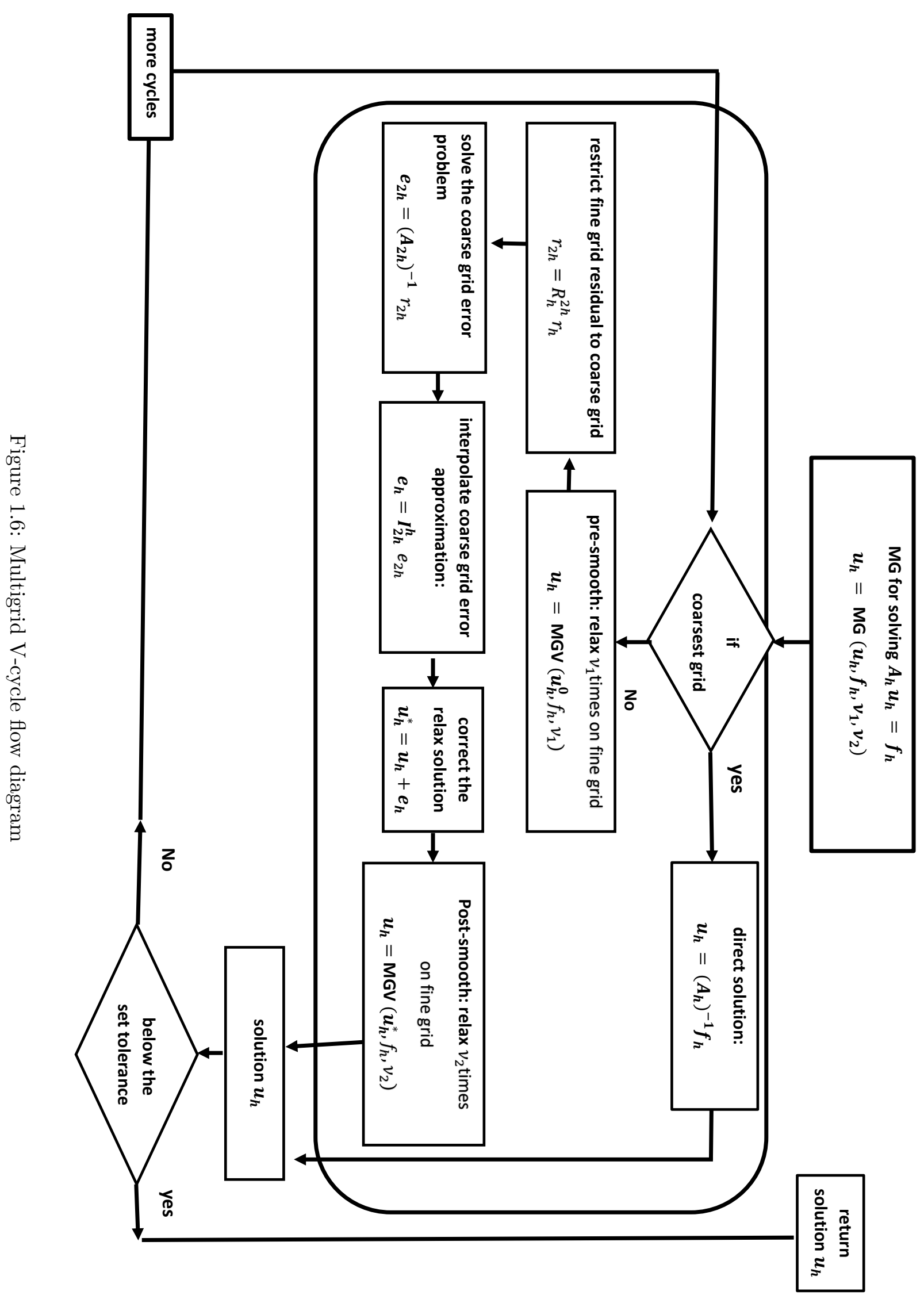




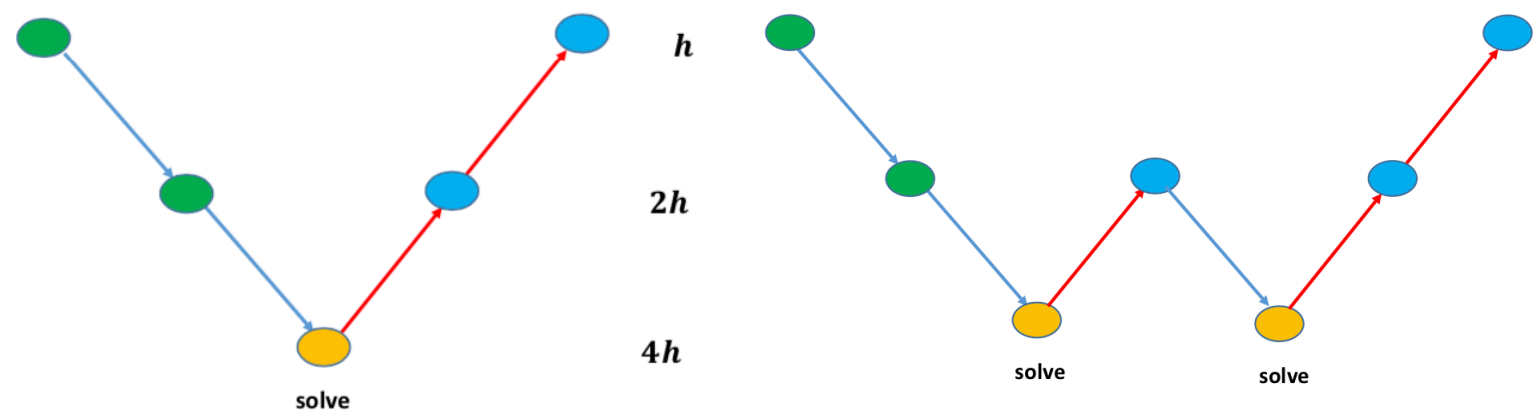

Figure 1.7: A V-cycle (left) and a W-cycle (right ) for a 3-grid multigrid method. The green and blue circles show the pre-smoothing and post-smoothing, respectively. The orange circles represent the solution of the error problem on the coarse grid (see Figure 1.6). The blue and red arrows denote the restriction and interpolation, respectively.

all possible types of solutions, depending on the parameters $N$ and $\lambda$.

In chapter 3, a higher-order non-uniform grid strategy is developed for solving singularly perturbed convection-diffusion-reaction problems with boundary layers. A new non-uniform grid finite-difference method (FDM) based on a coordinate transformation is adopted to establish higher-order accuracy. To achieve this, the truncation error of the discretized system is utilized to obtain a fourth-order non-uniform grid transformation. Considering a three-point central finite-difference scheme, not only fourth-order but even sixth-order approximations (which is the maximum order that can be obtained) are developed by a suitable choice of the underlying non-uniform grids. Further, an adaptive non-uniform grid method based on an equidistribution principle is used to demonstrate the sixth-order of convergence. Unlike several other adaptive numerical methods, the proposed strategy uses no pre-knowledge of the location and the width of the layers. Numerical experiments for various test problems are presented to verify the theoretical aspects. Numerical results illustrate that slightly different choices of the grid distributions lead to a substantial degradation of the accuracy.

In chapter 4, a two-dimensional nonlinear boundary value problem which is strongly related to the well-known Gelfand-Bratu model is solved numerically. The numerical results are obtained by employing three different numerical strategies namely: a finite difference based method, a Newton multigrid method and a nonlinear multigrid full approximation storage (FAS) method. We are able to handle the difficulty of unstable convergence behaviour by using the MINRES method as a relaxation smoother in the multigrid approach with an appropriate sinusoidal approximation as an initial guess. A comparison, in terms of convergence, accuracy and efficiency among the three numerical methods demonstrates an improvement for the values of $\lambda \in\left(0, \lambda_{c}\right]$. Numerical results illustrate the performance of the proposed numerical methods wherein the FAS-MG method is shown to be the most efficient. Further, we present the numerical bifurcation behaviour for two-dimensional Gelfand-Bratu models and find new multiplicity of solutions in the case of a quadratic and cubic approximation of the nonlinear exponential term. Numerical experiments confirm the convergence of the solutions for different values of $\lambda$ and prove the effectiveness of the nonlinear FAS-MG scheme.

In chapter 5, a higher-dimensional Gelfand-Bratu problem is discussed numerically. For the three-dimensional case, an accurate and efficient nonlinear multigrid approach is presented and we investigate multiplicities depending on the bifurcation parameter $\lambda$. A nonlinear multigrid approach, the full approximation storage (FAS) scheme, extended with a Krylov method as a 
smoother is adopted to handle the computational difficulties for obtaining the upper branches of the solutions. Furthermore, the numerical bifurcation behaviour of the Gelfand-Bratu problem in $3 \mathrm{D}$ is examined and identifies the existence of two new bifurcation points. Experiments illustrate the convergence of the numerical solutions and demonstrate the effectiveness of the proposed numerical strategy for all parameter values $\lambda \in\left(0, \lambda_{c}\right]$. For even higher dimensions, we transform the Gelfand-Bratu problem, using $n$-dimensional spherical coordinates, to a nonlinear ordinary differential equation (ODE). The numerical solutions of this nonlinear ODE are computed by a shooting method for a range of values of the dimension parameter $n$. Numerical experiments show the existence of several types of solutions for different values of $n$ and $\lambda$. These results confirm the bifurcation behaviour of the higher-dimensional Gelfand-Bratu problem as predicted from theoretical results in literature.

Finally, some main conclusions of this thesis are presented in the Summary chapter. 


\section{Chapter 2}

\section{Nonstandard finite differences for a truncated Bratu-Picard model}

\subsection{Introduction}

In this chapter, we consider smooth (continuous) solutions of the following truncated BratuPicard (tBP) model:

$$
\left\{\begin{array}{l}
u^{\prime \prime}(x)+\lambda \sum_{n=0}^{N} \frac{[u(x)]^{n}}{n !}=0, \quad x \in[0,1], \quad \lambda \in \mathbb{R}, \quad N \in \mathbb{N} \cup\{0\} \cup\{\infty\}, \\
u(0)=u(1)=0
\end{array}\right.
$$

For $N=\infty$ and $\lambda \geq 0$, this yields the classical Gelfand-Bratu model [107, 17, 18, 42] for which an exact solution is known, see among others [21] and Section 2.2.4. Traditionally, the names Bratu [17, 18] and, sometimes, Gelfand [42] are coupled to this model. However, we propose to use the name Picard as well, since we found by performing a historical literature study that he was the first one who actually introduced the model, with non-unique solutions. His report on this model appeared two decades earlier than Bratu [18]. For more information on this observation, we would like to refer to the four-page note by Picard in [107].

The classical Gelfand-Bratu (GB) problem $(N=\infty$ and $\lambda \geq 0)$ is a nonlinear elliptic (partial) differential equation, which finds, for example, its applications in combustion theory (the thermal ignition of a chemically active mixture of gasses) [11]. Other application areas of the GB problem appear in elasticity theory (membrane buckling) [120], in astronomy (gravitational equilibrium of polytropic stars and the Chandrasekhar model of the expansion of the universe) [24], in thermo-electro-hydrodynamic models and in nanotechnology (electrospinning processes) [137].

In previous numerical studies of the classical Gelfand-Bratu problem, several numerical methods have been proposed and compared to the exact solution. Most of these methods converge only to one of the two solutions of the model. Different computational techniques were compared with each other. For example, finite difference methods and multigrid methods are used in [97]. A direct shooting method and a Lie-group shooting method were presented in [21, 1]. Further, perturbation iterations, parameter perturbations and parameter splines were implemented in [73]. Phase plane solutions for perturbation problems were given in [102]. Boyd's method [14] succeeded in giving both the lower and upper solutions, in case of multiple solutions. Buckmire [21] applied Mickens' nonstandard finite difference method (NSFD) and compared the performances of the Adomian decomposion method, Boyd's pseudospectral method, the nonlinear

This chapter is based on the article published as [148]: P. A. Zegeling and S. Iqbal, Journal of Applied Mathematics and Computation, 2018, 324: 266-284. 
shooting method and standard finite difference (SFD) and NSFD methods. Buckmire reported that the NSFD method may converge to both solutions (the lower and the upper one) and is more accurate than SFD. A smart NSFD scheme for second order nonlinear boundary value problem has been discussed in Erdogan [35]. The related, more general, compact exponentially fitted method is used in [99] and SFD and NSFD approaches are considered as special cases. Recently an iterative finite difference method for solving the GB model has been discussed in [128]. Mohsen [96] presented a straightforward solution technique of the 1D planar Bratu problem with different treatments of the resulting nonlinear system of equations by using SFD and NSFD methods. Mohsen recommended a simple sinusoidal function as an initial guess for NSFD which provides more accurate results.

Motivated by the recent articles of Mohsen [96] and Buckmire [21], we present a new and extended study of the tBP model of which the GB model is a special case. We consider several cases of model (2.1) and investigate properties of the solution $u_{\lambda}^{N}(x)$ which depend on the parameter $\lambda$ and the index $N$. We present several theoretical properties of the solution in which each solution of the model (2.1) has exactly one maximum and is symmetric at $x=\frac{1}{2}$ for $\lambda>0$. These previous articles considered only positive solutions but we consider all smooth solutions, where some of them are periodic and others are semi-periodic. We also show theoretically and numerically that a unique solution exists for $\lambda \leq 0$. We work out asymptotic expressions, to show the behavior of the solution for small and large values of the parameter $\lambda$. We present a further study of the NSFD scheme for iteratively solving the resulting nonlinear systems by choosing a simple sinusoidal function having the appropriate amplitude, as an initial guess. We observe that NSFD has a similar simplicity as an SFD approximation but it is slightly more accurate, in most cases. Numerical experiments show that a large number of solutions can be obtained which are either periodic or semi-periodic. In fact, the theory shows that infinitely many may exist. This is explored in an upcoming article [146]. Graphically we also present the bifurcation nature of all possible cases of the tBP model (2.1). In literature, mainly positive solutions are considered for the $\lambda \geq 0$-case. In this chapter, we give a full classification of all solution types of model (2.1), both positive or negative and (semi)-periodic, for all $\lambda \in \mathbb{R}$.

The chapter is organized as follows. In section 2.2, we present several theoretical, analytical and asymptotic properties of the solution and we also discuss exact solutions for some special cases of the truncated Bratu-Picard model (2.1). The SFD and NSFD approximations are worked out in section 2.3. In section 2.4, numerical experiments are performed to discuss the numerical aspects of the different cases of the truncated Bratu-Picard problem. All types of possible shapes of solutions (periodic and semi-periodic) and bifurcations are displayed in section 2.5. In section 2.6, we summarize the theoretical and numerical results.

\subsection{Properties of the solution}

We will denote solutions of the model (2.1) simply by $u(x)$ or, when it is appropriate, by $u_{\lambda}^{N}(x)$ to stress their dependence on the parameters $\lambda$ and $N$.

\subsubsection{General properties}

We distinguish between several cases. For this, we define the following two subsets of $\mathbb{N}$ :

$\mathcal{N}_{2}:=\{2,4,6,8, \ldots\}$,

$\mathcal{N}_{3}:=\{3,5,7,9,11, \ldots\}$.

Note, that the special cases $N=0, N=1$ and $N=\infty$ will be treated separately. Further, it is 
useful to define the functions

$$
f(u)=f_{N}(u):=\sum_{n=0}^{N} \frac{u^{n}}{n !} \text { and } F(u):=\int_{0}^{u} f(\omega) d \omega .
$$

In the following part, we describe and prove a series of analytical and asymptotic properties of the solution $u(x)$ of model (2.1).

\section{Lemma 1}

For $N \in \mathcal{N}_{2}$, we have $f_{N}(u)>0$ for all $u \in \mathbb{R}$, whereas for $N \in \mathcal{N}_{3}, f_{N}(u)>0$ for all positive $u$ and a unique value $\tilde{u}<0$ exists with $f_{N}(\tilde{u})=0$ (see also the four graphs in Figure 2.1).

Proof: Note that, for $u \geq 0$, automatically we find $f_{N}(u)>0$, since all single terms are positive and $f_{N}(0)=1$. Next, assume that $u<0$. Then $f_{N}^{\prime}(u)=f_{N-1}(u)$ and $f_{N}(u)=$ $f_{N-1}(u)+\frac{u^{N}}{N !}$. For $N \in \mathcal{N}_{2}$, we observe that $\lim _{u \rightarrow-\infty} f_{N}(u)=+\infty$. Suppose that there exists a value $\tilde{u}<0$ such that $f_{N}(\tilde{u}) \leq 0$. Then, there must be a $u^{*}<0$ such that $f_{N}\left(u^{*}\right) \leq 0$ and $f_{N}^{\prime}\left(u^{*}\right)=0$. At this minimum at $u=u^{*}$, we find:

$$
f_{N}\left(u^{*}\right)=f_{N}^{\prime}\left(u^{*}\right)+\frac{\left(u^{*}\right)^{N}}{N !}=\frac{\left(u^{*}\right)^{N}}{N !}>0 .
$$

This contradiction shows that $f_{N}(u)>0$ for $u<0$. Therefore, $f(u)=f_{N}(u)>0, \quad \forall u \in$ $\mathbb{R}, N \in \mathcal{N}_{2}$. For the second part, suppose that $N \in \mathcal{N}_{3}$. Then $N-1$ is even and, as before, $f_{N}^{\prime}(u)=f_{N-1}(u)$. Since $\lim _{u \rightarrow-\infty} f_{N}(u)=-\infty$, we conclude that there is a unique $\tilde{u}<0$ such that $f_{N}(\tilde{u})=0$.

\section{Lemma 2}

For $N \in \mathcal{N}_{2}$, each solution $u(x)$ of model (2.1) is positive for $\lambda>0$, and it is negative for $\lambda<0$, respectively.

Proof: From Lemma 1 and the fact that $\lim _{u \rightarrow 0} \frac{u}{\sum_{n=0}^{N} \frac{u^{n}}{n !}}=0$ and $\lim _{u \rightarrow \infty} \frac{\sum_{n=0}^{N} \frac{u^{n}}{n !}}{u}=\infty$, we obtain the results in Section 3.1 in reference [3], that $u(x) \geq 0$ (for the case $\lambda>0$ ). For the case $\lambda<0$, we write $\bar{\lambda}:=-\lambda>0$ and $\bar{u}:=-u$. Model (2.1) can then be written as:

$$
\bar{u}^{\prime \prime}+\bar{\lambda} \sum_{n=0}^{N} \frac{(-1)^{n} \bar{u}^{n}}{n !}=0 .
$$

It is easily seen, using a similar proof as in Lemma 1 (cf. Figure 2.1, upper plot on the right), that $f(\bar{u})=\sum_{n=0}^{N} \frac{(-1)^{n} \bar{u}^{n}}{n !}$ is positive for $N \in \mathcal{N}_{2}$. Again, using Section 3.1 in [3], we conclude that $\bar{u}(x) \geq 0$, and, therefore, $u(x)=-\bar{u}(x) \leq 0$ for $\lambda \leq 0$.

\section{Lemma 3}

For $N \in \mathcal{N}_{2} \cup\{\infty\}$, each solution $u(x)$ of model (2.1), if it exists, has exactly one maximum for $\lambda>0$ and, in the case of $\lambda<0$, one minimum.

Proof (we use elements from [7]): The trivial solution $u(x) \equiv 0, \forall x \in[0,1]$ does not satisfy model (2.1), because $f(0)=1$. This implies that $u(x)$ must have maximum (and/or minimum) values on the interval $x \in[0,1]$. Assume, that this happens at a point $x=x_{0} \in[0,1]$. Then 

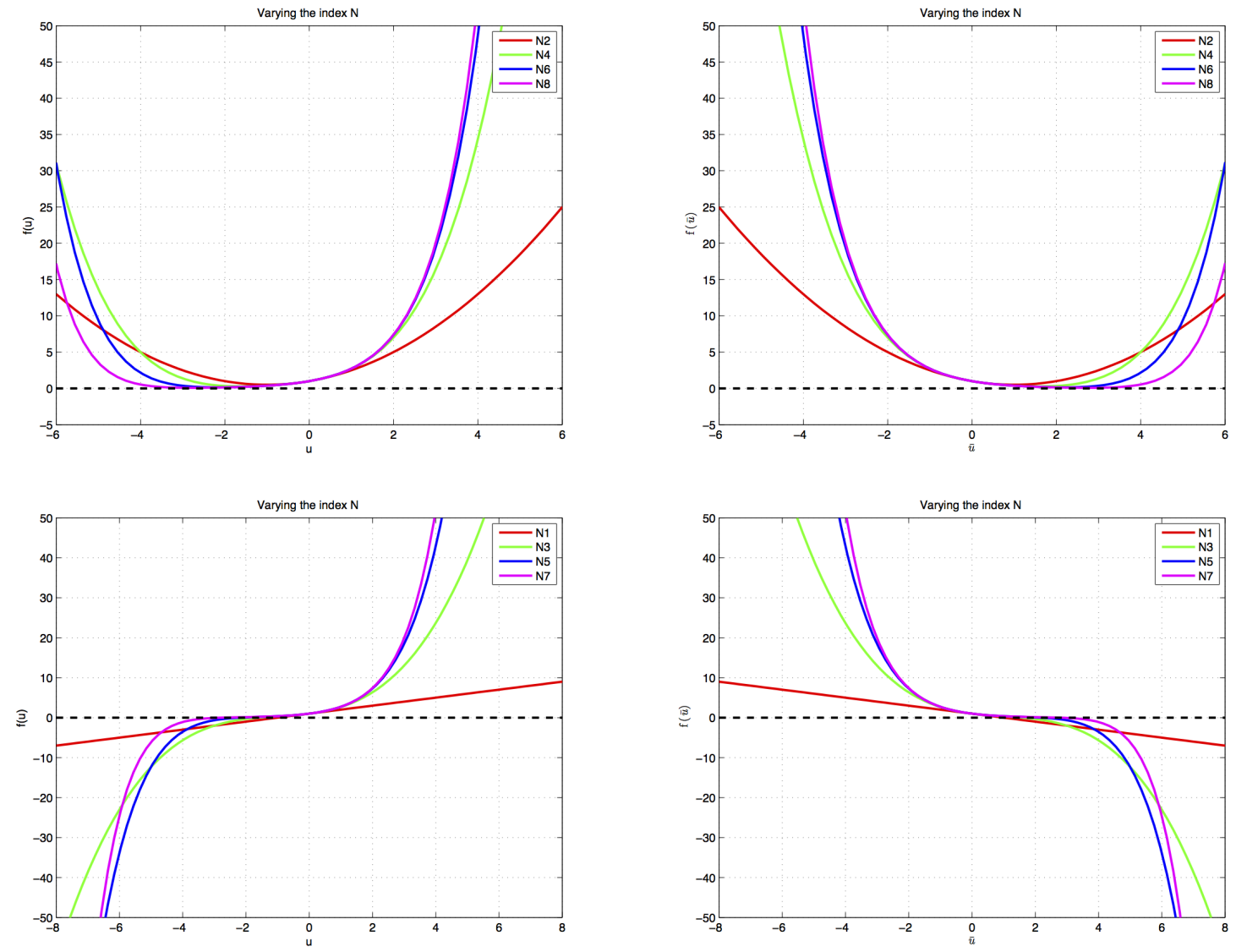

Figure 2.1: The functions $f(u)$ and $f(\bar{u})=f(-u)$, for $N \in \mathcal{N}_{2}$ (upper two plots) are positive and for $N \in \mathcal{N}_{3}$ (lower two plots) they are both positive and negative. 
$u^{\prime}\left(x_{0}\right)=0$ and also $u^{\prime \prime}\left(x_{0}\right)=-\lambda f\left(x_{0}\right)$. For $\lambda>0$, we find $u^{\prime \prime}\left(x_{0}\right)<0$, since $f\left(x_{0}\right)>0$. Thus, we have a maximum at $x=x_{0}$. As $u^{\prime \prime}(x)<0$ for all $x \in[0,1]$, there is no minimum and there can not occur further maxima: the unique maximum is found at $x=x_{0}$. In the case $\lambda<0$, a similar proof, using the fact that then $u^{\prime \prime}>0$, shows the existence of a unique minimum at $x=x_{0}$.

\section{Lemma 4}

For $N \in \mathcal{N}_{2} \cup\{\infty\}$, each solution $u(x)$ of model (2.1) attains its maximum value at $x=\frac{1}{2}$ for $\lambda>0$ (and its minimum value for negative $\lambda$ ). For $\lambda \in \mathbb{R}$, the solution is symmetric around $x=\frac{1}{2}$ in the following sense: $u(x)=u(1-x)$.

Proof (we follow a proof similar to the one in [76]): First, we treat the case $\lambda>0$. From Lemma 3 a unique maximum is obtained at $x=x_{0}$, i.e. $\|u\|_{\infty}=u\left(x_{0}\right)$. It is obvious that $u^{\prime}(x) \geq 0$ on $\left[0, x_{0}\right]$ and $u^{\prime}(x) \leq 0$ on $\left[x_{0}, 1\right]$. Integrating equation (2.1), we obtain:

$$
\frac{1}{2}\left[u^{\prime}(x)\right]^{2}+\lambda F(u(x))=\lambda F\left(\|u\|_{\infty}\right),
$$

where we have used the constraints $F\left(u\left(x_{0}\right)\right)=F\left(\|u\|_{\infty}\right)$ and $u^{\prime}\left(x_{0}\right)=0$. Integrating once more, we find:

and

$$
\sqrt{2 \lambda} x=\int_{0}^{u} \frac{d \omega}{\sqrt{F\left(\|u\|_{\infty}\right)-F(\omega)}} \text {, for } 0 \leq x \leq x_{0},
$$

$$
\sqrt{2 \lambda}(1-x)=\int_{0}^{u} \frac{d \omega}{\sqrt{F\left(\|u\|_{\infty}\right)-F(\omega)}}, \text { for } x_{0} \leq x \leq 1 .
$$

Setting $x=x_{0}$ and $u\left(x_{0}\right)=\|u\|_{\infty}$, it follows that $x_{0}$ must be the point $\frac{1}{2}$ and that $u(x)=u(1-x)$, i.e., any solution of (2.1) is symmetric around the point $x=\frac{1}{2}$. The case $\lambda<0$ is treated similarly, with the maximum replaced by the minimum.

\section{Lemma 5}

The solution $u(x)$ of model (2.1) is unique for $\lambda \leq 0$ and for any value of $N \in \mathbb{N}$.

Proof (this is a modified version of the proof in [52]): Suppose that there exist two solutions: $u_{1}(x)$ and $u_{2}(x)$ with $u_{1}^{\prime}(0) \neq u_{2}^{\prime}(0)$. It is no restriction to set $u_{1}^{\prime}(0)>u_{2}^{\prime}(0)$. Using the continuity of the solutions, we have $u_{1}^{\prime}(x)>u_{2}^{\prime}(x)$ on some interval $x \in\left[0, x_{1}\right]$. Suppose that at $x=x_{1}$ : $u_{1}^{\prime}\left(x_{1}\right)=u_{2}^{\prime}\left(x_{1}\right)$. At this point, we have

$$
u_{1}^{\prime \prime}\left(x_{1}\right)=\lim _{h \downarrow 0} \frac{u_{1}^{\prime}\left(x_{1}\right)-u_{1}^{\prime}(x-h)}{h} \text { and } u_{2}^{\prime \prime}\left(x_{1}\right)=\lim _{h \downarrow 0} \frac{u_{2}^{\prime}\left(x_{1}\right)-u_{2}^{\prime}\left(x_{1}-h\right)}{h} .
$$

Then:

$$
u_{2}^{\prime \prime}\left(x_{1}\right)-u_{1}^{\prime \prime}\left(x_{1}\right)=\lim _{h \downarrow 0} \frac{u_{1}^{\prime}\left(x_{1}-h\right)-u_{2}^{\prime}\left(x_{1}-h\right)}{h} \geq 0,
$$

since $h>0$ and $u_{1}^{\prime}(x)>u_{2}^{\prime}(x)$ on $\left[x_{1}-h, x_{1}\right]$. From this follows: $u_{2}^{\prime \prime}\left(x_{1}\right) \geq u_{1}^{\prime \prime}\left(x_{1}\right)$. Next, write:

$$
u_{1}(x)=\int_{0}^{x} u_{1}^{\prime}(\omega) d \omega \text { and } u_{2}(x)=\int_{0}^{x} u_{2}^{\prime}(\omega) d \omega .
$$

This yields: $u_{2}\left(x_{1}\right)=\int_{0}^{x_{1}} u_{2}^{\prime}(\omega) d \omega<\int_{0}^{x_{1}} u_{1}^{\prime}(\omega) d \omega=u_{1}\left(x_{1}\right)$. Note that $f^{\prime}(u)>0$, which gives $f\left(u_{1}\left(x_{1}\right)\right)>f\left(u_{2}\left(x_{1}\right)\right)$, as $u_{1}\left(x_{1}\right)>u_{2}\left(x_{1}\right)$. This makes a contradiction, because

$$
u_{2}^{\prime \prime}\left(x_{1}\right)=f\left(u_{2}\left(x_{1}\right)\right)<f\left(u_{1}\left(x_{1}\right)\right)=u_{1}^{\prime \prime}\left(x_{1}\right) .
$$

Conclusion: there is only one solution. 


\section{Lemma 6}

For $0<\lambda \ll 1$, we find for any value of $N \in \mathbb{N}$ the following asymptotic expressions for the solution with the lowest maximum: $u(x)=\frac{\lambda}{2} x(1-x)+\frac{1}{24} x\left(1-2 x^{2}+x^{3}\right) \lambda^{2}+\mathcal{O}\left(\lambda^{3}\right)$ and $\|u\|_{\infty}=\frac{\lambda}{8}+\frac{5}{384} \lambda^{2}+\mathcal{O}\left(\lambda^{3}\right)$.

Proof: Assume that the solution can be written as an asymptotic series in the (small) parameter $\lambda$ :

$$
u(x)=u_{0}(x)+\lambda u_{1}(x)+\lambda^{2} u_{2}(x)+\ldots
$$

Substituting this series in equation (2.1) and collecting the different terms for the powers of $\lambda$, we find:

$$
\left\{\begin{array}{l}
\text { for } \lambda^{0}: u_{0}(x)=0 \\
\text { for } \lambda^{1}: u_{1}(x)=\frac{1}{2} x(1-x) \\
\text { for } \lambda^{2}: u_{2}(x)=\frac{1}{24} x\left(1-2 x^{2}+x^{3}\right) \\
\ldots
\end{array}\right.
$$

The results follow from equations (2.2) and by taking the maximum norm (see also the book by Verhulst [135] for more details).

\section{Lemma 7}

For $0<\lambda \ll 1$ and $N=2$, two solutions exist of which the lower solution is $u(x)=\mathcal{O}(\lambda)$ and the upper solution is $u(x)=\mathcal{O}\left(\frac{1}{\lambda}\right)$.

Proof: Write $u(x)=\lambda^{\beta} v(x)$ with $v(x)=\mathcal{O}(1)$ and $\beta \in \mathbb{R}$ to be determined (note that, in such a way, we cover all possible scales in $u$ ). Substituting this into model (2.1) with $N=2$, we obtain

$$
\lambda^{\beta} v^{\prime \prime}+\lambda+\lambda^{\beta+1} v+\frac{1}{2} \lambda^{2 \beta+1} v^{2}=0 .
$$

Significant degenerations (see Eckhaus [34], Verhulst [135]) appear only for the values $\beta=1$ and $\beta=-1$. The case $\beta=1$ is exactly the solution already obtained in Lemma 6 , which is $\mathcal{O}(\lambda)$ and characterizes the 'lower' solution of the two. For the case $\beta=-1$, we find

$$
v^{\prime \prime}+\lambda^{2}+\lambda v+\frac{1}{2} v^{2}=0
$$

Substituting the asymptotic series $v=v_{0}+\lambda v_{1}+\lambda^{2} v_{2}+\ldots$ and collecting terms of equal order, we find, for $\lambda^{0}$ :

$$
v_{0}^{\prime \prime}+\frac{1}{2} v_{0}^{2}=0, v_{0}(0)=v_{0}(1)=0 .
$$

From this differential equation, one could extract $v_{0}$ (a unique solution $v_{0}$ exists, see [145], p. 241, case 2), and similarly for the $\lambda^{1}$ terms, $v_{1}$, etcetera. Indeed, this then would give the asymptotic solution:

$$
u(x)=\frac{1}{\lambda} v_{0}(x)+v_{1}(x)+\lambda v_{2}(x)+\ldots=\mathcal{O}\left(\frac{1}{\lambda}\right) .
$$

This is the second ('upper') solution. No other degenerations (values of $\beta$, or scales) can be found, so that one can conclude that two solutions exist for $\lambda \ll 1$.

The results might be extended to other values of $N$. However, for $N>2$, the number of significant degenerations increases significantly. It is not obvious which of those degenerations should be used, in general. 


\section{Lemma 8}

For $\lambda \gg 1$ and $N=2$, no strictly positive solution exists for model (2.1). Proof: Define $\epsilon:=\frac{1}{\lambda} \ll 1$. Then model (2.1) can be rewritten as:

$$
\epsilon u^{\prime \prime}+1+u+\frac{1}{2} u^{2}=0, u(0)=u(1)=0,0<\epsilon \ll 1 .
$$

We now write the asymptotic series as $u=u_{0}+\epsilon u_{1}+\epsilon^{2} u_{2}^{2}+\ldots$. The terms for $\epsilon^{0}$ give: $1+u_{0}+\frac{1}{2} u_{0}^{2}=$ 0 which has no solution, since $1+u_{0}+\frac{1}{2} u_{0}^{2}$ is always positive. Therefore, we can not find $u_{0}$, and neither the functions $u_{1}, u_{2}, \ldots$, since they can only follow from (the non-existent) $u_{0}$. To cover all scales, we introduce $u(x)=\epsilon^{\gamma} w(x)$ with $w(x)=\mathcal{O}(1)$ and $\gamma \in \mathbb{R}$. The only significant degenerations are $\gamma=1$ and $\gamma=-1$ (see [34]). However, when calculating the first order term, these two values of $\gamma$ yield contradictions as well. The conclusion is that for $\lambda \gg 1$ no solutions exist.

\section{Conjecture 9}

For $\lambda>0$ and for $N \in \mathcal{N}_{3}$, infinitely many solutions $u(x)$ for model (2.1) exist. All solutions are either periodic or semi-periodic. We give numerical evidence in section 2.4 to support this conjecture. A recent theoretical proof can be found in [146].

\subsubsection{Exact solutions for $N=0$}

For $N=0$, equation (2.1) reduces to:

$$
u^{\prime \prime}(x)+\lambda=0
$$

and we can directly solve it. The solution reads:

$$
u_{\lambda}^{0}(x)=\frac{\lambda}{2} x(1-x)
$$

with a maximum value of $\left\|u_{\lambda}^{0}\right\|_{\infty}=\frac{|\lambda|}{8}$. In the phase plane $\left(u, u^{\prime}\right)$ no critical points are present (see Figure 2.2) .

\subsubsection{Exact solutions for $N=1$}

For $N=1$, model $(2.1)$ is linear:

$$
u^{\prime \prime}(x)+\lambda(1+u(x))=0 .
$$

We have to distinguish between three different subcases. For $\lambda<0$, the solution has the form:

$$
u(x)=u_{\lambda}^{1}(x)=-1+\frac{1}{\mathrm{e}^{\sqrt{-\lambda}}-\mathrm{e}^{-\sqrt{-\lambda}}}\left[\left(1-\mathrm{e}^{-\sqrt{-\lambda}}\right) \mathrm{e}^{\sqrt{-\lambda} x}+\left(\mathrm{e}^{\sqrt{-\lambda}}-1\right) \mathrm{e}^{-\sqrt{-\lambda} x}\right] .
$$

Note that for $\lambda \rightarrow-\infty$ there is a horizontal asymptote: $\left\|u_{\lambda}^{1}\right\|_{\infty} \uparrow 1$. For $\lambda=0$, we find the trivial solution $u_{\lambda}(x) \equiv 0$, and, for $\lambda>0, \lambda \neq(m \pi)^{2}$, we obtain:

$$
u(x)=u_{\lambda}^{1}(x)=-1+\cos (\sqrt{\lambda} x)+\frac{1-\cos (\sqrt{\lambda})}{\sin (\sqrt{\lambda})} \sin (\sqrt{\lambda} x) .
$$

For $\lambda=(m \pi)^{2}(m \in\{2,4,6, \ldots\})$, there exist infinitely many solutions satisfying the differential equation and the two boundary conditions: $u_{\lambda}^{1}(x)=\sin (m \pi x)$, whereas for $\lambda=(m \pi)^{2}(m \in$ 

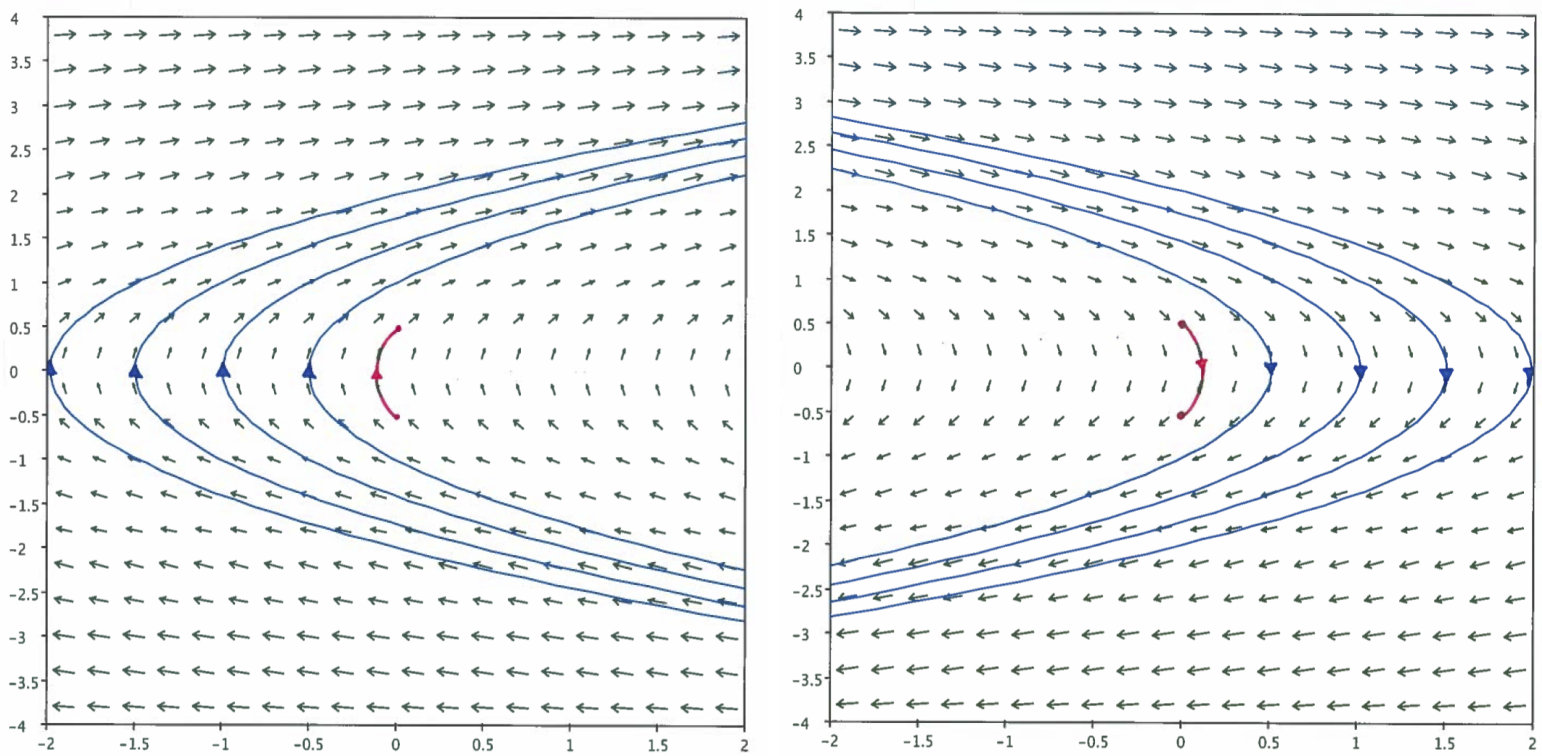

Figure 2.2: The phase plane $\left(u, u^{\prime}\right)$ for $N=0$ with $\lambda<0$ (left) and $\lambda>0$ (right): no critical points are present. The red curves represent solutions satisfying the two boundary conditions.

$\{3,5,7, \ldots\})$ no continuous solutions exist. These solutions seem to have singularities. Only for $\lambda \rightarrow(m \pi)^{2}$ with $m \in\{1,3,5, \ldots\}$ we find $\left\|u_{\lambda}^{1}\right\|_{\infty} \rightarrow \infty$. For the other values of $m$, i.e. $m \in$ $\{2,4,6, \ldots\}$, there is no singularity at $\lambda=(m \pi)^{2}$, which can be checked easily using a Taylor series. The behaviour of $\left\|u_{\lambda}^{1}\right\|_{\infty}$ as a function of $\lambda$ can be found in section 2.5. In the phase-plane $\left(u, u^{\prime}\right)$, the model possesses a critical point to the left of the solution trajectory: a saddle point in the left half plane for $\lambda<0$, whereas it has a center point for positive $\lambda$-values (see Figure $2.3)$.
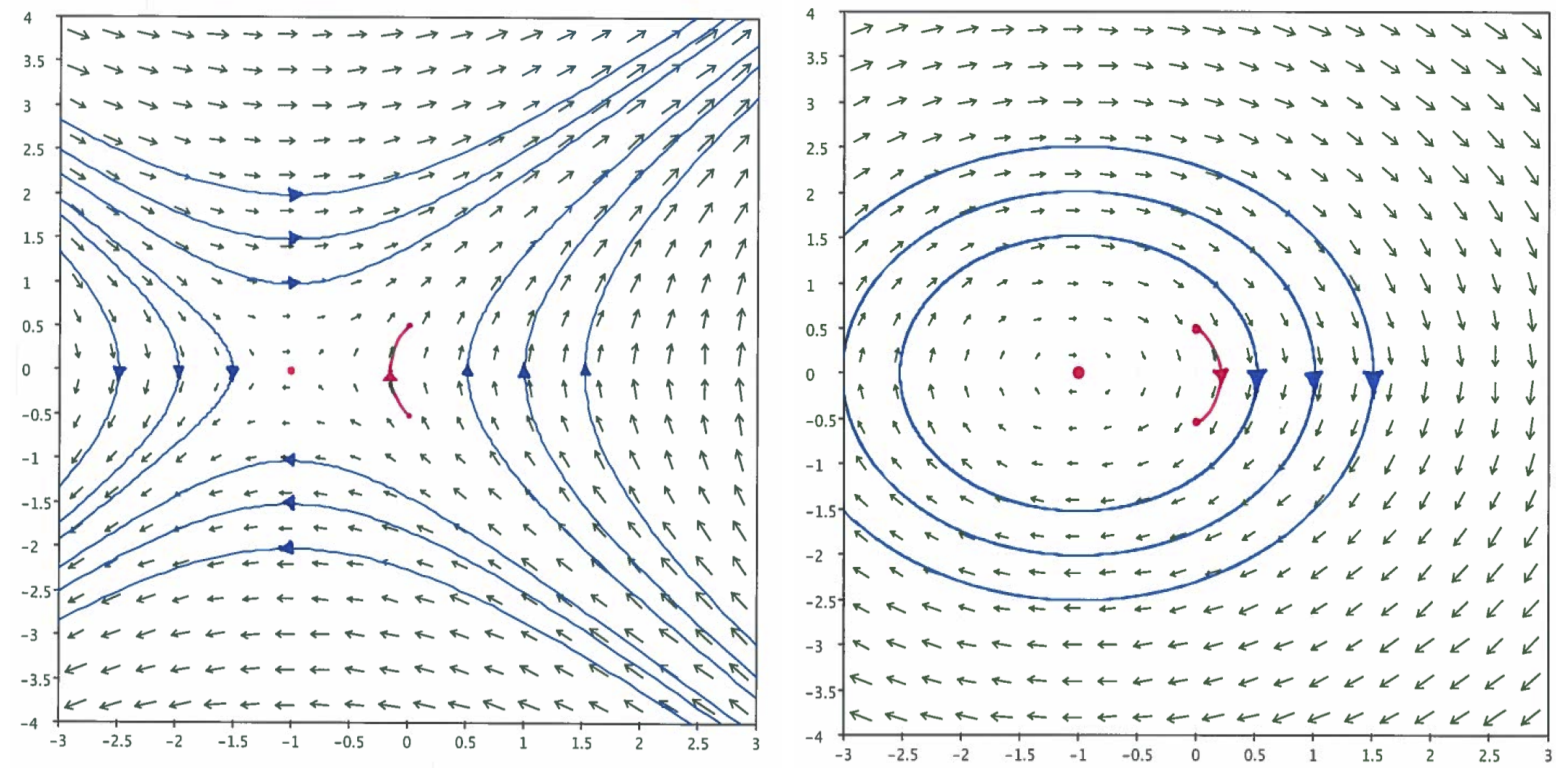

Figure 2.3: The phase plane $\left(u, u^{\prime}\right)$ for $N=1$ with $\lambda<0$ (left) and $\lambda>0$ (right): a saddle point (left) and a center point (right) appear in this case, as indicated by the red bullets. The red curves represent solutions satisfying the two boundary conditions. 


\subsubsection{Exact solutions for $N=\infty$}

For $N=\infty$, the second term in (2.1) becomes the Taylor series of the exponential function $\mathrm{e}^{u(x)}$ around zero. We then arrive at the 'standard' Gelfand-Bratu model:

$$
\left\{\begin{array}{l}
u^{\prime \prime}(x)+\lambda \mathrm{e}^{u(x)}=0, \quad x \in[0,1], \quad \lambda \in \mathbb{R}, \\
u(0)=u(1)=0 .
\end{array}\right.
$$

For positive $\lambda$ (see, for instance, Buckmire [21]), the exact solution is characterized by the following expression:

$$
\left\{\begin{array}{l}
u(x)=u_{\lambda}^{\infty}(x)=-2 \ln \left[\frac{\cosh \left(\left(x-\frac{1}{2}\right) \frac{\theta}{2}\right)}{\cosh \left(\frac{\theta}{4}\right)}\right], \\
\theta=\sqrt{2 \lambda} \cosh \left(\frac{\theta}{4}\right) .
\end{array}\right.
$$

It is well known that for $0<\lambda<\lambda_{c}$ there exist exactly two solutions to model (2.8), precisely one solution for $\lambda=\lambda_{c}$ and no solutions exist for $\lambda>\lambda_{c}$. Figure 2.4 illustrates the possible solutions. The value for $\lambda_{c}=3.513830719 \ldots$ can be found numerically using the second formula in equation (2.9) and applying a Newton-Raphson method as described in [21] . In literature, only solutions for positive $\lambda$ are given. Here, we would like to consider the case $\lambda<0$ as well. It

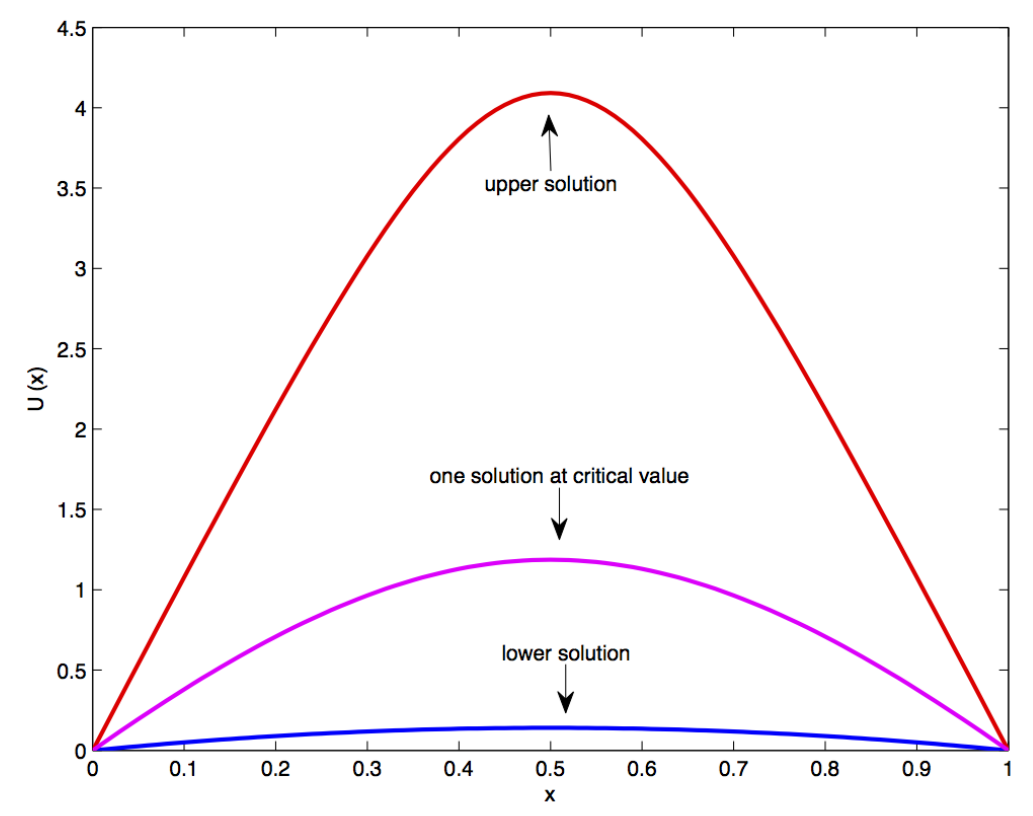

Figure 2.4: The solutions for $N=\infty$ depending on the values of $\lambda$.

can be checked that the solution then is given by:

$$
\left\{\begin{array}{l}
u(x)=u_{\lambda}^{\infty}(x)=-2 \ln \left[\frac{\cos \left(\left(x-\frac{1}{2}\right) \frac{\theta}{2}\right)}{\cos \left(\frac{\theta}{4}\right)}\right] \\
\theta=\sqrt{-2 \lambda} \cos \left(\frac{\theta}{4}\right) .
\end{array}\right.
$$

For deriving this expression, one can use the relation $\cosh (i x)=\cos (x)$, among others. Furthermore, Lemma 5 states that a unique (smooth) solution $u_{\lambda}^{\infty}(x)$ exists for this case. Figure 2.5 (left panel) gives an overview of the differences between positive and negative values of $\lambda$. The parameter $\beta$ on the horizontal axis is connected to one of the two integration constants, after 
formally integrating equation (2.8). Note that on the vertical axis (in the right panel) we display the absolute maximum value of the solution as a function of the parameter $\lambda$. In this figure the specific cases, as already discussed above, can be detected easily. Note that still additional singular solutions may occur for $\lambda<0$. For example we have, for $\lambda=-\pi^{2}$ (see [139]):

$$
u_{\lambda}^{\infty}(x)=-\ln \left(1+\cos \left[\left(\frac{1}{2}+x\right) \pi\right]\right)
$$

which blows up at $x=\frac{1}{2}$. In Figure 2.5 (right panel) another way of representing the different cases as a function of $\lambda$ is displayed: The green curve shows the unique solution for $\lambda<0$. The blue and red curves illustrate the two positive solutions lower and upper respectively for $0 \leq \lambda \leq \lambda_{c}$ and no solution for $\lambda>\lambda_{c}$.
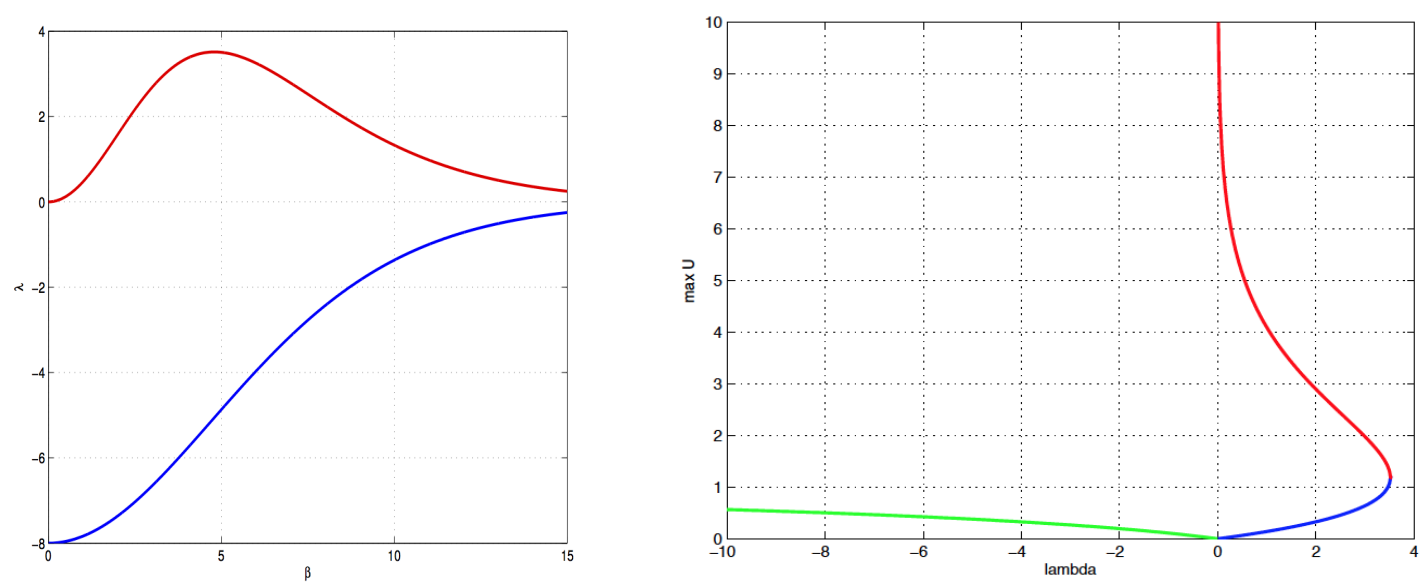

Figure 2.5: In the left frame, we display the difference between unique solutions for negative $\lambda$ (blue curve) and the three possible cases for positive $\lambda$ (red curve) depending on the parameter $\beta$ as explained in the text. In the right frame a bifurcation diagram for $N=\infty$ is given. Here, the blue and red lines indicate the two solutions for each positive $\lambda<\lambda_{c} \approx 3.514$ and the green line depicts the unique solution for all negative $\lambda$, respectively.

\subsubsection{The case: $N \in \mathcal{N}_{2}$}

As a characteristic example we take $N=2$ :

$$
u^{\prime \prime}(x)+\lambda\left(1+u(x)+\frac{1}{2} u(x)^{2}\right)=0 .
$$

This case and all other values in $\mathcal{N}_{2}$ are topologically equivalent with the $N=\infty$ case, in the sense that there exist two, one or zero solutions $u_{\lambda}^{N}$, for positive $\lambda$, with a critical value $\lambda_{c}^{N} \approx 3.96$ for $N=2$ (its precise value in general depends, of course, on $N$ ). We obtained the bifurcation curve when we consider the positive solutions, which resembles the similar behavior of classical GB model. For negative $\lambda$ values there is a unique solution (see Lemma 5). As mentioned, similar conclusions can be made for the cases $N=4,6,8, \ldots$ Numerical solutions are given in section 2.4 .

\subsubsection{The case: $N \in \mathcal{N}_{3}$}

As characteristic examples we take $N=3$ and $N=5$ :

$$
u^{\prime \prime}(x)+\lambda\left(1+u(x)+\frac{1}{2} u(x)^{2}+\frac{1}{6} u(x)^{3}\right)=0,
$$




$$
u^{\prime \prime}(x)+\lambda\left(1+u(x)+\frac{1}{2} u(x)^{2}+\frac{1}{6} u(x)^{3}+\frac{1}{24} u(x)^{4}+\frac{1}{120} u(x)^{5}\right)=0 .
$$

These cases also possess the same properties as described for $N \in \mathcal{N}_{2} \cup \infty$, when we consider the positive solutions (lower, upper and one) only. However, we will show numerically that other solutions exist as well when we discuss both positive and negative solutions. In this situation, the model has infinitely many solutions for $\lambda>0$. These solutions are either periodic or semiperiodic. Of course, numerical calculations are only able to show a (large) finite number of these. A unique solution exists for negative $\lambda$ values (see Lemma 5). Similar conclusions can be made for the cases $N=7,9, \ldots$. Note that the theoretical proof of these observations is given in reference $[146]$.

\subsection{Numerical approximation}

In this section, two different finite difference schemes SFD and NSFD are discussed to compute the numerical solutions of the truncated Bratu-Picard problem (2.1). Both schemes are based on the discrete versions of the boundary value problem by approximating the derivatives and the boundary conditions. To compute the numerical solutions of (2.1) both schemes go with a uniformly distributed spatial grid $\Delta x$. These schemes transform problem (2.1) into a system of nonlinear equations which can then be solved by using a suitable iterative method.

\subsubsection{Standard finite differences (SFD)}

To compute numerical solutions of the truncated Bratu-Picard model (2.1), we first employ a standard finite difference (SFD) scheme by discretizing the differential equation and boundary conditions. The first step in the computation of the numerical solution of (2.1) is to subdivide the continuous spatial interval $x \in[0,1]$ with a discrete grid. For this, choose the grid points $\left\{x_{j}\right\}_{j=0}^{J}$ uniformly, i.e., $x_{j}=j \Delta x, \quad j=0, \ldots, J$ with $\Delta x=\frac{1}{J}$ and approximate the second derivative in (2.1) by the second-order approximation:

$$
u^{\prime \prime}\left(x_{j}\right) \approx \frac{u_{j+1}-2 u_{j}+u_{j-1}}{(\Delta x)^{2}}, \quad j=1, \ldots, J-1,
$$

where $u_{j} \approx u_{\lambda}^{N}\left(x_{j}\right)$. This yields the discrete nonlinear system:

$$
\frac{u_{j+1}-2 u_{j}+u_{j-1}}{(\Delta x)^{2}}+\lambda \sum_{n=0}^{N} \frac{u_{j}^{n}}{n !}=0, \quad j=1, \ldots, J-1,
$$

with $u_{0}=u_{J}=0$.

\subsubsection{Nonstandard finite differences (NSFD)}

A nonstandard finite-difference scheme (NSFD) approximates a derivative by using a denominator function and uses 'non-local' or 'off-grid' representations of expressions in the differential equation [84]. Mickens in [87] showed explicitly that one can find NSFD schemes which produce exact discrete solutions of a differential equation. Mickens' discretization of the second derivative has the general form:

$$
u^{\prime \prime}\left(x_{j}\right) \approx \frac{u_{j+1}-2 u_{j}+u_{j-1}}{\phi(\Delta x)}, \quad j=1, \ldots, J-1,
$$

where the denominator function $\phi(\Delta x)$ has the property

$$
\phi(\Delta x)=(\Delta x)^{2}+\mathcal{O}\left((\Delta x)^{4}\right)
$$


Thus, in the limit as $\Delta x \rightarrow 0$ the SFD scheme and NSFD scheme will be identical. In [84], the numerical computations are conducted using a nonstandard form of the denominator function $\phi(\Delta x)$ and a 'non-local' discretization of nonlinear terms, that will lead to improving the accuracy for finite values of $\Delta x$. A discrete version of a nonstandard finite difference method for (2.1) with $\lambda>0$ is given by

$$
\frac{u_{j+1}-2 u_{j}+u_{j-1}}{2 \ln [\cosh (\Delta x)]}+\lambda \sum_{n=0}^{N} \frac{u_{j}^{n}}{n !}=0, \quad j=1, \ldots, J-1,
$$

with $u_{0}=u_{J}=0$ or even replacing the term $\sum_{n=0}^{N} \frac{u_{j}^{n}}{n !}$ by a non-local version [84]. Note that, e.g., we could use

$$
2 \ln [\cosh (\Delta x)]=(\Delta x)^{2}+\mathcal{O}\left((\Delta x)^{4}\right),
$$

and

$$
2 \ln [\cos (\Delta x)]=(\Delta x)^{2}+\mathcal{O}\left((\Delta x)^{4}\right) .
$$

These two denominator functions come from the exact solutions $(2.9)(\lambda>0)$ and $(2.10)(\lambda<0)$, respectively. These exact solutions are symmetric and attain their maximum at $x=\frac{1}{2}$. The nonlinear terms involved in the model (2.1) could be approximated in a nonlocal way. For example in (2.11), the nonlinear term $u^{2}$ could be discretized not only by $u_{k}^{2}$ or $u_{k+1}^{2}$ but by the following:

$$
u^{2}=\left\{\begin{array}{l}
u_{k} u_{k+1} \\
u_{k-1} u_{k} \\
u_{k-1} u_{k+1} \\
u_{k}\left(\frac{u_{k+1}+u_{k}+u_{k-1}}{3}\right) \\
\ldots
\end{array}\right.
$$

In [88], Mickens provides even more types of nonlocal discretizations that may lead to improved accuracy over SFD discretizations. In our computations we did not use the nonlocal discretizations of the nonlinear term in the model, because they only showed minor positive effects.

\section{$2.4 \quad$ Numerical experiments}

To solve the nonlinear systems (2.15) and (2.18), we adopt the simple approach similar to that used by [96]. It is appropriate to start the iterations with a sinusoidal function that satisfies the boundary conditions: $u_{0}(x)=A \sin (k \pi x)$ is the initial guess with an amplitude $A$ and frequency $k$, to be specified for each case. The values of $A$ and $k$ depend on the index parameters $N$ and $\lambda$. In particular, for $\lambda>0$, to obtain the lower solutions, $A$ should be taken as $A<u_{\max }$ and for the upper solutions, we need $A>u_{\max }$, where $u_{\max }$ is an estimate of the expected maximum value in the solution.

In Figure 2.11 which shows solutions for $N=3$, we take $A=9$ and $k=2$ for the first periodic solution. Similarly, for the $N=5$ case, we take $A=13$ and $k=3$ for the first semi-periodic solution. This choice depends on the final number of oscillations that we expect in the solution. The resulting nonlinear system may then be solved iteratively by using an appropriate nonlinear solver. We use the Matlab nonlinear routine fsolve.m. We tested three cases and computed all numerical solutions with this nonlinear solver. Furthermore, we compare the results obtained from SFD and NSFD schemes and present the bifurcation behaviour of the problem graphically. 


\subsubsection{Case 1: $N=\infty$}

For this case, the exact solution of the GB model for positive and negative $\lambda$ has been discussed in detail in section 2.2.4. In this section, we present a numerical comparison between standard and nonstandard finite differences. In the limit $\Delta x \rightarrow 0$ both methods coincide. We present the maximum absolute error of SFD and NSFD results by comparing them with the exact solution. We observe that the results show an error which decreases proportionally to $(\Delta x)^{2}$. The non-standard approximation NSFD only gives more accurate results for moderate values of $J$.

Tables 2.1 (lower solution) and 2.2 (upper solution) show an error comparison between standard and non-standard finite-differences. We observe, as predicted, a second-order convergence, in which the errors in NSFD are slightly smaller than SFD. Furthermore, in Table 5.3 it is seen that approximation (2.20) yields slightly better results than (2.19) for negative $\lambda$-values, as predicted. Note that, NSFD and standard FD are equally accurate in an asymptotic sense $J \rightarrow \infty$. Further, we present the numerical solution and convergence of the lower and upper solutions, as [21] reported that NSFD converges both to the lower and the upper solution. Figure 2.6 confirms the convergence of both solutions (lower and upper).

Table 2.1: Maximum error for the lower solution and convergence ratio of the approximation as a function of the number of grid points $J$ for the case with $N=\infty$ and $\lambda=1$. It shows second order behavior.

\begin{tabular}{|c||c|c|c|c|}
\hline \hline$J$ & standard FDs (2.15) & ratio & nonstandard FDs (2.18) & ratio \\
\hline \hline 5 & $9.075 \mathrm{e}-4$ & 6.35 & $7.433 \mathrm{e}-4$ & 6.07 \\
11 & $1.427 \mathrm{e}-4$ & 4.00 & $1.223 \mathrm{e}-4$ & 3.98 \\
21 & $3.560 \mathrm{e}-5$ & 4.00 & $3.071 \mathrm{e}-5$ & 3.99 \\
41 & $8.898 \mathrm{e}-6$ & 3.99 & $7.683 \mathrm{e}-6$ & 4.00 \\
81 & $2.226 \mathrm{e}-6$ & 3.98 & $1.919 \mathrm{e}-6$ & 4.02 \\
161 & $5.591 \mathrm{e}-7$ & 3.93 & $4.773 \mathrm{e}-7$ & 4.08 \\
321 & $1.422 \mathrm{e}-7$ & 3.74 & $1.168 \mathrm{e}-7$ & 4.36 \\
641 & $3.800 \mathrm{e}-8$ & - & $2.678 \mathrm{e}-8$ & - \\
\hline
\end{tabular}

Table 2.2: Maximum error for the upper solution and convergence ratio of the approximation as a function of the number of grid points $J$ for the case with $N=\infty$ and $\lambda=1$.

\begin{tabular}{|c||c|c|c|c|}
\hline \hline$J$ & standard FDs (2.15) & ratio & nonstandard FDs (2.18) & ratio \\
\hline \hline 5 & 0.31217 & 11.15 & 0.30550 & 11.64 \\
11 & 0.02798 & 4.09 & 0.02623 & 4.05 \\
21 & 0.00684 & 4.01 & 0.00647 & 4.03 \\
41 & 0.00170 & 4.00 & 0.00160 & 3.99 \\
81 & $4.251 \mathrm{e}-4$ & 4.00 & $4.009 \mathrm{e}-4$ & 4.00 \\
161 & $1.062 \mathrm{e}-4$ & 3.99 & $1.001 \mathrm{e}-4$ & 4.01 \\
321 & $2.656 \mathrm{e}-5$ & 4.00 & $2.504 \mathrm{e}-5$ & 3.99 \\
641 & $6.639 \mathrm{e}-6$ & - & $6.260 \mathrm{e}-6$ & - \\
\hline
\end{tabular}


Table 2.3: Maximum error and convergence ratio of the approximation as a function of the number of grid points $J$ for the case with $N=\infty$ and $\lambda=-1$.

\begin{tabular}{|c||c|c|c|c|c|c|}
\hline \hline$J$ & standard FDs (2.15) & ratio & nonstandard FDs (2.19) & ratio & nonstandard FDs (2.20) & ratio \\
\hline \hline 5 & $5.870 \mathrm{e}-4$ & 6.39 & $5.428 \mathrm{e}-4$ & 6.50 & $5.028 \mathrm{e}-4$ & 6.16 \\
11 & $9.175 \mathrm{e}-5$ & 4.01 & $8.339 \mathrm{e}-5$ & 3.97 & $8.155 \mathrm{e}-5$ & 3.99 \\
21 & $2.286 \mathrm{e}-5$ & 4.00 & $2.096 \mathrm{e}-5$ & 3.94 & $2.042 \mathrm{e}-5$ & 3.99 \\
41 & $5.710 \mathrm{e}-6$ & 4.00 & $5.312 \mathrm{e}-6$ & 3.95 & $5.109 \mathrm{e}-6$ & 3.99 \\
81 & $1.427 \mathrm{e}-6$ & 4.00 & $1.342 \mathrm{e}-6$ & 3.99 & $1.277 \mathrm{e}-6$ & 3.99 \\
161 & $3.565 \mathrm{e}-7$ & 4.01 & $3.359 \mathrm{e}-7$ & 3.95 & $3.197 \mathrm{e}-7$ & 3.98 \\
321 & $8.887 \mathrm{e}-8$ & 4.04 & $8.492 \mathrm{e}-8$ & 4.00 & $8.018 \mathrm{e}-8$ & 3.99 \\
641 & $2.197 \mathrm{e}-8$ & - & $2.118 \mathrm{e}-8$ & - & $2.009 \mathrm{e}-8$ & - \\
\hline
\end{tabular}
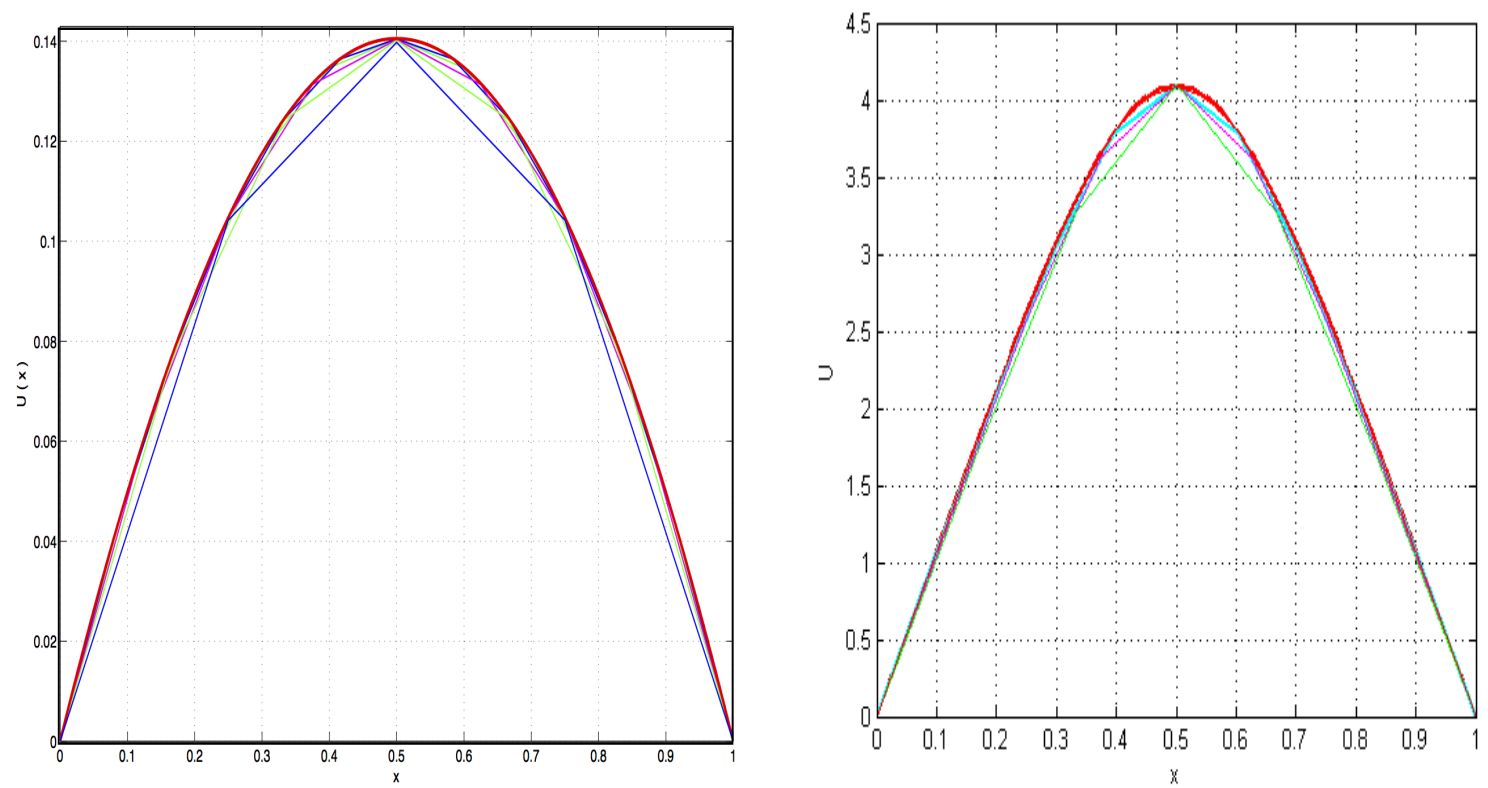

Figure 2.6: Numerical convergence behavior of NSFD for $N=\infty$. Comparison of the lower solution (left) and the upper solution (right) by increasing the number of grid points $J$ (for $\lambda=1)$. 


\subsubsection{Case 2: $N \in \mathcal{N}_{2}$}

To investigate this case, we take example (2.10) as discussed in section 2.2.5. We adopt two NSFD schemes to obtain numerical results. In NSFD1, the denominator function comes from Mickens' exact finite difference scheme [87], whereas in NSFD2 the denominator function is as derived from the exact solution already mentioned in [21] and [96]. We use the following abbreviations:

$$
N S F D 1:\left.u^{\prime \prime}(x)\right|_{x_{j}} \approx \frac{u_{j+1}-2 u_{j}+u_{j-1}}{\frac{4}{\lambda} \sin ^{2}\left(\frac{\sqrt{\lambda} \Delta x}{2}\right)}
$$

(a non-standard FD method, as described in [87,

$$
\text { NSFD2 : }\left.u^{\prime \prime}(x)\right|_{x_{j}} \approx \frac{u_{j+1}-2 u_{j}+u_{j-1}}{2 \ln (\cosh (\Delta x))}
$$

(non-standard FDs, as described in [21] and [967).

Table 2.4 gives the comparison of three FD approximations for the lower solution and $\lambda=1$. It is observed that the NSFD schemes are slightly more accurate than the SFD scheme and that the proposed denominator function in NSFD2 performs better than the originally derived one from [87]. Further, to support the result of Lemma 7, we perform a series of numerical tests for

Table 2.4: Maximum error for the lower solution, as a function of the number of grid points $J$ for the case with $N=2$ and $\lambda=1$ : a comparison between three FD schemes.

\begin{tabular}{|c||c|c|c|}
\hline \hline$J$ & SFD & NSFD1 & NSFD2 \\
\hline \hline 5 & $9.032 \mathrm{e}-3$ & $4.2993 \mathrm{e}-3$ & $7.454 \mathrm{e}-4$ \\
11 & $2.421 \mathrm{e}-3$ & $9.482 \mathrm{e}-4$ & $1.227 \mathrm{e}-4$ \\
21 & $7.543 \mathrm{e}-4$ & $2.681 \mathrm{e}-4$ & $3.080 \mathrm{e}-5$ \\
41 & $8.842 \mathrm{e}-5$ & $4.198 \mathrm{e}-5$ & $7.719 \mathrm{e}-6$ \\
81 & $2.199 \mathrm{e}-5$ & $1.048 \mathrm{e}-5$ & $1.941 \mathrm{e}-6$ \\
161 & $5.423 \mathrm{e}-6$ & $2.609 \mathrm{e}-6$ & $4.853 \mathrm{e}-7$ \\
321 & $6.357 \mathrm{e}-7$ & $1.418 \mathrm{e}-7$ & $3.186 \mathrm{e}-8$ \\
\hline
\end{tabular}

decreasing values of $\lambda$ (see Table 2.5 and Figure 2.7). We find the expected behavior as predicted by Lemma 7: for the lower solution $\left\|u_{\lambda}\right\|_{\infty}=\mathcal{O}(\lambda)$ and for the upper solution $\left\|u_{\lambda}\right\|_{\infty}=\mathcal{O}\left(\frac{1}{\lambda}\right)$. Figure 2.8 shows the upper and lower solution for $\lambda=1$ (left panel) and the bifurcation curve for positive $\lambda$ in this situation (right panel). It is similar to the case $N=\infty$, but now with $\lambda_{c} \approx 3.96$. Furthermore, Figure 2.9 shows the numerical convergence of both solutions (lower and upper) for this case. The phase plane $\left(u, u^{\prime}\right)$, for the $N=2$ case, is given in Figure 2.10. No critical points are present. The solutions satisfying the boundary conditions are represented by the red curves.

\subsubsection{Case 3: $N \in \mathcal{N}_{3}$}

In this case we discuss the two examples (2.11) and (2.12) as mentioned in section 2.2.6. These examples illustrate the fact that we expect infinitely many solutions for this case. These solutions may be periodic or semi-periodic. We again adopt the three FD schemes for comparing the numerical solutions. We consider the first semi-periodic solution for $N=3$ and $\lambda=1$ in the numerical comparison which is shown in table 2.6. This comparison illustrates that NSFD1 
Table 2.5: Maximum values for the lower and upper solution in the case $N=2$. It confirms the asymptotic analysis in Lemma 7 (see also Figure 2.7).

\begin{tabular}{|c|c|c|}
\hline \hline$\lambda$ & $\|u\|_{\infty}^{\text {lower }}$ & $\|u\|_{\infty}^{\text {upper }}$ \\
\hline \hline 0.1 & 0.01263 & $2.335 \mathrm{e}+2$ \\
0.01 & 0.00125 & $2.356 \mathrm{e}+3$ \\
0.001 & $1.250 \mathrm{e}-4$ & $2.358 \mathrm{e}+4$ \\
0.0001 & $1.250 \mathrm{e}-5$ & $2.358 \mathrm{e}+5$ \\
0.00001 & $1.249 \mathrm{e}-6$ & $2.358 \mathrm{e}+6$ \\
\hline
\end{tabular}

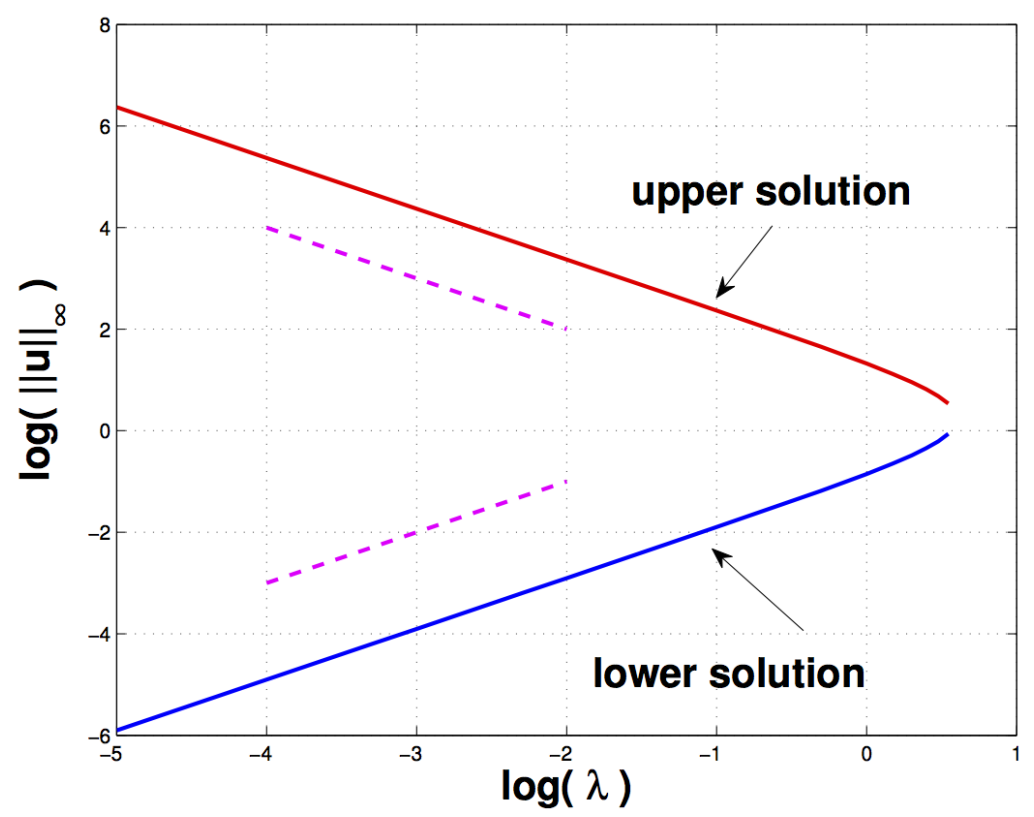

Figure 2.7: $\mathrm{A} \log _{10}-\log _{10}$ plot of the maximum value as a function of $\lambda$. The dashed lines are straight lines with slope 1 and -1 (see also Table 2.5) and indicate the asymptotic $\mathcal{O}(\lambda)$ and $\mathcal{O}\left(\lambda^{-1}\right)$ behaviour of the two solutions for $\lambda \downarrow 0$. 

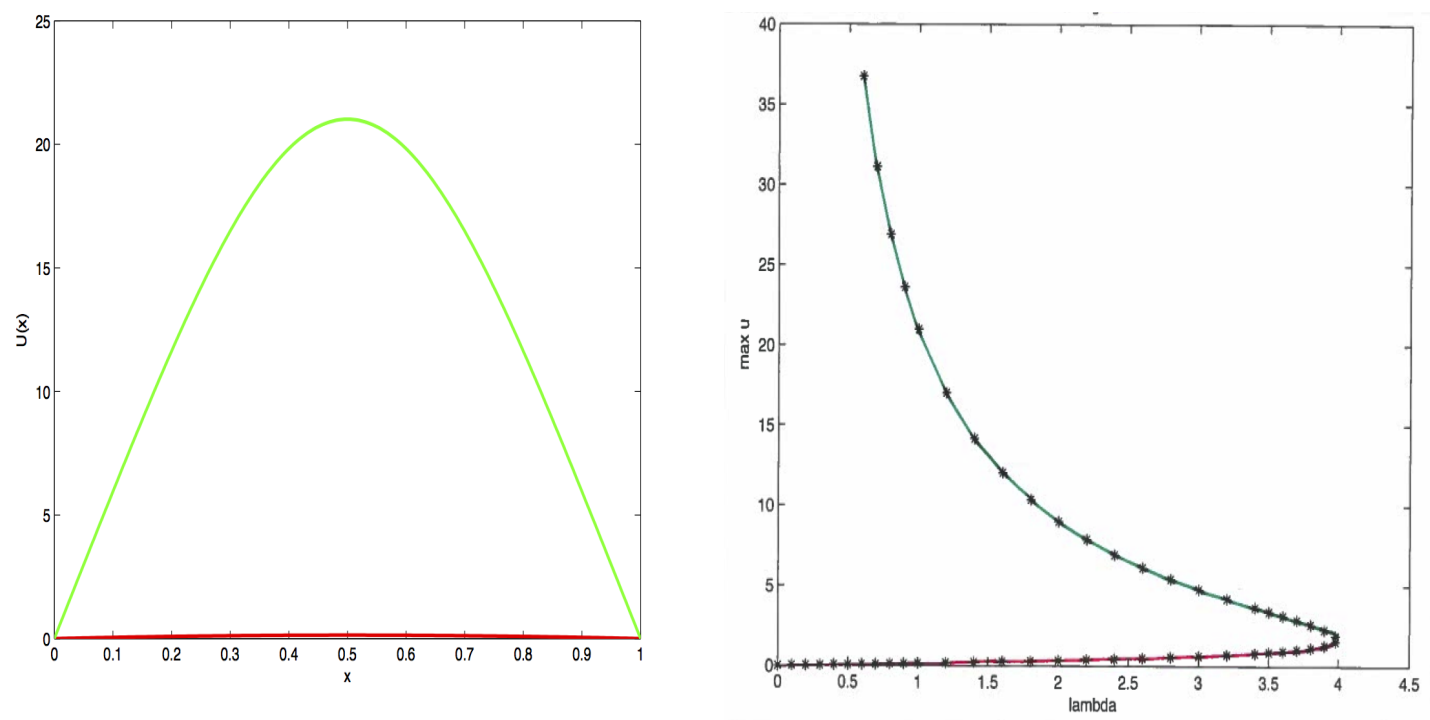

Figure 2.8: Lower (red) and upper (green) solution for $N=2$ and $\lambda=1$ (left plot). The bifurcation diagram (right plot) shows that this case resembles the $N=\infty$ case.
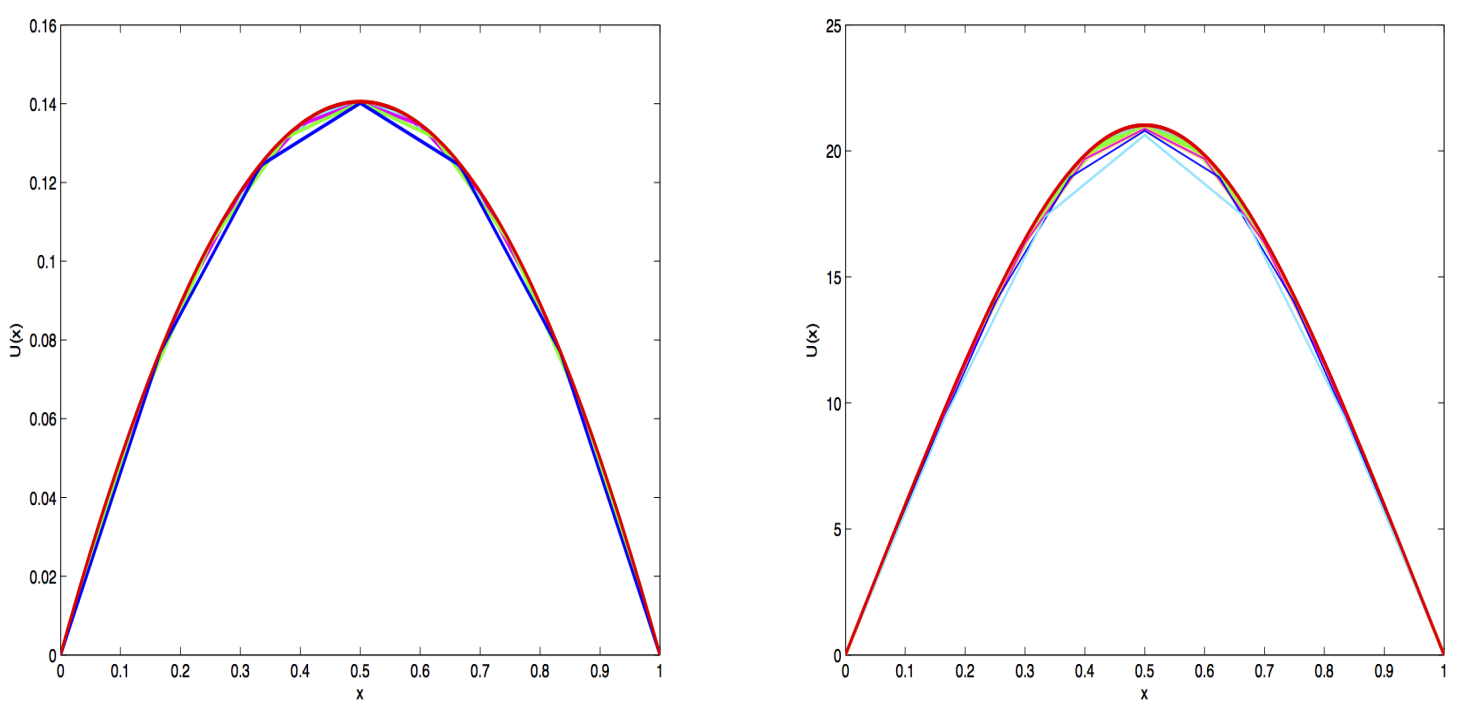

Figure 2.9: Numerical convergence using the NSFD method for the two existing solutions in the case $N=2$ : lower solution (left) and upper solution (right). 

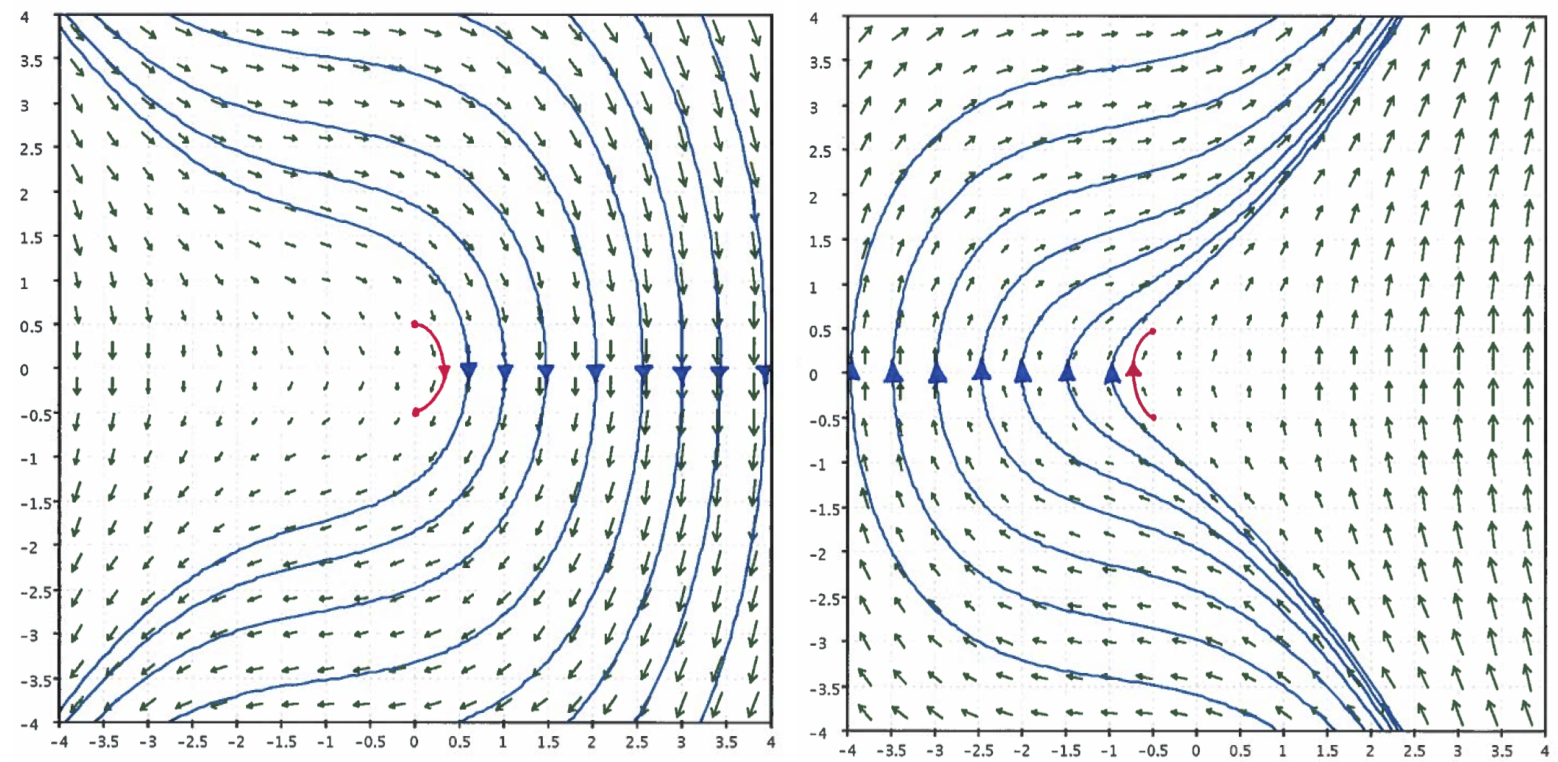

Figure 2.10: The phase plane $\left(u, u^{\prime}\right)$ for $N=2: \lambda<0$ (left) and $\lambda>0$ (right): no critical points are present. Red curves represent solutions satisfying the two boundary conditions.

is more accurate than the other two FD approximations whereas NSFD2 is slightly better than SFD. Figure 2.11 displays five (semi)-periodic solutions for $N=3$ and seven (semi)-periodic solutions for $N=5$, respectively. To characterize the bifurcation nature, we consider the relative maximum solution (consider the positive solutions) only. Figure 2.12 shows the bifurcation curve for positive $\lambda$ in which lower, upper and (semi)-periodic solution curves are displayed for this situation. Figure 2.13 confirms that the NSFD method converges to the solutions (periodic and semi-periodic) for $N \in \mathcal{N}_{3}$. Phase plane plots for this case are given in Figure 2.14, which shows the different types of solutions: periodic and semi-periodic.
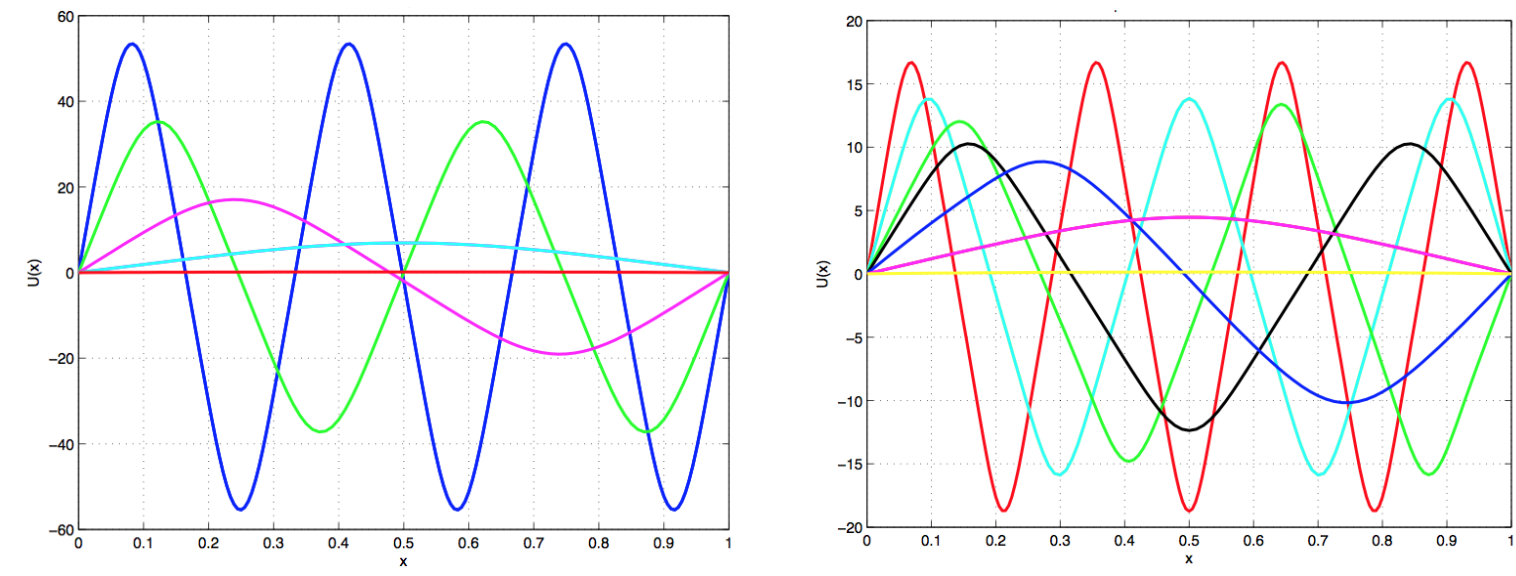

Figure 2.11: Five (semi-)periodic solutions for $N=3$ (left) and seven (semi)-periodic solutions for $N=5$ (right) with $\lambda=1$. 

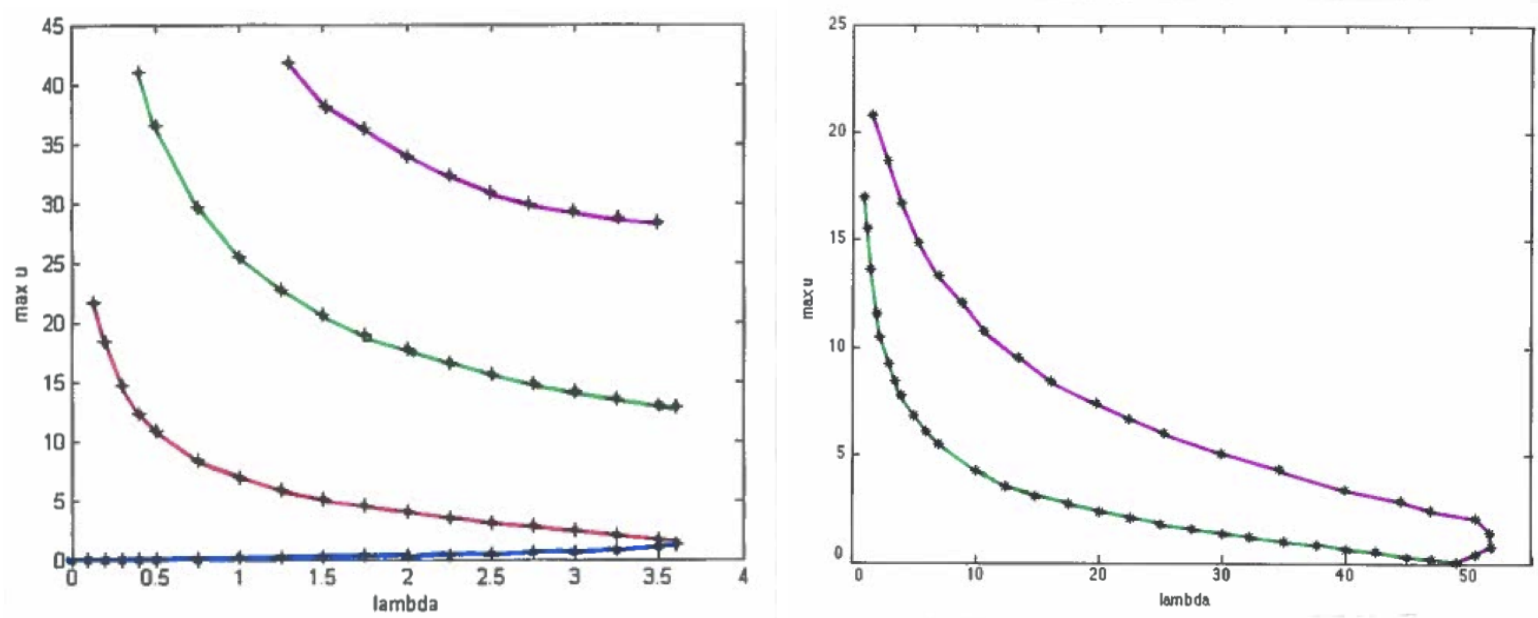

Figure 2.12: The numerical bifurcation diagram (left) for $N=3$ for positive $\lambda$. Note that only three curves are displayed. In the right frame numerical approximations of the third (green) and fourth (magenta) solution are shown fon an extended $\lambda$-interval. These results will be summarized in Figure 2.19 and theoretically explained in [146].

Table 2.6: Maximum error for the 'first' semi-periodic solution as a function of the number of grid points $J$ for the case with $N=3$ and $\lambda=1$ : a comparison between three FD schemes.

\begin{tabular}{|c||c|c|c|}
\hline \hline$J$ & SFD & NSFD1 & NSFD2 \\
\hline \hline 11 & 0.90648 & 0.07832 & 0.39244 \\
21 & 0.09455 & 0.00964 & 0.06074 \\
41 & 0.06531 & 0.00120 & 0.01436 \\
81 & 0.00836 & $1.762 \mathrm{e}-4$ & 0.00399 \\
161 & $9.811 \mathrm{e}-4$ & $8.923 \mathrm{e}-5$ & $3.321 \mathrm{e}-4$ \\
321 & $2.099 \mathrm{e}-4$ & $2.950 \mathrm{e}-5$ & $7.801 \mathrm{e}-5$ \\
\hline
\end{tabular}

Table 2.7: The dependence of the critical value $\lambda_{c}$ on the index $N$ in the model (2.1) and its accuracy as a function of the number of grid points $J$.

\begin{tabular}{|c||c|c|c|c|c|c|c|c|c|}
\hline \hline$N$ & 0 & 1 & 2 & 3 & 5 & 10 & 50 & $\ldots$ & $\infty$ \\
\hline \hline$J=21$ & - & - & 3.9970 & 3.6211 & 3.5712 & 3.5621 & 3.5283 & $\ldots$ & 3.51387 \\
\hline$J=41$ & - & - & 3.9613 & 3.6000 & 3.5649 & 3.5561 & 3.5234 & $\ldots$ & 3.51384 \\
\hline$J=81$ & - & - & 3.9600 & 3.5991 & 3.5612 & 3.5511 & 3.5224 & $\ldots$ & 3.51384 \\
\hline
\end{tabular}



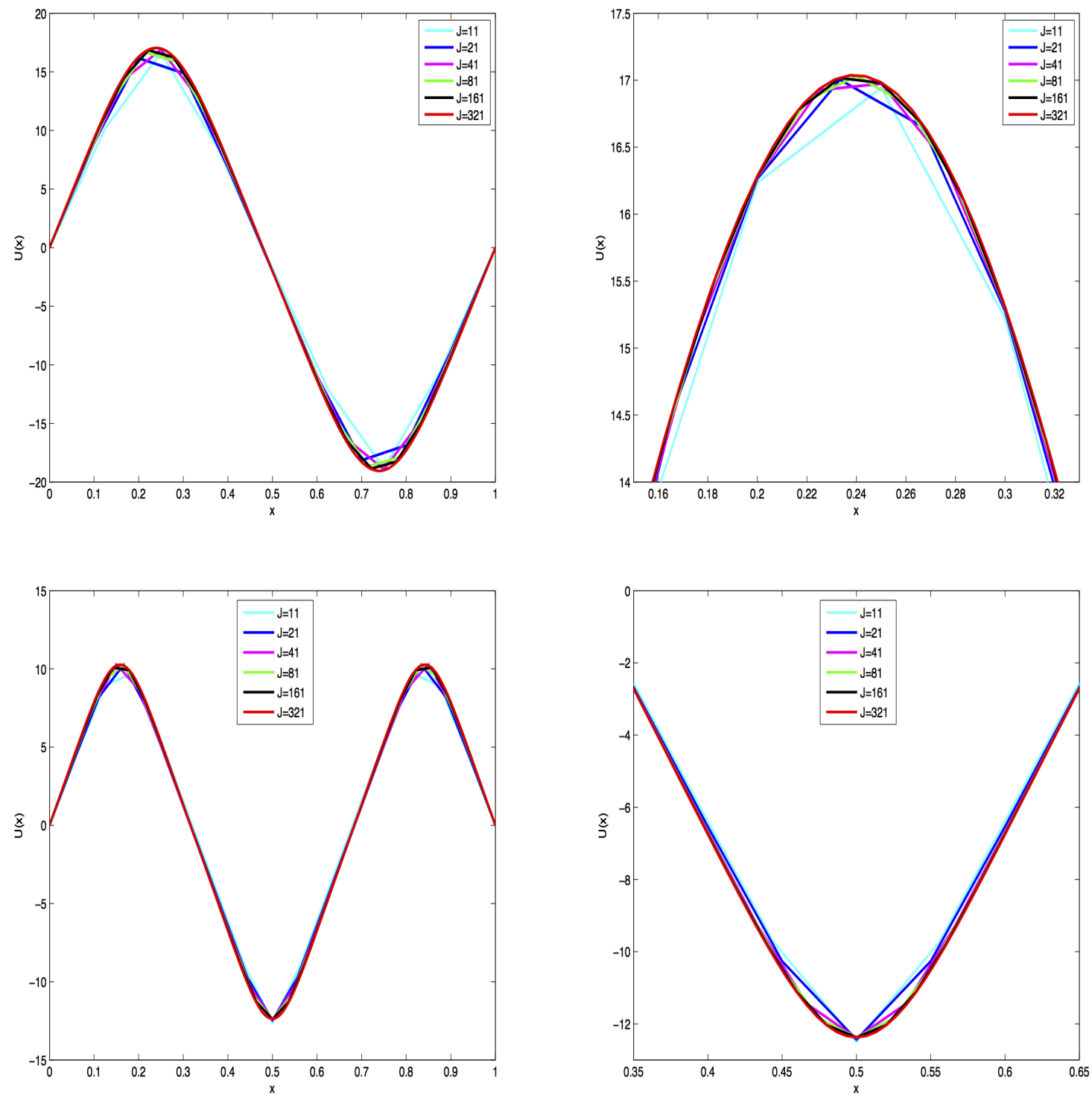

Figure 2.13: Convergence of the numerical solution using NSFD method for the first periodic solution for $N=3$ (upper left frame) and a close up near the maximum (upper right frame). Similarly, for the first semi-periodic solution for $N=5$ (lower left frame) and a close up near the minimum (lower right frame). 

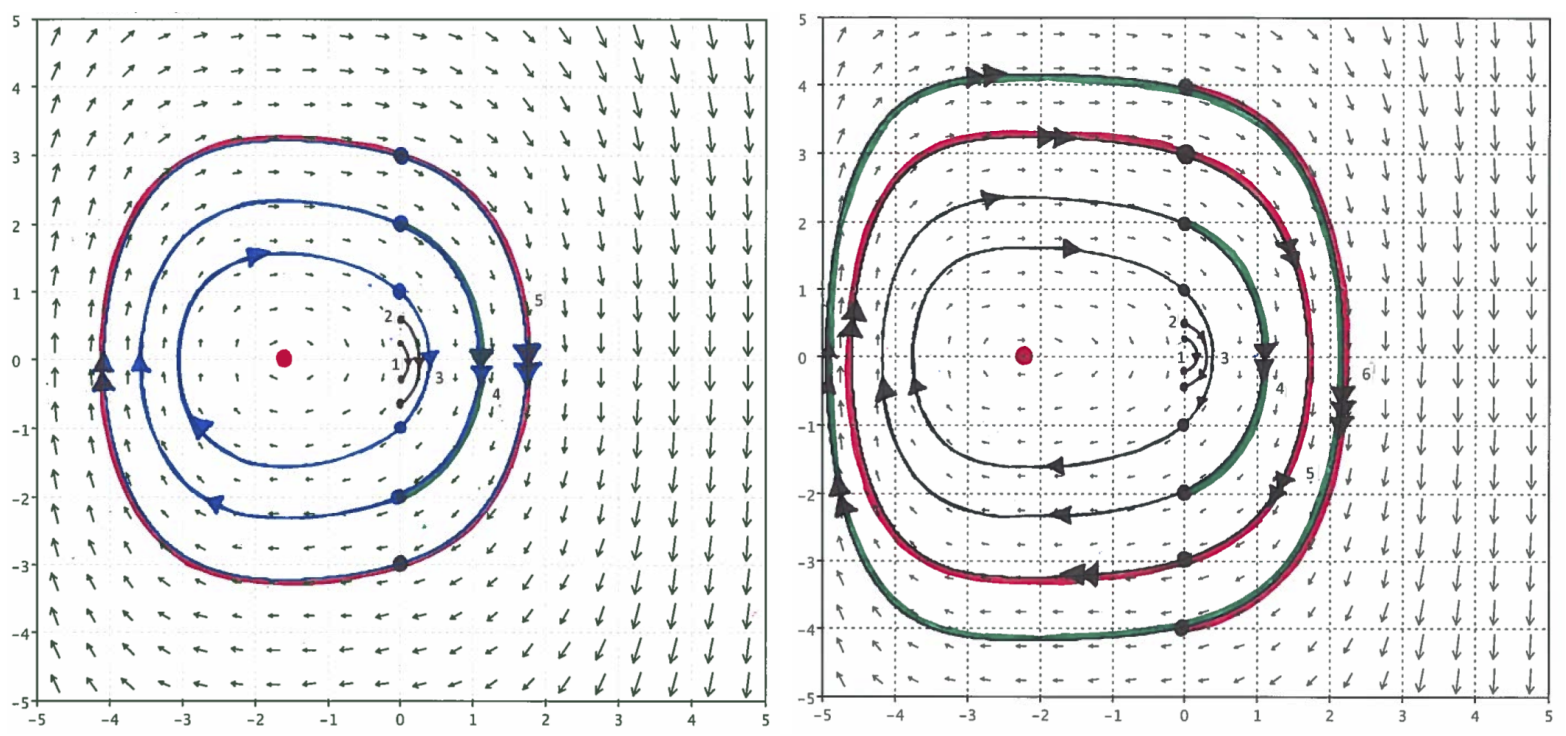

Figure 2.14: On the left, the phase plane $\left(u, u^{\prime}\right)$ for $N=3$ and $\lambda=1$ is displayed. We show five solution curves that satisfy the model and the boundary conditions start from inside to the outside numbered 1 to 5 . Initial and final values are indicated by black bullets, the red dot is the critical point of the system. The arrows provide information about the direction and the number of rotations. Solutions 3 and 5 are periodic, solution 4 is semi-periodic and solutions 1 and 2 are similar to the (lower and upper) ones as in the case $N=2$. On the right we see the phase plane $\left(u, u^{\prime}\right)$ for $N=5$ and $\lambda=1$. We show six solution curves that satisfy the model and the boundary conditions. Solutions 3 and 5 are periodic, solutions 4 and 6 are semi-periodic and solutions 1 and 2 are similar to the (lower and upper) ones as in the case $N=2$. We could construct further numerical solutions as well: there is no theoretical, only a numerical, limit.
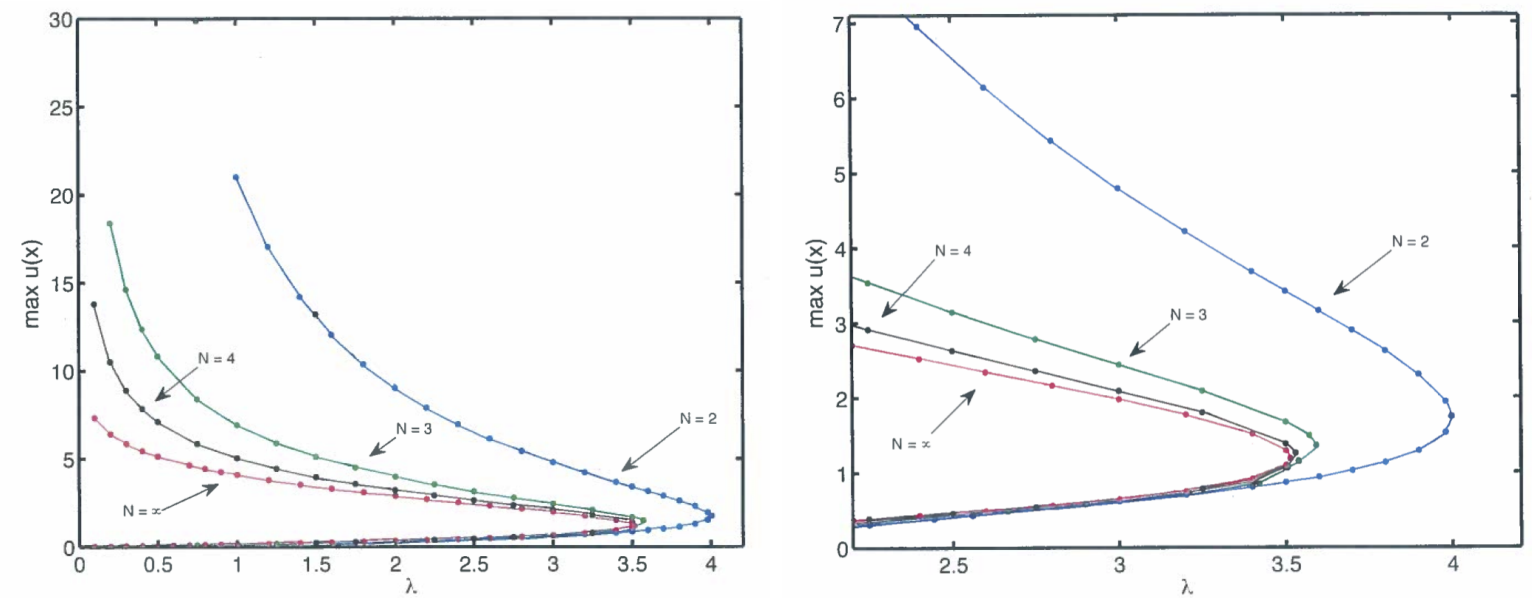

Figure 2.15: The numerical convergence of the bifurcation curves (positive solutions for all $N$ ) of model (2.1), when $N$ is approaching infinity (left) and a close up near the value $\lambda=\lambda_{c}$ (right). 


\section{Remark}

Model (2.1) is actually the classical Gelfand-Bratu problem when $N$ is approaching infinity. We discussed the behavior of the solutions of model (2.1) and other cases, which depend on the index $N$. For all $N$, we obtained a bifurcation curve for positive solutions (lower, upper and one solution). When we allow periodic and semi-periodic solutions (with possible negative values) then we obtain additional curves, as mentioned in section 2.4.3. Figure 2.15 shows the numerical convergence ( $N$ approaching infinity) of the bifurcation curves (positive solutions only) for all $N$ of model (2.1). When we take the limit $N$ approaching infinity, the bifurcation curves move towards the curve for $N=\infty$. This limit can be both uniform and non-uniform. For positive solutions, the limit is uniform for all $N$ and non-uniform for the case $N=3,5,7, \ldots$ (with periodic and semi-periodic solutions).

\subsection{Epilogue}

In this final section we summarize all our theoretical and numerical results. Figures 2.16 2.19 display all types of solutions and bifurcations connected with model (2.1) . In figure 2.16 we show the possible shapes of the periodic and semi-periodic solutions. The encircled numbers indicate the period for each case and the sign whether the first extremum in the solution is a maximum $(+)$ or a minimum (-), respectively. Figures 2.17, 2.18 and 2.19 illustrate the relation between the first extremum $M$ and the parameter $\lambda$ in model (2.1). Note that, in Figure 2.19 (the case $N \in \mathcal{N}_{3}$ ) the parameters $c, d$ and $T$ are defined in the following way. The value of $-c$ corresponds with the location of the center point in the phase plane (see also the red dot in Figure 2.14) and the value of $-d$ is the intersection of the periodic orbit going through $\left(u, u^{\prime}\right)=(0,0)$ with the $u$-axis. The period of this orbit is denoted by $T$. We observe that, for $N=1$, we obtain $c=1, d=2$ and $T=2 \pi$, as can be seen in Figure 2.18. It is also interesting to mention that the curves in Figure $2.19\left(N \in \mathcal{N}_{3}\right)$ are deformations of the ones in Figure $2.18(N=1)$. This behaviour will be theoretically explained in an upcoming article [146].

\subsection{Summary}

In this chapter, we presented numerical solutions of a truncated Bratu-Picard model by using both standard and nonstandard finite difference approximations. Solutions $u_{\lambda}^{N}(x)$ depending on the parameter $\lambda$ and $N$ were constructed by applying the nonlinear solver routine fsolve.m of Matlab. We described a series of theoretical properties of the solutions of model (2.1). For example, we analyzed the symmetry, the asymptotic behaviour and the uniqueness of the solutions for all indices $N$ and all values of the parameter $\lambda$. For the complete numerical interpretation of model (2.1) we distinguish different cases. By using the FD approximations, numerical results showed the existence of two, one and zero solutions for $\lambda>0$ and $N \in \mathcal{N}_{2}$, which resembles the $N=\infty$ case.

Investigation of the case $N \in \mathcal{N}_{3}$ indicated that we obtain infinitely many solutions, which are calculated numerically (for a large, but finite, set of solutions). These solutions are either periodic or semi-periodic which can be explained in phase plane $\left(u, u^{\prime}\right)$ diagrams. We also presented the bifurcation nature of model (2.1) for each case. We compared several finite difference schemes and we observed that with an appropriate initial guess having a suitable amplitude, the NSFD schemes provide more accurate results than the SFD scheme. We also found numerically that the NSFD approximations converge to the solutions (the lower and upper one) for each case. Further, Tables 2.7 and 2.8 summarize results on the location of the critical value $\lambda_{c}$ and on the type of solution, depending on $N$ and $\lambda$, respectively. In these graphs, the vertical axis is 


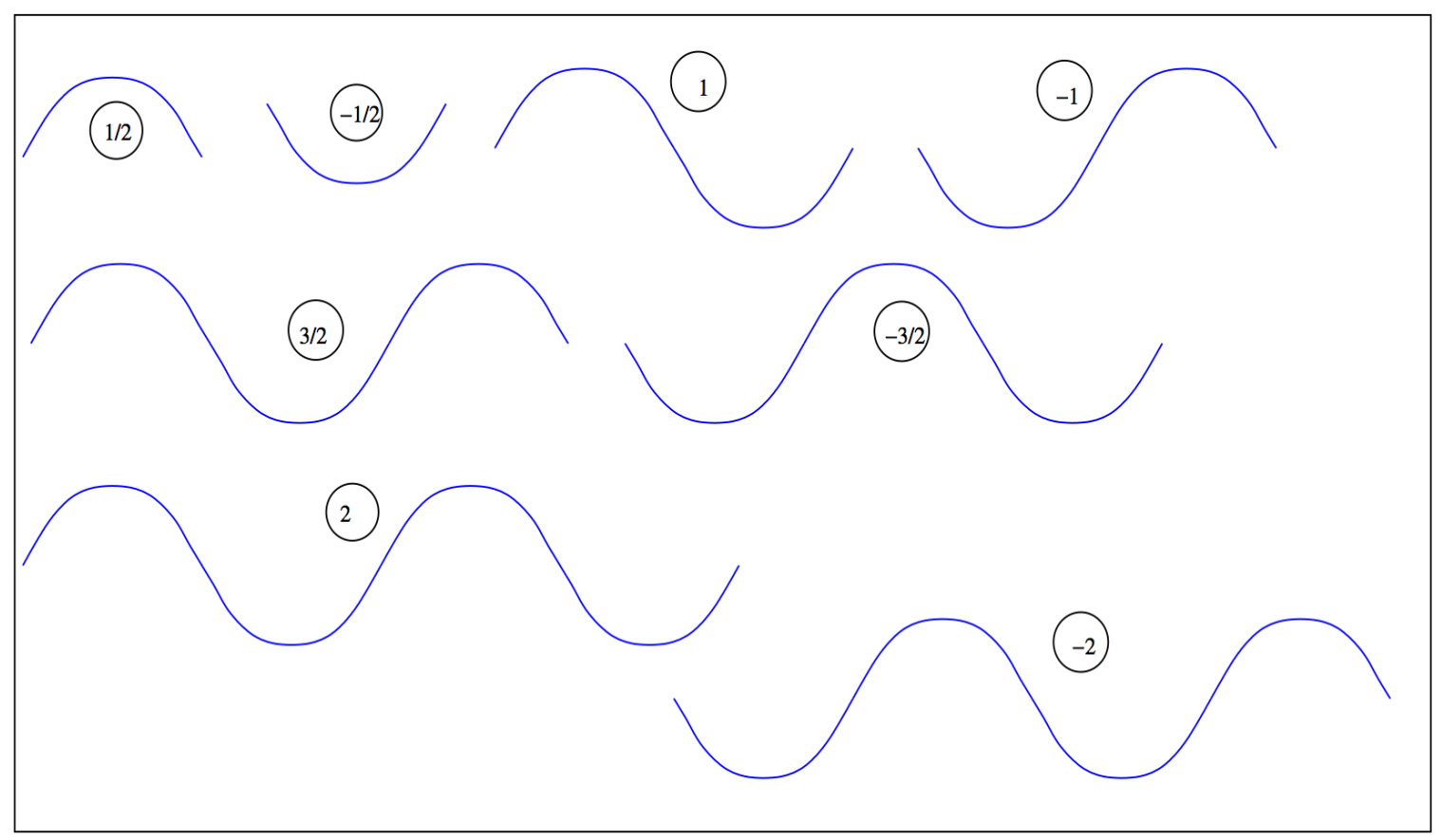

Figure 2.16: All possible types of solutions that are shown in Figures 2.17, 2.18, 2.19 can be characterized by a single number. The sign indicates the sign of the slope of the solution at $x=0$ and the number the periodicity of the solution, respectively. For example, $-3 / 2$ represents a solution with a first extremum that is negative and has one and a half period which is also denoted as 'semi-periodic' in the main text.

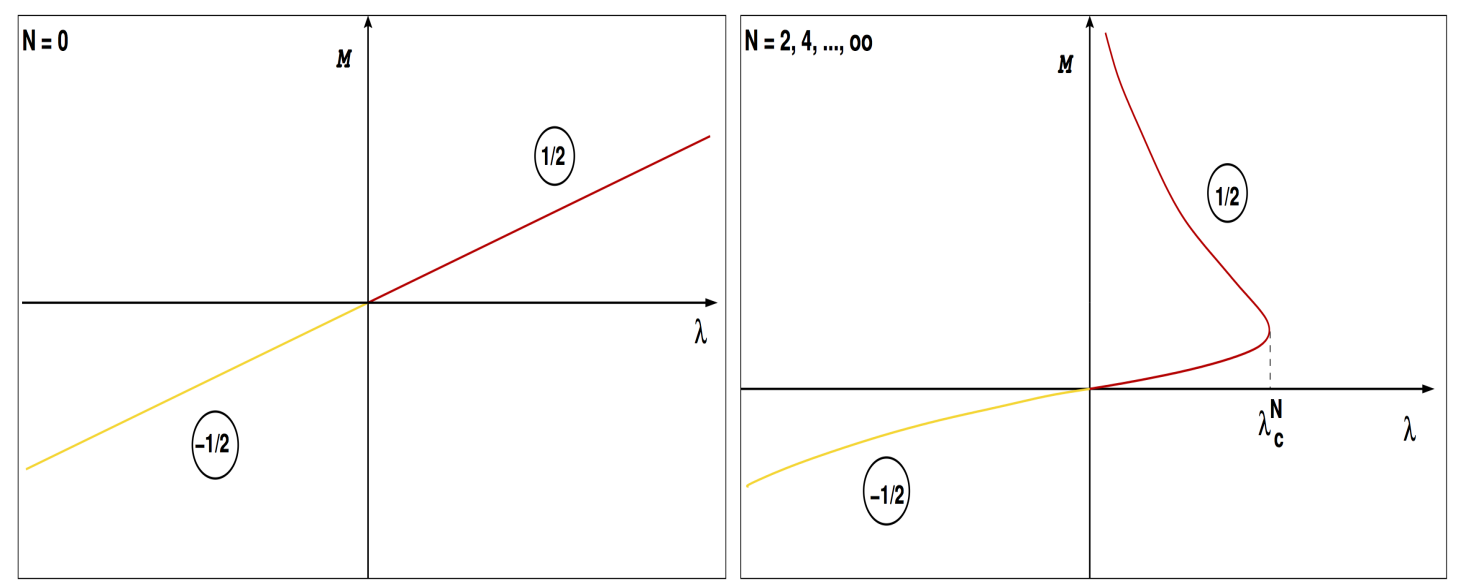

Figure 2.17: The analytical bifurcation diagram for $N=0$ (left) and $N \in \mathcal{N}_{2}$ (right). The encircled numbers are defined in Figure 2.16. The vertical axis is represented by the first extremum $M$ in the solution. 


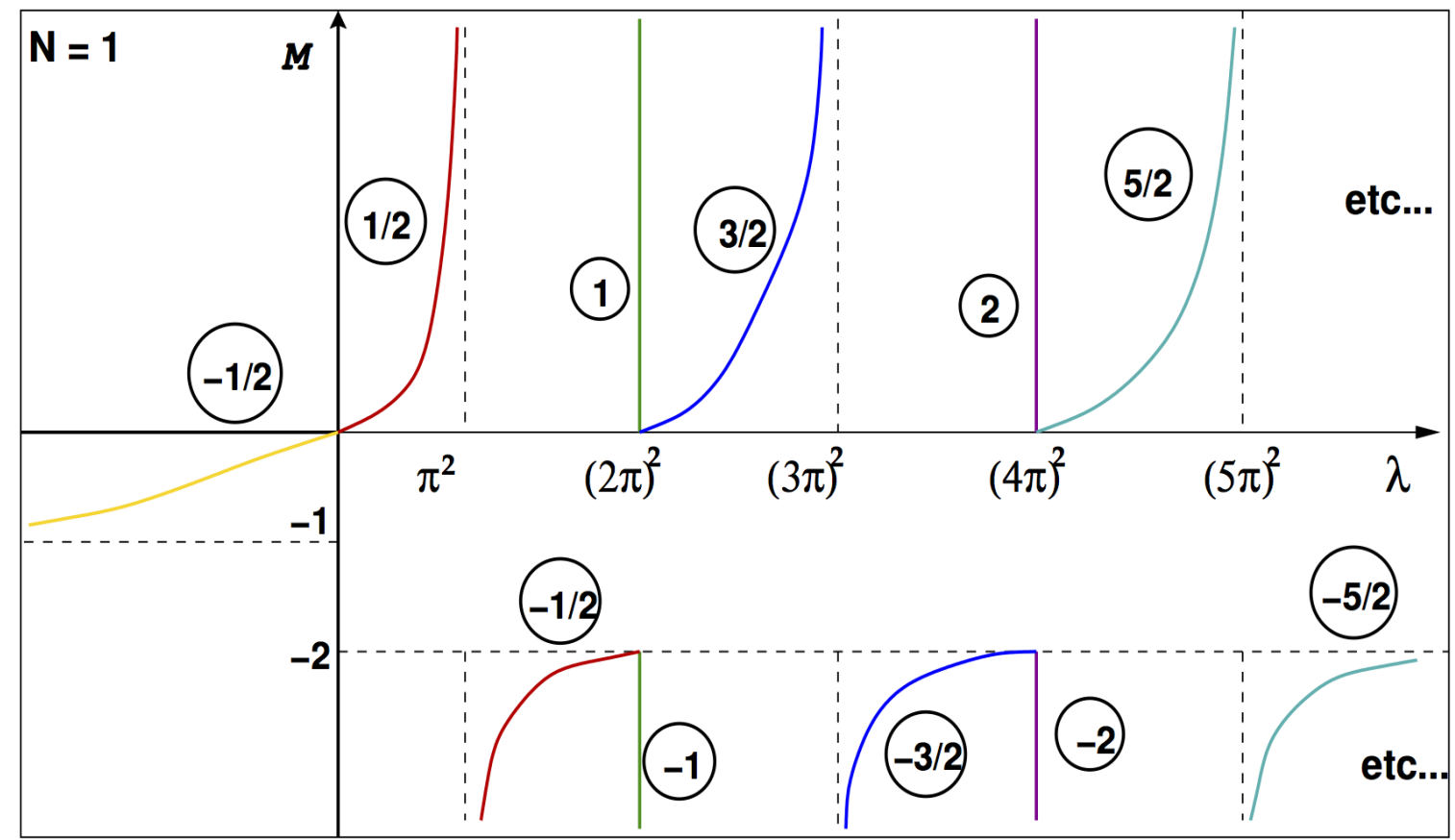

Figure 2.18: The analytical bifurcation diagram for $N=1$. The encircled numbers are defined in Figure 2.16. The vertical axis is represented by the first extremum $M$ in the solution.

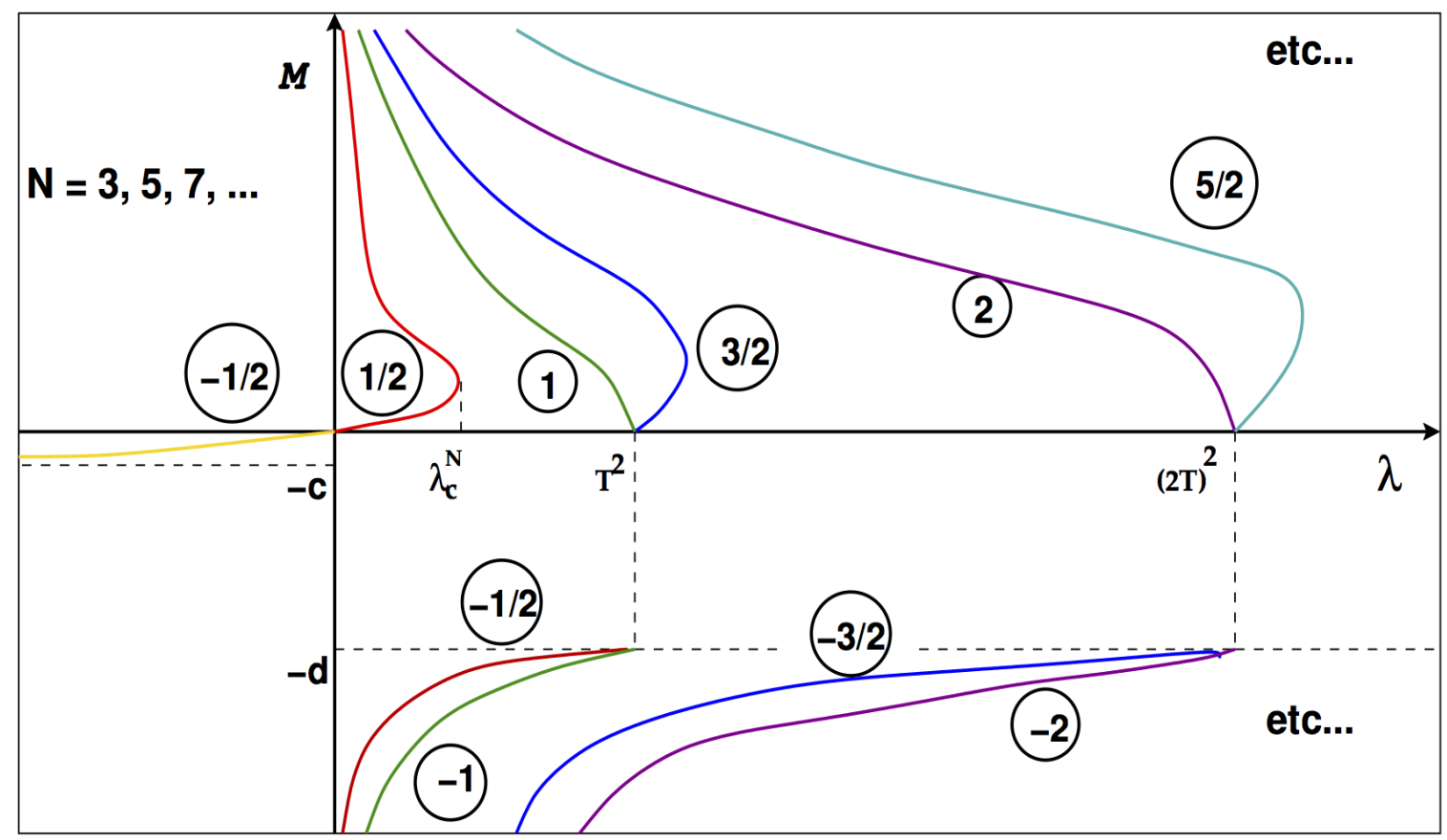

Figure 2.19: The analytical bifurcation diagram for $N \in \mathcal{N}_{3}$. The encircled numbers are defined in Figure 2.16. The vertical axis is represented by the first extremum $M$ in the solution. 
represented by the number $M$, which denotes the value of the first extremum that appears in the solution, counted from the left boundary. Finally, in the upcoming article 146, the authors report on all theoretical aspects connected with the results depicted in Figure 2.19.

Table 2.8: The dependence of the number of solutions on the index $N$ and $\lambda$. For $N=0$ there is a unique solution of (2.1), for $N=1$ we have a unique solution for $\lambda \in \mathbb{R}$ (except for $\lambda=4 \pi^{2}, 9 \pi^{2}, \ldots$ for which there are infinitely many solutions), for $N \in \mathcal{N}_{2}$ the number of solutions of equation (2.1) can be zero (for $\lambda>\lambda_{c}$ ), one (for $\lambda \leq 0$ and for $\lambda=\lambda_{c}$ ) or two (for $0<\lambda<\lambda_{c}$ ) and for $N \in \mathcal{N}_{3}$ only a unique solution exists for $\lambda \leq 0$, respectively. In the latter case, we obtain infinitely many solutions of (2.1) for $\lambda>0$.

\begin{tabular}{|c|c|c|c|c|c|c|c|c|c|c|}
\hline \hline$N$ & 0 & 1 & 2 & 3 & 4 & 5 & 6 & 7 & $\ldots$ & $\infty$ \\
\hline$\#$ solutions & 1 & $1, \infty$ & $0,1,2$ & $1, \infty$ & $0,1,2$ & $1, \infty$ & $0,1,2$ & $1, \infty$ & $\ldots$ & $0,1,2$ \\
\hline
\end{tabular}





\section{Chapter 3}

\section{Higher order non-uniform grids for singularly perturbed convection-diffusion-reaction problems}

\section{Introduction}

It is well known that solutions of boundary value problems for differential equations with small perturbation parameters having higher derivatives may exhibit a boundary-layer behavior [6], [55], [135]. This type of BVPs has many applications in mathematical modelling of physical and engineering problems, such as fluid dynamics at high Reynolds numbers [70], [4], [33], aerodynamics [117], mass heat transfer [116], atmospheric models [121] and reaction-diffusion processes [70].

Nowadays boundary layer problems are addressed mainly with numerical techniques. A large number of numerical techniques have been proposed by various authors for singularly perturbed boundary value problem (SPBVPs). Finite difference approximations on non-uniform grids have been investigated in [134], [125] and [54]. Numerical approximations and solutions for SPBVPs have been discussed in [5] and [109]. Theory and applications of singular perturbations in boundary-layer problems and multiple timescale dynamics have been reported in [135]. To deal with the steep solution regions, two different forms of computational non-uniform grids by dividing the region into two or more parts with different uniform spacing were discussed in [113] for boundary-layer problems.

Due to the presence of boundary layers the numerical treatment of SPBVPs has computational difficulties. Central finite difference schemes on uniformly distributed grids are usually applied to solve SPBVPs numerically. However, in many cases, unphysical oscillations are observed in the numerical solutions [5], [6]. Finite difference schemes on uniform grids are relatively simple, but they are not accurate and efficient for problems with boundary layers, because of the potential appearance of numerical oscillations. If the number of points is not large enough to resolve the boundary layer, the numerical solution is likely to have huge errors. The use of a large number of grid points to resolve this problem makes the total computation time unacceptably large. To eliminate the oscillations in the solutions, one needs a very fine grid at the layer

\footnotetext{
The main part of this chapter is based on the article published as [57]: S. Iqbal and P. A. Zegeling, Journal of Computational Mathematics and Mathematical Physics, Springer, 59 (12): 2057-2079, 2019. Section 3.5 is based on the article published as [58]: S. Iqbal and P. A. Zegeling, Numerical Geometry, Grid Generation and Scientific Computing, Lecture Notes in Computational Science and Engineering, Springer, 131: 109-124, 2019.
} 
regions. This may be improved either by using non-uniform grids (prescribed) or by an adaptive grid (generated by an additional equation). Finite difference approximations on non-uniform grids have been discussed in [134], [125], [5], [135], [109] for SPBVPs. At the beginning of the 90's, special piecewise uniform grids have been introduced by Shishkin [93], in which simple structured grids can be used for the numerical approximation of SPBVPs. All these numerical approaches on non-uniform grids are more accurate than for the uniform case but with the same order of convergence. A truncation error analysis introduced by the use of non-uniform grids and stretched coordinates for the numerical study of the boundary-layer problems has been reported in [30], [54], [72], [136] in which numerical results are compared with those obtained on uniform grids. In [79], [29], [101] a stretched grid method was introduced in which the given boundarylayer problems are transformed into new coordinates by a smooth mapping which concentrates the grid points in the steep regions without an increase of the total number of grid points. This concentration improved the spatial resolution in the region of large variation which enhanced the accuracy in numerical solutions.

Adaptive grid methods, graded grid difference schemes and uniformly accurate finite difference approximations for SPBVPs have been presented in [31], [40] and [12], respectively. The rate of convergence of finite difference schemes on non-uniform grids and superconvergent grids for two point BVPs have been described in [56], [143] and [26]. Further, finite difference approximations of multidimensional steady and unsteady convection-diffusion-reaction problems have been discussed in [68], [69] and [28]. All these numerical approaches on non-uniform grids are more accurate than for the uniform case but with the same order of convergence. The supraconvergence phenomenon of the central finite difference scheme is well-known and it was studied rigorously. In literature, the increase from second to higher order accuracy for linear boundary value problems has been reported in [19] and recently in [71]. Second order central finite differences on non-uniform grids are discussed in [71], in which an adaptive numerical method is applied to obtain 4th-order of convergence.

In the present study, we first focus on special central finite difference approximations on an optimal choice of the non-uniform grid which demonstrate not only higher order of convergence (from $2^{\text {nd }}$ order to $4^{\text {th }}$ order) but also an increase in the accuracy for nonlinear SPBVPs. We present two theoretical properties of the solution of the SPBVPs, which exhibit boundary layer phenomena. The main contribution in this chapter is first to construct the exact optimal higher order non-uniform grid transformations based on truncation error analysis. Furthermore, we use these (optimal) grid transformations to compute the numerical solution of nonlinear SPBVPs. In the second part, the goal is to propose an efficient adaptive numerical method which can solve approximately such SPBVPs with an accuracy independent of the value of the perturbation parameter. We extend the results from $4^{\text {th }}$ to $6^{\text {th }}$ order approximations not only for linear BVPs, but we are also able to obtain an (almost) $6^{\text {th }}$ order accuracy for nonlinear models. For this, we propose a non-uniform equidistributed grid and show that the second order central finite difference scheme is substantially upgraded to $6^{\text {th }}$ order on this refined grid. This supra-convergence is obtained by using an appropriate monitor function, which depends on the lowest derivative. For numerical illustration, we choose nonlinear convection dominated singularly perturbed convection-diffusion-reaction problems which exhibit boundary layer phenomena. Numerical results demonstrate the effectiveness of the proposed methods for obtaining higher order of convergence.

The present chapter is organized as follows: a convection dominated SPBVP is presented in section 3.1. Theoretical observations of the considered SPBVP are presented in section 3.2. Finite difference approximations on uniform grids are described in section 3.3. In section 3.4, we develop the higher order non-uniform grid transformation. The general central finite difference scheme on non-uniform grids and the coordinate transformation for the SPBVPs are provided 
Higher order non-uniform grids for singularly perturbed convection-diffusion-reaction problems

in sections 3.4 .1 and 3.4.2, respectively. In section 3.4.3, a local truncation error analysis of the discretized system to construct the $4^{\text {th }}$ order non-uniform grids is explained. To establish the adaptive $6^{\text {th }}$ order non-uniform grid, the equidistribution principle is presented in section 3.5.1. Furthermore, in section 3.5.2, numerical results for different choices of non-uniform grids, are discussed. Finally, we summarize our results in section 5.6 .

\subsection{Convection-diffusion-reaction model}

Consider the following convection dominated singularly perturbed boundary value problem (SPBVP):

$$
\left\{\begin{array}{l}
\epsilon u^{\prime \prime}(x)+[g(u(x))]^{\prime}+f(u(x))=0, x \in[0,1], 0<\epsilon<1, \\
u(0)=\alpha \quad u(1)=\beta
\end{array}\right.
$$

with a small positive perturbation parameter $\epsilon$ and parameters $\alpha, \beta \geq 0$. We assume that there exists a unique solution for model (3.1). Note, however, that multiple solutions may exist for specific choices of the functions $f$ and $g$ (see Chapter 2, [148] and [7]). Furthermore, we may expect boundary layers in the solution as well. For $g^{\prime}(u)>0$ and $0<\epsilon \ll 1$, problem (3.1) has a unique solution and exhibits a boundary layer of $\mathcal{O}(\epsilon)$ near $x=0$, whereas, for $f(u)=0$ and $g^{\prime}(u)<0$, this occurs at $x=1$.

\subsection{Theoretical observations}

In this section, we would like to present two new theoretical results connected with a generalized version of model (3.1):

$$
\left\{\begin{array}{l}
\epsilon\left[D(u(x)) u^{\prime}(x)\right]^{\prime}+[g(u(x))]^{\prime}+f(u(x))=0, x \in\left[x_{L}, x_{R}\right], 0<\epsilon \ll 1, \\
u\left(x_{L}\right)=\alpha \quad u\left(x_{R}\right)=\beta .
\end{array}\right.
$$

where, additionally, we assume that $D(u)>0, D^{\prime}(u) \geq 0$ and $g^{\prime}(u)>0$. The results are generalizations of the ones obtained in [7]. Therein, the authors consider the special case $D=1$, $g(u)=2 u$ and $f(u)=e^{u}$.

\subsubsection{Theorem 1}

For the choice $\alpha=\beta \geq 0$ and $f(u)>0$, the solution $u(x)$ of model (3.2), assuming it exists, has a unique maximum (no minimum).

Proof: Suppose first that $u$ is constant, i.e., $u(x)=\alpha, \forall x \in\left[x_{L}, x_{R}\right]$. This solution satisfies the boundary conditions, but not differential equation (3.2), since $f(u(x))=f(\alpha)>0$. Therefore, $u(x)$ must have interior extreme values. At such an extremum, say at $x=\gamma$, we have $u^{\prime}(\gamma)=0$ and, using the chain rule, we find

$$
\epsilon D^{\prime}(u(\gamma)) u^{\prime}(\gamma)+\epsilon D(u(\gamma)) u^{\prime \prime}(\gamma)+g^{\prime}(u(\gamma)) u^{\prime}(\gamma)+f(u(\gamma))=0
$$

From this follows that

$$
\epsilon D(u(\gamma)) u^{\prime \prime}(\gamma)=-f(u(\gamma))<0
$$

As a consequence, the curvature of the solution is negative and, at $x=\gamma$, we find a maximum value. There can not be further maxima, since that would imply the existence of at least one 
minimum, which is not possible with $u^{\prime \prime}(\gamma)<0$. Conclusion: there is only one maximum.

\subsubsection{Theorem 2}

Assume that $g^{\prime}(u)>0$. For $\epsilon \downarrow 0$, we find the following asymptotic expression for the solution derivative at the right boundary point of model (3.2):

$$
u^{\prime}\left(x_{R}\right)=-\frac{f(\beta)}{g^{\prime}(\beta)}-\epsilon \frac{f(\beta)}{\left[g^{\prime}(\beta)\right]^{4}}\left\{D^{\prime}(\beta)[f(\beta)]^{2} g^{\prime}(\beta)+D(\beta)\left[f(\beta) g^{\prime \prime}(\beta)+f^{\prime}(\beta) g^{\prime}(\beta)\right]\right\}+\mathcal{O}\left(\epsilon^{2}\right) .
$$

Proof: Since we are only interested in the solution near $x=x_{R}$, i.e., away from the boundary layer near $x=x_{L}$, we may use the regular expansion: $u(x)=u_{0}(x)+\epsilon u_{1}(x)+\mathcal{O}\left(\epsilon^{2}\right)$. Applying the expansion $f(u)=f\left(u_{0}\right)+\epsilon u_{1}(x) f^{\prime}\left(u_{0}\right)+\mathcal{O}\left(\epsilon^{2}\right)$, and similar ones for $f^{\prime}(u), g(u), D(u), D^{\prime}(u)$ and $g^{\prime}(u)$, respectively, and collecting terms of equal power in $\epsilon$, we arrive at

$$
\begin{aligned}
& \epsilon^{0}: \quad g^{\prime}\left(u_{0}\right) u_{0}^{\prime}+f\left(u_{0}\right)=0, \quad u_{0}\left(x_{R}\right)=\beta, \\
& \epsilon^{1}: \quad D^{\prime}\left(u_{0}\right)\left[u_{0}^{\prime}\right]^{2}+D\left(u_{0}\right) u_{0}^{\prime \prime}+g^{\prime}\left(u_{0}\right) u_{1}^{\prime}+u_{1} g^{\prime \prime}\left(u_{0}\right) u_{0}^{\prime}+u_{1} f^{\prime}\left(u_{0}\right)=0, \quad u_{1}\left(x_{R}\right)=0, \\
& \text { etceter } \ldots
\end{aligned}
$$

From the first equation immediately follows

$$
u_{0}^{\prime}\left(x_{R}\right)=-\frac{f(\beta)}{g^{\prime}(\beta)},
$$

which gives the first term of the theorem. Using this result, one quotient rule and the fact that $u_{0}\left(x_{R}\right)=\beta$ and $u_{1}\left(x_{R}\right)=0$, we can derive

$$
u_{0}^{\prime \prime}\left(x_{R}\right)=-\frac{f^{\prime}(\beta) g^{\prime}(\beta)-f(\beta) g^{\prime \prime}(\beta)}{\left[g^{\prime}(\beta)^{2}\right]} u_{0}^{\prime}\left(x_{R}\right) .
$$

Substituting this expression in the second equation, gives the second term

$$
u_{1}^{\prime}\left(x_{R}\right)=-\frac{f(\beta)}{\left[g^{\prime}(\beta)\right]^{4}}\left\{D^{\prime}(\beta)[f(\beta)]^{2} g^{\prime}(\beta)+D(\beta)\left[f(\beta) g^{\prime \prime}(\beta)+f^{\prime}(\beta) g^{\prime}(\beta)\right]\right\} .
$$

Next, we note that the equation has a stable slow manifold $M$ with an $\mathcal{O}\left(\epsilon^{2}\right)$ approximation by the manifold $M_{0}$ described by $u_{0}(x)+\epsilon u_{1}(x)$. This observation can be found by applying Fenichel's geometric perturbation theory (for more details, see [7, 135]). This implies that, away from the boundary layer at $x=x_{L}$, we find near $x=x_{R}$ :

$$
u(x)=u_{0}(x)+\epsilon u_{1}(x)+\mathcal{O}\left(\epsilon^{2}\right), \text { and } u^{\prime}(x)=u_{0}^{\prime}(x)+\epsilon u_{1}^{\prime}(x)+\mathcal{O}\left(\epsilon^{2}\right) .
$$

When choosing $x=x_{R}$, the results follows.

In Table 3.1 we illustrate this for the special case with $\alpha=\beta=0, x_{L}=0, x_{R}=1, f(u)=e^{u}$, $g(u)=2 u$ and $D=1$ (as in [7]). Then the result in Theorem 2 predicts that $u_{0}^{\prime}(1)=-1 / 2$ and $u_{1}^{\prime}(1)=-1 / 8$ and $u^{\prime}(1)=-1 / 2-\epsilon / 8+\mathcal{O}\left(\epsilon^{2}\right)$. Table 3.1 confirms this asymptotic behavior. 
Higher order non-uniform grids for singularly perturbed convection-diffusion-reaction problems

Table 3.1: Asymptotic results for special choices of the functions and parameters in Theorem 2.

\begin{tabular}{|c||c||c||c|}
\hline \hline$\epsilon$ & $u^{\prime}(1)$ & $\left|u^{\prime}(1)-\left(-\frac{1}{2}\right)\right|$ & $\left|u^{\prime}(1)-\left(-\frac{1}{2}-\frac{\epsilon}{8}\right)\right|$ \\
\hline \hline 0.5 & -0.5414203 & 0.0414 & 0.0211 \\
0.05 & -0.5065322 & 0.0065 & $2.7211 \mathrm{e}-04$ \\
0.005 & -0.5003230 & $3.2300 \mathrm{e}-04$ & $1.4102 \mathrm{e}-05$ \\
\hline
\end{tabular}

\subsection{Discretizations on uniform grids}

To compute numerical solutions of (3.1) we first work out a standard finite difference scheme by discretizing the differential equation and boundary conditions. Subdivide the continuous spatial interval $I=[0,1]$ into a uniformly distributed discrete set of grid points $\left\{x_{j}\right\}_{j=0}^{J}$, i.e., $x_{j}=j h, \quad j=0,1, \ldots, J$ with grid size $h=\frac{1}{J}$. With $u_{j} \approx u\left(x_{j}\right)$, a three-point central finite difference discretization of (3.1) is then given by

$$
\epsilon \frac{u_{j+1}-2 u_{j}+u_{j-1}}{h^{2}}+\frac{g\left(u_{j+1}\right)-g\left(u_{j-1}\right)}{2 h}+f\left(u_{j}\right)=0, \quad j=1, \ldots, J-1
$$

with boundary conditions $u_{0}=0, u_{J}=1$. This scheme has second order accuracy:

$$
\tau=-\frac{h^{2}}{2} u_{j}^{\prime \prime \prime \prime}+\mathcal{O}\left(h^{4}\right) \text {. }
$$

This may cause the standard uniform-grid technique to be computationally inefficient, in situations where the solution possesses steep parts. In those cases, to obtain an accurate numerical approximation, a very large number of grid points should be used (or, similarly, a very small $h$ ). To clarify this phenomenon, we consider $f(u)=0$ and $g(u)=u$ in model (3.1). Then the finite difference approximation (3.3) becomes

$$
\epsilon \frac{u_{j+1}-2 u_{j}+u_{j-1}}{h^{2}}+\frac{u_{j+1}-u_{j-1}}{2 h}=0, \quad j=1, \ldots, J-1 .
$$

An exact numerical solution of (3.5) can be found [109] and reads

$$
u_{j}=\frac{\left(\frac{1+P_{e}}{1-P_{e}}\right)^{j}-1}{\left(\frac{1+P_{e}}{1-P_{e}}\right)^{J}-1}, \quad j=1, \ldots, J-1,
$$

where $P_{e}:=\frac{h}{2 \epsilon}$ denotes the mesh-Peclet number. It can easily be checked that, for $J<\frac{1}{2 \epsilon}$, i.e., $P_{e}>1$, the numerical solution exhibits an unwanted oscillatory behavior (see also Figure 3.1), whereas, for $J>\frac{1}{2 \epsilon}$, i.e., $0<P_{e}<1$, monotone numerical solutions are obtained. In the case that $0<\epsilon \ll 1$, this means that we would need $J \gg 1$ (or $h \ll 1$ ) to satisfy the monotonicity conditions. It is obvious that this leads to an inefficient numerical process. Another straightforward discretization is given by an upwind approximation of the first derivative in (3.1) with $g(u)=u$ and $f(u)=0$ :

$$
\epsilon \frac{u_{j+1}-2 u_{j}+u_{j-1}}{h^{2}}+\frac{u_{j}-u_{j-1}}{h}=0, \quad j=1, \ldots, J-1 .
$$

Again, we can find an exact numerical solution [109] of this system:

$$
u_{j}=\frac{\left(1+P_{e}\right)^{j}-1}{\left(1-P_{e}\right)^{J}-1}, \quad j=1, \ldots, J-1 .
$$

For this method, we always have $1+P_{\rho}>1$ and, therefore, obtain a monotone solution, independent of the value of $P_{e}$ (see Figure 3.1). However, the error now is reduced to first order, i.e., $\mathcal{O}(h)$. 

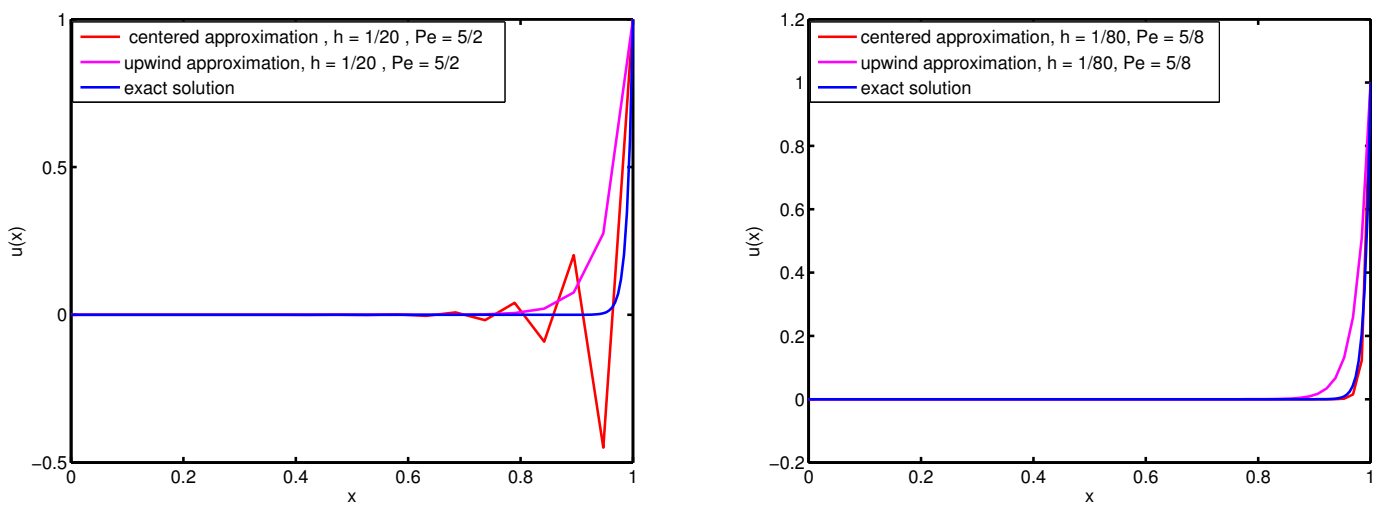

Figure 3.1: Behaviour of the exact numerical solutions (3.5) and (3.6), depending on the parameter $P_{e}$, for the case $f(u)=0$ and $g(u)=u$. We show the oscillatory behaviour when $P_{e}>1$ (left panel) and smooth solutions when $0<P_{e}<1$ (right panel).

\subsection{Fourth-order non-uniform grid generation}

To generate the non-uniform grid for model (3.1), we follow the following steps:

Step 1: Discretize problem (3.1) on a non-uniform grid.

Step 2: Transform the discretized system from the physical coordinate $x$ to a computational coordinate $\xi$.

Step 3: Construct the optimal non-uniform grid transformation $x(\xi)$ based on a local truncation error analysis of the discretized system.

Step 4: Compute the solution of the discretized system of problem (3.1) on the optimal non-uniform grid.

\subsubsection{Discretizations on non-uniform grids}

In order to deal with the appearance of steep boundary layers in the given model (3.1), nonuniformly distributed grids could be used to obtain more efficient and more accurate numerical solutions. An example of a non-uniform discretization of the first derivative in (3.1) is given by

$$
u_{j}^{\prime} \approx \frac{u_{j+1}-u_{j-1}}{2 h_{j}} .
$$

Similarly, the second derivative can be approximated by

$$
u_{j}^{\prime \prime} \approx \frac{\frac{u_{j+1}-u_{j}}{p}-\frac{u_{j}-u_{j-1}}{q}}{h_{j}},
$$

where the $h_{j}$ 's are computed as:

$$
p:=x_{j+1}-x_{j}, \quad q:=x_{j}-x_{j-1}, \quad h_{j}:=\frac{p+q}{2} .
$$

Approximating the derivatives in model (3.1) by using these expressions yields the following numerical approximation:

$$
\epsilon \frac{\frac{u_{j+1}-u_{j}}{p}-\frac{u_{j}-u_{j-1}}{q}}{h_{j}}+\frac{g\left(u_{j+1}\right)-g\left(u_{j-1}\right)}{2 h_{j}}+f\left(u_{j}\right)=0, \quad j=1,2, \ldots, J-1
$$


Higher order non-uniform grids for singularly perturbed convection-diffusion-reaction problems

with boundary conditions $u_{0}=\alpha, u_{J}=\beta$. The idea is to choose a non-uniform central finite difference method such that the steep parts in the solution can be resolved. We will do this by performing the following steps. First, we transform the original model from the physical domain $I=[0,1]$ to a computational domain $I^{*}$.

\subsubsection{Coordinate transformation}

Let $x$ and $\xi$ denote the physical and computational coordinates, respectively. Without loss of generality, we define a composed function $v(\xi):=(u \circ x)(\xi)=u(x(\xi))$ and a coordinate transformation between $x$ and $\xi$ as:

$$
x=x(\xi), \quad \xi \in I^{*}=[0,1],
$$

where $x(0)=0$ and $x(1)=1$. The computational domain $I^{*}$ can be discretized into $J$ equal segments $\left\{\xi_{j}=j \Delta \xi\right\}_{j=0}^{J}$ with $\Delta \xi=\frac{1}{J}$. The first derivative is then transformed as

$$
u^{\prime}=\frac{d u}{d x}=\frac{d v}{d \xi} \frac{d \xi}{d x}=\frac{d v}{d \xi} \frac{1}{x_{\xi}}
$$

where $x_{\xi}=\frac{d x}{d \xi}$. The SPBVP (3.1) on the computational domain $I^{*}$ can be written as

$$
\frac{\epsilon}{x_{\xi}} \frac{d}{d \xi}\left(\frac{1}{x_{\xi}} \frac{d v}{d \xi}\right)+\frac{1}{x_{\xi}} \frac{d}{d \xi}(g(v(\xi)))+f(v(\xi))=0, \quad v(0)=\alpha, \quad v(1)=\beta .
$$

The general idea behind this is to transform the, potentially, steep solution $u(x)$ in the $x$ coordinate to the, intended, milder solution $v(\xi)$ in the $\xi$-coordinate (see Figure 3.2). We also
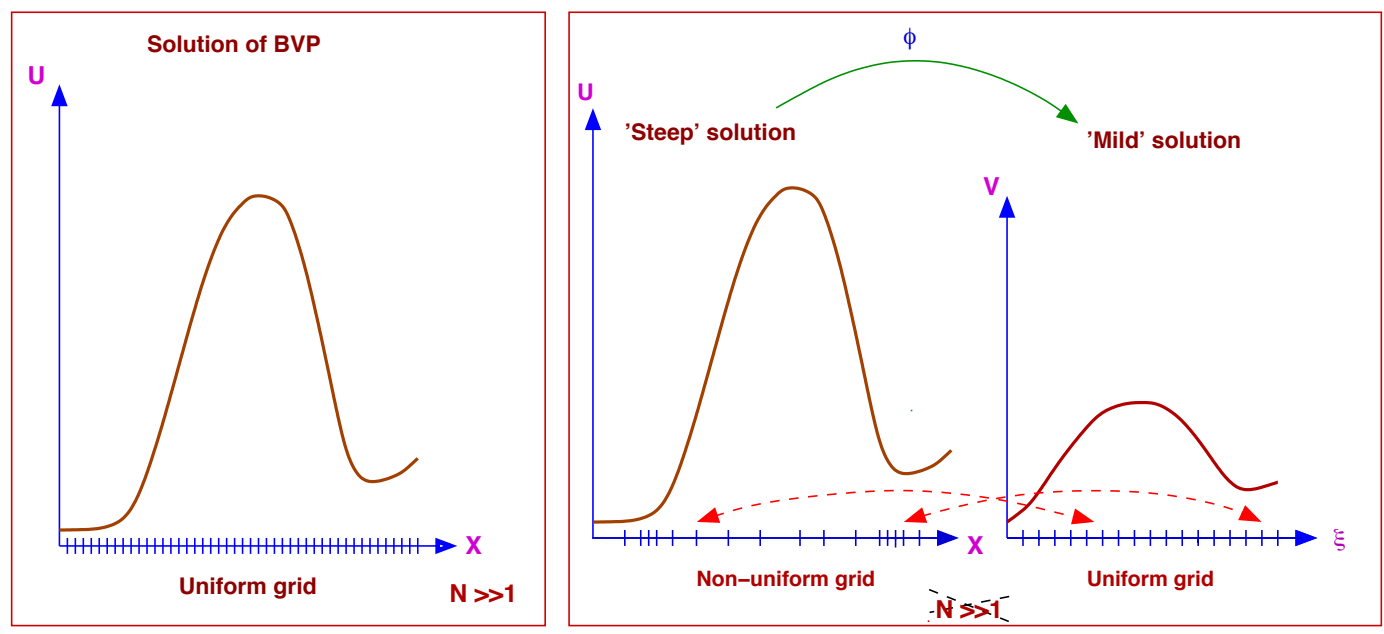

Figure 3.2: A transformation $\phi$ from a uniform to a non-uniform grid in which the steep solution $u(x)$ in the $x$-coordinate transforms to a milder solution $v(\xi)$ in the $\xi$-coordinate. In this figure $N$ is the number of grid points (called $J$ in the text).

assume that the Jacobian $\mathbb{J}$ of mapping $x(\xi)$ is bounded from below and above by some positive constant: $0<\mathbb{J}:=\frac{d x}{d \xi}<\infty$. We get the following from equation (3.9):

$$
\frac{\epsilon}{\mathbb{J}} \frac{d}{d \xi}\left(\frac{1}{\mathbb{J}} \frac{d v}{d \xi}\right)+\frac{1}{\mathbb{J}} \frac{d}{d \xi}(g(v(\xi)))+f(v(\xi))=0 .
$$


with the same boundary conditions $v(0)=\alpha, v(1)=\beta$. Equation 3.10 can be discretized on the uniform grid $h$ as follows:

$$
\frac{\epsilon}{\mathbb{J}_{j}}\left(\frac{v_{j+1}-v_{j}}{\mathbb{J}_{j+\frac{1}{2}}}-\frac{v_{j}-v_{j-1}}{\mathbb{J}_{j-\frac{1}{2}}}\right)+\frac{g\left(v_{j+1}\right)-g\left(v_{j-1}\right)}{2 \mathbb{J}_{j}}+f\left(v_{j}\right)=0, \quad v_{0}=\alpha, v_{J}=\beta .
$$

where the Jacobian $\mathbb{J}$ has been approximated by

$$
\mathbb{J}_{j+\frac{1}{2}}:=\frac{p}{\Delta \xi}, \quad \mathbb{J}_{j-\frac{1}{2}}:=\frac{q}{\Delta \xi}, \quad \mathbb{J}_{j}:=\frac{\mathbb{J}_{j+\frac{1}{2}}+\mathbb{J}_{j-\frac{1}{2}}}{2 \Delta \xi} .
$$

The last scheme (3.11) can be equivalently rewritten as (3.7).

\subsubsection{Local truncation error analysis}

In the following, we study some approximation properties of the scheme (3.7) on non-uniform grids. We set

$$
\varphi_{j}:=\frac{\epsilon}{h_{j}}\left[\frac{u_{j+1}-u_{j}}{p}-\frac{u_{j}-u_{j-1}}{q}\right]+\frac{g\left(u_{j+1}\right)-g\left(u_{j-1}\right)}{2 h_{j}}+f\left(u_{j}\right)=0 .
$$

and compute the consistency error of the approximation $\varphi_{j}$. For this, we need to work out Taylor expansions and compose the finite differences which appear in (3.7):

$$
\begin{aligned}
& \frac{u_{j+1}-u_{j}}{p}=u^{\prime}+\frac{p}{2 !} u^{\prime \prime}+\frac{p^{2}}{3 !} u^{\prime \prime \prime}+\frac{p^{3}}{4 !} u^{(4)}+\frac{p^{4}}{5 !} u^{(5)}+\mathcal{O}\left(p^{5}\right), \\
& \frac{u_{j}-u_{j-1}}{q}=u^{\prime}+\frac{q}{2} u^{\prime \prime}+\frac{q^{2}}{3 !} u^{\prime \prime \prime}+\frac{q^{3}}{4 !} u^{(4)}+\frac{q^{4}}{5 !} u^{(5)}+\mathcal{O}\left(q^{5}\right) .
\end{aligned}
$$

Assume further that the mapping $x(\xi)$, introduced in the previous section, is sufficiently smooth. We define $p$ and $q$ in (3.13) and (3.14) as:

$$
\begin{aligned}
& p:=x_{j+1}-x_{j}=\Delta \xi x_{\xi}+\frac{\Delta \xi^{2}}{2 !} x_{\xi \xi}+\frac{\Delta \xi^{3}}{3 !} x_{\xi \xi \xi}+\mathcal{O}\left(\Delta \xi^{4}\right), \\
& q:=x_{j}-x_{j-1}=\Delta \xi x_{\xi}-\frac{\Delta \xi^{2}}{2 !} x_{\xi \xi}+\frac{\Delta \xi^{3}}{3 !} x_{\xi \xi \xi}+\mathcal{O}\left(\Delta \xi^{4}\right) .
\end{aligned}
$$

Note that we write $x_{\xi}$ instead of $x_{\xi, j}$. The expression for the non-uniform second derivative approximation becomes:

$$
\underbrace{\frac{1}{h_{j}} \frac{u_{j+1}-u_{j}}{p}-\frac{u_{j}-u_{j-1}}{q}}_{\widetilde{u^{\prime \prime}}}=u^{\prime \prime}+\frac{p-q}{3} u^{\prime \prime \prime}+\left(\frac{\left(p^{2}-p q+q^{2}\right.}{60}\right) u^{(4)}+\mathcal{O}\left(\Delta \xi^{4}\right) .
$$

In the remainder of the text, when there is no confusion, we write for notational reasons $u^{\prime}$ instead of $u_{j}^{\prime}, u^{\prime \prime}$ instead of $u_{j}^{\prime \prime}$, etcetera. By substituting $p$ and $q$ from (3.15) into (3.16), one finds

$$
\widetilde{u^{\prime \prime}}=u^{\prime \prime}+\frac{\Delta \xi^{2}}{3} x_{\xi \xi} u^{\prime \prime \prime}+\frac{\Delta \xi^{2}}{12} x_{\xi}^{2} u^{\prime \prime \prime \prime}+\mathcal{O}\left(\Delta \xi^{4}\right),
$$

which can be written as

$$
u^{\prime \prime}=\widetilde{u^{\prime \prime}}-\underbrace{\frac{\Delta \xi^{2}}{12}\left(4 x_{\xi \xi} u^{\prime \prime \prime}+x_{\xi}^{2} u^{\prime \prime \prime \prime}\right)}_{\tau_{2}}+\mathcal{O}\left(\Delta \xi^{4}\right),
$$


Higher order non-uniform grids for singularly perturbed convection-diffusion-reaction problems

where $\tau_{2}$ is the truncation error for the second derivative approximation. In a similar way, we can write the first derivative term in model (3.1) as:

$$
\frac{g\left(u_{j+1}\right)-g\left(u_{j-1}\right)}{p+q}=g^{\prime}(u)+\frac{p-q}{2 !} g^{\prime \prime}(u)+\frac{p^{2}-p q+q^{2}}{3 !} g^{\prime \prime \prime}(u)+\mathcal{O}\left(\Delta \xi^{4}\right),
$$

from which follows that:

$$
[g(u)]^{\prime}=\underbrace{\frac{g\left(u_{j+1}\right)-g\left(u_{j-1}\right)}{x_{j+1}-x_{j-1}}}_{[g(u)]^{\prime}}+\underbrace{\frac{\Delta \xi^{2}}{6}\left(3 x_{\xi \xi} g^{\prime \prime}(u)+x_{\xi}^{2} g^{\prime \prime \prime}(u)\right)}_{\tau_{1}}+\mathcal{O}\left(\Delta \xi^{4}\right),
$$

where $\tau_{1}$ is the truncation error for the first derivative approximation. By substituting (3.17) and (3.18) into (3.7), we get

$$
\varphi_{j}=\widetilde{\varphi_{j}}+\epsilon \tau_{2}+\tau_{1}=0
$$

where

$$
\widetilde{\varphi_{j}}=\widetilde{u^{\prime \prime}}+\widetilde{[g(u)]^{\prime}}+f(u) .
$$

To obtain a higher order approximation, we set $\epsilon \tau_{2}+\tau_{1}=0$, which is the local truncation error for the SPBVP under consideration (3.1). Finally, after using the expressions for $\tau_{1}$ and $\tau_{2}$, we find the following equation for the first term in the local truncation error of model (3.1):

$$
\frac{\epsilon}{12}\left(4 x_{\xi \xi} u^{\prime \prime \prime}+x_{\xi}^{2} u^{\prime \prime \prime \prime}\right)-\frac{1}{6}\left(3 x_{\xi \xi} g^{\prime \prime}(u)+x_{\xi}^{2} g^{\prime \prime \prime}(u)\right)=0 .
$$

Depending on the functions $g(u)$ and $f(u)$, we are able to find a smooth mapping $x(\xi)$ from this last expression. In a next step we then compute the numerical solution of problem (3.1) on the smooth mapping $x(\xi)$.

\subsubsection{Numerical experiments}

In this section we present the numerical results for linear and nonlinear convection-diffusion SPBVPs (3.1) with boundary layers to validate the proposed numerical strategy, presented in section 3.4. Without loss of generality, we represent $u(x, \epsilon)$ as $u$. To compute the solution numerically, we solve the system (3.7) efficiently using a tridiagonal matrix algorithm on non-uniform grids. For the nonlinear case, we develop an exact optimal non-uniform grid transformation by approximating the nonlinear source term up to linear terms and apply this optimal grid transformation to compute the solution of the nonlinear SPBVP. Furthermore, we compare the results between the uniform (3.3) and non-uniform (3.7) finite difference schemes. In order to measure the quality of the numerical solution, we compute the maximum error (3.20). For nonlinear SPBVPs, we take the maximum value as a reference value which can be obtained from the numerical solution of uniform central discretization system (3.3) at high values of $J=7000$ for decreasing values of $\epsilon$. We measure the accuracy of the numerical solution by computing its distance to the reference solution as

$$
\left\|\varepsilon_{h}\right\|_{\infty} \equiv\left\|u_{h}-u\right\|_{\infty}=\max _{1 \leq j \leq J}\left|u_{j}-u\left(x_{j}\right)\right|
$$

and the order of convergence can be calculated numerically as:

$$
\text { order }=\log _{2}\left[\frac{\left\|\varepsilon_{h}\right\|_{\infty}}{\left\|\varepsilon_{h / 2}\right\|_{\infty}}\right] .
$$

Our proposed numerical strategy shows the main effect of an optimal non-uniform grid transformation $x(\xi)$ for a linear and nonlinear SPBVPs in the following numerical experiments. 


\section{Test problem 1: linear convection-diffusion model}

Consider the singularly perturbed linear convection-diffusion BVP:

$$
\epsilon u^{\prime \prime}-u^{\prime}=0, \quad u(0)=0, \quad u(1)=1
$$

with $g(u)=u$ and $f(u)=0$ in (3.1). This model has the exact solution

$$
u(x)=\frac{\mathrm{e}^{x / \epsilon}-1}{\mathrm{e}^{1 / \epsilon}-1} .
$$

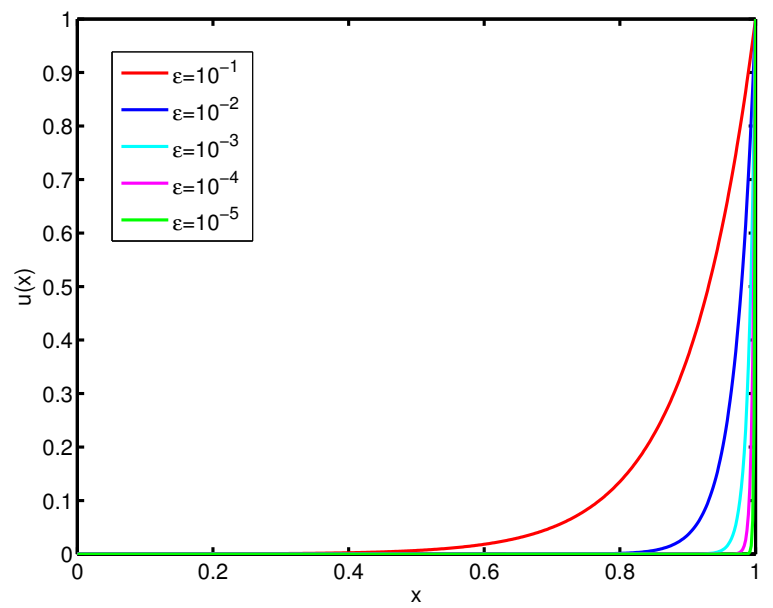

Figure 3.3: Exact solutions of the convection-diffusion problem (3.22) for decreasing values of $\epsilon$.

For $0<\epsilon \ll 1$, the solution is monotonically increasing with a boundary layer near $x=1$. Suppose that we map the steep solution $u(x)$ of the given boundary value problem (3.22) to the milder solution $v(\xi)$ in the new coordinate $\xi$ as is illustrated in Figure 3.2. Model (3.22) is transformed from the physical coordinate $x$ to the computational coordinate $\xi$, via the mapping $x(\xi)$, to:

$$
\frac{\epsilon}{x_{\xi}}\left[\frac{v_{\xi \xi} x_{\xi}-v_{\xi} x_{\xi \xi}}{x_{\xi}^{2}}\right]-\frac{v_{\xi}}{x_{\xi}}=0 .
$$

Assuming that $x_{\xi}>0$ and that the steep solution $u(x)$ is mapped to the linear function $v(\xi)=\xi$ (see Figure 3.5), we obtain from (3.24) the following nonlinear differential equation for $\xi$ :

$$
\epsilon x_{\xi \xi}+x_{\xi}^{2}=0, \quad x(0)=0, x(1)=1 .
$$

which has the exact solution

$$
x(\xi)=\epsilon \ln \left(\xi\left(e^{\frac{1}{\epsilon}}-1\right)+1\right) .
$$

This non-uniform mapping with the grid points $\xi_{j}=j \Delta \xi$ is shown in Figure 3.4 (middle panel). This choice is not optimal, as we will see later. For an optimal choice of $x(\xi)$, we need to do further analysis. To achieve this, we extract the following expression from the local truncation error equation (3.19) with $g(u)=u$ and $f(u)=0$ for the given model (3.22):

$$
\epsilon x_{\xi \xi}+\frac{3}{10} x_{\xi}^{2}=0, \quad x(0)=0, x(1)=1 .
$$


Higher order non-uniform grids for singularly perturbed convection-diffusion-reaction problems

which has the exact solution

$$
x(\xi)=\frac{10}{3} \epsilon \ln \left(\xi\left(e^{\frac{3}{10 \epsilon}}-1\right)+1\right) .
$$

This smooth non-uniform mapping is represented in Figure 3.4 (right panel). The corresponding $v(\xi)$ can be obtained from solving (3.24) by using (3.26), see also in Figure 3.5 (right).
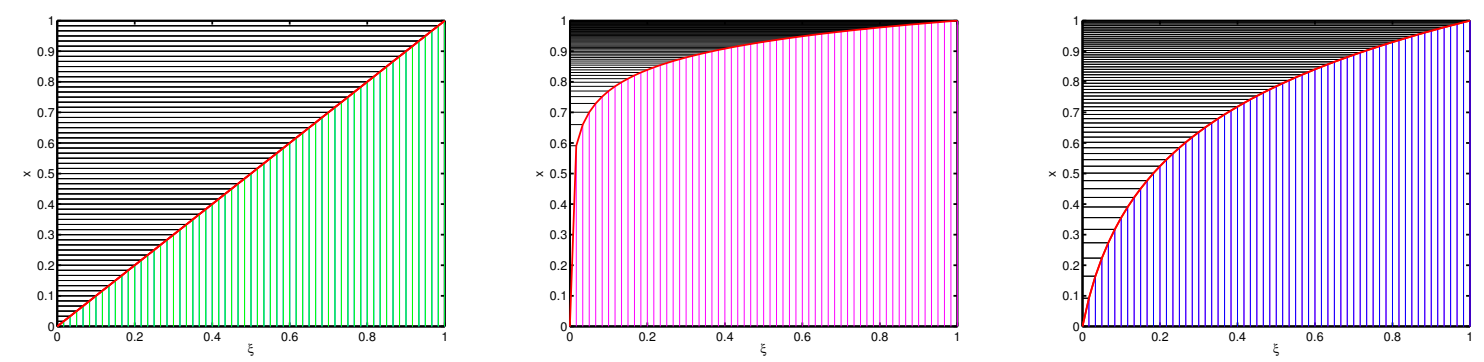

Figure 3.4: The functions $x(\xi)$ for a uniform grid (left), a non-uniform grid (3.25) (middle) and the optimal non-uniform grid using (3.26) (right).

To compute the numerical solution, we solve the discretized system (3.7) for the given boundary value problem (3.22) by using (3.25) and (3.26) respectively. In Tables 3.2 and 3.3, we com-
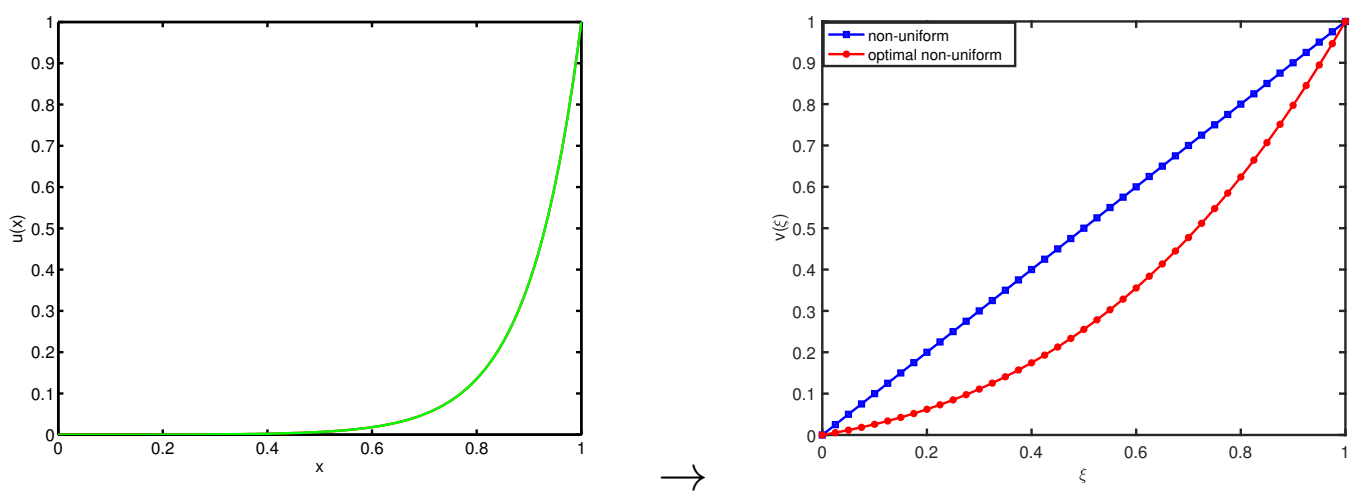

Figure 3.5: In the right panel we see the corresponding functions $v(\xi)$ from non-uniform 3.25 ) and optimal non-uniform grid (3.26) for $\epsilon=0.1$ and the left panel depicts the original function $u(x)$ on uniform grid

pare the results by using uniform and non-uniform grids (3.3), (3.25) and (3.26), respectively. From these results, we observe that the numerical solution with the non-uniform grid (3.25) is more accurate than the uniform case (3.3). but they have the same order of convergence. On the other hand, the non-uniform grid (3.26) behaves exceptionally: the results are fourth order accurate. This transformation (3.26) seems to be optimal, in the sense that we improve the convergence order from $2^{\text {nd }}$ to $4^{\text {th }}$ by choosing a 'better' mapping. It is noticed that when $\epsilon$ decreases, the solution becomes steeper (see Figure 3.3). The maximum error also slightly decreases but we need more grid points to see $2^{\text {nd }}$ or $4^{\text {th }}$ order of convergence (see Table 3.3 for $\epsilon=10^{-2}, 10^{-3}, 10^{-4}, 10^{-5}$ with $J=640$ grid points). 
Table 3.2: Maximum error and convergence order for the linear model (3.22) with $\epsilon=10^{-1}$.

\begin{tabular}{|c||c||c||c||c||c||c|}
\hline \multirow{2}{*}{$J$} & \multicolumn{2}{c||}{ uniform } & \multicolumn{2}{c||}{ non-uniform $(3.25)$} & \multicolumn{2}{c|}{ optimal non-uniform grid $(3.26)$} \\
\cline { 2 - 6 } & error & order & error & order & error & order \\
\hline 10 & 0.0435 & - & $3.3025 \mathrm{e}-05$ & - & $5.6611 \mathrm{e}-06$ & - \\
20 & 0.0087 & 2.3219 & $7.7605 \mathrm{e}-06$ & 2.0893 & $3.6031 \mathrm{e}-07$ & 3.9738 \\
40 & 0.0020 & 2.1210 & $1.8437 \mathrm{e}-06$ & 2.1540 & $2.2321 \mathrm{e}-08$ & 4.0128 \\
80 & $4.9166 \mathrm{e}-04$ & 2.0243 & $4.1671 \mathrm{e}-07$ & 2.0650 & $1.3345 \mathrm{e}-10$ & 4.0640 \\
160 & $1.2122 \mathrm{e}-04$ & 2.0200 & $9.6488 \mathrm{e}-08$ & 2.1106 & $8.1537 \mathrm{e}-11$ & 4.0327 \\
320 & $3.0107 \mathrm{e}-05$ & 2.0095 & $2.3226 \mathrm{e}-08$ & 2.0546 & $4.9879 \mathrm{e}-12$ & 4.0310 \\
640 & $7.5026 \mathrm{e}-06$ & 2.0046 & $5.4692 \mathrm{e}-09$ & 2.0863 & $3.1021 \mathrm{e}-13$ & 4.0071 \\
\hline
\end{tabular}

Table 3.3: Maximum error and convergence order for the linear model (3.22) on decreasing values of $\epsilon$ with $J=640$ grid points.

\begin{tabular}{|c||c||c||c||c||c||c|}
\hline \multirow{2}{*}{$\epsilon$} & \multicolumn{2}{|c||}{ uniform } & \multicolumn{2}{c||}{ non-uniform $(3.25)$} & \multicolumn{2}{c|}{ optimal non-uniform grid $(3.26)$} \\
\cline { 2 - 6 } & error & order & error & order & error & order \\
\hline $10^{-2}$ & $7.3138 \mathrm{e}-04$ & 2.0363 & $3.3664 \mathrm{e}-08$ & 2.0317 & $4.7661 \mathrm{e}-13$ & 4.0407 \\
$10^{-3}$ & 0.0871 & 1.6025 & $1.1521 \mathrm{e}-07$ & 2.0414 & $9.7757 \mathrm{e}-12$ & 4.0067 \\
$10^{-4}$ & 0.2734 & 0.1865 & $6.2397 \mathrm{e}-06$ & 2.0082 & $1.6979 \mathrm{e}-10$ & 3.9559 \\
$10^{-5}$ & 0.5548 & 0.0764 & $6.6461 \mathrm{e}-05$ & 1.9321 & $2.2011 \mathrm{e}-09$ & 3.8456 \\
$10^{-6}$ & 0.6239 & 0.0411 & $8.3132 \mathrm{e}-03$ & 1.7644 & $8.8924 \mathrm{e}-07$ & 3.5668 \\
\hline
\end{tabular}

\section{Test problem 2: nonlinear convection-diffusion-reaction model}

Consider the following singularly perturbed nonlinear convection-diffusion-reaction SPBVP [7] with homogeneous Dirichlet boundary conditions:

$$
\epsilon u^{\prime \prime}+2 u^{\prime}+\mathrm{e}^{u}=0, \quad u(0)=0, \quad u(1)=0 .
$$

Here, we have $g(u)=2 u$ and $f(u)=e^{u}$ in SPBVP (3.1). The solution of this non-linear problem exhibits a boundary-layer behavior at the left boundary at $x=0$ (see also Figure 3.6). It is difficult to compute the optimal choice of the non-uniform mapping $x(\xi)$ for this nonlinear convection diffusion reaction model. To obtain a nearly optimal $x(\xi)$, we approximate the nonlinear term $\mathrm{e}^{u}$ in $(3.27)$ by a Taylor series upto the linear term $1+u$. In the following, we will construct the exact optimal grids $x(\xi)$ for the linearized model with a linear source term and compute with this grid the solution of BVP (3.27). We solve the non-linear discretized system (3.3) on a uniform grid and the scheme (3.7) iteratively and make use of the Matlab direct solver fsolve. To start the iterations, we used a sinusoidal function that satisfies the boundary conditions: $u_{0}=a \sin (\pi x)$ as an initial guess with an amplitude $a$, to be specified for each value of $\epsilon$. By approximating the nonlinear term, the BVP (3.27) can be written as:

$$
\epsilon u^{\prime \prime}+2 u^{\prime}+(1+u)=0, \quad u(0)=0, \quad u(1)=0 .
$$

The following function solves the linearized BVP exactly:

$$
u(x)=C_{1} \mathrm{e}^{\frac{-2+\mu}{2 \epsilon} x}+C_{2} \mathrm{e}^{\frac{-2-\mu}{2 \epsilon} x}-1,
$$

where

$$
\mu=\sqrt{4-4 \epsilon}, \quad C_{1}=\frac{1-\mathrm{e}^{\frac{-2-\mu}{2 \epsilon}}}{\mathrm{e}^{\frac{-2+\mu}{2 \epsilon}}-\mathrm{e}^{\frac{-2-\mu}{2 \epsilon}}}, \quad C_{2}=\frac{\mathrm{e}^{\frac{-2+\mu}{2 \epsilon}}-1}{\mathrm{e}^{\frac{-2+\mu}{2 \epsilon}}-\mathrm{e}^{\frac{-2-\mu}{2 \epsilon}}} .
$$




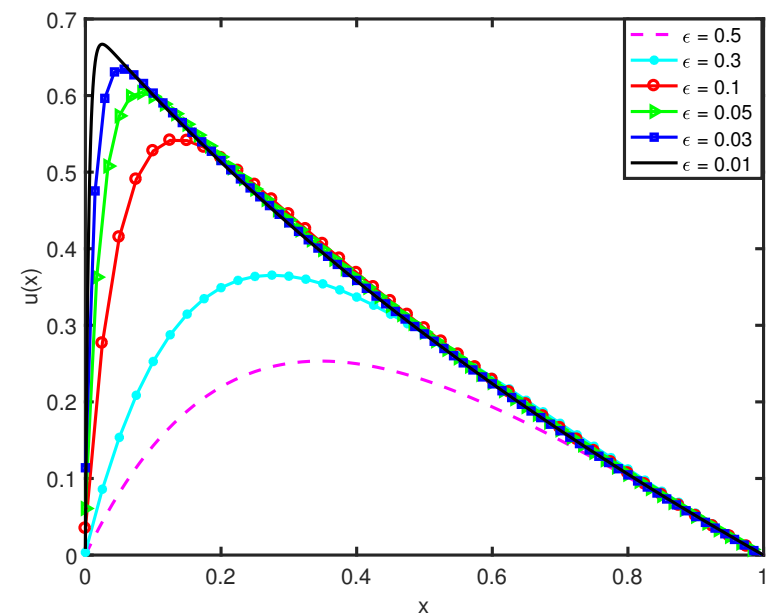

Figure 3.6: Accurate numerical solutions of the nonlinear boundary value problem (3.27) for decreasing values of $\epsilon$.

Note that, since $0<\epsilon \ll 1$, we have $4-4 \epsilon>0$. To find an exact optimal non-uniform grid $x(\xi)$, we describe the truncation error as mentioned in (3.19) with the use of the exact solution (3.28):

$$
\begin{gathered}
\epsilon x_{\xi \xi}\left\{\left(C_{1}(-2+\mu)^{3}+C_{2}(-2-\mu)^{3} e^{\left(-\frac{\mu}{\epsilon}\right) x}\right)-6\left(C_{1}(-2+\mu)^{2}+C_{2}(-2-\mu)^{2} e^{\left(-\frac{\mu}{\epsilon}\right) x}\right)\right\}+ \\
x_{\xi}^{2}\left\{\frac{1}{8}\left(C_{1}(-2+\mu)^{4}+C_{2}(-2-\mu)^{4} e^{\left(-\frac{\mu}{\epsilon}\right) x}\right)-\left(C_{1}(-2+\mu)^{3}+C_{2}(-2-\mu)^{3} e^{\left(-\frac{\mu}{\epsilon}\right) x}\right)\right\}=0 .
\end{gathered}
$$

Assuming that $e^{-\frac{\mu}{\epsilon} x} \approx 1$, we get the expression:

$$
\epsilon x_{\xi \xi}-\frac{B}{8 A} x_{\xi}^{2}=0, \quad x(0)=0, x(1)=1,
$$

where

$$
\begin{gathered}
A=\left(C_{1}(-2+\mu)^{3}+C_{2}(-2-\mu)^{3}\right)-6\left(C_{1}(-2+\mu)^{2}+C_{2}(-2-\mu)^{2}\right), \\
B=\left(C_{1}(-2+\mu)^{4}+C_{2}(-2-\mu)^{4}\right)-8\left(C_{1}(-2+\mu)^{3}+C_{2}(-2-\mu)^{3}\right) .
\end{gathered}
$$

It can be checked that the following expression solves the problem (3.29) exactly (see Figure 3.7):

$$
x(\xi)=\frac{8 A}{B} \epsilon \ln \left(\xi\left(e^{\frac{B}{8 A \epsilon}}-1\right)+1\right) .
$$

The corresponding $v(\xi)$ can be obtained from (3.8) using (3.30) and (3.28). The steep solution $u(x)$ in the $x$-coordinate is transformed to a milder solution $v(\xi)$ in the $\xi$-coordinate (see Figure 3.8).

We compute the numerical solution of model (3.27), by solving the discretized system (3.11) on the optimal non-uniform mapping (3.30). For $0<\epsilon \ll 1$, we compare the results (in the Tables 3.4 and 3.5) for the uniform $x(\xi)=\xi$ and the near-optimal non-uniform grid (3.30), respectively. We observe that the near-optimal non-uniform grid behaves more accurately and is of higher order than for the uniform case.

The numerical results in Table 3.5 illustrate that for decreasing values of $\epsilon$, the second order of accuracy for the uniform is obtained. On the other hand, for the near-optimal case of the non-uniform grid (3.30), we get approximately fourth order of accuracy. For smaller values of $\epsilon$, we need more grid points to get a second or fourth order of convergence. 


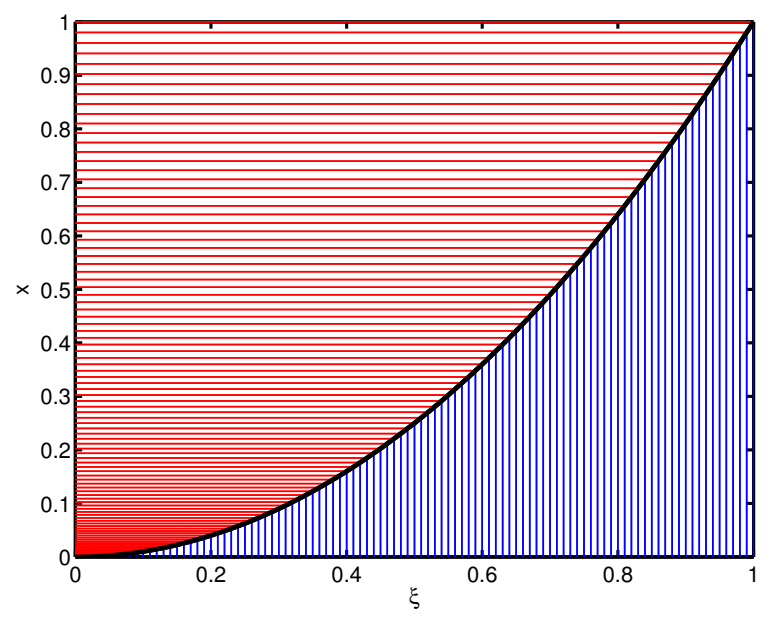

Figure 3.7: Optimal non-uniform grid for model (3.27) with $\epsilon=10^{-1}$.
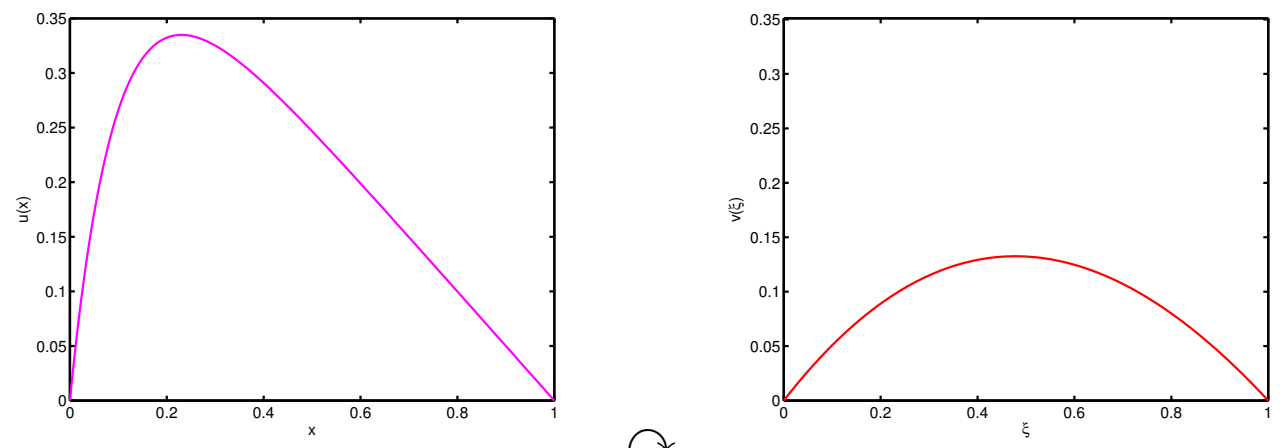

Figure 3.8: Transformation from the steep solution $u(x)$ to the milder solution $v(\xi)$ for model (3.27) with $\epsilon=10^{-1}$.

Table 3.4: Maximum error and convergence order for the model (3.27) with $\epsilon=10^{-1}$.

\begin{tabular}{|c||c||c||c||c|}
\hline \multirow{2}{*}{\multicolumn{1}{|c||}{$J$}} & \multicolumn{2}{c||}{ uniform: $x(\xi)=\xi$} & \multicolumn{2}{c|}{ optimal non-uniform: $(3.30)$} \\
\cline { 2 - 5 } & error & order & error & order \\
\hline \hline 10 & 0.0615 & - & $4.2318 \mathrm{e}-04$ & - \\
20 & 0.0097 & 2.6645 & $3.4352 \mathrm{e}-05$ & 3.6228 \\
40 & 0.0024 & 2.0150 & $2.7312 \mathrm{e}-06$ & 3.6528 \\
80 & $5.6961 \mathrm{e}-04$ & 2.0750 & $2.0112 \mathrm{e}-07$ & 3.7634 \\
160 & $1.3861 \mathrm{e}-04$ & 2.0389 & $1.4231 \mathrm{e}-08$ & 3.8209 \\
320 & $3.3745 \mathrm{e}-05$ & 2.0383 & $9.8878 \mathrm{e}-10$ & 3.8472 \\
640 & $8.3567 \mathrm{e}-06$ & 2.0137 & $6.6543 \mathrm{e}-11$ & 3.9078 \\
\end{tabular}


Higher order non-uniform grids for singularly perturbed convection-diffusion-reaction problems

Table 3.5: Maximum error and convergence order for model (3.27) with grid points $J=640$.

\begin{tabular}{|c||c||c||c||c|}
\hline \multirow{2}{*}{$\epsilon$} & \multicolumn{2}{c||}{ uniform } & \multicolumn{2}{c|}{ optimal non-uniform grid (3.30) } \\
\cline { 2 - 5 } & error & order & error & order \\
\hline $10^{-2}$ & $9.4583 \mathrm{e}-04$ & 1.9679 & $5.6252 \mathrm{e}-09$ & 3.6501 \\
$10^{-3}$ & 0.0525 & 1.7324 & $7.3423 \mathrm{e}-07$ & 3.5615 \\
$10^{-4}$ & 0.1164 & 0.4252 & $2.3286 \mathrm{e}-6$ & 3.4923 \\
$10^{-5}$ & 0.3248 & 0.0826 & $4.7442 \mathrm{e}-05$ & 3.3022 \\
$10^{-6}$ & 0.7416 & 0.0114 & $1.4261 \mathrm{e}-04$ & 3.1044 \\
\hline
\end{tabular}

Test problem 3: nonlinear convection-diffusion-reaction problem with two boundary layers

Consider the nonlinear singularly perturbed convection-diffusion-reaction problem as:

$$
\epsilon u^{\prime \prime}+[2(2 x-1) u]^{\prime}+4 \sin u=0, \quad u(0)=1, \quad u(1)=1
$$

with $g(u)=2(2 x-1) u$ and $f(u)=4 \sin u$. The solution of this nonlinear problem has two boundary-layers, one at $x=0$ and the other at $x=1$. It is clear that when $\epsilon$ decreases, the solution becomes steeper (see also Figure 3.9).

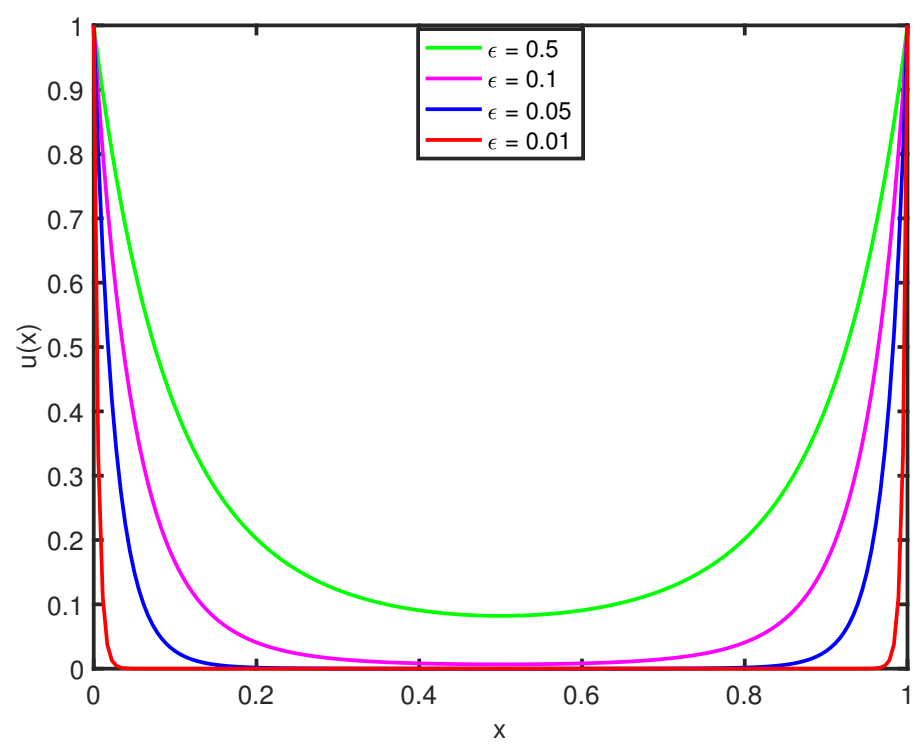

Figure 3.9: Solutions of model (3.31) for decreasing value of $\epsilon$.

In the following, we will construct the exact optimal non-uniform grid from the linearized form of SPBVP (3.31). For the computation of the numerical solution of the nonlinear discretized system of (3.31) iteratively, we adopt the Matlab routine fsolve. The iterations are started with a sinusoidal function that satisfies the boundary conditions: $u_{0}=a \cos (2 \pi x)$. This initial guess has an amplitude $a$, to be specified for each value of $\epsilon$.

By taking $g^{\prime}(u)=2(2 x-1) u^{\prime}+4 u$ and $f(u)=0$, the linearized form of BVP (3.31) reads:

$$
\epsilon u^{\prime \prime}+2(2 x-1) u^{\prime}+4 u=0, \quad u(0)=1, \quad u(1)=1 .
$$


The exact solution of this linearized BVP, given in [74], is:

$$
u(x)=\mathrm{e}^{\frac{-2 x(1-x)}{\epsilon}}
$$

For an exact optimal non-uniform grid $x(\xi)$, we investigate the truncation error as mentioned in (3.19) with the use of the expression (3.32), we get the following:

$$
\epsilon x_{\xi \xi}-\frac{A_{1}}{A_{2}} x_{\xi}^{2}=0, \quad x(0)=0, x(1)=1,
$$

where

$$
A_{1}=\left(16 \xi^{4}-32 \xi^{3}+24 \xi^{2}-24 \epsilon \xi+3 \epsilon^{2}+24 \epsilon \xi^{2}+6 \epsilon-8 \xi+1\right), \quad A_{2}=\epsilon\left(8 \xi^{3}+6 \epsilon \xi-12 \xi^{2}-3 \epsilon+6 \xi-1\right) .
$$

The following expression, calculated with Maple, solves the above linear SPBVP exactly:

$$
x(\xi)=\frac{A_{2}}{A_{1}} \epsilon \ln \left(\frac{A_{1}}{A_{2}}\left(C_{1} \xi+C_{2}\right)\right) .
$$

where

$$
C_{1}=\frac{A_{2}^{2} \mathrm{e}^{\frac{A_{1}}{A_{2}}}\left(\frac{A_{1}}{A_{2}}-1\right)}{A_{1}^{2}}, \quad C_{2}=\frac{A_{2} \mathrm{e}^{\frac{A_{1}}{A_{2}}}}{A_{1}} .
$$

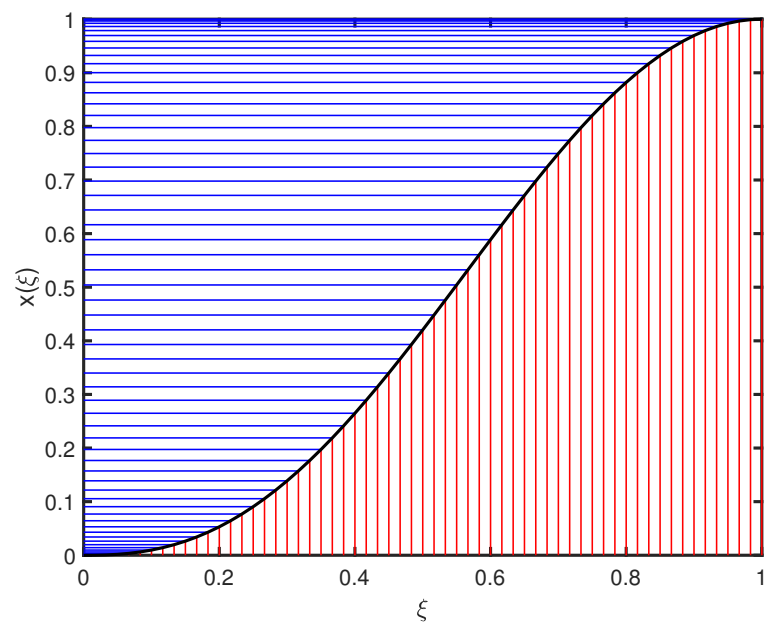

Figure 3.10: Optimal non-uniform grid for model (3.31) with $\epsilon=0.1$

Figure 3.10 shows the optimal mapping (3.34) for the linear SPBVP of (3.31). The milder solution $v(\xi)$ can be obtained from (3.8) using (3.34) and (3.32). The transformation from $x$ coordinate to new $\xi$-coordinate is presented in Figure 3.11.

We compute the numerical solution for SPBVP (3.31) by solving the discretized system (3.11) on the optimal non-uniform grid (3.34) for $0<\epsilon \ll 1$. The numerical results illustrate that the (near-) to optimal non-uniform grid (3.34) transformation performs exceptionally. This transformation is near-optimal, in the sense that we are able to improve the accuracy and convergence order by choosing the appropriate mapping (3.34). For SPBVP (3.31), we get approximately $4^{\text {th }}$ order accuracy and Table 3.6 confirms this behavior.

We observe that for decreasing values of $\epsilon$, the solution becomes more steeper, therefore, we need more grid points, to improve the accuracy and see the convergence order. From Table 3.7 it is noticed that to achieve the same accuracy, the uniform scheme (3.3) needs approximately a factor of 5 to 10 more grid points than the near-optimal non-uniform grid case. The numerical results clearly demonstrate the effectiveness of the proposed near-optimal non-uniform grid. 
Higher order non-uniform grids for singularly perturbed convection-diffusion-reaction problems
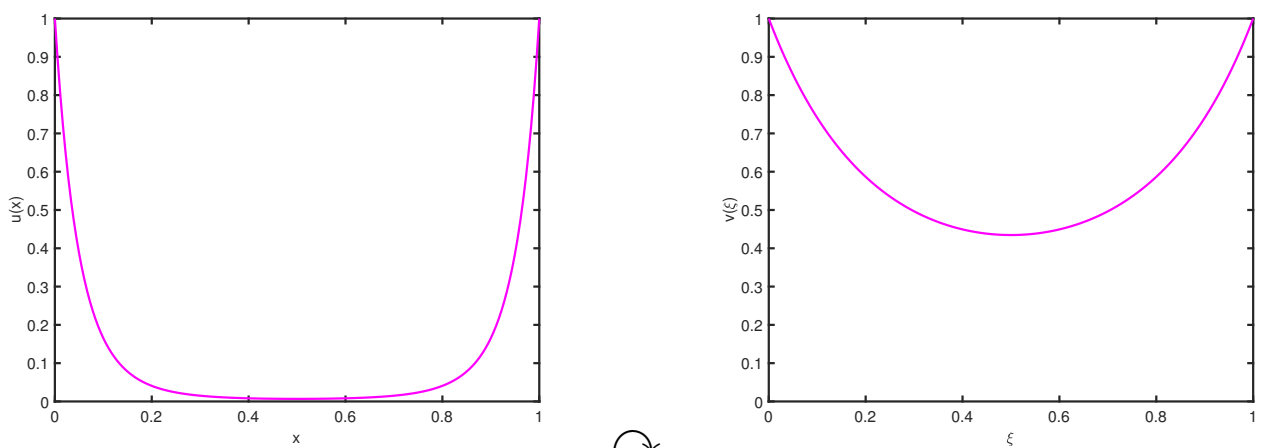

Figure 3.11: Transformation from the steep solution $u(x)$ to the milder solution $v(\xi)$ for model (3.27) with $\epsilon=10^{-1}$.

Table 3.6: Maximum error and convergence order for model (3.31) with $\epsilon=10^{-1}$.

\begin{tabular}{|c||c||c||c||c|}
\hline \multirow{2}{*}{$J$} & \multicolumn{2}{|c||}{ uniform: $x(\xi)=\xi$} & \multicolumn{2}{c|}{ optimal non-uniform: $(3.34)$} \\
\cline { 2 - 5 } & error & order & error & order \\
\hline \hline 10 & 0.4264 & - & 0.0024 & - \\
20 & 0.0745 & 2.5169 & $2.1124 \mathrm{e}-04$ & 3.5061 \\
40 & 0.0154 & 2.2743 & $1.6884 \mathrm{e}-05$ & 3.6452 \\
80 & 0.0036 & 2.0969 & $1.2751 \mathrm{e}-06$ & 3.7270 \\
160 & $8.6664 \mathrm{e}-04$ & 2.0545 & $8.9466 \mathrm{e}-08$ & 3.8331 \\
320 & $2.1342 \mathrm{e}-04$ & 2.0217 & $6.2456 \mathrm{e}-09$ & 3.8404 \\
640 & $5.3289 \mathrm{e}-05$ & 2.0008 & $4.4368 \mathrm{e}-10$ & 3.8152 \\
\hline
\end{tabular}

Table 3.7: Maximum error and convergence order for nonlinear model (3.31) with 640 grid points.

\begin{tabular}{|c||c||c||c||c|}
\hline \multirow{2}{*}{$\epsilon$} & \multicolumn{2}{c||}{ uniform } & \multicolumn{2}{c|}{ optimal non-uniform grid (3.34) } \\
\cline { 2 - 5 } & error & order & error & order \\
\hline $10^{-2}$ & 0.0064 & 1.8432 & $2.7723 \mathrm{e}-09$ & 3.6245 \\
$10^{-3}$ & 0.0716 & 1.3866 & $2.6544 \mathrm{e}-07$ & 3.5421 \\
$10^{-4}$ & 0.1022 & 0.1003 & $1.4338 \mathrm{e}-6$ & 3.3106 \\
$10^{-5}$ & 0.4303 & 0.0474 & $6.6554 \mathrm{e}-04$ & 3.2012 \\
$10^{-6}$ & 0.7226 & 0.0086 & 0.0083 & 3.0035 \\
\hline
\end{tabular}




\subsection{Sixth-order adaptive non-uniform grids}

In this section, we first consider the following singularly-perturbed linear boundary-value problem with inhomogeneous Dirichlet boundary conditions:

$$
\epsilon u^{\prime \prime}-u=0, \quad u(0)=\mathrm{e}^{-\frac{1}{\sqrt{\epsilon}}}, \quad u(1)=1,
$$

which has the exact solution

$$
u(x)=\mathrm{e}^{\frac{x-1}{\sqrt{\epsilon}}} .
$$

For small values of the perturbation parameter $0<\epsilon \ll 1$, the steep solution (3.36) shows a

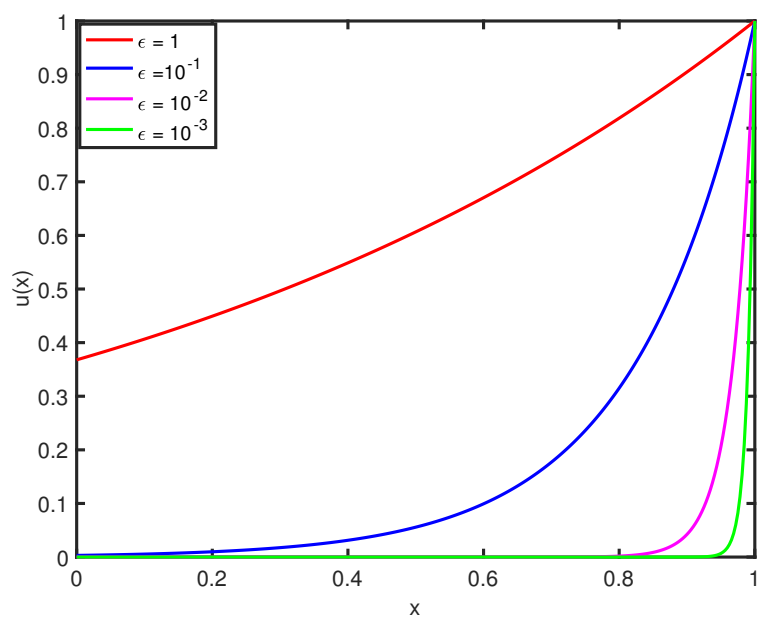

Figure 3.12: Exact solutions of model (3.35) for decreasing values of $\epsilon$.

boundary-layer behavior at $x=1$. This is illustrated in Figure 3.12. However, we will proceed further as if the exact solution is unknown. We will use (3.36) only to assess the quality of a solution.

Approximating the derivatives in model (3.35) by using the expressions from section 3.4.1, yields the following numerical approximation:

$$
\epsilon \frac{\frac{u_{j+1}-u_{j}}{p}-\frac{u_{j}-u_{j-1}}{q}}{h_{j}}-u_{j}=0
$$

with boundary conditions $u_{0}=\mathrm{e}^{-\frac{1}{\sqrt{\epsilon}}}, u_{J}=1$.

The SPBVP (3.35) on the computational domain $I^{*}$ can be discretized on the uniform grid $h$ as (see also section 3.4.2):

$$
\frac{\epsilon}{\mathbb{J}_{j}}\left(\frac{v_{j+1}-v_{j}}{\mathbb{J}_{j+\frac{1}{2}}}-\frac{v_{j}-v_{j-1}}{\mathbb{J}_{j-\frac{1}{2}}}\right)-v_{j}=0, \quad v_{0}=\mathrm{e}^{-\frac{1}{\sqrt{\epsilon}}}, v_{J}=1,
$$

Scheme (3.38) is equivalently with (3.37). We generate an optimal adaptive non-uniform grid based on the equidistribution principle. Further, the numerical solution is computed of the discretized system of given problem (3.35) on the optimal adaptive non-uniform grid to establish a higher order of convergence. 
Higher order non-uniform grids for singularly perturbed convection-diffusion-reaction problems

\subsubsection{Adaptive non-uniform grid generation}

The aim of the equidistribution principle is to concentrate the non-uniformly distributed grid points in the steep regions of the solution (see [22, 53, 149, 147] and references therein). In this principle, the desired mapping $x(\xi)$ is obtained as a solution of the nonlinear problem:

$$
\frac{d}{d \xi}\left[\omega(x) \frac{d x}{d \xi}\right]=0, \quad x(0)=0, \quad x(1)=1,
$$

where $\omega(x)$ is the so-called monitor function. The name equidistribution has to do with the fact that we would like to 'equally distribute' the positive valued and sufficiently smooth function $\omega(x)$ on each non-uniform interval. For this, we first define the grid points

$$
0=x_{0}<x_{1}<x_{2}<\ldots<x_{J-1}<x_{J}=1 .
$$

Next, we determine the grid point distribution such that the contribution of $\omega$ on each subinterval $\left[x_{j-1}, x_{j}\right]$ is equal. A discrete version of (3.39), after integrating once, reads:

$$
\int_{x_{j}}^{x_{j+1}} \omega(x) \mathrm{d} x=\frac{1}{J} \int_{0}^{1} \omega(x) d x, \quad j=0,1, \ldots, J-1 .
$$

In practice, one has to choose the monitor function $\omega(x)$ and solve the nonlinear equation (3.39) to obtain the required mapping $x(\xi)$. Equation (3.39) can be discretized using central finite differences as:

$$
\frac{1}{\Delta \xi}\left[\omega_{j+1 / 2} \frac{p}{\Delta \xi}-\omega_{j-1 / 2} \frac{q}{\Delta \xi}\right]=0, \quad j=1,2, \ldots, J-1
$$

with boundary conditions $x_{0}=0, x_{J}=1$. Equation (3.40) is called a discrete equidistribution principle. The discrete system can be solved efficiently using a tridiagonal matrix algorithm. The iterations are continued until convergence within a prescribed tolerance has been achieved.

\subsubsection{Numerical results}

The aim of this section is to point out that it is possible to develop a central three-point finitedifference scheme on non-uniform grids which exhibits a higher order accuracy than expected and known until now. We solve the discretized system of SPBVP (3.35) on adaptive non-uniform grids based on the equidistribution principle. We establish these higher-order optimal grids ( $4^{\text {th }}$ order and $6^{\text {th }}$ order) with the help of a local truncation error analysis of the discretized system of (3.35). Numerical experiments show that other choices of grid distribution lead to a substantial degradation of the accuracy. Numerical results illustrate the effectiveness of the proposed numerical strategy for linear and nonlinear SPBVPs. The accuracy of the numerical solution is measured by computing its distance to the reference solution as described in equations (3.20) and (3.21).

\section{Case 1: fourth-order accuracy}

The discretization of model (3.35) by approximating the second derivative on non-uniform grids, is given by

$$
\epsilon \frac{\frac{u_{j+1}-u_{j}}{p}-\frac{u_{j}-u_{j-1}}{q}}{\frac{1}{2}(p+q)}-u_{j}=0 .
$$

Scheme (3.41) can be equivalently rewritten as:

$$
\epsilon\left(2 u_{j}-\frac{2 p}{p+q} u_{j-1}-\frac{2 q}{p+q} u_{j+1}\right)+p q u_{j}=0 .
$$


We rewrite expression (3.42):

$$
\left.\epsilon\left(2 u_{j}-R_{1} u_{j-1}-R_{2} u_{j+1}\right)+S_{0} u_{j-1}+S_{1} u_{j}+S_{2} u_{j+1}\right)=0,
$$

where

$$
\begin{gathered}
R_{1}=\frac{2 p}{p+q} \quad \text { and } \quad R_{2}=\frac{2 q}{p+q}, \\
S_{0}=0, \quad S_{1}=p q, \quad S_{2}=0 .
\end{gathered}
$$

For $4^{\text {th }}$ order of convergence, the discretized system is defined as:

$$
\epsilon\left(2 u_{j}-R_{1} u_{j-1}-R_{2} u_{j+1}\right)+S_{1} u_{j}=0 .
$$

This is equivalent to scheme (3.41).

We study the approximation properties of scheme (3.41) on general non-uniform grids. For this, we use the Taylor expansions (3.13), (3.14) and compose the finite differences which appear in (3.41). Assume further that the mapping $x(\xi)$ is sufficiently smooth. The grid difference functions $p$ and $q$ from (3.15) are used to obtain the asymptotic expression:

$$
\epsilon u^{\prime \prime}-u-\epsilon\left(\frac{p-q}{3} u^{\prime \prime \prime}-\frac{p^{2}-p q+q^{2}}{12} u^{(4)}-\frac{(p-q)\left(p^{2}+q^{2}\right.}{60} u^{(5)}+\mathcal{O}\left(\Delta \xi^{4}\right)\right)=0 .
$$

We transform the system $x \mapsto \xi$ so, from $(3.45)$, one gets

$$
-\epsilon \frac{\Delta \xi^{2}}{3}\left[x_{\xi \xi} u^{\prime \prime \prime}+\frac{1}{4} x_{\xi}^{2} u^{\prime \prime \prime \prime}\right]+\mathcal{O}\left(\Delta \xi^{4}\right)=0 .
$$

In general, the scheme (3.41) is second order accurate. However, we notice that the scheme will be fourth order accurate, if the mapping $x(\xi)$ satisfies the following equation:

$$
x_{\xi \xi} u^{\prime \prime \prime}+\frac{1}{4} x_{\xi}^{2} u^{\prime \prime \prime \prime}=0,
$$

where $u(x)$ is a solution of (3.35). Equation (3.47) can be rewritten as:

$$
\left(u^{\prime \prime \prime}\right)^{\frac{3}{4}}\left[\left(u^{\prime \prime \prime}\right)^{\frac{1}{4}} x_{\xi}\right]_{\xi}=0 .
$$

Since $u^{\prime \prime \prime} \propto u^{\prime}$, we obtain $\left[\left(u^{\prime}\right)^{\frac{1}{4}} x_{\xi}\right]_{\xi}=0$. For our numerical simulations, we make the following choice of the monitor function to illustrate the use of the equidistribution principle (see section 3.5.1):

$$
\omega=\left(u^{\prime}\right)^{\frac{1}{4}}
$$

In the equidistribution method, we obtain the mapping $x(\xi)$ from (3.39). For SPBVP (3.35), we can write the monitor function in a generalized form:

$$
\omega_{\eta}(x)=\left(u^{\prime}(x)\right)^{\eta} .
$$

We take the optimal choice of the monitor function (3.48) to establish the $4^{\text {th }}$ order of convergence for model (3.35). The numerical experiments are performed for different choices of $\eta$ in (3.49) and $\epsilon=0.01$.

We slightly change the monitor function by changing the values of $\eta$ and observe that the convergence order also changes. Table 3.8 shows clearly that the optimal result is obtained for $\eta=\frac{1}{4}$. This is the optimal choice to get the higher order of convergence 4 for the scheme (3.41). On the other hand, for other choices of the monitor functions $\omega(x)$ with different $\eta$ in $(3.49)$, we obtain $2^{\text {nd }}$ order of convergence. However, for the choice $\omega=\left(u^{\prime}\right)^{2}$, the convergence of order falls down to $\approx \frac{1}{2}$, which is, of course, to be avoided for practical numerical simulation. As mentioned above, by taking different choices for the monitor functions $\omega$, we observe a difference in accuracy and convergence order of the numerical solutions. An even higher order of convergence can be found by an appropriate choice of the monitor function in the next section. 
Higher order non-uniform grids for singularly perturbed convection-diffusion-reaction problems

Table 3.8: Maximum error and convergence order for Case $I$ for different choices of $\omega$ and $\epsilon=0.01$.

\begin{tabular}{|c||c||c||c||c||c||c||c||c|}
\hline \multirow{2}{*}{$J$} & \multicolumn{2}{|c||}{$\omega=1$} & \multicolumn{2}{c||}{$\omega=\left(u^{\prime}\right)^{\frac{1}{4}}$} & \multicolumn{2}{c||}{$\omega=\left(u^{\prime}\right)^{\frac{1}{5}}$} & \multicolumn{2}{c|}{$\omega=\left(u^{\prime}\right)^{2}$} \\
\cline { 2 - 8 } & error & order & error & order & error & order & error & order \\
\hline 10 & 0.0170 & - & $9.2012 \mathrm{e}-05$ & - & 0.0099 & - & 0.4733 & - \\
20 & 0.0041 & 2.0518 & $5.3023 \mathrm{e}-06$ & 4.1171 & 0.0027 & 1.8745 & 0.3353 & 0.4973 \\
40 & 0.0010 & 2.0356 & $3.1052 \mathrm{e}-07$ & 4.0939 & $6.7831 \mathrm{e}-04$ & 1.9929 & 0.2332 & 0.5239 \\
80 & $2.4523 \mathrm{e}-04$ & 2.0278 & $1.8629 \mathrm{e}-08$ & 4.0591 & $1.6817 \mathrm{e}-04$ & 2.0120 & 0.1575 & 0.5662 \\
160 & $6.0609 \mathrm{e}-05$ & 2.0165 & $1.1383 \mathrm{e}-09$ & 4.0326 & $4.1676 \mathrm{e}-05$ & 2.0126 & 0.0989 & 0.6713 \\
320 & $1.5062 \mathrm{e}-05$ & 2.0086 & $9.6514 \mathrm{e}-11$ & 4.0328 & $1.0365 \mathrm{e}-05$ & 2.0075 & 0.0664 & 0.5748 \\
\hline
\end{tabular}

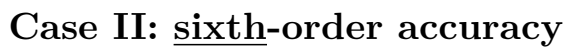

Instead of only using the grid values $\left(x_{j}, u_{j}\right)$ for the approximation of the linear reaction term in (3.35), we consider now the case $S_{0} \neq 0$ and $S_{2} \neq 0$ in (3.43), which means that the reaction term will be approximated on a three-point stencil $\left(x_{j-1}, u_{j-1}\right),\left(x_{j}, u_{j}\right)$ and $\left(x_{j+1}, u_{j+1}\right)$. We expand the various terms of expression (3.43) in a Taylor expansion as mentioned in (3.13) and (3.14). We obtain the following:

$$
\begin{array}{r}
\left(2-R_{1}-R_{2}+S_{1}\right) u_{j}+\Delta \xi\left(R_{1} q-R_{2} p\right) u_{j}^{\prime}+\Delta \xi^{2}\left(-\frac{R_{1}}{2} q^{2}-\frac{R_{2}}{2} p^{2}+S_{0}+S_{2}\right) u_{j}^{\prime \prime}+ \\
\Delta \xi^{3}\left(\frac{R_{1}}{3 !} q^{3}-\frac{R_{2}}{3 !} p^{3}-S_{0} q+S_{2} p\right) u_{j}^{\prime \prime \prime}+\Delta \xi^{4}\left(-\frac{R_{1}}{4 !} q^{4}-\frac{R_{2}}{4 !} p^{4}+S_{0} \frac{q^{2}}{2 !}+S_{2} \frac{p^{2}}{2 !}\right) u_{j}^{\prime \prime \prime \prime}+\ldots=0 .
\end{array}
$$

We now determine the values for $S_{i}(i=1,2,3)$ for Case $I I$, where the coefficients $R_{1}$ and $R_{2}$ are similar to the ones in Case I. We can rewrite expression (3.50) in the following way:

$$
T_{0} u_{j}+\Delta \xi T_{1} u_{j}^{\prime}+\Delta \xi^{2} T_{2} u_{j}^{\prime \prime}+\Delta \xi^{3} T_{3} u_{j}^{\prime \prime \prime}+\Delta \xi^{4} T_{4} u_{j}^{\prime \prime \prime \prime}+\ldots=0,
$$

where the coefficients of $\Delta \xi$ are set as:

$$
\begin{aligned}
& T_{0}=2-R_{1}-R_{2}+S_{1}=0, \\
& T_{1}=R_{1} q-R_{2} p=0, \\
& T_{2}=-\frac{R_{1}}{2} q^{2}-\frac{R_{2}}{2} p^{2}+S_{0}+S_{2}=0, \\
& T_{3}=\frac{R_{1}}{3 !} q^{3}-\frac{R_{2}}{3 !} p^{3}-S_{0} q+S_{2} p=0, \\
& T_{4}=-\frac{R_{1}}{4 !} q^{4}-\frac{R_{2}}{4 !} p^{4}+S_{0} \frac{q^{2}}{2 !}+S_{2} \frac{p^{2}}{2 !}=0 .
\end{aligned}
$$

Making use of the coefficients $T_{1}, \ldots T_{4}$ and $R_{1}, R_{2}$ from $C a s e I$, we obtain the following three expressions for the unknowns coefficients for Case $I I$ :

$$
\begin{aligned}
& S_{0}=\frac{p}{6(p+q)}\left(q^{2}+p q-p^{2}\right), \\
& S_{1}=p q-S_{0}-S_{2}, \\
& S_{2}=\frac{q}{6(p+q)}\left(p^{2}+p q-q^{2}\right) .
\end{aligned}
$$

The terms $R_{1}, R_{2}$ from Case $I$ and equations (3.52) define Case II. For a higher order accuracy, we expand several more terms of the Taylor expansion of equation (3.43) and then estimate the 
terms asymptotically as mentioned in equations (3.13), (3.14), respectively. Finally, equation (3.43) yields:

$$
-\epsilon \frac{\Delta \xi^{4}}{20}\left(x_{\xi \xi} u^{(5)}+\frac{1}{12} x_{\xi}^{2} u^{(6)}\right)+\mathcal{O}\left(\Delta \xi^{6}\right)=0 .
$$

For Case II, we obtain a higher order of accuracy (supra-convergence), if the transformation $x(\xi)$ satisfies the following relation:

$$
x_{\xi \xi} u^{(5)}+\frac{1}{12} x_{\xi}^{2} u^{(6)}=0 .
$$

From this follows the equidistribution principle:

$$
\left[\left(u^{(5)}\right)^{\frac{1}{12}} x_{\xi}\right]_{\xi}=0 .
$$

It is easily checked from (3.36), that $u^{(5)} \propto u^{\prime}$, and we find for Case II the equivalent equation:

$$
\left[\left(u^{\prime}\right)^{\frac{1}{12}} x_{\xi}\right]_{\xi}=0 .
$$

Table 3.9: Maximum error and convergence order for Case II for different choices of $\omega$ and $\epsilon=0.01$.

\begin{tabular}{|c||c||c||c|c||c||c||c||c|}
\hline \multirow{2}{*}{$J$} & \multicolumn{2}{|c||}{$\omega=\left(u^{\prime}\right)^{\frac{1}{2}}$} & \multicolumn{2}{c||}{$\omega=\left(u^{\prime}\right)^{\frac{1}{11}}$} & \multicolumn{2}{c||}{$\omega=\left(u^{\prime}\right)^{\frac{1}{12}}$} & \multicolumn{2}{c|}{$\omega=\left(u^{\prime}\right)^{\frac{1}{13}}$} \\
\cline { 2 - 9 } & error & order & error & order & error & order & error & order \\
\hline 10 & 0.0092 & - & 0.0025 & - & $2.1171 \mathrm{e}-05$ & - & $0.0083-$ & \\
20 & 0.0025 & 1.8797 & $5.8710 \mathrm{e}-04$ & 2.0902 & $3.8724 \mathrm{e}-07$ & 5.7727 & 0.0020 & 2.0531 \\
40 & $6.2523 \mathrm{e}-04$ & 1.9995 & $1.4199 \mathrm{e}-04$ & 2.0478 & $6.7322 \mathrm{e}-09$ & 5.8460 & $4.7668 \mathrm{e}-04$ & 2.0689 \\
80 & $1.5463 \mathrm{e}-04$ & 2.0156 & $3.4757 \mathrm{e}-05$ & 2.0304 & $1.1312 \mathrm{e}-10$ & 5.8952 & $1.1680 \mathrm{e}-04$ & 2.0290 \\
160 & $3.8305 \mathrm{e}-05$ & 2.0132 & $8.5887 \mathrm{e}-06$ & 2.0168 & $1.8454 \mathrm{e}-12$ & 5.9378 & $2.8851 \mathrm{e}-05$ & 2.0173 \\
320 & $9.5462 \mathrm{e}-06$ & 2.0045 & $2.1343 \mathrm{e}-06$ & 2.0087 & $2.8758 \mathrm{e}-14$ & 6.0038 & $7.1685 \mathrm{e}-06$ & 2.0189 \\
\hline
\end{tabular}

Next, we numerically solve the system $(3.43)$ with the monitor function $\omega=\left(u^{\prime}\right)^{\frac{1}{12}}$ from (3.54). As indeed follows from the theory, we get more accurate results and a $6^{\text {th }}$ order accuracy (see Table 3.9). By considering other choices for the monitor function, we observe that by taking $\eta=\frac{1}{2}$ in (3.49), scheme (3.43) suddenly drops to $2^{\text {nd }}$ order of accuracy, the same as or the uniform grid case (see Figure 3.13). Also, for this choice of the monitor function in Case I (see Table 3.8) it gives $4^{\text {th }}$ order accurate solutions. We also demonstrate this by taking slightly changed values of $\eta=\frac{1}{11}$ or $\eta=\frac{1}{13}$ : we obtain second order of accuracy for non-optimal grids. The power $\eta=\frac{1}{12}$ in (3.54) gives the optimal $6^{\text {th }}$ order accuracy, which is the maximum order that can be obtained on a three-point non-uniform stencil. No further improvement of the order can be reached. This follows directly from the analysis of systems (3.51) and (3.52).

\subsubsection{Numerical implementation for a nonlinear problem}

To show the effects on a nonlinear model, we finally consider the SPBVP:

$$
10^{-2} u^{\prime \prime}-\sin (u)=0, \quad u(0)=\mathrm{e}^{-10}, \quad u(1)=1 .
$$

Equidistribution equations $\left(3.40\right.$ with monitor functions $\omega=1, \omega=\left(u^{\prime}\right)^{\frac{1}{4}}$, and $\omega=\left(u^{\prime}\right)^{\frac{1}{12}}$, respectively, are being solved iteratively in combination with (3.43). We cannot find an exact 
Higher order non-uniform grids for singularly perturbed convection-diffusion-reaction problems
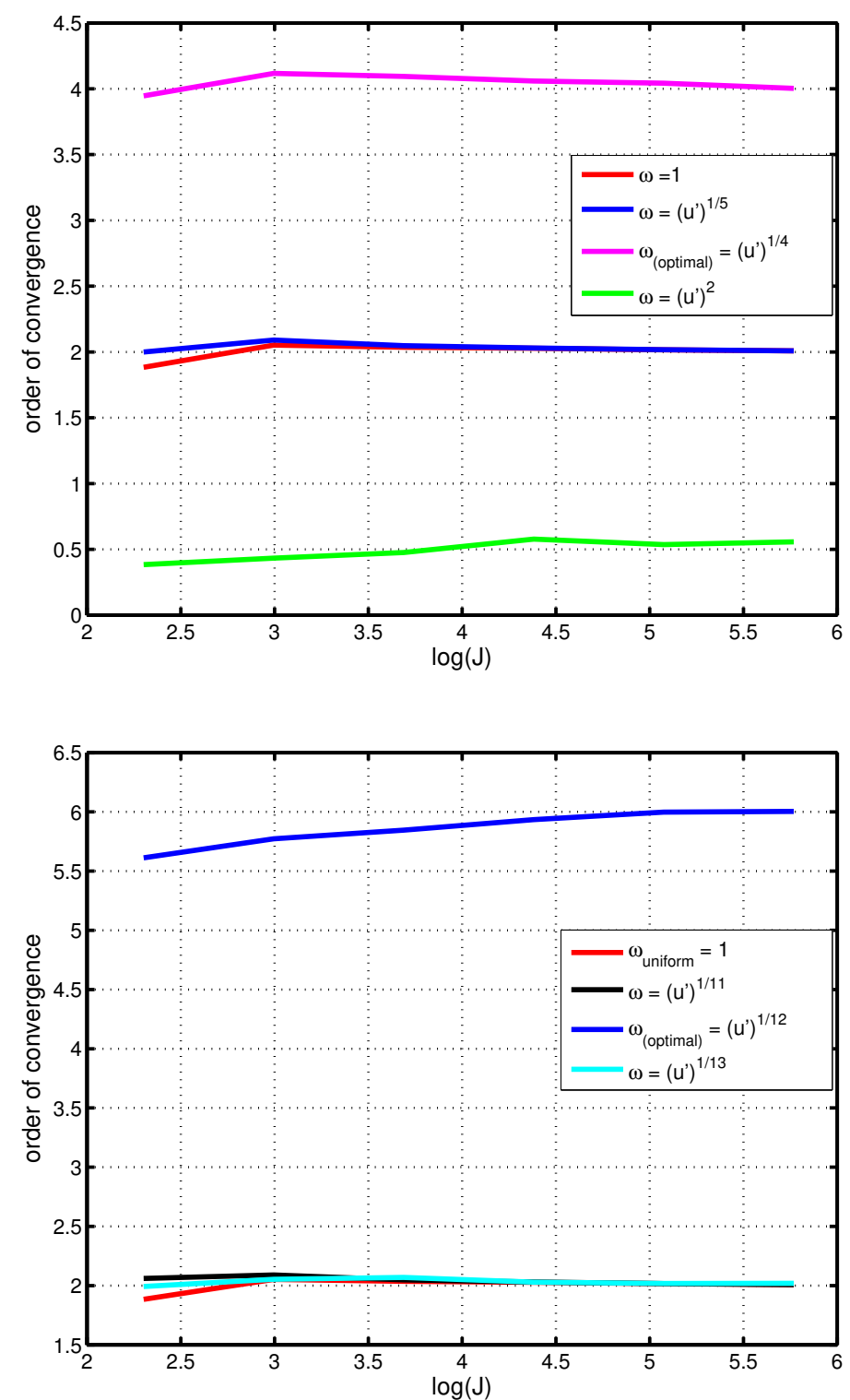

Figure 3.13: Convergence order for several choices of the monitor function $\omega$ for Case I (top) and for Case II (bottom). We observe numerical evidence of the theoretically predicted convergence order, depending on the power in the monitor function. The convergence order can be two for standard choices (top and bottom panel), but also four (top panel) and even six (bottom panel) for special monitor functions, yielding supra-convergence. 
optimal grid transformation as for the linear case. Therefore, we approximate model (3.55) by approximating $\sin (u) \approx u$. Optimal non-uniform grids, for obtaining $4^{\text {th }}$ and $6^{\text {th }}$ order accuracy for the linear case are given by grid transformation (3.49) with $\eta=\frac{1}{4}$ and $\eta=\frac{1}{12}$, respectively. A reference solution of model (3.55) has been obtained by applying a uniform grid with $J=1281$ and the routine $f$ solve from Matlab. The exact solution (3.36) of the linearized model (3.35) has been chosen as the initial guess for the iterative procedure. The numerical results can be found in Table 3.10.

Table 3.10: Maximum error and convergence orders for different choices of the grids for model (3.55): a uniform grid (second order), non-uniform grids with $\eta=\frac{1}{4}$ ( $\approx$ fourth order) and $\eta=\frac{1}{12}$ $\left(\approx 6^{\text {th }}\right.$ order $)$.

\begin{tabular}{|c||c||c||c||c||c||c|}
\hline \multirow{2}{*}{$J$} & \multicolumn{2}{|c||}{ uniform: $\omega=1$} & \multicolumn{2}{c||}{$\omega=\left(u^{\prime}\right)^{\frac{1}{4}}$} & \multicolumn{2}{c|}{$\omega=\left(u^{\prime}\right)^{\frac{1}{12}}$} \\
\cline { 2 - 7 } & error & order & error & order & error & order \\
\hline 20 & 0.0153 & - & $3.4663 \mathrm{e}-04$ & - & $2.6304 \mathrm{e}-05$ & - \\
40 & 0.0038 & 2.0194 & $4.1124 \mathrm{e}-05$ & 3.1663 & $8.4274 \mathrm{e}-07$ & 4.9641 \\
80 & $9.4621 \mathrm{e}-04$ & 2.0057 & $4.3215 \mathrm{e}-06$ & 3.2740 & $2.2635 \mathrm{e}-08$ & 5.2185 \\
160 & $2.3584 \mathrm{e}-04$ & 2.0013 & $3.8656 \mathrm{e}-07$ & 3.4591 & $5.6042 \mathrm{e}-10$ & 5.3359 \\
320 & $5.8774 \mathrm{e}-5$ & 2.0049 & $3.4502 \mathrm{e}-08$ & 3.4967 & $1.3462 \mathrm{e}-11$ & 5.3998 \\
\hline
\end{tabular}

We clearly observe an almost fourth order $(\approx 3.5)$ and almost $6^{\text {th }}$ order $(\approx 5.4)$ accuracy of the proposed non-uniform grid methods. The full orders of four and six cannot be reached, since we approximate the nonlinear function in SPBVP (3.55) by a linear one. Despite the linearization, a significant gain in accuracy can be realized for the nonlinear case as well.

\subsection{Conclusions}

In the present chapter, we proposed a higher order non-uniform finite difference grid, to solve singularly perturbed boundary value problems with steep boundary-layers. We have presented some theoretical properties concerning the extremum values and the asymptotic value at the right boundary point. Traditionally, three-point central finite differences on a uniform grid produce a second order of accuracy. In this chapter, we have developed more accurate and higher order of accuracy non-uniform grid approximations. We have provided several examples of singularly perturbed, both linear and nonlinear, convection-diffusion-reaction problems, which demonstrate the effectiveness of the proposed numerical strategy. We presented a detailed discussion how to obtain a higher order of accuracy by considering a special way of discretizing the given system. The proposed method on optimal non-uniform grids performed exceptionally. We established numerically, not only $4^{\text {th }}$-order but also a $6^{\text {th }}$-order of accuracy by considering only three-point central non-uniform finite differences. We also showed that other choices of the grid distributions lead to a substantial degradation of the accuracy. Numerical experiments have confirmed this behaviour. Comparisons between numerical results illustrate that, to achieve the same accuracy, the proposed method needs approximately a factor of 5-10 fewer grid points than the uniform case. This factor depends, of course, on the value of the small perturbation parameter $\epsilon$ as well. 


\section{Chapter 4}

\section{An efficient nonlinear multigrid scheme for two-dimensional boundary value problems}

\subsection{Introduction}

In this chapter, we consider the nonlinear boundary value problem (BVP):

$$
\begin{aligned}
& \Delta u+\lambda f(u)=0, \quad(x, y) \in \Omega, \\
& \left.u\right|_{\partial \Omega}=0,
\end{aligned}
$$

where $u=u(x, y), \lambda>0$ and $\Omega=[0,1]^{2}$ with boundary $\partial \Omega$. For the special choice $f(u)=\mathrm{e}^{u}$ the BVP (4.1) is a classical two-dimensional Gelfand-Bratu (GB) equation [42, 17, 18]. The GB model appears in a number of applications such as thermo-electro-hydrodynamics models [137], nanotechnology, radioactive heat transfer, chemical reaction theory, fuel ignition models [11], thermal reaction processes, the Chandrasekhar model [24], and in elasticity theory [120].

In the past two decades, several numerical methods have been proposed for the Gelfand-Bratu (Bratu) problem including multilevel continuation techniques [94, multilevel adaptive methods [15], variational iterative methods [9], explicit radial solutions [80], iterative quadrature methods [110], the WR-method [126], nonstandard compact schemes [51], wavelet methods [144], arclength continuation with multigrid [23], a Sinc-Galerkin method [112], pseudospectral methods [13], techniques for the calculation of turning points [98], the variational Iteration method [27]. Most numerical solutions of these methods converge only to the first (lower) solution for $0<$ $\lambda<\lambda_{c}$. Moreover, the mentioned methods lose significantly their accuracy when $\lambda$ approaches the critical value $\lambda_{c}$. In [96], a finite difference method with a nonlinear solver is employed for a 2D Bratu model, in which the author only showed numerical solutions for a grid size $h=1 / 10$.

It is well known that the multigrid (MG) idea is based on two principles: an error smoothing on fine grids and followed by coarse grid corrections. MG schemes are efficient and fast elliptic equations solvers, in general. One approach is to construct a MG scheme on the basis of a global Newton linearization and then solve the resulting system with a linear multigrid scheme [47] or with a Newton- Krylov method. Global linearization techniques that use multigrid as a linear solver or as a preconditioner for Krylov methods are presented in [10, 49, 115]. Alternatively, MG can be applied directly to nonlinear elliptic equations without the use of a global linearization.

This chapter is based on an article in press as [60]: S. Iqbal and P. A. Zegeling, Journal of Applied Mathematics and Computation, 2020. 
This is known as the full approximation storage (FAS) [15]. In FAS-MG, linearization of the nonlinear problem is treated locally in the relaxation step on different grid sizes.

In the literature, only a few multigrid schemes could obtain both solutions of the Bratu problem. The convergence of the first (lower) solution can be successfully obtained by the classical FAS-MG scheme with a Gauss-Seidel smoother. However, the convergence rate of this multigrid solver is very slow and the method could even diverge. This unstable convergence behaviour could be caused by the diagonal element in the exponential form and the linearized system may then become indefinite as the diagonal dominance of the Jacobian matrix is lost [62]. The Jacobian matrix depends on the solution values, the parameter $\lambda$ and the grid size $h$. In that case, Gauss-Seidel is no longer the right choice as a smoother. Hackbusch [48] applied two versions of the nonlinear multigrid scheme, FAS-MG and a nonlinear multigrid method (NMGM). He successfully found the upper solution, but only for large grid sizes: $h=1 / 4$ and $h=1 / 8$. Washio and Oosterlee [138] presented a nonlinear Krylov acceleration method as the outer iteration with a MG preconditioner for the 2D Gelfand-Bratu problem for small values of $\lambda$ with different choices of the smoother. They mentioned that no numerical second solution that exists on the grid of size $h=1 / 4$ for small values of $\lambda$. So the size $h=1 / 8$ was chosen as the coarsest grid fine enough for a second solution to exist.

To handle the unstable convergence problem, in a previous numerical MG study [97], two Krylov methods as smoothers were employed for solving the Bratu problem. For small values of the bifurcation parameter $\lambda$, the Newton-MG method, FMG-FAS and FMG-NMGM with the two Krylov methods as smoothers and $u_{c}$ as an initial guess, were used to obtain both solutions of the Bratu problem in [97]. This MG was still unable to achieve the desired accuracy for small grid sizes $h$.

The present work is an extension of our 1D case, see Chapter 2 and [148]. We are aiming to extend these numerical results to the 2D Gelfand-Bratu problem. Particularly, one of the goals is to obtain new types of solutions, as we did in the 1D case [148]. To achieve this, we propose three numerical methods: a finite difference (FD) based method, Newton-MG and FAS-MG, to compute all possible solutions of the given nonlinear BVP (4.1). These methods successfully handle the mentioned convergence difficulties. In particular, we are able to obtain the upper solution of the two-dimensional Bratu problem for small grid sizes $h$ and for small values of $\lambda$. In the FD-method, we use the Matlab nonlinear solver fsolve to solve the nonlinear system of equations and in MG methods, MINRES is used as a relaxation smoother. Further, we employ the sinusoidal function as an initial approximation for the three proposed numerical methods. Numerical implementation of these methods is provided in more detail, see Section 4.3. The main contribution of the present work is computation of more accurate solutions and confirmation of the convergence of the second branch of solutions (the upper solutions) for small values of $\lambda$. We obtain the desired accuracy on decreasing value of grids size $h$ with the help of proposed numerical strategies. The problem of the indefinite Jacobian matrix is solved successfully for large values of the solution, which correspond with the upper solution in the model. To achieve this, we use the MINRES method as a smoothing relaxation in MG methods and a sinusoidal function as an initial guess. Moreover, we present new types of solutions: periodic and semi-periodic solutions. For special cases of the Gelfand-Bratu problem we also obtain multiple solutions by approximating the nonlinear term. We conjecture that the number of solutions may vary between 0 and $\infty$ depending on the model parameter $\lambda$. The convergence of a numerical method to find new multiplicities of solutions, especially for small values of $\lambda$ on a large number of grid points, is of great importance. We present the numerical convergence of these new multiple solutions by employing the proposed numerical methods.

The organization of this chapter is follows. In section 4.2, we present three numerical methods: a finite difference(FD) based method, Newton-MG and FAS-MG, respectively for computing 
the numerical solutions of the two-dimensional GB model. Numerical experiments in section 4.3, illustrate the bifurcation behaviour of the GB model and demonstrate the convergence of all types of solutions: lower, upper, periodic and semi-periodic for different values of $\lambda$. Furthermore, numerical results show the performance of the proposed methods. Finally, concluding remarks are presented in section 4.4 .

\subsection{A description of the three numerical methods}

To compute numerical solutions of the nonlinear boundary value problem (4.1), we present three numerical methods: a finite difference based method, Newton-MG and FAS-MG. More details of these numerical methods will be discussed in the following sections.

\subsubsection{Method 1: a central finite difference approximation with fsolve.m}

Our first numerical method computes solutions with a central finite difference (FD) scheme. On a uniform spatial grid on the domain $[0,1]^{2}$ we generate the grid points by:

$$
x_{i}=i h, \quad y_{j}=j h, \quad i, j=0,1,2, \ldots J, \quad \text { with } h=\frac{1}{J} .
$$

We denote by $u_{i, j}$ the discrete approximation of the exact (unknown) values $u\left(x_{i}, y_{j}\right)$ Applying a second-order central finite difference approximation on the uniform grid $\Omega_{h}$, yields:

$$
\underbrace{\frac{u_{i+1, j}+u_{i-1, j}+u_{i, j+1}+u_{i, j-1}-4 u_{i, j}}{h^{2}}}_{\mathcal{A} \mathrm{u}}+\lambda f\left(u_{i, j}\right)=0, \quad i, j=1,2, \ldots, J-1,
$$

with the Dirichlet boundary conditions: $u_{0, j}=u_{J, j}=u_{i, 0}=u_{i, J}=0$. The FD scheme at the grid points $\left(x_{i}, y_{j}\right)$ makes use of the five grid points, $\left(x_{i-1}, y_{j}\right),\left(x_{i+1}, y_{j}\right),\left(x_{i}, y_{j-1}\right),\left(x_{i}, y_{j+1}\right)$ and $\left(x_{i}, y_{j}\right)$ and the corresponding coefficient matrix $\mathcal{A}$ becomes block-tridiagonal:

$$
\mathcal{A}=\frac{1}{h^{2}}\left[\begin{array}{cccc}
\mathcal{B} & \mathcal{I} & O & O \\
\mathcal{I} & \mathcal{B} & \mathcal{I} & O \\
\vdots & \vdots & \ddots & \vdots \\
O & O & \mathcal{I} & \mathcal{B}
\end{array}\right]_{(J-1)^{2} \times(J-1)^{2}} \quad \text { with } \quad \mathcal{B}=\left[\begin{array}{cccc}
-4 & 1 & 0 & 0 \\
1 & -4 & 1 & 0 \\
\vdots & \vdots & \ddots & \vdots \\
0 & 0 & 1 & -4
\end{array}\right]_{(J-1) \times(J-1)}
$$

where $\mathcal{I}$ and $O$ are the $(J-1) \times(J-1)$ identity and zero matrices, respectively. In the next step, we solve the discretized system of algebraic equations (4.2) to obtain the approximate solution at each grid point, Finally, the resulting nonlinear system is solved iteratively by applying the nonlinear solver from matlab: fsolve.m. This nonlinear solver starts at initial guess $u^{0}$ and finds the approximate solution of the nonlinear system of equations at certain accuracy. We set the termination tolerance to be: $t o l=10^{-10}$ and the initial approximation is chosen as (see also in the $1 \mathrm{D}$ case $[148])$.

$$
u_{\text {initial }}=u^{0}=\alpha \sin \left(k \pi x_{i}\right) \sin \left(k \pi y_{j}\right),
$$

with an amplitude $\alpha$ and frequency $k$. These numerical parameters need to be specified for each experiment separately. Knowing that the maximum solution $u_{c}$, the lower solution requires $\alpha<u_{c}\left(\frac{1}{2}, \frac{1}{2}\right)$ and the upper solution requires $\alpha>u_{c}\left(\frac{1}{2}, \frac{1}{2}\right)$ for numerical experiments. The other parameter specifies $k=1$ for lower solutions and $k \geq 1$ for upper solutions. This approach is simple and easy to implement numerically for solving the nonlinear system of equations (4.2). 
However the method is not efficient particularly for small values of $\lambda$ and increasing problem size. In such situations, convergence of the solution becomes very slow and sometimes prevents obtaining the set accuracy. To handle this difficulty, we choose the numerical parameters $\alpha$ and $k$ very carefully, as fsolve is very sensitive to the initial guess, especially for small values of $\lambda$ on an increasing number of grid points.

Multigrid (MG) schemes are efficient and fast elliptic equation solvers, in general. To enhance the accuracy and efficiency, we propose to choose the same initial guess for MG methods as used in the current approach (FD method).

\subsubsection{Multigrid methods}

Multigrid methods have been developed for solving nonlinear partial differential equations (PDE) efficiently [131, 140, 82, 124]. For solving the discretized system of the nonlinear boundary value problem (4.1), we discuss two multigrid approaches. The first approach is a Newtonmultigrid method, in which global linearization is naturally applied, like by Newton's method. After having linearized the PDE problem, in each linearization step, the linear multigrid method can be used for solving the Jacobian system. The second approach is known as the full approximation storage (FAS), in which the multigrid method is applied directly to the given nonlinear problem. In the FAS approach, instead of solving an equation for corrections of the solution on a coarse grid, the original equation is prescribed and full solutions are also computed on coarse grids. In the next two subsections we will describe both multigrid methods in more detail.

\section{Method 2: a Newton-multigrid method (Newton-MG)}

Consider the nonlinear system of equations (4.2) on fine grid $h$ to be solved as:

$$
N_{h}\left(\mathrm{u}_{h}\right)=0
$$

where $N($.$) denotes the discrete version of the nonlinear operator, and is defined as$

$$
N(\mathrm{u})=\mathcal{A} \mathrm{u}+\lambda f(\mathrm{u}) .
$$

The residual on fine grid $h$ can be rewritten as:

$$
r_{h}\left(\mathrm{u}_{h}\right)=0-N_{h}\left(\mathrm{u}_{h}\right)
$$

The Newton iteration $k$, for solving equation (4.4), is characterized by

$$
\mathrm{u}_{h}^{k+1}=\mathrm{u}_{h}^{k}+\underbrace{\left(\mathbb{J}\left(\mathrm{u}_{h}^{k}\right)\right)^{-1} r_{h}\left(\mathrm{u}_{h}^{k}\right)}_{e_{h}^{k}}, \quad k=0,1,2,3, \ldots
$$

The current approximation of the nonlinear system of equations (4.4) can be obtained by solving (4.5). For this, the following linear system is to be solved for $e_{h}^{k}$ as:

$$
\mathbb{J}\left(\mathrm{u}_{h}^{k}\right) e_{h}^{k}=r_{h}\left(\mathrm{u}_{h}^{k}\right),
$$

where $\mathbb{J}\left(\mathrm{u}_{h}^{k}\right)=N_{h}^{\prime}\left(\mathrm{u}_{h}^{k}\right)$, is the Jacobian matrix of the nonlinear system and $r_{h}\left(\mathrm{u}_{h}^{k}\right)=-N_{h}\left(\mathrm{u}_{h}^{k}\right)$. Within each Newton iteration, we need to calculate the exact Jacobian matrix $\mathbb{J}\left(u_{h}\right)$ and its inverse. This process is, of course, time consuming, especially for large scale systems. This problem has led to the development of different variants of Newton's method. Solving (4.6) approximately by a chosen iterative method, is called an Inexact Newton Method. The most widely used inexact Newton method, is the Newton multigrid scheme, that makes use of an 
iterative method to solve the Jacobian system. The Jacobian matrix for (4.6) of the nonlinear system of equations (4.4) reads:

$$
\mathbb{J}\left(\mathrm{u}_{h}\right)=\left[\begin{array}{cccc}
\frac{4}{h^{2}} & -\frac{1}{h^{2}} & 0 & 0 \\
-\frac{1}{h^{2}} & \frac{4}{h^{2}} & -\frac{1}{h^{2}} & 0 \\
\vdots & \vdots & \ddots & \vdots \\
0 & 0 & -\frac{1}{h^{2}} & \frac{4}{h^{2}}
\end{array}\right]_{(J-1) \times(J-1)}-\lambda * \operatorname{diag}\left(f\left(u_{i, j}\right)\right) .
$$

To reduce the time of calculating the Jacobian matrix, it is possible to divide the steps into two parts. In (4.7), the first part is independent of the solution $u$. Therefore, it needs to be calculated only once, whereas the second part uses a diagonal matrix that depends on the solution $u$ and needs to be calculated in each iteration. At each step of the Newton-MG method, we employ Newton's method for the outer iteration and a linear multigrid method for the inner iteration for solving (4.6).

Newton-MG, the linear MG approach, initially solves equation (4.6) by an iterative method (a relaxation smoother). We use the MINRES method as a smoothing relaxation in Newton-MG. We apply a Newton-MG-MINRES method with Nested iterations for computing the numerical results of problem (4.1). It is well known that the Newton method converges if the initial approximation is close enough to the desired solution. For this initial approximation (4.3) is used for the Newton-MG method. The numerical parameters $\alpha$ and $k$ need to be specified for each numerical experiment (see Section 4.3). Numerical results indicate the effectiveness of Newton-MG with MINRES and an appropriate initial guess.

\section{Method 3: a nonlinear multigrid method}

Although the Newton-multigrid method is an effective method, it does not make use of multigrid ideas directly to solve the discretized nonlinear system of equations. Much more memory is required for the Newton-MG method for the storage of the fine and coarse level Jacobian values. In the following approach, the multigrid scheme is applied directly to the full nonlinear problem. The nonlinear multigrid algorithm is called full approximation storage (FAS) (see also Brandt [15]). In the numerical scheme the nonlinear problem is solved at each level of the grid via multigrid cycles. In this work, we consider a discretized nonlinear system of equations (4.4) on the original fine grid $h$. Two grids, $h$ and $2 h$ are used to simplify notation for fine and coarse grids. In FAS-MG, suppose $\mathrm{u}_{h}$ is the approximate solution on the fine grid. The solution process may start with an iterative relaxation on the fine grid. Then, the fine grid residual is transferred by a restriction operator $R_{h}^{2 h}$ to a coarse grid. Solve the coarse grid error problem to eliminate the corresponding error. This error is interpolated by linear interpolation operator $I_{2 h}^{h}$ to the fine grid and further use to correct the fine grid approximation. In the present work, we choose to linearize the continuous problem and then apply MINRES method as a smoother for the discretized linearized equation, see also [138, 97]. The linearized equation for the nonlinear boundary-value problem (4.1) reads:

$$
\Delta \mathrm{u}+\lambda f(\tilde{\mathrm{u}}) \mathrm{u}=\lambda(\tilde{\mathrm{u}}-1) f(\tilde{\mathrm{u}}),
$$

where $\tilde{\mathrm{u}}$ is the current approximate solution of $\mathrm{u}$ for the problem (4.1). Assume that step $\nu_{1}$ and step $\nu_{2}$ are the pre-smoothing and post-smoothing steps, respectively. The complete FAS-MG two grid V-Cycle is given as algorithm 1: 


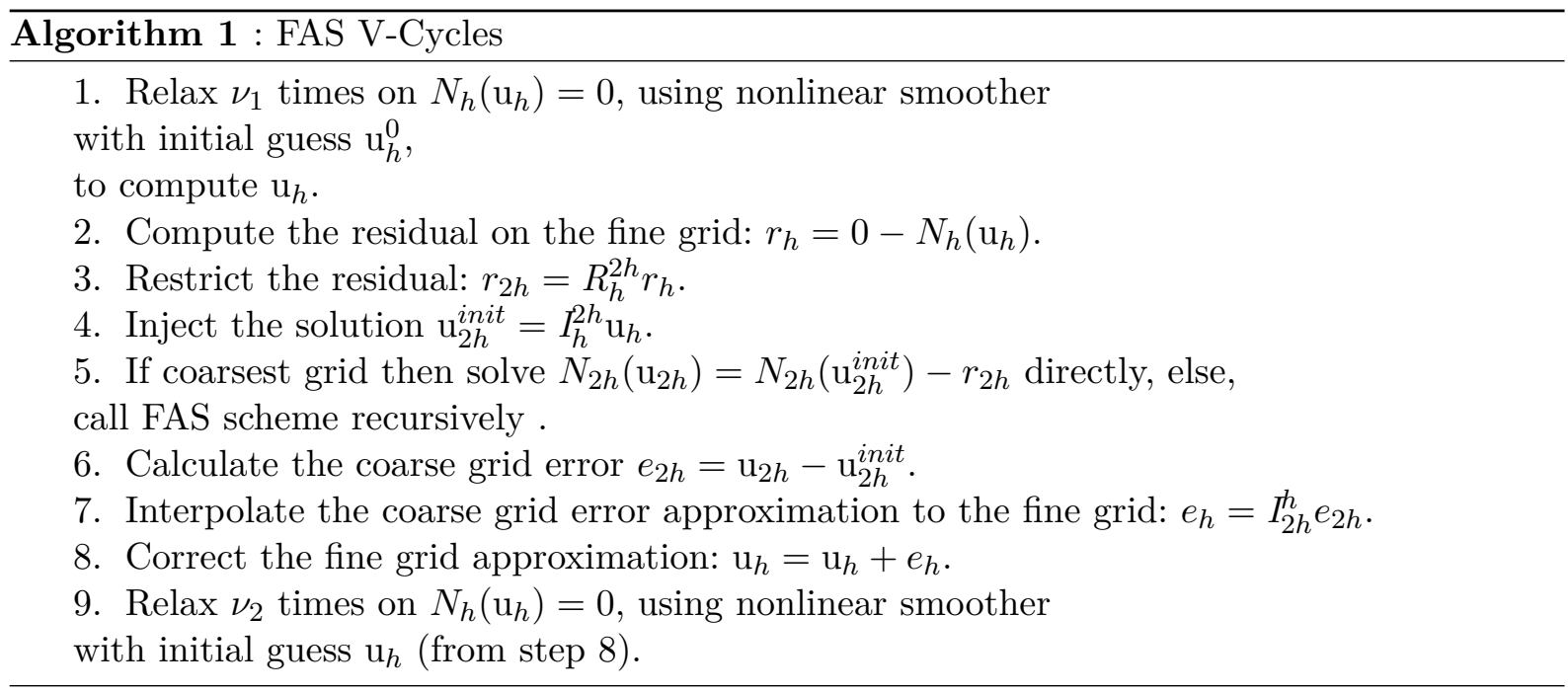

In FAS-MG, each grid problem will itself be a nonlinear differential equation at each grid level. In the present work, for inter-grid transfer, we use bilinear interpolation $I_{h}^{2 h}$ and fullweighting restriction $R_{h}^{2 h}$ operators. For the numerical experiments, we choose the initial guess (4.3) and a MINRES method as the smoothing relaxation as used in the Newton-MG method. Numerical results in Section 4.3 show the improvement in efficiency and the accuracy of the FAS-MG method.

\subsection{Numerical experiments}

In this section, we present numerical experiments for the three numerical methods applied to the BVP (4.1). For the computation of numerical solutions of GB model (4.1), we employ for each experiment: FD scheme with nonlinear solver fsolve, Newton-MG within nested iterations and in FAS-MG, we use the $\mathrm{V}(2,2)$ cycle. In both MG approaches, the MINRES method is used as a relaxation smoother (both for pre and post relaxation). We choose (4.3) as an initial approximation with the numerical parameters $\alpha$ and $k$ for numerical computations of solutions. Knowing that the maximum solution $u_{c} \approx 1.392422635$, the lower solution requires $\alpha<u_{c}$ with the smaller values for small value of $\lambda$. The upper solution requires $\alpha>u_{c}$ with higher values for smaller values of $\lambda$ in the numerical experiments. We choose the value of parameter $k=1$ for lower solution and $k \geq 1$ for upper solutions (multiplicity of solutions). For the numerical results, the residual norm $\|r\|$ is defined, so that the scaling is independent of the grid size $h$ :

$$
\|r\|=\sqrt{\frac{\sum_{i, j} r_{i, j}^{2}}{J}},
$$

and the nonlinear stopping criterium reads: $\|r\| \leq t o l=10^{-10}$. The tolerance of the linear multigrid method within the Newton iterations is set to be $10^{-8}$. The following numerical experiments illustrate the convergence of all types of numerical solutions (lower, upper, periodic and semi-periodic) and demonstrate the effectiveness of the proposed numerical methods for all parameter values $\lambda \in\left(0, \lambda_{c}\right]$. Furthermore, we examine the numerical bifurcation behaviour of the two-dimensional Bratu problem by approximating the nonlinear term and identify the existence of multiple solutions. Numerical results show the comparison of the performance of the three numerical strategies for small values of $\lambda$ on an increasing number of grid points. 


\subsubsection{Experiment 1: the two-dimensional Bratu problem}

Consider the classical two-dimensional Bratu model with homogeneous Dirichlet boundary conditions on the domain $\Omega=[0,1]^{2}$ :

$$
\begin{aligned}
& \Delta u+\lambda \mathrm{e}^{u}=0, \quad(x, y) \in \Omega, \\
& \left.u\right|_{\partial \Omega}=0
\end{aligned}
$$

where $u=u(x, y)$ and $\lambda \geq 0$. Unlike the situation in the one-dimensional problem, see Chapter 2 and [148], no exact solution $u(x, y)$ is known for (4.9). Note that the solution, in the twodimensional case depends heavily on the parameter $\lambda$. The Bratu problem (4.9) has no, one or two solutions, leading to two branches (lower solution and upper solution), for $\lambda>\lambda_{c}, \lambda=\lambda_{c}$ or $\lambda<\lambda_{c}$ respectively as described in Figure 4.1. The value of the turning point $\lambda_{c} \approx 6.808124423$ can be calculated numerically by using a Newton method (also reported in [98, 94]). The solutions of model (4.9) attain their maximum at, and are point-symmetric around, $\left(\frac{1}{2}, \frac{1}{2}\right)$.
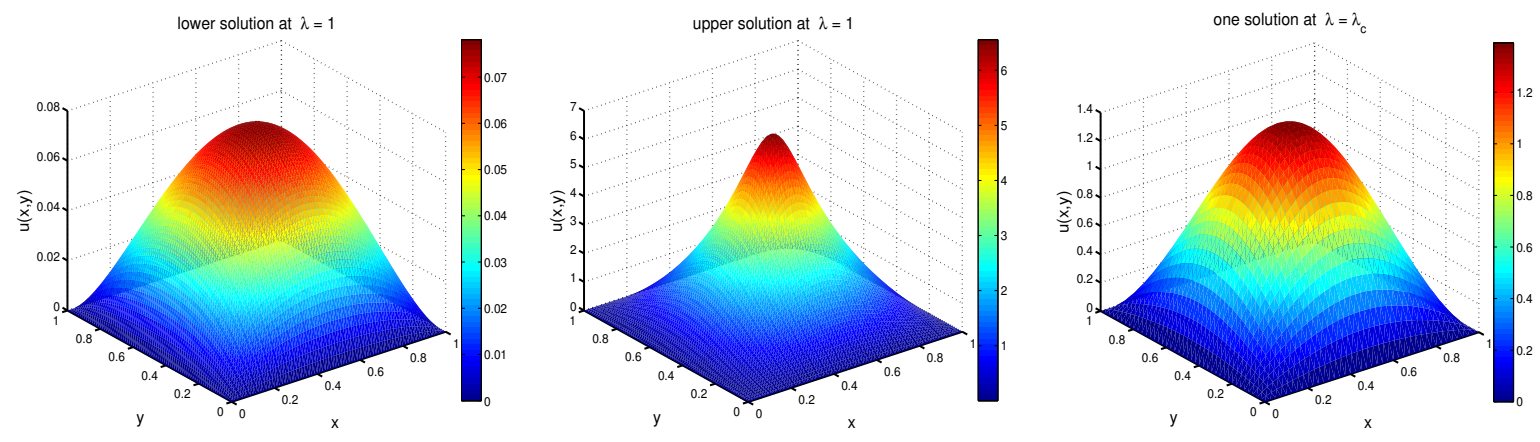

Figure 4.1: Two solutions for $\lambda=1$ (left and middle panel) and the unique solution for $\lambda=\lambda_{c}$ (right panel) of the Bratu-model (4.9) using FAS-MG on $161 \times 161$ grid points.

In earlier numerical approaches [94, 9, 126, 80], researchers reported that the convergence to the lower solution can easily be obtained for all values of $0<\lambda \leq \lambda_{c}$. Table 4.1 shows the performance of three proposed numerical methods to obtain the lower solution of problem (4.9) for $\lambda=1$ on increasing grid points. Results indicate that the three described methods successfully obtain the convergence of lower solution. However, FAS-MG and Newton-MG methods are more efficient than FD-method with respect to time.

Table 4.1: The number of iterations and the CPU time in seconds for the lower solution of model

\begin{tabular}{|c|c|c|c|c|c|c|}
\hline Grids & \multicolumn{2}{|c|}{$\overline{\text { FD-Method }}$} & \multicolumn{2}{|l|}{ Newton-MG } & \multicolumn{2}{|c|}{ FAS } \\
\hline$J$ & Itr & Time & Newton-Itr (linear-Itr) & Time & V-cycles & Time \\
\hline $11^{2}$ & 5 & 0.8846 & $4(4,5,3,1)$ & 0.0246 & 5 & 0.0100 \\
\hline $21^{2}$ & 6 & 2.7255 & $4(6,8,4,3)$ & 0.0524 & 5 & 0.0326 \\
\hline $41^{2}$ & 6 & 9.7324 & $5(5,8,6,5,4)$ & 0.2188 & 5 & 0.0818 \\
\hline $81^{2}$ & 6 & 23.0232 & $5(6,6,4,3,3)$ & 0.6784 & 6 & 0.2460 \\
\hline $161^{2}$ & 8 & 196.7221 & $5(6,6,5,5,6)$ & 7.2526 & 6 & 1.3182 \\
\hline $321^{2}$ & 8 & 266.3218 & $5(8,6,6,5,6)$ & 15.5473 & 5 & 3.9394 \\
\hline
\end{tabular}
(4.9) for $\lambda=1$ and for an increasing number of grid points.

Numerical difficulties occur when the second branch of the solution (the upper solution) needs to be computed particularly for small values of $\lambda$. In such a case, numerical methods 
are very sensitive with respect to the initial guess, especially for small values of $\lambda$ and when $\lambda$ approaches $\lambda_{c}$. Table 4.2 shows the comparison of three methods to get the upper solution of model (4.9) for $\lambda=1$ and for decreasing grid size $h$. Numerical results indicate that the convergence of the upper solution slows down with the increase of the number of grid points in FD-methods. Newton-MG with nested iteration needs to calculate the Jacobian matrix at each iteration. The convergence process also slows down when we increase the size of problem. In FAS-MG, we use a $V(2,2)$ cycle with MINRES as a relaxation smoother (both for pre and post relaxation).

Table 4.2: The number of iterations and the CPU time in seconds for the upper solution of model (4.9) for $\lambda=1$ for an increasing number of grid points.

\begin{tabular}{|c|c|c|c|c|c|c|}
\hline Grids & \multicolumn{2}{|c|}{ FD-Method } & \multicolumn{2}{|l|}{ Newton-MG } & \multicolumn{2}{|c|}{ FAS } \\
\hline$J$ & Itr. & Time & Newton-Itr (linear-Itr) & Time & V-cycles & Time \\
\hline $11^{2}$ & 11 & 2.3422 & $8(12,9,6,25,8,6,1,1)$ & 2.5606 & 32 & 0.2764 \\
\hline $21^{2}$ & 12 & 6.0568 & $8(23,12,28,7,4,5,2,2)$ & 5.3318 & 46 & 0.7564 \\
\hline $41^{2}$ & 11 & 8.7054 & $11(32,18,22,56,14,12,25,12,9,3,2)$ & 8.3275 & 35 & 0.9437 \\
\hline $81^{2}$ & 14 & 41.1728 & $11(42,63,56,24,36,24,46,11,6,1,1)$ & 16.1121 & 32 & 1.3693 \\
\hline $161^{2}$ & 28 & 448.0945 & $9(49,84,66,18,6,4,1,1,1)$ & 27.9396 & 32 & 6.9758 \\
\hline $321^{2}$ & 48 & 926.4628 & $11(33,68,56,32,21,15,13,11,5,3,3)$ & 41.8759 & 42 & 11.5745 \\
\hline
\end{tabular}

The difference between the performance of methods is more pronounced when we try to find the upper solution for small values of $\lambda$. Figure 4.2 shows the upper solution of model (4.9) obtained when we employ the FAS-MG method for small values of $\lambda$ on a grid of $161 \times 161$ grid points. For small values of $\lambda$, Table 4.3 demonstrates the convergence to the upper solution
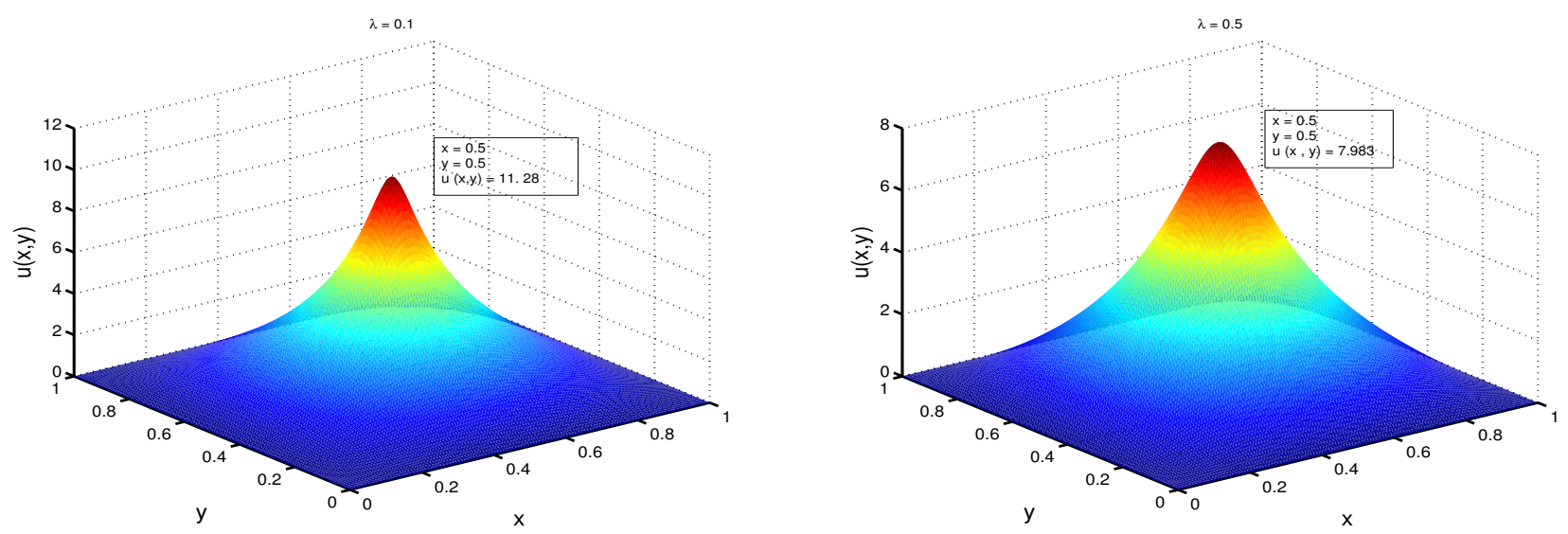

Figure 4.2: The upper solution of model (4.9) for small values of $\lambda$ on $161 \times 161$ grid points. For $\lambda=0.1$ (left) and $\lambda=0.5$ (right): FAS-MG-V $(2,2)$ was employed to obtain the upper solutions.

and provides a comparison of the efficiency of the three methods for $321 \times 321$ grid points. It is observed that for small grid sizes and especially for small values of $\lambda$, the convergence of the upper solutions becomes very slow and this affects the efficiency for both Newton-MG and FD methods. Particularly, for FAS-MG the convergence is slower, but the rate of slowing down is not very high. This shows the effectiveness of the FAS-MG method over the other two methods.

The convergence of the numerical solutions, especially for the upper solution, is of great importance. In earlier numerical reports, it has always been hard, or even impossible, to find 
Table 4.3: The number of iterations and the CPU time in seconds for the upper solution of model (4.9) for small values of $\lambda$ on $321 \times 321$ grid points.

\begin{tabular}{|c|c|c|c|c|c|c|}
\hline & \multicolumn{2}{|c|}{ FD-Method } & \multicolumn{2}{|l|}{ Newton-MG } & \multicolumn{2}{|c|}{ FAS } \\
\hline$\lambda$ & Itr & Time & Newton-Itr (linear-Itr) & Time & V-cycles & Time \\
\hline 0.1 & 63 & 1123.3865 & $13(74,46,22,48,92,76,18,36,42,12,8,5,5)$ & 211.9268 & 46 & 84.7324 \\
\hline 0.3 & 48 & 838.7255 & $11(85,33,76,36,62,25,12,28,8,12,1)$ & 152.1488 & 91 & 41.2247 \\
\hline 0.5 & 52 & 774.1730 & $9(42,38,82,25,62,34,15,8,6)$ & 112.5773 & 42 & 18.8102 \\
\hline$\lambda_{c}$ & 12 & 376.6732 & $12(50,23,38,32,18,12,15,7,5,3,3,2)$ & 42.7634 & 34 & 8.2155 \\
\hline
\end{tabular}

convergence to the second branch in the 2D Bratu problem (4.9) for $h \downarrow 0$. We investigated numerically the convergence behaviour for the upper solution when applying the three described methods. Figure 4.3 illustrates nicely the numerical convergence for nonlinear BVP (4.9) by using the proposed numerical approach. The numerical experiments demonstrate that the proposed methods converge rapidly, for both the lower and the upper solutions. These results yield better accuracy and are more efficient than previously reported numerical schemes, showing the effectiveness of proposed method.

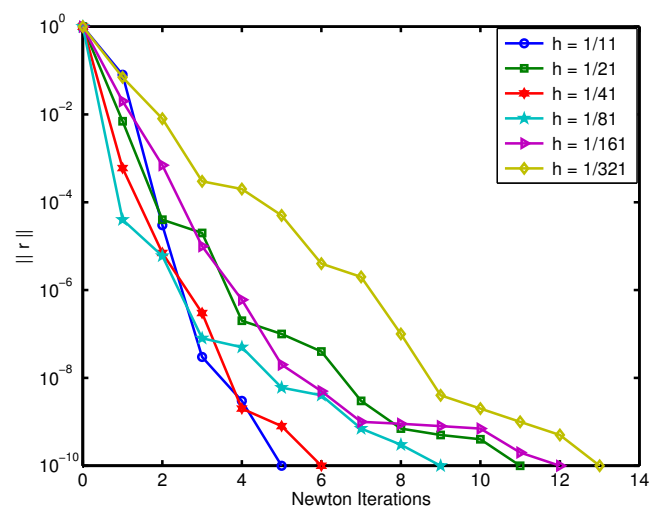

Convergence for nested Newton-MG for $\lambda=0.1$

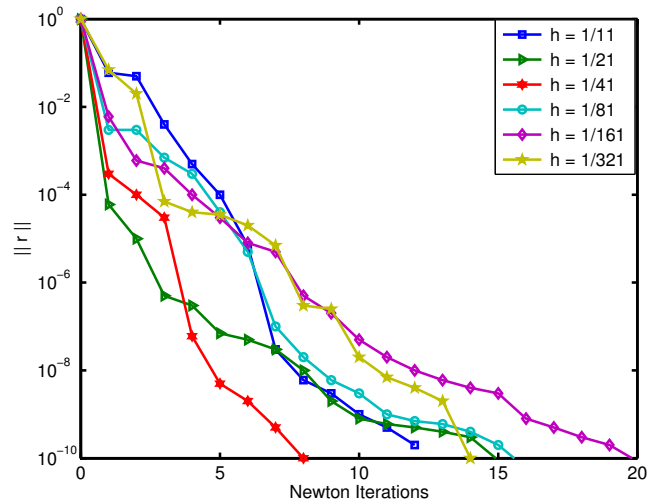

Convergence for nested Newton-MG for $\lambda=\lambda_{c}$

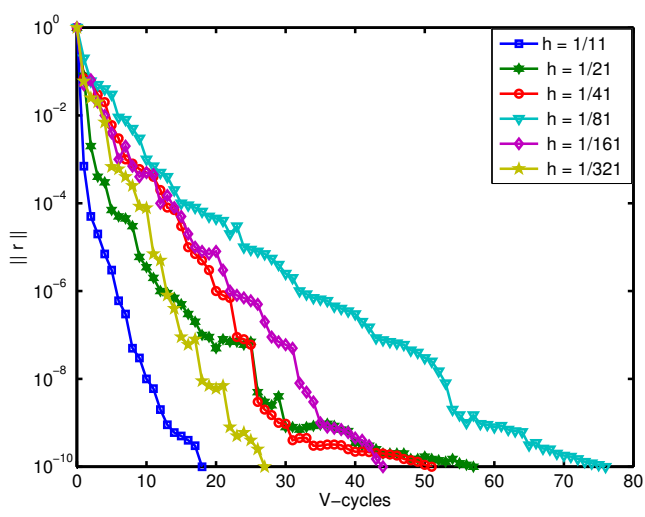

Convergence for FAS-MG for $\lambda=0.1$

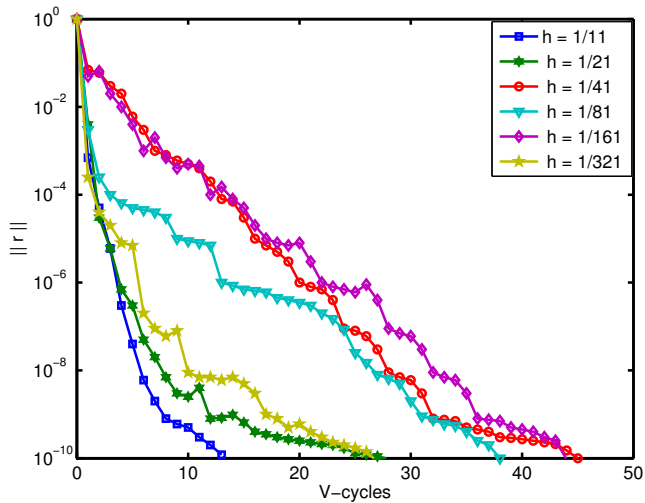

Convergence for FAS-MG for $\lambda=\lambda_{c}$

Figure 4.3: The numerical convergence for the upper solution for $\lambda=0.1$ and the unique solution for $\lambda=\lambda_{c}$ of problem (4.9) on decreasing grid sizes $h$ using the nested Newton-MG method (left two figures) and the FAS-MG-V $(2,2)$ method (right two figures). 
Numerical results clearly indicate that FAS-MG is more efficient than the Newton-MG and the FD method for computing both positive solutions (lower and upper) of BVP (4.9) for $\lambda \epsilon$ $\left(0, \lambda_{c}\right]$. The numerical bifurcation diagram is presented in Figure 4.4 of the two-dimensional Bratu model (4.9), using the FAS-MG on a grid of $161 \times 161$ grid points. The upper and lower solutions are presented by red and blue lines, respectively. It is observed that both onedimensional and two-dimensional GB models depict similar bifurcation behaviour.

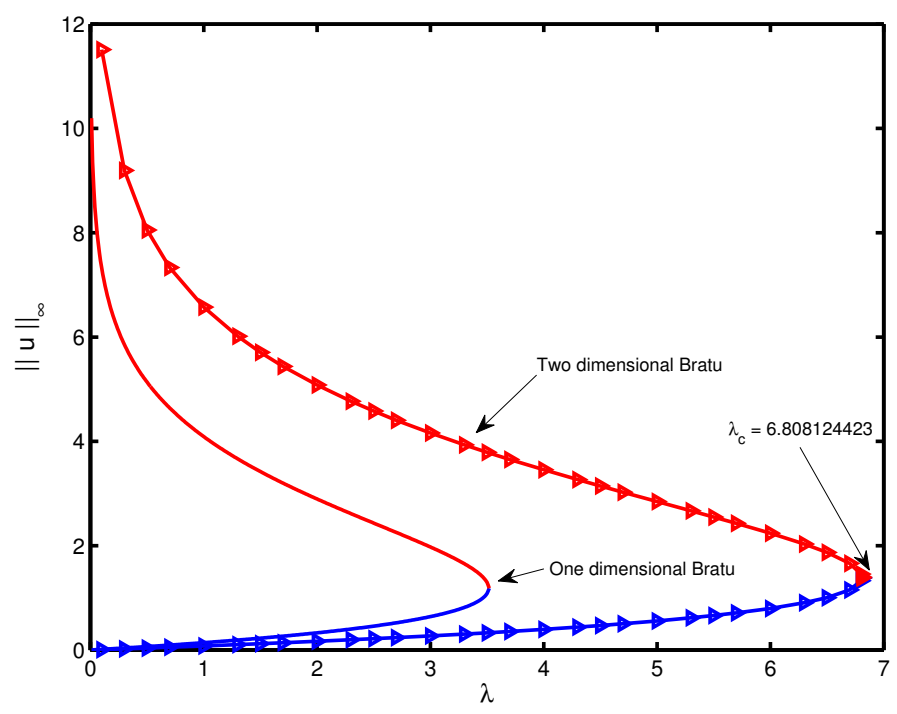

Figure 4.4: The bifurcation diagram of the two-dimensional Bratu-model (4.9) using the FASMG-MINRES approach on $161 \times 161$ grid points, compared to the $1 \mathrm{D}$ case (see also Chapter 2 and [148]). The critical value $\lambda_{c} \approx 6.808124423$.

It has been reported in [148] for the one-dimensional (1D) Bratu model case, that new types of solutions (periodic and semi-periodic) exist by considering a Taylor approximation of the nonlinear term $\mathrm{e}^{u}$. Motivated by the 1D case in Chapter 2 and [148], we extend the numerical results for the 2D Bratu problem. For this, we take the Taylor approximation of $\mathrm{e}^{u}$ up to quadratic and cubic terms of problem (4.9) and present new types of solutions. Bifurcation diagrams are presented in the experiments 2 and 3 in the next sections.

\subsubsection{Experiment 2: a quadratic approximation}

We consider a Taylor approximation of $\mathrm{e}^{u}$ up to the quadratic term. Then model (4.9) with Dirichlet boundary conditions can be written as:

$$
\begin{gathered}
\Delta u+\lambda\left(1+u+\frac{1}{2} u^{2}\right)=0, \quad(x, y) \in[0,1]^{2}, \\
\left.u\right|_{\partial \Omega}=0 .
\end{gathered}
$$

The solution behaviour of model (4.10) resembles that of the classical Bratu problem (4.9) in the sense that two, one or zero solutions exist for $\lambda<\lambda_{c}, \lambda=\lambda_{c}$ or $\lambda>\lambda_{c}$, respectively. For this case the critical value $\lambda_{c} \approx 7.813521632$ is computed by using Newton's method. Tables 4.4 and 4.5 summarize the performance of the described methods when employed to obtain the solutions for model (4.10). It can be observed that for small values of $\lambda$, the proposed methods converge to both solutions (lower and upper) successfully on a grid of $321 \times 321$ grid points. Numerical results indicate that the performance of the proposed methods for model (4.10) is better than 
for problem (4.9) as $f(u)$ is not a highly nonlinear function in model (4.10). Further it is noted that FAS-MG is found to be more efficient than nested Newton-MG and the FD-method with respect to computational time.

Table 4.4: The number of iterations and the CPU time in seconds for the lower solution of model(4.10) for small values of $\lambda$ on $321 \times 321$ grid points.

\begin{tabular}{|c||c||c||c||c||c||c|}
\hline \hline \multicolumn{1}{|c||}{} & \multicolumn{2}{c||}{ FD-Method } & \multicolumn{2}{c||}{ Newton-MG } & \multicolumn{2}{c|}{ FAS } \\
\hline$\lambda$ & Itr & Time & Newton-Itr & Time & V-cycles & Time \\
\hline \hline 0.1 & 13 & 196.7281 & $6(16,22,6,15,3,3)$ & 103.04831 & 12 & 34.7122 \\
0.2 & 11 & 166.2892 & $5(12,18,8,4,4)$ & 78.2824 & 8 & 21.9314 \\
0.3 & 11 & 142.7429 & $6(22,15,18,8,2,5)$ & 62.2669 & 10 & 19.0612 \\
0.5 & 15 & 129.2432 & $6(8,6,12,6,3,3)$ & 40.8436 & 8 & 15.0946 \\
1 & 13 & 111.8652 & $5(8,13,5,5,4)$ & 15.7763 & 8 & 6.1644 \\
\hline
\end{tabular}

Table 4.5: The number of iterations and the CPU time in seconds for the upper solution of problem (4.10) for small values of $\lambda$ on $321 \times 321$ grid points.

\begin{tabular}{|c|c|c|c|c|c|c|}
\hline & \multicolumn{2}{|c|}{ FD-Method } & \multicolumn{2}{|l|}{ Newton-MG } & \multicolumn{2}{|c|}{ FAS } \\
\hline$\lambda$ & $\operatorname{Itr}$ & Time & Newton-Itr (linear-Itr) & Time & V-cycles & Time \\
\hline 0.1 & 62 & 867.8210 & $11(72,96,65,84,58,37,18,5,7,9,3)$ & 148.3319 & $\overline{92}$ & 39.7621 \\
\hline 0.2 & 60 & 834.2298 & $9(92,56,52,88,44,15,9,5,6)$ & 124.0352 & 68 & 34.2986 \\
\hline 0.3 & 66 & 844.3205 & $8(46,82,96,38,42,18,5,1)$ & 96.3809 & 84 & 29.3153 \\
\hline 0.5 & 45 & 633.9921 & $12(65,54,90,36,28,15,24,15,9,5,7,5)$ & 74.3112 & 48 & 15.0037 \\
\hline 1 & 48 & 624.3172 & $8(48,67,32,16,9,12,5,1)$ & 42.4507 & 42 & 9.1496 \\
\hline
\end{tabular}

In addition to the above mentioned solutions, we also provide the numerical convergence for the unique solutions of model (4.10) for the critical value $\lambda_{c}$. The convergence to the unique solution is obtained by using both Newton-MG and FAS-MG methods for decreasing values of $h$, see Figure 4.5 .
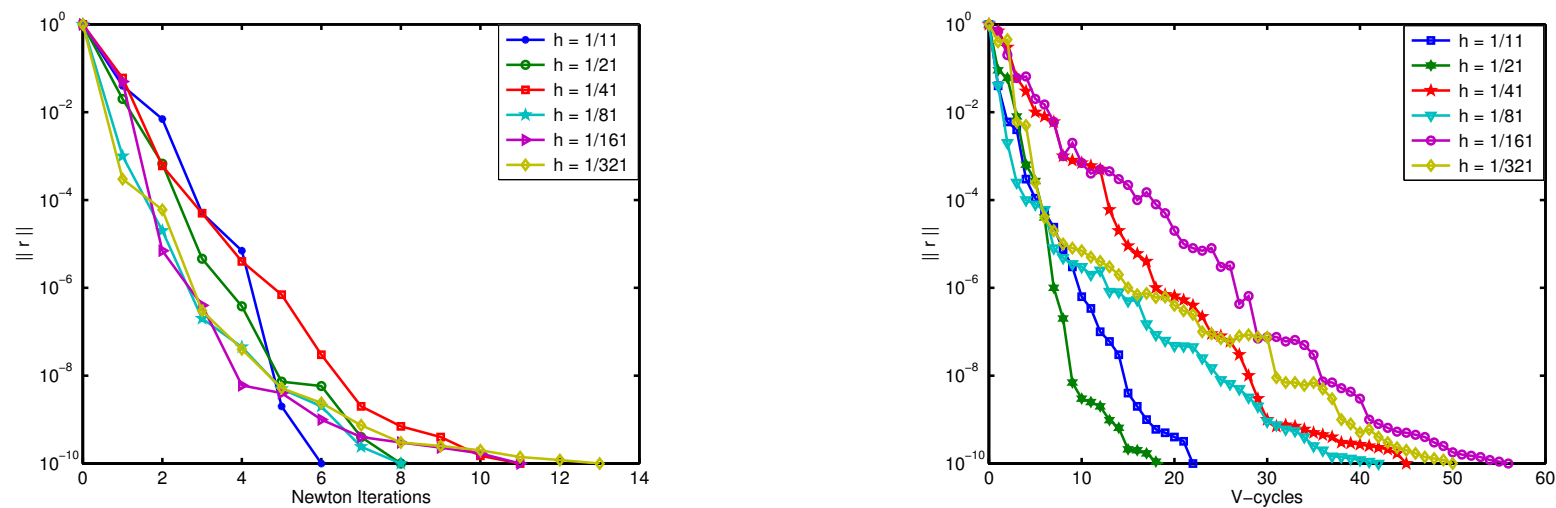

Figure 4.5: Numerical convergence for the unique solution of model (4.10) for $\lambda=\lambda_{c}$ using nested Newton-MG (left) and FAS-MG- $V(2,2)$ (right) for decreasing values of the grid sizes.

Furthermore, we investigate the numerical bifurcation behaviour of model (4.10) for different values of $\lambda \in\left(0, \lambda_{c}\right]$. The numerical bifurcation diagram is presented in Figure 4.6, using the 
FAS-MG on $161 \times 161$ grid points. The upper and lower solutions are presented by red (dash) and blue (solid) curves respectively. It is observed that despite having different critical values for two-dimensional nonlinear models (4.9) and (4.10), both depict the same bifurcation behaviour (see Figures 4.4 and 4.6). Moreover, like in the 1D case [148], a similar bifurcation behaviour can be observed by approximating the nonlinear term $\mathrm{e}^{u}$ up to even-power approximations.

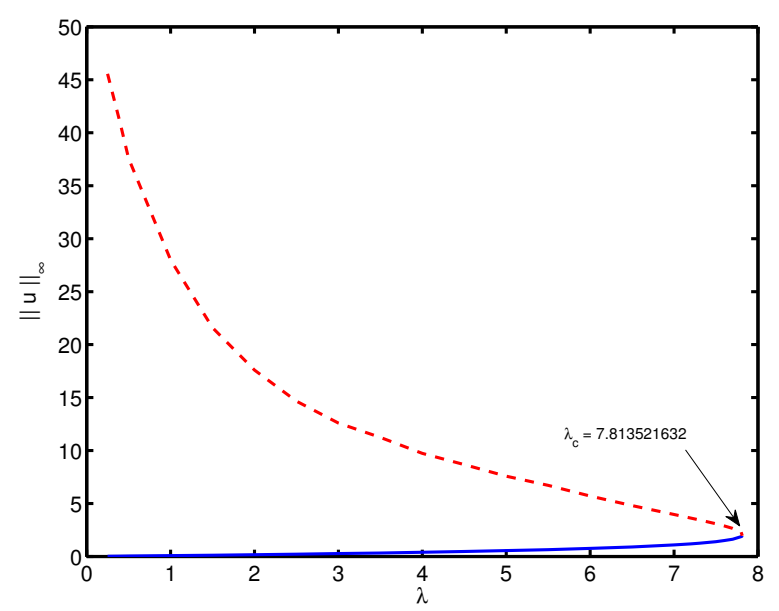

Figure 4.6: The bifurcation curve for problem (4.10) is obtained by using FAS-MG on $161 \times 161$ grid points with the critical value $\lambda_{c} \approx 7.813521632$. The solid line (blue) denotes the lower solution and the dashed line (red) the upper solution, respectively.

\subsubsection{Experiment 3: a cubic approximation}

Next, we approximate the nonlinear term of $\mathrm{e}^{u}$ up to the cubic term:

$$
\begin{aligned}
& \Delta u+\lambda\left(1+u+\frac{1}{2} u^{2}+\frac{1}{6} u^{3}\right)=0, \quad(x, y) \in \Omega=[0,1]^{2}, \\
& \left.u\right|_{\partial \Omega}=0 .
\end{aligned}
$$

This model also posseses the same properties as described for models (4.9) and (4.10), when considering positive solutions only. We computed the critical value $\lambda_{c} \approx 7.011421641$ by using Newton's method. Numerical results for model (4.11) are presented in Tables 4.6 and 4.7 for different small values of $\lambda$ on a $321 \times 321$ grid. Numerical convergence for the critical value $\lambda_{c}$ is shown in Figure 4.8. As for experiment 2, it is again observed that FAS-MG is more efficient than the nested Newton-MG method and the FD-method with respect to computational time. These results demonstrate the validity and applicability of the FAS-MG method as presented in section 4.2 .

The numerical bifurcation curve for positive solutions, shows two solutions (lower and upper) and a unique solution using the FAS-MG method for $\lambda \in\left(0, \lambda_{c}\right]$ (see Figure 4.7).

\section{Periodic and semi-periodic solutions}

In chapter 2, [148], we found that, for this cubic approximation, more than two solutions may exist. These solutions can be either periodic or semi-periodic and the number of solutions varies from $0,1,2$ up to $\infty$. We define these periodic and semi-periodic solutions in the following way: 


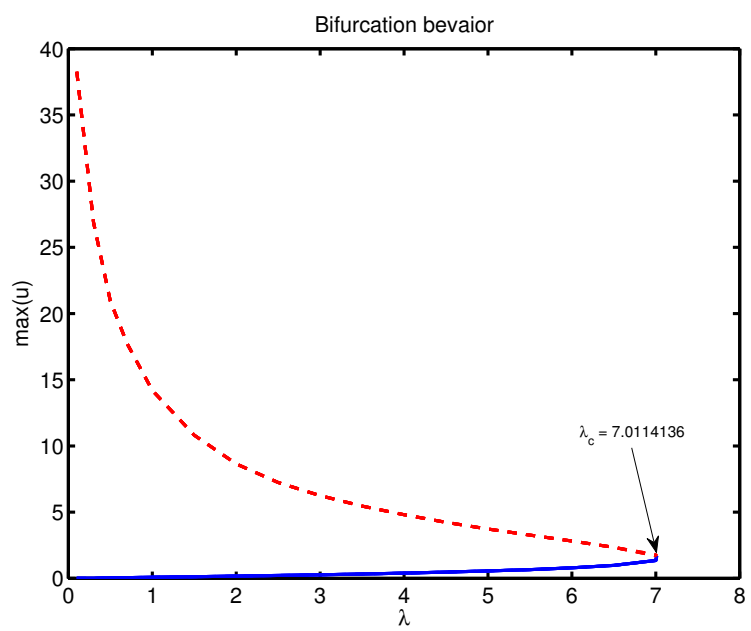

Figure 4.7: The bifurcation curve for model (4.11) is obtained by employing FAS-MG (for positive solutions only) for different values of $\lambda$. Red (dashed line) and blue (solid line) show the upper and lower solutions, respectively. For model (4.11) the critical value $\lambda_{c} \approx 7.011421641$.

Table 4.6: The number of iterations and the CPU time in seconds for the lowest solution of problem (4.11) for small values of $\lambda$.

\begin{tabular}{|c||c||c||c|c||c||c|}
\hline \hline \multicolumn{1}{|c||}{} & \multicolumn{2}{c||}{ FD-Method } & \multicolumn{2}{c||}{ Newton-MG } & \multicolumn{2}{c|}{ FAS } \\
\hline$\lambda$ & Itr & Time & Newton -Itr (linear Itr) & Time & V-cycles & Time \\
\hline \hline 0.1 & 15 & 214.8177 & $8(22,18,12,15,6,4,8,3)$ & 116.2213 & 12 & 42.1348 \\
0.2 & 13 & 171.0430 & $6(18,26,22,14,5,7)$ & 94.3319 & 14 & 22.8256 \\
0.3 & 11 & 146.2219 & $8(15,22,18,11,9,5,5,1)$ & 66.1428 & 12 & 19.8403 \\
0.5 & 11 & 134.2301 & $5(11,15,9,5,2)$ & 43.9287 & 12 & 15.2298 \\
1 & 9 & 118.0972 & $6(11,9,8,5,1,1)$ & 18.0525 & 8 & 6.6635 \\
\hline
\end{tabular}

Table 4.7: The number of iterations and the CPU time in seconds for the upper solution of problem (4.11) for small values of $\lambda$.

\begin{tabular}{|c|c|c|c|c|c|c|}
\hline & \multicolumn{2}{|c|}{ FD-Method } & \multicolumn{2}{|l|}{ Newton-MG } & \multicolumn{2}{|c|}{ FAS } \\
\hline$\lambda$ & Itr & Time & Newton-Itr (linear Itr) & Time & V-cycles & Time \\
\hline 0.1 & 72 & 894.1275 & $13(56,62,48,35,33,30,44,20,15,11,11,9,2)$ & 151.7743 & 86 & 42.2361 \\
\hline 0.2 & 56 & 826.3816 & $11(66,75,18,22,26,36,9,5,3,3,1)$ & 126.2758 & 68 & 37.1184 \\
\hline 0.3 & 68 & 719.0528 & $11(46,36,22,20,34,18,12,11,5,7,3)$ & 108.0543 & 84 & 35.2846 \\
\hline 0.5 & 56 & 664.1194 & $12(36,42,35,18,12,9,9,9,12,5,1,1)$ & 81.3286 & 48 & 21.8137 \\
\hline 1 & 44 & 641.0037 & $11(46,54,22,34,12,12,8,6,3,7,5)$ & 49.3371 & 36 & 14.7414 \\
\hline
\end{tabular}



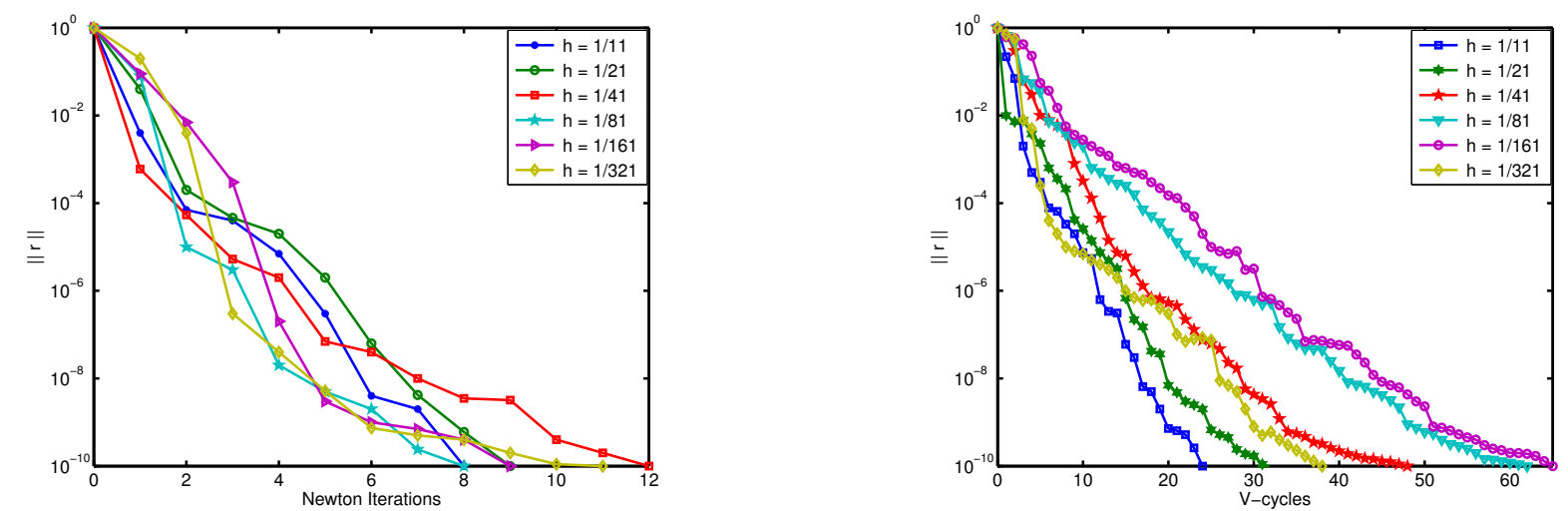

Figure 4.8: Numerical convergence by using nested Newton-MG (left) and FAS-MG (right) for the unique solution at different grid sizes of model (4.11) for $\lambda=\lambda_{c}$ ).

$$
(l, m)= \begin{cases}\text { Periodic } & ; \& m=2,4,6,8, \ldots, \\ \text { Semi-periodic } \quad ; \quad \text { otherwise }\end{cases}
$$

where $l$ and $m$ are the number of extrema along the horizontal and vertical straight lines respectively in solution $u(x, y)$. For example, $(2,3)$ represents a semi-periodic solution, referring to the definition in (4.12). It indicates that the solution $u(x, y)$ has $l=3$ extrema along the horizontal axis and $m=2$ extrema along the vertical axis. Similarly, $(3,3)$ represents a semi-periodic solution, whereas $(4,2)$ and $(6,6)$ represent a periodic solution as $l$ and $m$ are even numbers. To find this new multiplicity of solutions, we employed the FAS-MG method which is found as more efficient than the other two methods (see experiment 1 and 2). Both the periodic and semi-periodic solutions are obtained successfully with the $V(2,2)$-cycle in FAS-MG for $\lambda=1$ on $161 \times 161$ grid points. Some of the periodic and semi-periodic solutions and their corresponding contour plots for $\lambda=1$ are presented in Figure 4.9 and Figure 4.10, respectively. Furthermore, it is important to note that the numerical convergence for these solutions is achieved successfully. The numerical convergence for the case $(2,2)$ periodic solution and for $\lambda=1$ as a function of the grid size $h$ is depicted in Figure 4.11. These results again confirm the effectiveness of the FAS-MG method. However, for decreasing grid sizes, FAS-MG could not obtain the desired accuracy. Moreover, a similar bifurcation behaviour (for positive solutions only) can be obtained by approximating the nonlinear term $\mathrm{e}^{u}$ up to odd power approximations like in the 1D case [148.

The three numerical experiments as discussed above, clearly show that the proposed methods, namely, a FD based method, Newton-MG and FAS-MG converge successfully for the twodimensional GB model for small values of $\lambda$ on an increasing number of grid points. However, the efficiency in terms of computational time of FAS-MG is better than for the other two methods. Furthermore, FAS-MG is used successfully to obtain the convergence of new multiple solutions with greater accuracy and efficiency. Both FD and Newton-MG methods lose efficiency and desired accuracy for decreasing grid sizes when trying to compute new multiple solutions. This shows the effectiveness of FAS-MG. 


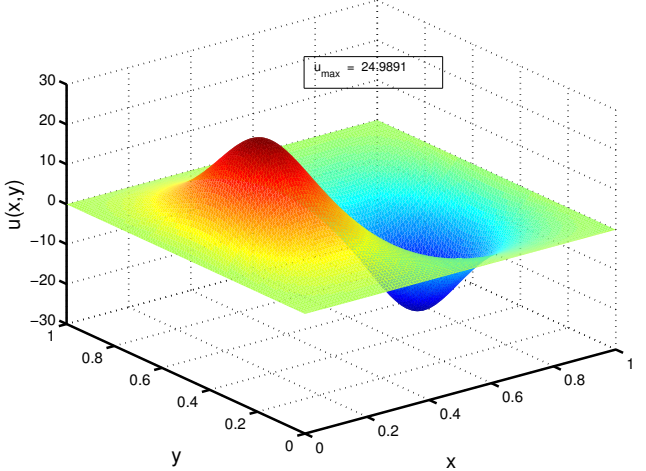

Semi-periodic

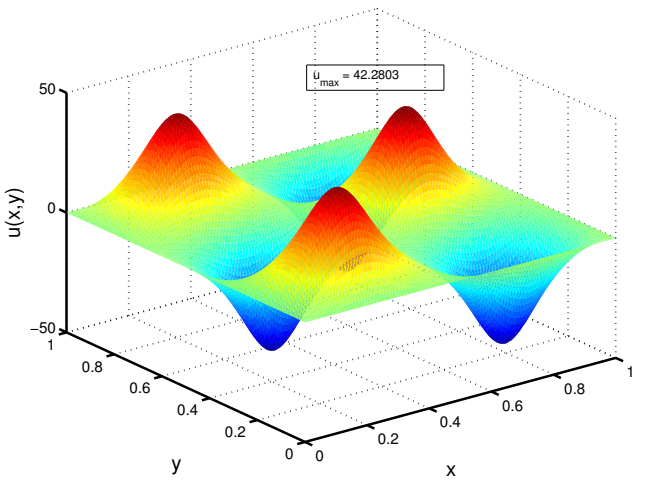

Semi-periodic

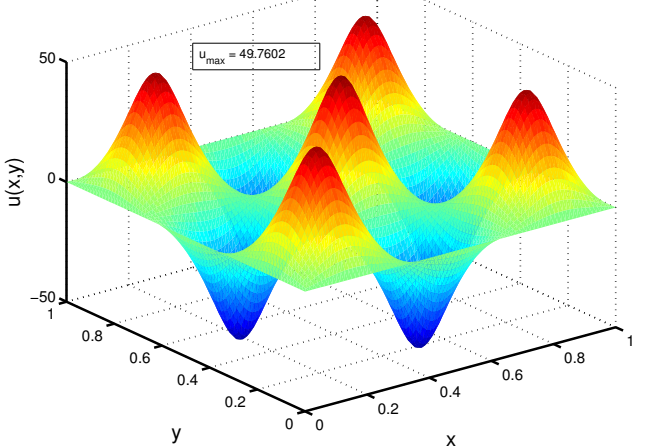

Semi-periodic

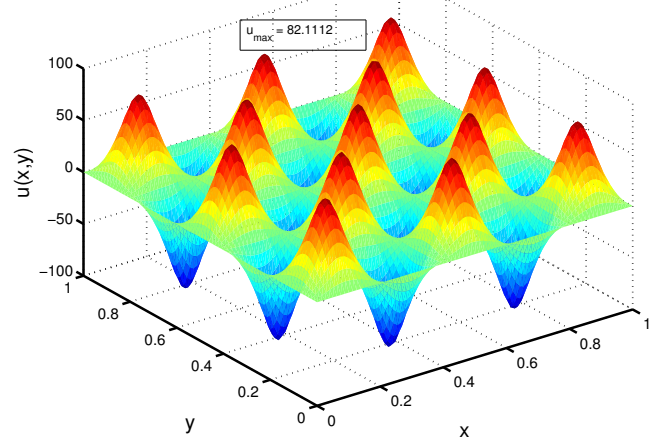

Semi-periodic

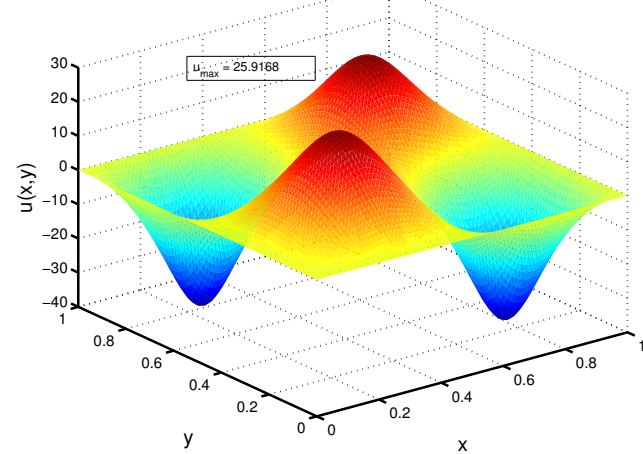

Periodic

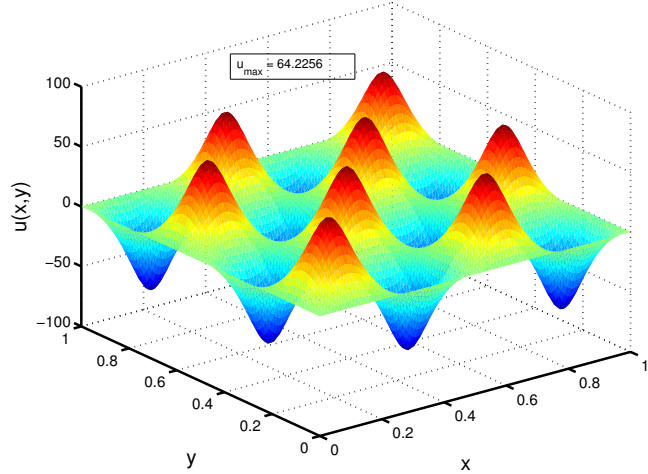

Periodic

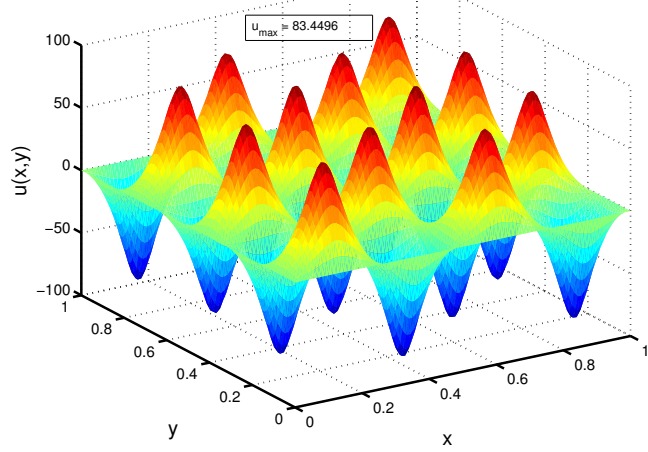

Periodic

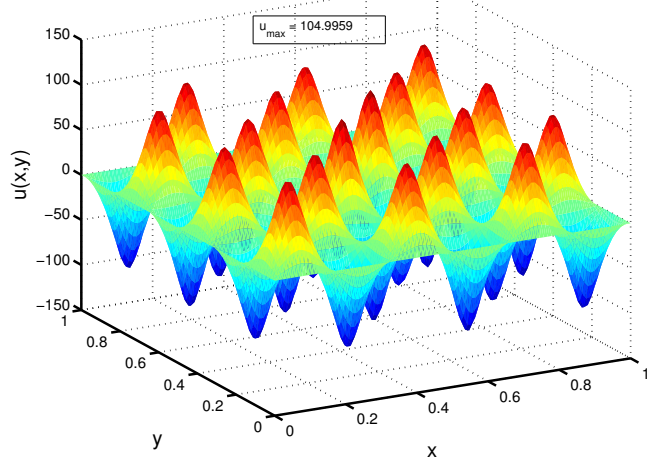

Periodic

Figure 4.9: Some periodic and semi-periodic solutions for $\lambda=1$ of model (4.11) obtained by using FAS-MG with $101 \times 101$ grid points. 


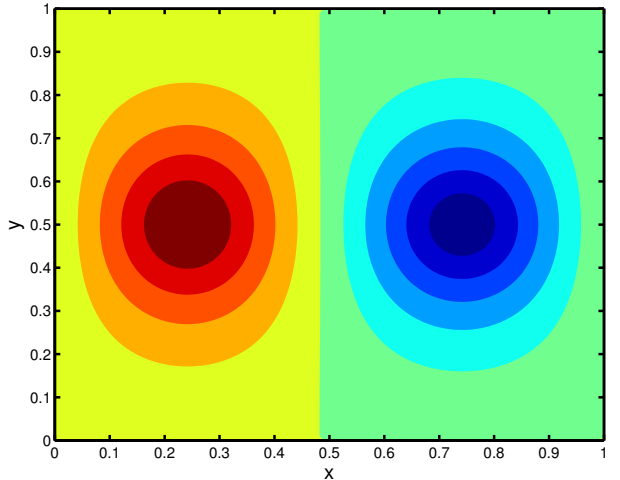

$(2,1)$

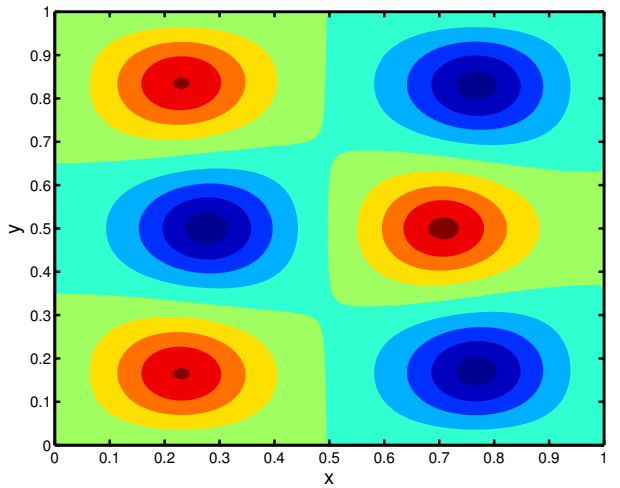

$(2,3)$

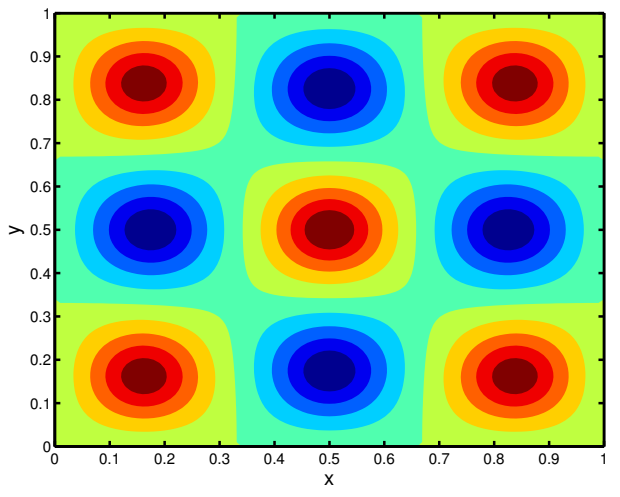

$(3,3)$

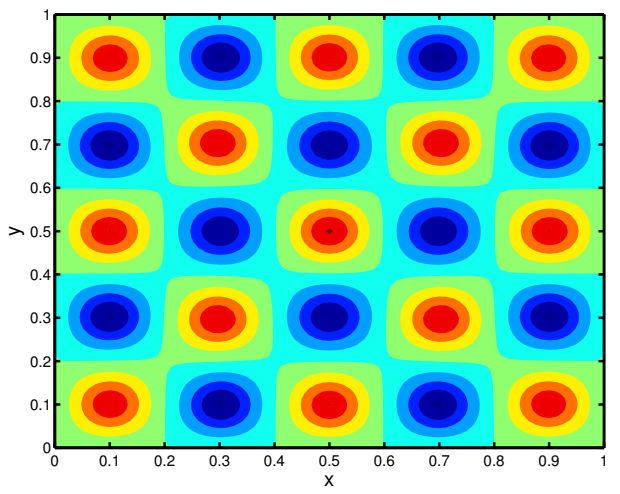

$(5,5)$

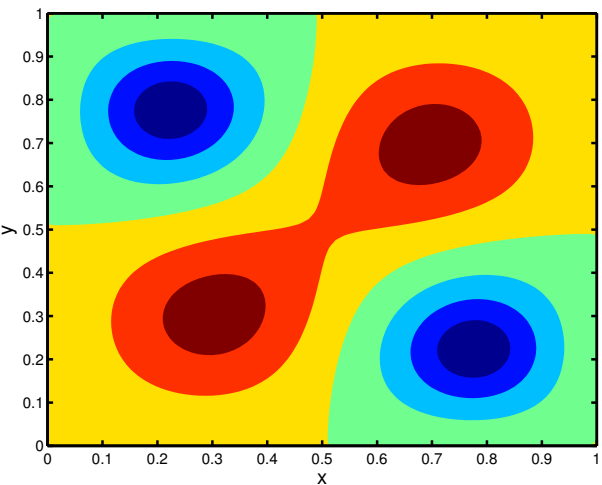

$(2,2)$

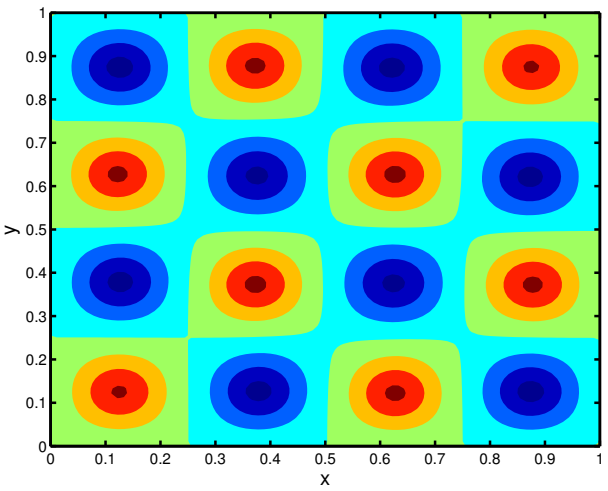

$(4,4)$

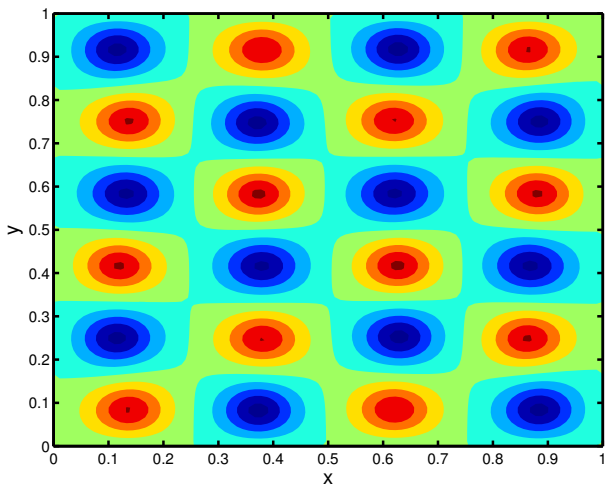

$(4,6)$

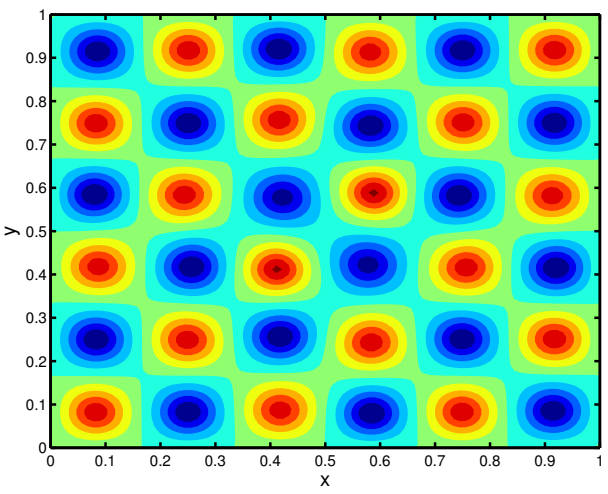

$(6,6)$

Figure 4.10: The corresponding contour plots of periodic and semi-periodic solutions of Figure 4.9 for $\lambda=1$ of model (4.11). 

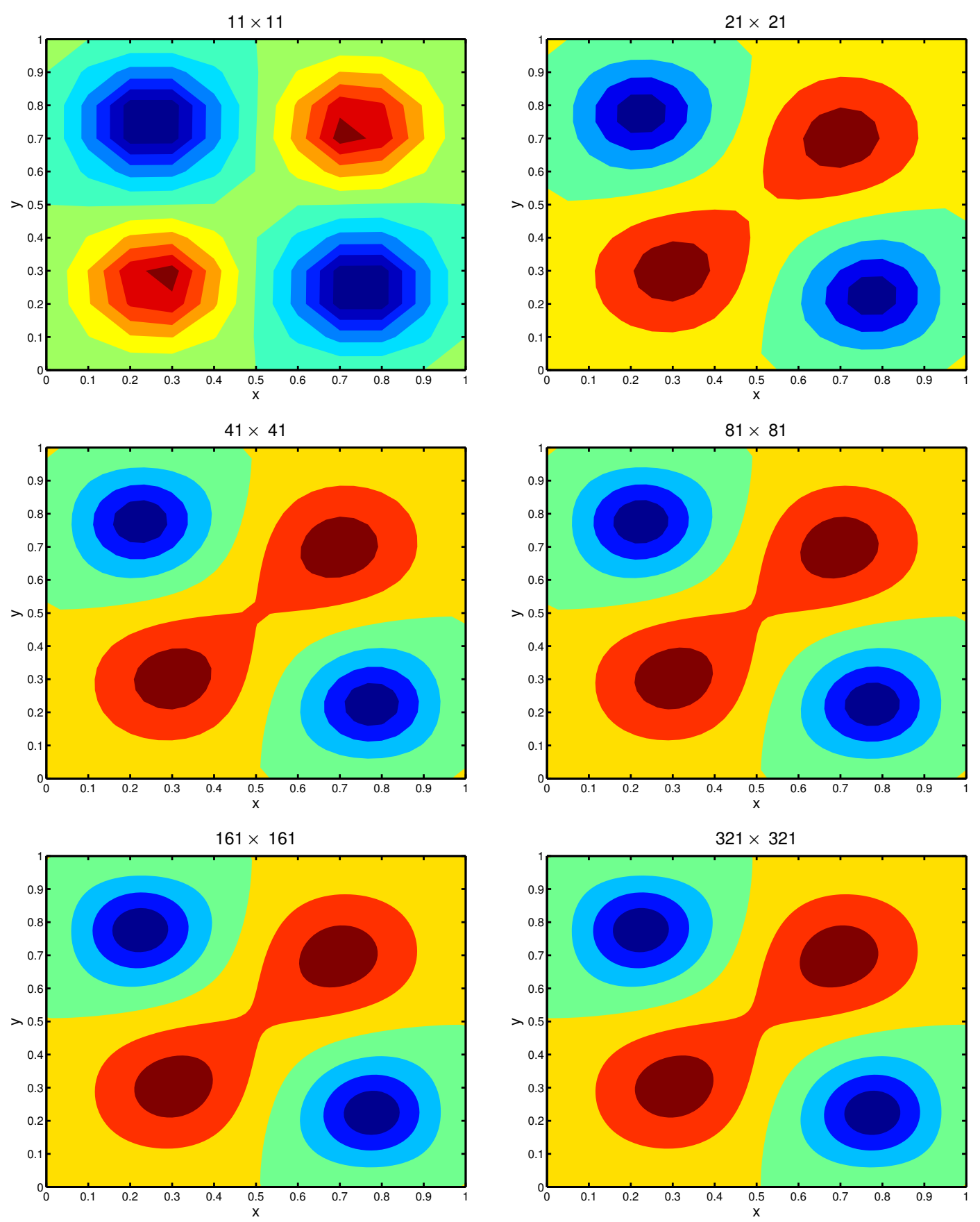

Figure 4.11: Contour plots of the numerical convergence in the case of the $(2,2)$ periodic solution on an increasing number of grid points for $\lambda=1 \mathrm{in}$ model (4.11). The results are obtained by using the FAS-MG-V(2,2) method. 


\subsection{Conclusions}

In this chapter, we have numerically computed the two-dimensional Bratu-type nonlinear boundary-value problems. We proposed three numerical methods, viz, a finite-difference based method with fsolve and the two MG approaches: Newton-MG and FAS-MG with the MINRES method as relaxation smoother. With these methods we have been able to overcome the convergence difficulties without compromising the efficiency. A comparison, in terms of convergence, accuracy and efficiency between the three numerical methods demonstrated an improvement for the whole parameter range $\lambda \in\left(0, \lambda_{c}\right]$. Furthermore, we investigated the bifurcation behaviour of solutions for the two-dimensional Bratu models and found new multiplicity of solutions in the case of cubic approximation of the nonlinear exponential term. It has been demonstrated successfully that the convergence of all solutions namely, unique, lower, upper, periodic and semi-periodic has been obtained for small values of the parameter $\lambda$. FAS-MG is found to be more efficient than the other two methods as observed in numerical results. This success is achieved due to an appropriate initial guess and with the use of the MINRES method as relaxation smoother. 


\section{Chapter 5}

\section{A numerical study of the higher-dimensional Gelfand-Bratu model}

\subsection{Introduction}

In the present chapter, we consider a higher-dimensional nonlinear elliptic boundary-value problem: the Gelfand Bratu (GB) model [42, 17, 18] which depends heavily on the parameter $\lambda$. This model coupled with appropriate boundary conditions is used to model the temperature distribution in fuel ignition problems [11]. The GB model simulates also a thermal reaction process in a rigid material where the process depends on the balance between chemically generated heat and heat transfer by conduction [139, 24]. The three-dimensional model is considered in investigations on the sun core temperatures [23, 24]. The GB model appears in several contexts such as thermo-electro-hydrodynamics models [137] and elasticity theory [120].

In literature, only a few numerical methods have been proposed for the GB model in higher dimensions. For the three-dimensional GB model, only two solutions (lower and upper) were presented by using a nonstandard compact finite difference scheme in [51]. A pseudospectral method, finite difference method and radial basis functions method for the three-dimensional GB problem, are discussed in [66] which illustrates the bifurcation behaviour in detail. On the unit ball, the GB model is investigated numerically in which the solution dependence on the parameter $\lambda$ for all dimension parameters $n$ is described in [64] and radial symmetry is demonstrated in [43]. Three-dimensional bifurcation diagrams on a ball and annular domains are discussed with the help of a pseudo-arclength continuum method in [83].

In this work, we show numerical convergence to different types of solutions and present a bifurcation curve for the GB model in higher dimensions. We focus our research on positive solutions for $\lambda>0$. For the numerical computation of the three-dimensional GB model, we have chosen to use a nonlinear multigrid approach. This multigrid approach can be applied directly to the nonlinear elliptic equations without the use of a global linearization technique. This is known as full approximation storage (FAS). In FAS multigrid, linearization of the nonlinear problem is treated locally in the relaxation step on different grid sizes. It is well known that the FAS multigrid idea is based on two principles: an error smoothing on fine grids and followed by coarse grid corrections. In this work, we concentrate on the computational difficulties such as a possible unstable convergence behaviour and the loss of diagonal dominance of the Jacobian matrix for

This chapter is based on an article in press as [59]: S. Iqbal and P. A. Zegeling, Journal of Computers and Mathematics with Applications, 2020. 
obtaining the upper branches of solutions of the GB model by the FAS multigrid approach. The Jacobian matrix depends on the solution values, the parameter $\lambda$ and the grid size $h$. To handle these difficulties, we use a MINRES method as a smoother in FAS multigrid approach instead of a classical smoother (Gauss-Seidel method). This multigrid approach solves successfully the problems of the Jacobian matrix for large values of the solutions, which correspond to the upper solution branches. The main contribution of the present work in the three-dimensional GB model, is to compute accurately multiple solutions and to confirm the numerical convergence of the upper branches of solutions for all values of $\lambda \in\left(0, \lambda_{c}\right]$. Furthermore, we investigate the numerical bifurcation behaviour of the GB problem in three dimensions and identify the existence of two new turning points. As known from the literature [83, 66], finding multiple solutions and new turning points for higher dimensions in the GB problem is a hard task. Our numerical results illustrate the accuracy and efficiency of the proposed multigrid approach.

For even higher space dimensions (greater than three), we transform the GB problem using $n$-dimensional spherical coordinates to a nonlinear ordinary differential equation (ODE). The numerical solutions of this nonlinear ODE are computed by a shooting method for a range of values of the dimension parameter $n$. We compute several types of solutions of the GB model on an $n$-dimensional ball $B$ for different values of $n$ and $\lambda$. All multiple solutions of the nonlinear ODE on the ball $B$ can be proved to be positive, because of the maximum principle and radial symmetry (for more details, see [11]). In the present work, we numerically confirm the existence of multiple solutions depending on the parameters $n$ and $\lambda$. Numerical results clarify the bifurcation curves in the higher-dimensional GB problem obtained from theoretical results in literature [11].

This chapter is organized as follows. In section 5.2, we describe the GB problem in $n$ space dimensions. Section 5.3 is devoted to the discretization of the $n$-dimensional Laplacian. In section 5.4, the three-dimensional GB model is discussed, where in the nonlinear multigrid approach FAS is used to compute the numerical results and illustrate the multiplicity of solutions in detail. The GB model for higher dimensions is discussed in section 5.5, which incorporate a coordinate transformation from Cartesian to $n$-dimensional spherical coordinates to obtain a nonlinear ODE on a unit ball $B \subset \mathbb{R}^{n}$. Numerical experiments show several types of solutions and create bifurcation diagrams. The concluding remarks are presented in section 5.6.

\subsection{A boundary-value problem in $n$ space dimensions}

Consider the following nonlinear elliptic boundary-value problem, also known as the $n$ dimensional GB model:

$$
\begin{array}{rr}
\Delta u(\overrightarrow{\mathrm{x}})+\lambda \mathrm{e}^{u(\overrightarrow{\mathrm{x}})}=0, & \mathrm{x} \in \Omega=[0,1]^{n} \subset \mathbb{R}^{n}, \\
u(\overrightarrow{\mathrm{x}})=0, & \overrightarrow{\mathrm{x}} \in \partial \Omega,
\end{array}
$$

where $\lambda>0, \overrightarrow{\mathrm{x}}=\left(x_{1}, x_{2}, x_{3}, \ldots, x_{n}\right)^{T}$ and the Laplacian operator $\Delta$ in $n$ dimensions is given by:

$$
\Delta=\sum_{k=1}^{n} \frac{\partial^{2}}{\partial x_{k}^{2}} .
$$

\subsection{Discretization of the Laplacian in $n$ space dimensions}

For the numerical solution of model (5.1), we work out a central finite difference scheme for the $n$-dimensional Laplacian. On a uniform grid on the domain $\Omega$, we generate the grid points by 


$$
\left(\mathrm{x}_{i}\right)_{j}=j h, \quad \text { with } \quad h=\frac{1}{J}, \quad i=1,2, \ldots, n, \quad j=0,1,2, \ldots, J .
$$

We write $u_{i_{1}, i_{2}, i_{3}, \ldots, i_{n}}$ to represent the discrete approximation of the exact (unknowns) values $u\left(x_{1}, x_{2}, x_{3}, \ldots, x_{n}\right)$. A second-order central finite difference approximation for dimensions $1,2, \ldots, n$ reads, respectively:

$$
\begin{aligned}
& 1 d:\left.\quad \Delta u\right|_{i_{1}}=\left.u_{x x}\right|_{i_{1}} \approx \frac{1}{h^{2}}\left(u_{i_{1}+1}+u_{i_{1}-1}-2 u_{i_{1}}\right) \\
& 2 d:\left.\Delta u\right|_{i_{1}, i_{2}}=\left.u_{x_{1} x_{1}}\right|_{i_{1}, i_{2}}+\left.u_{x_{2} x_{2}}\right|_{i_{1}, i_{2}} \approx \frac{1}{h^{2}}\left(u_{i_{1}+1, i_{2}}+u_{i_{1}-1, i_{2}}+u_{i_{1}, i_{2}+1}+u_{i_{1}, i_{2}-1}-4 u_{i_{1}, i_{2}}\right), \\
& 3 d:\left.\Delta u\right|_{i_{1}, i_{2}, i_{3}}=\left.u_{x_{1} x_{1}}\right|_{i_{1}, i_{2}, i_{3}}+\left.u_{x_{2} x_{2}}\right|_{i_{1}, i_{2}, i_{3}}+\left.u_{x_{3} x_{3}}\right|_{i_{1}, i_{2}, i_{3}} \\
& \approx \frac{1}{h^{2}}\left(u_{i_{1}+1, i_{2}, i_{3}}+u_{i_{1}-1, i_{2}, i_{3}}+u_{i_{1}, i_{2}+1, i_{3}}+u_{i_{1}, i_{2}-1, i_{3}}+u_{i_{1}, i_{2}, i_{3}+1}+u_{i_{1}, i_{2}, i_{3}-1}-6 u_{i_{1}, i_{2}, i_{3}}\right), \\
& n d:\left.\Delta u\right|_{i_{1}, i_{2}, i_{3} \ldots i_{n}} \approx \frac{1}{h^{2}} \underbrace{\left(u_{i_{1}+1, i_{2}, i_{3}, \ldots, i_{n}}+u_{i_{1}-1, i_{2}, i_{3}, \ldots, i_{n}}+\ldots+u_{i_{1}, i_{2}, i_{3}, \ldots, i_{n}+1}+u_{i_{1}, i_{2}, i_{3}, \ldots, i_{n}-1}\right.}_{2 n \text { terms }} \\
& \left.-2 n u_{i_{1}, i_{2}, i_{3}, \ldots, i_{n}}\right) \text {. }
\end{aligned}
$$

\subsection{The three-dimensional case}

In this section, we treat the three-dimensional $(3 \mathrm{D})$ case $(n=3)$. The main goal is to numerically determine the multiple existence of solutions for a given bifurcation parameter $\lambda>0$ on the domain $[0,1]^{3}$. Another objective is to show the convergence of these numerical solutions with the aid of an efficient numerical method.

\subsubsection{Numerical method}

The nonlinear boundary-value problem (5.1) is discretized by a seven-point finite difference formula on a uniform grid with step size $h$. The resulting nonlinear system of equations becomes:

$$
\frac{1}{h^{2}}\left(u_{i_{1}+1, i_{2}, i_{3}}+u_{i_{1}-1, i_{2}, i_{3}}+u_{i_{1}, i_{2}+1, i_{3}}+u_{i_{1}, i_{2}-1, i_{3}}+u_{i_{1}, i_{2}, i_{3}+1}+u_{i_{1}, i_{2}, i_{3}-1}-6 u_{i_{1}, i_{2}, i_{3}}\right)+\lambda \mathrm{e}^{u_{i_{1}, i_{2}, i_{3}}}=0,
$$

where $u_{i_{1}, i_{2}, i_{3}}$ is the approximation at $\left(x_{i_{1}}, x_{i_{2}}, x_{i_{3}}\right), 1 \leq i_{1}, i_{2}, i_{3} \leq J-1$. The Dirichlet boundary conditions read:

$$
u_{0, i_{2}, i_{3}}=u_{J, i_{2}, i_{3}}=u_{i_{1}, 0, i_{3}}=u_{i_{1}, J, i_{3}}=u_{i_{1}, i_{2}, 0}=u_{i_{1}, i_{2}, J}=0 .
$$

We present a multigrid approach for solving the discretized system of nonlinear equations (5.2). This approach is known as the full approximation storage (FAS), where the system of equations (5.2) is solved on each level of the grid throughout the multigrid cycle. For large values of the solution $u$, which could be the case when we look for multiple solutions, diagonal dominance of the tridiagonal matrix is lost. As a result, the tridiagonal matrix may become indefinite on coarser grids. Thus in such cases, standard smoothers such as Gauss-Seidel become unstable. To handle such a difficulty, the MINRES-method (a Krylov subspace method) is introduced as a smoother instead of a Gauss-Seidel method. Next, we describe the numerical strategy to 
compute multiple solutions for $\lambda \in\left(0, \lambda_{c}\right]$.

Recall that the nonlinear discretized system of equations (5.2) on the original fine grid with step size $h=\frac{1}{J}$ on the domain $[0,1]^{3}$ can be written as:

$$
N_{h}\left(u_{h}\right)=0,
$$

where $N_{h}($.$) is the discretized nonlinear operator N(u)=A u+\lambda \mathrm{e}^{u}$. Let $\tilde{u}_{h}$ be the approximate solution on the fine grid $h$ of the nonlinear system (5.2). Restrict the fine grid residual $r_{h}$ to the coarse grid residual $r_{2 h}$ by the restriction operator $R_{h}^{2 h}$. We iterate system (5.2) for $\tilde{u}_{2 h}$ on the coarse grid $2 h$ to approximate the coarse grid error $e_{2 h}$. Interpolate $e_{2 h}$ back to the fine grid error $e_{h}$ by using the interpolating operator $I_{2 h}^{h}$. Make the correction of the fine grid solution as $\tilde{u}_{h}+e_{h}$ (see Algorithm 2 below). This fine-coarse-fine loop is a two-grid V-cycle (we denote it by $V$-cycle) is continued until the tolerance is obtained. Assume that $\nu_{1}$ and $\nu_{2}$ are the pre-smoothing and post-smoothing steps respectively. The complete $F A S$ two grid V-Cycle algorithm (the same as Algorithm 1 in Chapter 4 , repeated for convenience) is explained as:

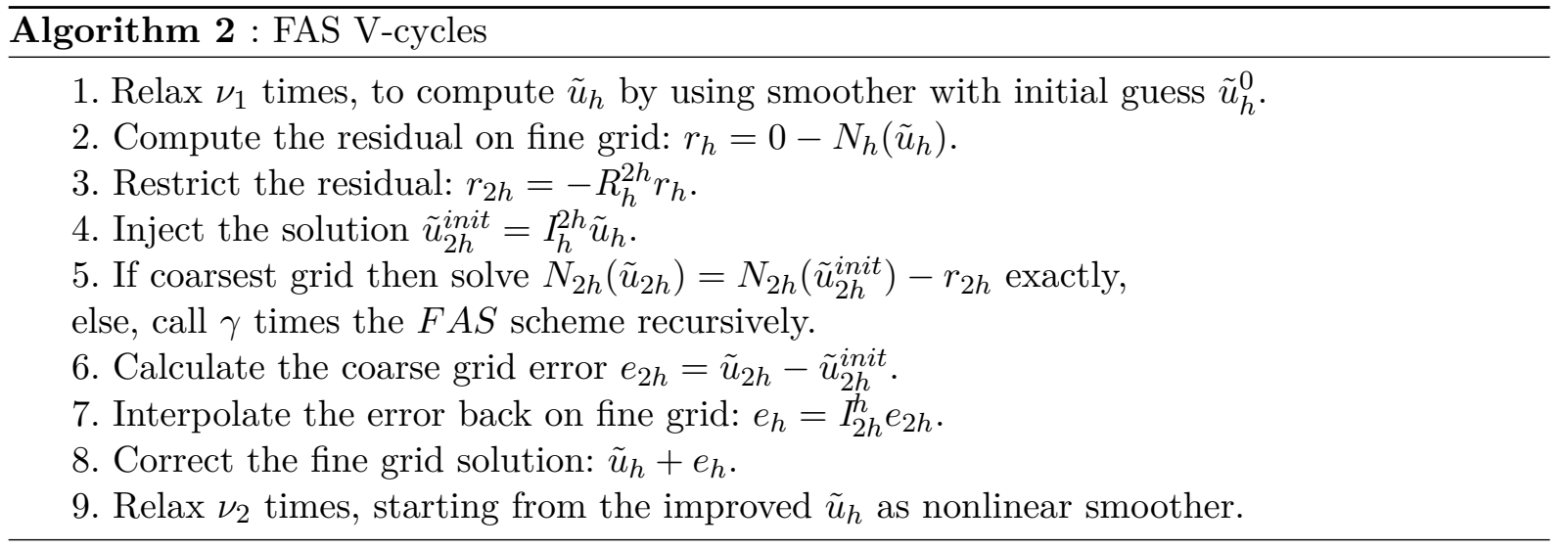

Here, the unknown solution $u$ is relaxed by the MINRES method as a smoother for the discretized linearized equation [138, 97]. The linearized equation for the nonlinear boundaryvalue problem (5.1) becomes:

$$
\Delta u+\lambda f(\tilde{u}) u=\lambda(\tilde{u}-1) f(\tilde{u}),
$$

where $\tilde{u}$ is the current approximate solution of $u$ (mentioned also in step 1 of the Algorithm).

\subsubsection{Numerical results}

In this section, we discuss the numerical implementation of the proposed numerical method for model (5.1) in 3D. We examine the performance of the FAS-MG by using the MINRES method as a smoothing relaxation with an appropriate initial guess. We observed that the proposed multigrid method is very sensitive to the initial guess, particularly for different values of $\lambda$, near to $\lambda_{c}$ and for both turning points: $\lambda_{a}, \lambda_{b}$ for small $h$. The FAS multigrid approach can not perform efficiently, since the coarse grids can not provide an accurate approximation to the solution. In order to make the scaling independent of the grid size $h$, the following residual norm is defined in the numerical procedure:

$$
\|r\|_{2}=\sqrt{\frac{\sum_{i_{1}, i_{2}, i_{3}} r_{i_{1}, i_{2}, i_{3}}^{2}}{J}} .
$$

We choose the initial approximation on the fine grid $h$ as follows:

$$
u_{\text {initial }}=u^{0}=\alpha \sin \left(k \pi x_{i_{1}}\right) \sin \left(k \pi x_{i_{2}}\right) \sin \left(k \pi x_{i_{3}}\right)
$$


with an amplitude $\alpha$ and frequency $k$. These numerical parameters must be specified for each experiment separately. The stopping criteria that we use is: $\|r\|_{2} \leq 10^{-8}$. We choose the multigrid cycle $V(2,2)$ and the coarse grid of size $h=\frac{1}{2}$ with one interior node. We examine the bifurcation behaviour by illustrating the existence of multiple solutions for different values of $\lambda$. Numerical results also provide the convergence for all $\lambda \in\left(0, \lambda_{c}\right]$ on different grids with size $h$. This shows that the proposed numerical strategy is more accurate and efficient than the other numerical techniques in [83, 66] to find the lower and upper solutions of model (5.1) in 3D.

\section{Multiplicity of solutions}

One of the goals of the present numerical study is to compute multiple solutions for the three-dimensional case of GB model 5.1. Note that the numerical solutions depend heavily on the parameter $\lambda$ and grid size $h$. The proposed numerical method worked very well for all $\lambda \in\left(0, \lambda_{c}\right]$ which is one of the main contributions of the present work. Table 5.1 shows the maximum value of the first: $u_{1}$, second: $u_{2}$, third: $u_{3}$ and fourth: $u_{4}$ solutions for different values of $\lambda>0$ with $h=1 / 161$. In Table 5.1, the numerical solution with $*$ indicates that the solution does not converge for the given tolerance on a grid with a high number of grid points $J$.

Table 5.1: The maximum value of the solutions of the three-dimensional GB model (5.1) for $\lambda>0$ and $h=1 / 161$.

\begin{tabular}{|c||c||c||c||c|}
\hline \hline$\lambda$ & $u_{1}$ & $u_{2}$ & $u_{3}$ & $u_{4}$ \\
\hline \hline$\lambda_{c} \approx 9.90257408$ & 1.576134 & - & - & - \\
9.5 & 1.164208 & 2.355216 & - & - \\
8.0 & 0.765826 & 3.357463 & - & - \\
6.5 & 0.515261 & 4.026373 & - & - \\
$\lambda_{a} \approx 6.31432062$ & 0.491024 & 4.188615 & 6.246388 & - \\
6.2 & 0.488463 & 4.065204 & 5.903662 & 6.601146 \\
6.1 & 0.462888 & 4.142452 & 5.802547 & 6.663853 \\
6.0 & 0.467853 & 4.184735 & 5.885483 & 6.724115 \\
5.9 & 0.455032 & 4.213302 & 5.813532 & $6.767654^{*}$ \\
5.5 & 0.405615 & 4.496710 & 5.631087 & $6.973204^{*}$ \\
$\lambda_{b} \approx 4.8261277$ & 0.326448 & 5.163705 & $7.1683172^{*}$ & - \\
4.8 & 0.321485 & - & $7.198551^{*}$ & - \\
4.5 & 0.311926 & $7.244282^{*}$ & - & - \\
2.0 & 0.124607 & $7.600145^{*}$ & - & - \\
1.5 & 0.089335 & $7.812563^{*}$ & - & - \\
1.0 & 0.056903 & $8.162511^{*}$ & - & - \\
0.5 & 0.028631 & - & - & - \\
0.3 & 0.008993 & - & - & - \\
0.1 & 0.007436 & - & - & - \\
\hline
\end{tabular}

\section{Numerical convergence in the case of multiple solutions}

The convergence of the numerical solutions, especially for the solutions $u_{2}, u_{3}, u_{4}$, is of great importance as it confirms the existence of more than two solutions. It is the first time, as far as we know, that the numerical convergence of these multiple solutions on small grid sizes is described. With the help of FAS-MG, we successfully obtain numerical convergence for both small values of $\lambda$ and the new bifurcation points $\lambda_{a}, \lambda_{b}$ as well as for the critical value $\lambda_{c}$. All 
these aspects of the numerical convergence for different values of $\lambda$ for a large number of grid points $J$ are discussed in detail.

For the small parameter value: $\lambda=0.1$, Figure 5.1 shows the numerical convergence of the solution on different grid sizes. It shows the effectiveness of the FAS-MG method for the GB model in 3D. For $\lambda \in\left(0, \lambda_{b}\right)$, we are able to compute two solutions, in which the first solution

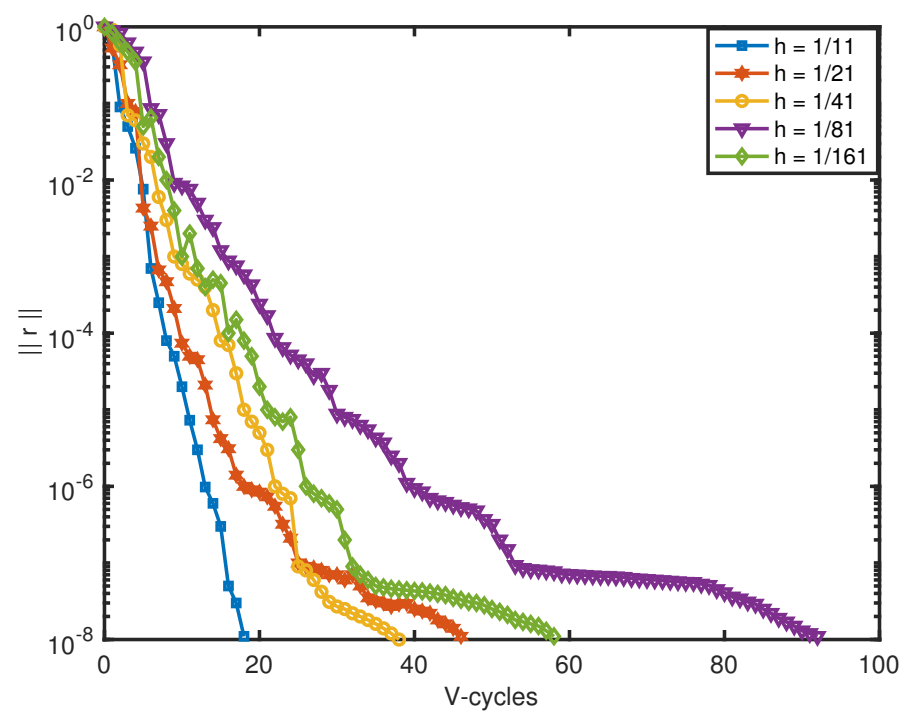

Figure 5.1: Numerical convergence for a single solution of the three-dimensional GB model (5.1) for $\lambda=0.1$ on decreasing grid sizes $h$.

has converged for small values of $h$, whereas the second solution is a spurious solution (see also Table 5.1). To explain this convergence behaviour we take $\lambda=1$ as a characteristic example. The numerical convergence of both solutions for $\lambda=1$ is given in Table 5.2. Figure 5.2 demonstrates

Table 5.2: The number of $\mathrm{V}$-cycles and the CPU time for first solution of the three-dimensional GB model (5.1) for $\lambda=1$ and decreasing values of grid sizes $h$.

\begin{tabular}{|c||c||c||c|c|}
\hline \hline \multicolumn{1}{|c||}{ Grid size } & \multicolumn{2}{c||}{ First solution } & \multicolumn{2}{c|}{ Second solution } \\
\hline$h$ & V-cycles & Time (seconds) & V-cycles & Time (seconds) \\
\hline \hline $1 / 11$ & 8 & 66.2208 & 48 & 796.1986 \\
$1 / 21$ & 12 & 186.2955 & $100^{*}$ & 1044.4375 \\
$1 / 41$ & 12 & 432.4789 & $100^{*}$ & 1331.5509 \\
$1 / 81$ & 18 & 805.0368 & $100^{*}$ & 1905.0087 \\
$1 / 161$ & 16 & 1416.9937 & $100^{*}$ & 2643.6743 \\
\hline
\end{tabular}

* indicates that no numerical convergence was reached.

that the proposed method successfully converges to the unique solution for the critical value of $\lambda_{c}$ on fine grids. For both new bifurcation turning points $\lambda_{a} \approx 6.31432062$ and $\lambda_{b} \approx 4.82612776$, three solutions are obtained (for $\lambda_{b}$ there is one spurious solution). For $\lambda_{a}$ all solutions $u_{1}, u_{2}, u_{3}$ have numerically converged on a large number of grid points $J$ as shown in Figure 5.3. However, for $\lambda_{b}$, one of the solutions, viz., $u_{3}$ did not converge on a fine grid (see Figure 5.4).

For $\lambda \in\left(\lambda_{b}, \lambda_{a}\right)$, we are able to compute four numerical solutions. In particular, the computation of the fourth solution, is a difficult task, as MG-methods are very sensitive to the choice of the initial guess. However, the FAS-MG method efficiently resolved the computational 


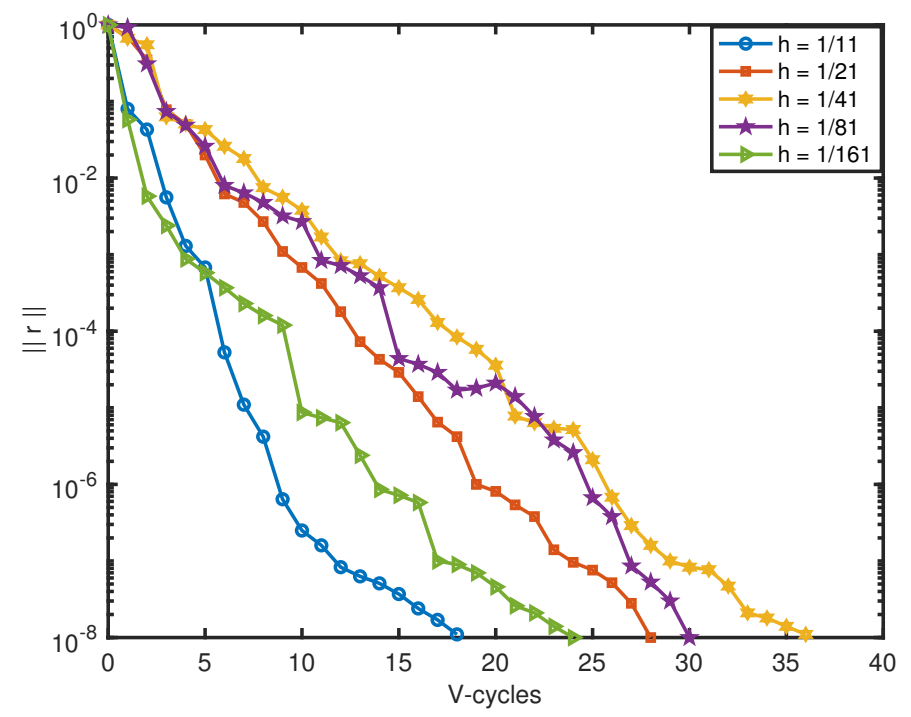

Figure 5.2: Numerical convergence for the unique solution of the three-dimensional GB model (5.1) for the critical value $\lambda_{c}$ and decreasing values of $h$.

difficulties with the MINRES method as a smoother and with an appropriate initial guess. It is important to mention that the proposed MG-method successfully achieved convergence for all $\lambda \in\left(0, \lambda_{c}\right]$. To demonstrate the existence of the three solutions, we present the numerical convergence of such solutions $u_{1}, u_{2}$ and $u_{3}$ for $\lambda=5.5$. This is shown in Tables 5.3, 5.4. Figure 5.5 shows the isosurface plots of the three solutions for $\lambda=5.5$ on a grid with $41^{3}$ grid points.

Table 5.3: The number of V-cycles and the CPU time for the first and second solutions of the three-dimensional GB model (5.1) for $\lambda=5.5$ and decreasing values of $h$.

\begin{tabular}{|c||c||c||c||c|}
\hline \hline \multicolumn{1}{|c||}{ Grid size } & \multicolumn{2}{c||}{ First-solution } & \multicolumn{2}{c|}{ Second-solution } \\
\hline$h$ & V-cycles & Time (seconds) & V-cycles & Time (seconds) \\
\hline \hline $1 / 11$ & 8 & 41.8657 & 28 & 158.9705 \\
$1 / 21$ & 12 & 118.0548 & 22 & 293.3296 \\
$1 / 41$ & 8 & 288.6603 & 48 & 576.2328 \\
$1 / 81$ & 12 & 443.5499 & 36 & 825.5191 \\
$1 / 161$ & 18 & 757.4487 & 42 & 1196.1645 \\
\hline
\end{tabular}

To illustrate the existence of a fourth solution, we take $\lambda=6$ as a characteristic example. Figure 5.6 presents the isosurface plots of the four solutions: $u_{1}, u_{2}, u_{3}, u_{4}$ for $\lambda=6$ on a grid with $41^{3}$ grid points. Numerical convergence is confirmed in Figure 5.7.

\section{A bifurcation curve in three space dimensions}

Our numerical investigations for $\lambda \in\left(0, \lambda_{c}\right]$ give rise to a qualitative picture of the bifurcation curve in three space dimensions for model (5.1), which is quite different from the one and twodimensional case. For the three-dimensional case, we present the bifurcation curve in Figure 5.8, where two new turning points $\lambda_{a} \approx 6.31432062$ and $\lambda_{b} \approx 4.8261277$ are identified. Different numerical experiments for the values of $\lambda$ lead to the numerical value $\lambda_{c} \approx 9.90257408$. Figure 5.8 incorporates the four solutions, two new turning points and one critical point for $\lambda \in\left(0, \lambda_{c}\right]$. 

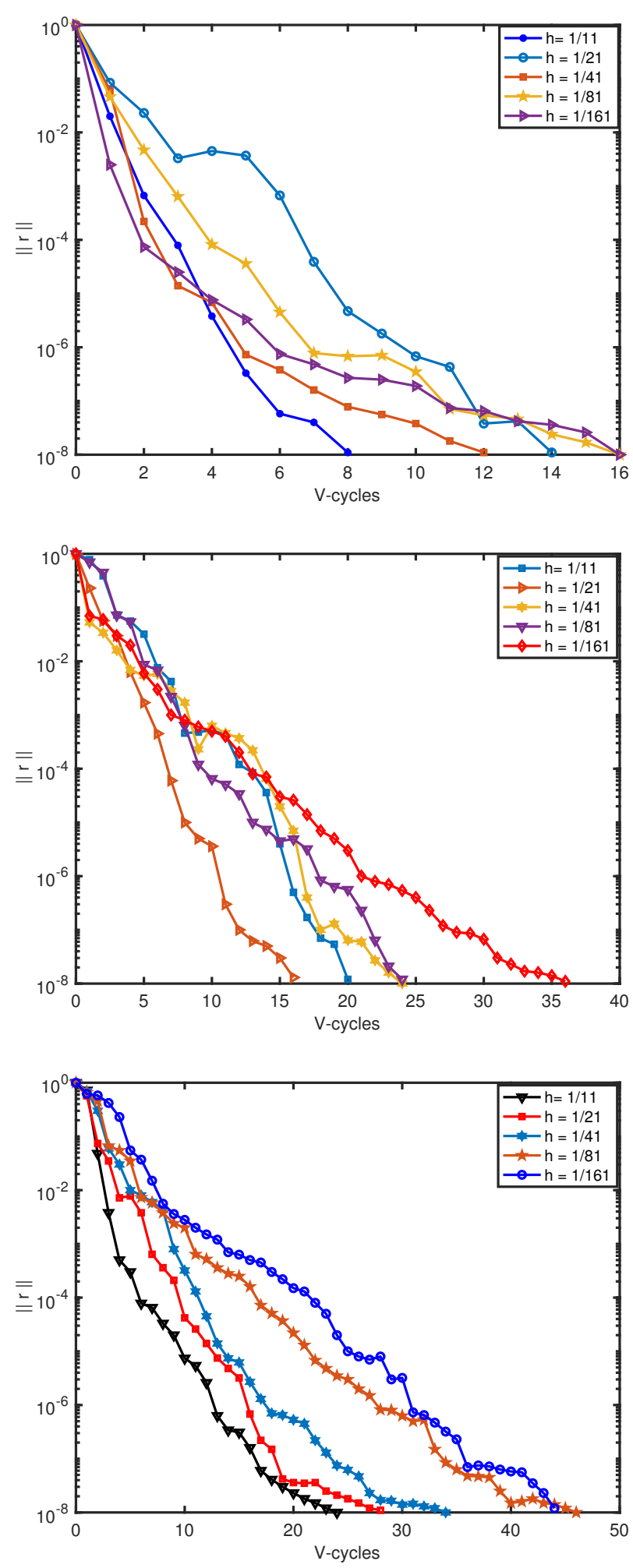

Figure 5.3: Numerical convergence of the three solutions: first solution: $u_{1}$ (top), second solution: $u_{2}$ (middle) and third solution: $u_{3}$ (bottom), respectively, for $\lambda_{a} \approx 6.31432062$ on decreasing values of grid sizes $h$ of the three-dimensional GB model. 


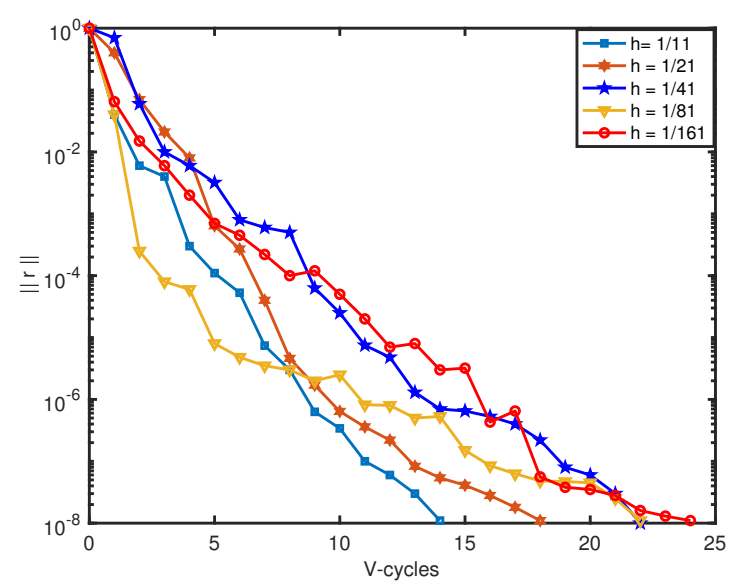

first solution: $u_{1}$

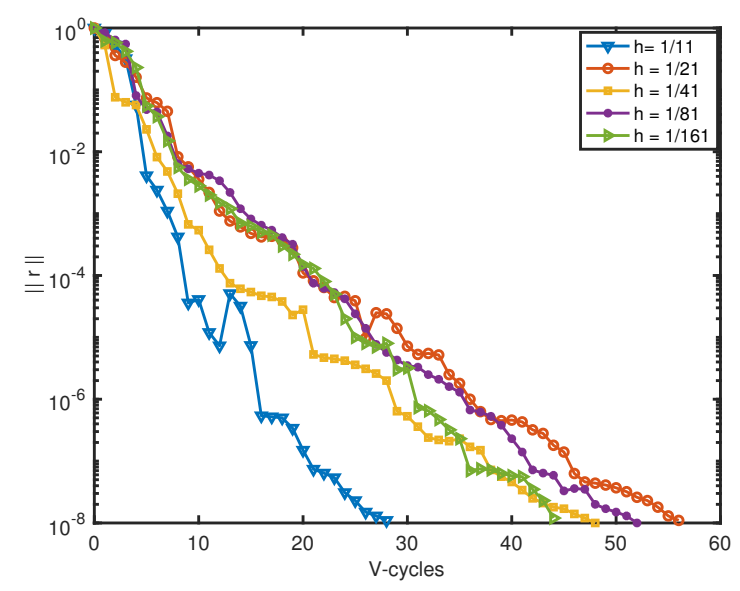

second solution: $u_{2}$

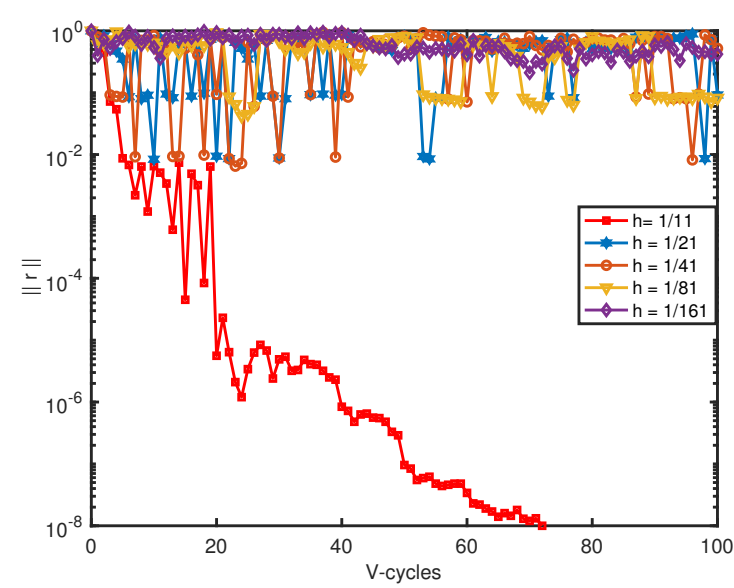

third spurious solution*: $u_{3}^{*}$

Figure 5.4: Numerical convergence of two solutions: first solution: $u_{1}$ (top), second solution: $u_{2}$ (middle) and divergence of the third spurious solution : $u_{3}^{*}$ (bottom), respectively, for $\lambda_{b} \approx$ 4.82612776 on decreasing values of grid sizes $h$ of the three-dimensional GB model. 

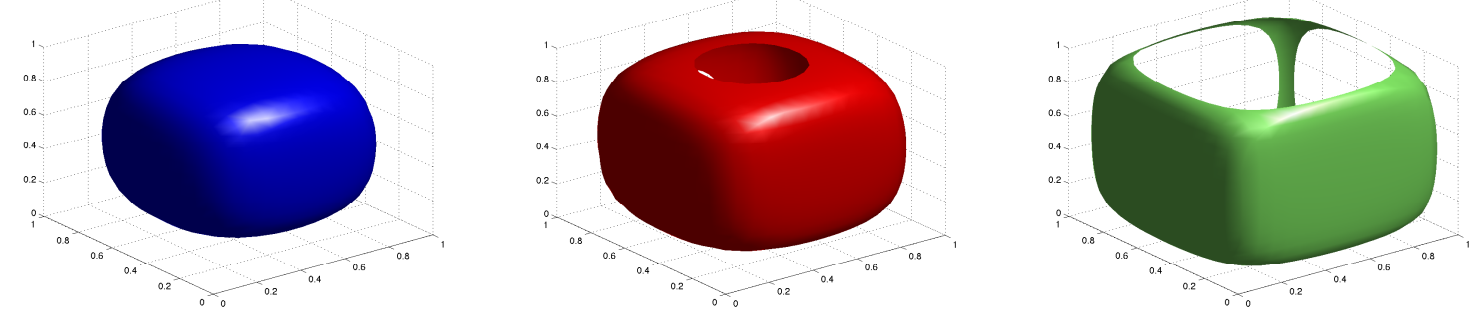

Figure 5.5: Iso-surface plots of three solutions: $u_{1}, u_{2}$ and $u_{3}$, respectively, of model (5.1) in three space dimensions for $\lambda=5.5$ at the iso-value $=0.35$.

Table 5.4: The number of V-cycles and the CPU time for the third and fourth spurious solution of the three-dimensional GB model (5.1) for $\lambda=5.5$ and decreasing values of $h$.

\begin{tabular}{|c||c||c||c||c|}
\hline \hline \multicolumn{1}{|c||}{ Grid size } & \multicolumn{2}{c||}{ Third-solution } & \multicolumn{2}{c|}{ Fourth-solution } \\
\hline$h$ & V-cycles & Time (seconds) & V-cycles & Time (seconds) \\
\hline \hline $1 / 11$ & 36 & 242.3997 & 72 & 707.5586 \\
$1 / 21$ & 46 & 426.2311 & $100^{*}$ & 1494.4805 \\
$1 / 41$ & 42 & 974.5904 & $100^{*}$ & 1857.3858 \\
$1 / 81$ & 52 & 1506.6630 & $100^{*}$ & 2643.1886 \\
$1 / 161$ & 86 & 2133.0085 & $100^{*}$ & 3326.8374 \\
\hline
\end{tabular}

* indicates that no numerical convergence was reached.

In three dimensions, it can be seen clearly that for $\lambda \geq 6$ we found four solutions (denoted by " $4^{\text {th }}$ - solution solid line") and for $\lambda \leq 5.9$, one solution out of the four solutions is a spurious one (denoted by " $4^{\text {th }}-$ solution* dashed-line") within $\left(\lambda_{b}, \lambda_{a}\right)$.

\subsection{The n-dimensional radial case}

Motivated by the three-dimensional numerical results in Cartesian coordinates, we will now investigate the multiplicity of solutions and the bifurcation behaviour in $n$ space dimensions. In this section, we look for multiple solutions of nonlinear BVP (5.1) on a special domain $\Omega$, viz. the $n$-dimensional ball $B:=\left\{\overrightarrow{\mathrm{x}} \in \mathbb{R}^{n}:\|\overrightarrow{\mathrm{x}}\|_{2}<1\right\}$. For this, we first transform model (5.1) using $n$-dimensional spherical coordinates. All solutions of (5.1) on $B$ can be proved to be positive, because of the maximum principle and the radial symmetry (for more details, see [11]). We transform the equation from Cartesian coordinates to $n$-dimensional spherical coordinates. After that we compute numerical solutions of the $n$-dimensional nonlinear problem in spherical coordinates.

\subsubsection{Spherical coordinates in $n$ space dimensions}

In this section, we transform the equation in $n$ space dimensions from Cartesian coordinates $\left(x_{1}, x_{2}, x_{3}, \ldots, x_{n}\right)$ to (hyper-)spherical coordinates $\left(\rho, \phi_{1}, \phi_{2}, \phi_{3}, \ldots, \phi_{n-1}\right)$ and investigate radially symmetric solutions of nonlinear BVP (5.1). In Cartesian coordinates, which are mainly useful for rectangular domains, the Laplace operator has the form:

$$
\Delta=\sum_{k=1}^{n} \frac{\partial^{2}}{\partial x_{k}^{2}} .
$$




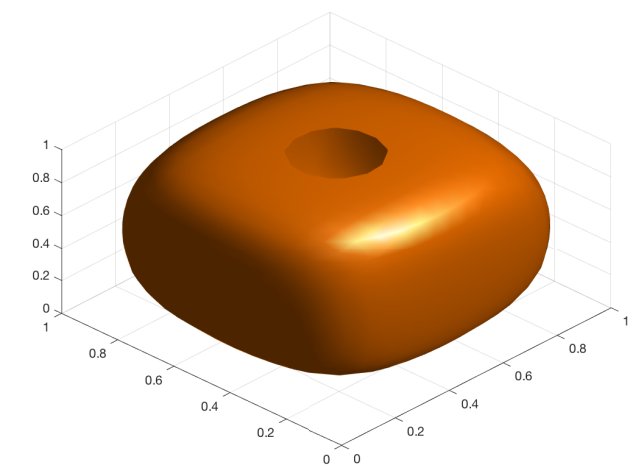

first solution: $u_{1}$

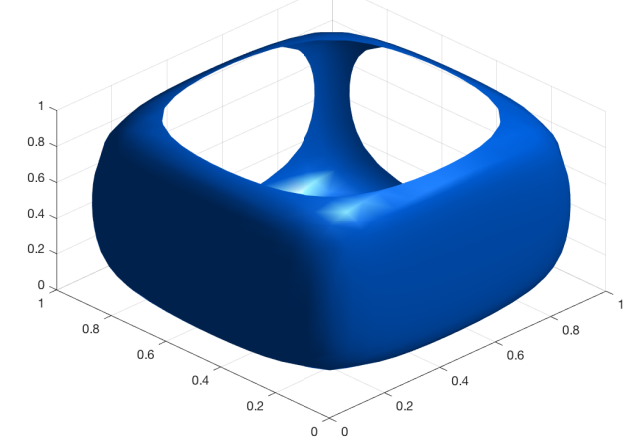

third solution: $u_{3}$

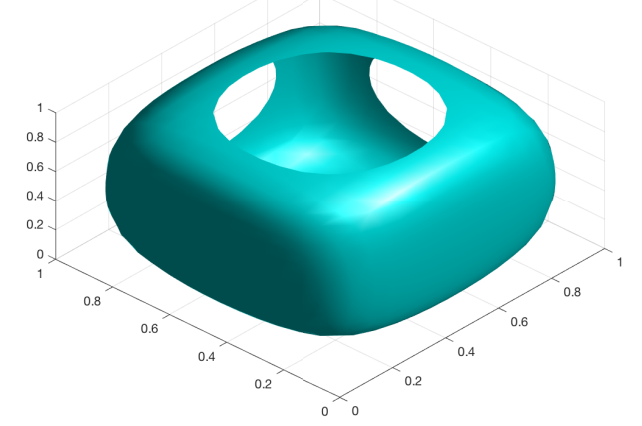

second solution: $u_{2}$

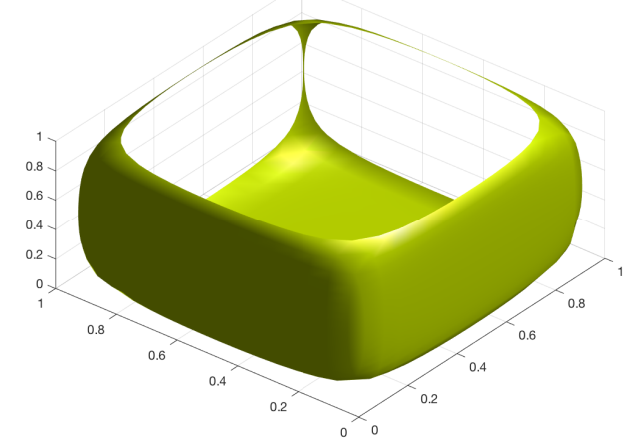

fourth solution: $u_{4}$

Figure 5.6: Iso-surface plots of four solutions of the three-dimensional GB model (5.1) for $\lambda=6.0$ at the iso-value $=0.42$. 


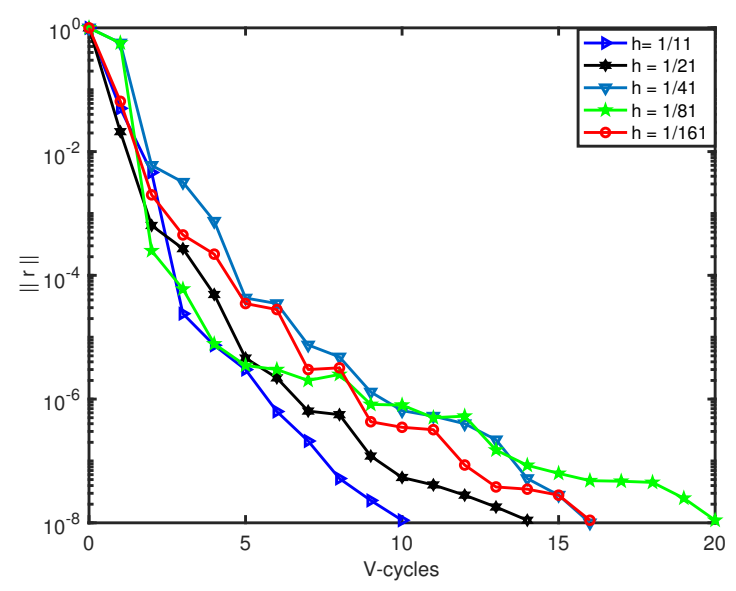

convergence of first solution

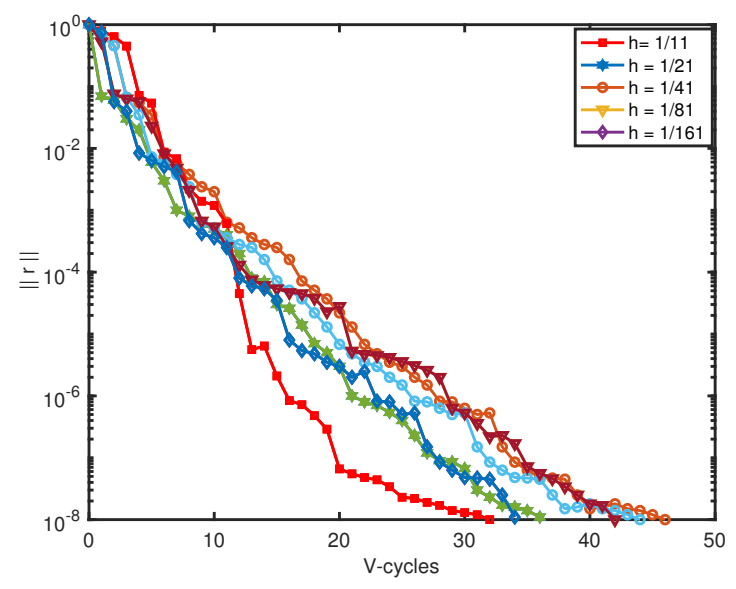

convergence of third solution

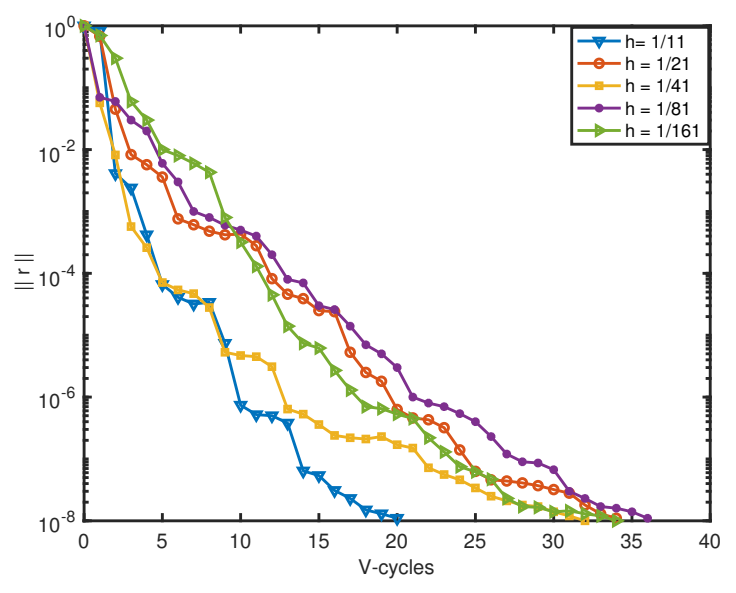

convergence of second solution

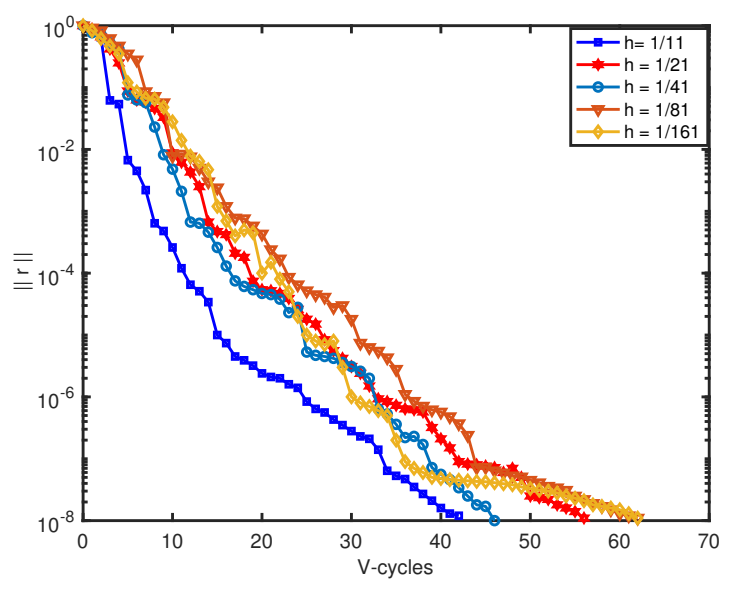

convergence of fourth solution

Figure 5.7: Numerical convergence for the four solutions: $u_{1}, u_{2}, u_{3}$ and $u_{4}$, respectively, as a function of the number of V-cycles and grid size $h$ for $\lambda=6.0$ of the three-dimensional GB model (5.1). 


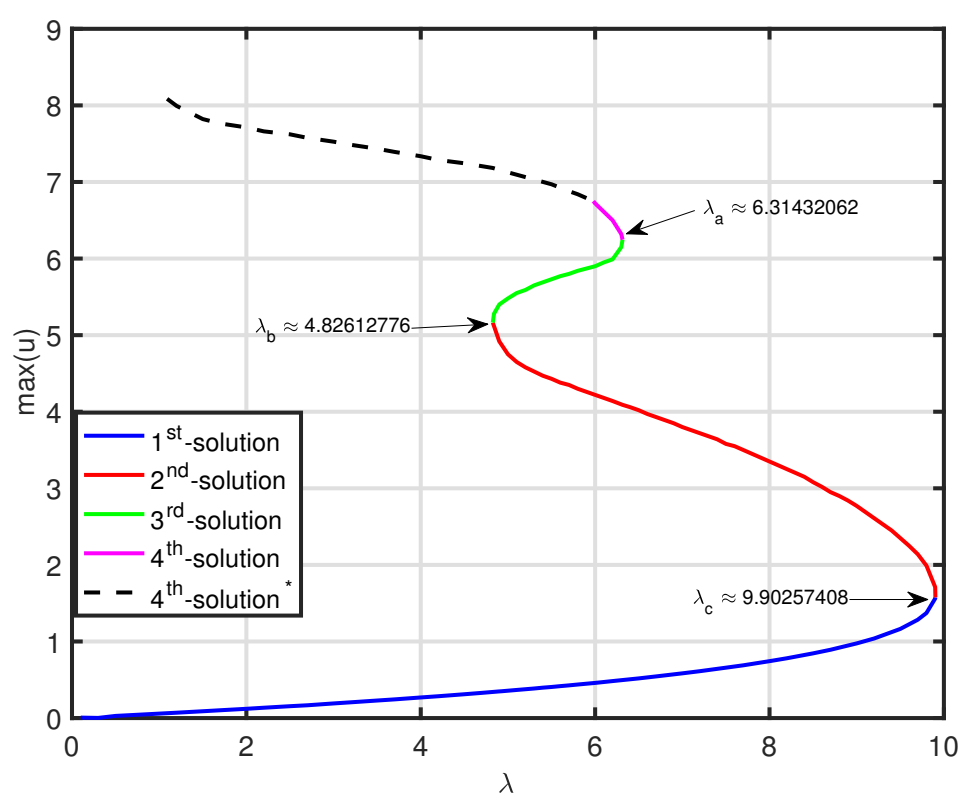

Figure 5.8: Bifurcation curve for GB model (5.1) in three space dimensions. Solid lines indicate the four numerically converged solutions (see Figure 5.7) and the dashed line shows spurious numerical solutions. The two new turning points $\lambda_{a} \approx 6.31432062$ and $\lambda_{b} \approx 4.8261277$. The third critical value $\lambda_{c} \approx 9.90257408$.

For more complicated domains, it is convenient to work with other coordinate systems. A general, so-called curvilinear, coordinate system can be written as follows:

$$
\left\{\begin{array}{c}
x_{1}=x_{1}\left(q_{1}, q_{2}, q_{3}, \ldots, q_{n}\right) \\
x_{2}=x_{2}\left(q_{1}, q_{2}, q_{3}, \ldots, q_{n}\right) \\
x_{3}=x_{3}\left(q_{1}, q_{2}, q_{3}, \ldots, q_{n}\right) \\
\vdots \\
x_{n}=x_{n}\left(q_{1}, q_{2}, q_{3}, \ldots, q_{n}\right)
\end{array}\right.
$$

where $\left(q_{1}, q_{2}, q_{3}, \ldots, q_{n}\right)$ are orthogonal curvilinear coordinates and $\left(x_{1}, x_{2}, x_{3}, \ldots, x_{n}\right)$ Cartesian coordinates, respectively. By considering equation (5.7), we can write the tangent vectors to the curvilinear coordinates in terms of scaling coefficients and unit vectors as:

$$
\sum_{k=1}^{n} \frac{\partial x_{k}}{\partial q_{j}}=\zeta_{j} \mathbf{e}_{j}, \quad j=1,2, \ldots, n,
$$

where $\mathbf{e}_{j}$ are the unit vectors in the direction of the curvilinear coordinates $q_{j}$, respectively. The scaling coefficient $\zeta$ is defined as:

$$
\zeta=\prod_{j=1}^{n} \zeta_{j}
$$

where

$$
\zeta_{j}=\left[\sum_{k=1}^{n}\left(\frac{\partial x_{k}}{\partial q_{j}}\right)^{2}\right]^{1 / 2}, \quad j=1,2,3, \ldots, n
$$


Then, a general expression for the Laplacian $\Delta$ in orthogonal curvilinear coordinates is of the form (see also [63]):

$$
\Delta=\frac{1}{\zeta} \sum_{k=1}^{n} \frac{\partial}{\partial q_{k}}\left(\frac{\zeta}{\zeta_{k}^{2}} \frac{\partial}{\partial q_{k}}\right)
$$

For the special choice of spherical coordinates, we find:

$$
\left\{\begin{array}{l}
x_{1}=\rho \cos \phi_{1}, \\
x_{2}=\rho \sin \phi_{1} \cos \phi_{2}, \\
x_{3}=\rho \sin \phi_{1} \sin \phi_{2} \cos \phi_{3}, \\
x_{4}=\rho \sin \phi_{1} \sin \phi_{2} \sin \phi_{3} \cos \phi_{4} \\
\vdots \\
x_{n-1}=\rho \prod_{b=1}^{n-2} \sin \phi_{b} \cos \phi_{n-1}, \\
x_{n}=\rho \prod_{b=1}^{n-1} \sin \phi_{b} .
\end{array}\right.
$$

where $\rho>0, \quad 0 \leq \phi_{b} \leq \pi(b=1,2,3, \ldots, n-2)$ and $0 \leq \phi_{n-1} \leq 2 \pi$. The scaling coefficients in equation (5.8) are explicitly written as

$$
\left\{\begin{array}{l}
\zeta_{1}=1, \\
\zeta_{2}=\rho, \\
\zeta_{3}=\rho \sin \phi_{1}, \\
\vdots \\
\zeta_{n}=\rho \prod_{b=1}^{n-2} \sin \phi_{b} .
\end{array}\right.
$$

The Laplace operator (5.6) in $n$-dimensional spherical coordinates, making use of (5.8), (5.9) and (5.10), becomes

$$
\begin{aligned}
\Delta & =\frac{1}{\rho^{n-1}} \frac{\partial}{\partial \rho}\left(\rho^{n-1} \frac{\partial}{\partial \rho}\right) \\
& +\frac{1}{\rho^{2}} \sum_{b=1}^{n-2}\left[\prod_{a=b+1}^{n-1} \frac{1}{\sin ^{2} \phi_{a}}\left(\frac{1}{\sin ^{b-1} \phi_{b}}\left(\frac{\partial}{\partial \phi_{b}} \sin ^{b-1} \phi_{b} \frac{\partial}{\partial \phi_{b}}\right)\right)\right] \\
& +\frac{1}{\rho^{2}}\left(\frac{1}{\sin ^{n-2} \phi_{n-1}} \frac{\partial}{\partial \phi_{n-1}}\left(\sin ^{n-2} \phi_{n-1} \frac{\partial}{\partial \phi_{n-1}}\right)\right) .
\end{aligned}
$$

For two dimensions (5.11) becomes

$$
\Delta=\frac{1}{\rho} \frac{\partial}{\partial \rho}\left(\rho \frac{\partial}{\partial \rho}\right)+\frac{1}{\rho^{2}} \frac{\partial^{2}}{\partial \phi_{1}^{2}}
$$

and for three dimensions

$$
\Delta=\frac{1}{\rho^{2}} \frac{\partial}{\partial \rho}\left(\rho^{2} \frac{\partial}{\partial \rho}\right)+\frac{1}{\rho^{2}} \frac{1}{\sin ^{2} \phi_{2}} \frac{\partial^{2}}{\partial \phi_{1}^{2}}+\frac{1}{\rho^{2} \sin \phi_{2}} \frac{\partial}{\partial \phi_{2}}\left(\sin \phi_{2} \frac{\partial}{\partial \phi_{2}}\right) .
$$

In the following section, we transform the nonlinear PDE (5.1) defined on the ball $B$, making use of the $n$-dimensional spherical coordinates. 


\subsubsection{A boundary-value problem in $n$-dimensional spherical coordinates}

We rewrite the nonlinear problem (5.1) in $n$-dimensional spherical coordinates (5.9) as:

$$
\begin{array}{r}
\frac{1}{\rho^{n-1}} \frac{\partial}{\partial \rho}\left(\rho^{n-1} \frac{\partial u}{\partial \rho}\right)+\frac{1}{\rho^{2}} \sum_{b=1}^{n-2}\left[\prod_{a=b+1}^{n-1} \frac{1}{\sin ^{2} \phi_{a}}\left(\frac{1}{\sin ^{b-1} \phi_{b}}\left(\frac{\partial}{\partial \phi_{b}} \sin ^{b-1} \phi_{b} \frac{\partial u}{\partial \phi_{b}}\right)\right)\right] \\
+\frac{1}{\rho^{2}}\left(\frac{1}{\sin ^{n-2} \phi_{n-1}} \frac{\partial}{\partial \phi_{n-1}}\left(\sin ^{n-2} \phi_{n-1} \frac{\partial u}{\partial \phi_{n-1}}\right)\right)+\lambda \mathrm{e}^{u}=0 .
\end{array}
$$

Next, we simplify partial differential equation (5.12) using symmetry properties. If $\Omega$ is a ball $B$ in $\mathbb{R}^{n}$ centered at 0 , then we could seek for radially symmetric solutions. More precisely, for $\Omega=B=\left\{\overrightarrow{\mathrm{x}} \in \mathbb{R}^{n}:\|\overrightarrow{\mathrm{x}}\|_{2}<1\right\}$, let $u \in C^{2}(\Omega, \mathbb{R})$ be a positive solution of (5.12), then $u$ is radially symmetric (details can be found in [11]). This implies that the derivatives w.r.t. $\phi_{1}, \phi_{2}, \ldots, \phi_{n}$ are identically zero and equation (5.12) reduces to the ODE:

$$
\frac{1}{\rho^{n-1}} \frac{\partial}{\partial \rho}\left(\rho^{n-1} \frac{\partial u}{\partial \rho}\right)+\lambda \mathrm{e}^{u}=0
$$

For $\rho:=\|\overrightarrow{\mathrm{x}}\|_{2}$, we have $u=u(\rho) \in C^{2}[0,1]$ and $\frac{\partial u}{\partial \rho}(\rho)=u^{\prime}(\rho)<0$ for $\rho \in(0,1)$ (details are given in [11]). This implies that any positive solution of BVP (5.1) is a solution of the following ODE:

$$
u^{\prime \prime}+\frac{n-1}{\rho} u^{\prime}+\lambda \mathrm{e}^{u}=0, \quad 0<\rho<1
$$

with boundary conditions $u^{\prime}(0)=0$ and $u(1)=0$. In the next section, we discuss the multiplicity results and the bifurcation behaviour of nonlinear ODE (5.14) for different values of the dimension parameter $n$.

\subsubsection{Theoretical results for $n$ space dimensions in spherical coordinates}

For ODE (5.14), theoretical results are given in [11] based on the dimension parameter $n$ in the following way:

1. For $n=1$, there exists a $\lambda_{c}>0$ such that

(a) for each $\lambda \in\left(0, \lambda_{c}\right)$, there are two solutions.

(b) for $\lambda=\lambda_{c}$, there is a unique solution.

(c) for $\lambda>\lambda_{c}$, there are no solutions.

2. For $n=2$, define $\lambda_{c}=2$. Then

(a) for each $\lambda \in\left(0, \lambda_{c}\right)$, there are two solutions.

(b) for $\lambda=\lambda_{c}$, there is a unique solution.

(c) for $\lambda>\lambda_{c}$, there are no solutions.

3. For $3 \leq n \leq 9$, define $\tilde{\lambda}=2(n-2)$. Then there exists a $\lambda_{c}>\tilde{\lambda}$ such that

(a) for $\lambda=\lambda_{c}$, there is a unique solution.

(b) for $\lambda>\lambda_{c}$, there are no solutions. 
(c) for $\lambda=\tilde{\lambda}$, there is a countable infinite of solutions.

(d) for each $\lambda \in\left(0, \lambda_{c}\right) \backslash\{\tilde{\lambda}\}$, there is a finite number of solutions.

4. For $n \geq 10$, define $\lambda_{c}=2(n-2)$. Then

(a) for $\lambda \geq \lambda_{c}$, there are no solutions.

(b) for $\lambda \in\left(0, \lambda_{c}\right)$, there is a unique solution.

We are going to numerically detect these solutions in the next section.

\subsubsection{Numerical experiments}

We compute the numerical solutions of the nonlinear ODE (5.14) by using a simple shooting method. For the numerical time-integration as a part of the shooting method, we use the MATLAB function ode 45 with a tolerance $10^{-10}$. Several experiments are performed for different values of $n$. We present the bifurcation behaviour as a function of the parameter $\lambda$ and the spatial dimension parameter $n$. The bifurcation curves show the relation between $u(0)$ (maximum value of a solution) and $u(1)$. The numerical experiments confirm all theoretical results as mentioned in section 5.5.3.

\section{Experiment 1}

We present numerical solutions of ODE (5.14) for $n=1$ and different values of $\lambda$. Figure 5.9 (left), illustrates the two numerical solutions $u_{1}$ and $u_{2}$ for $\lambda=0.8$ and the unique solution $u_{c}$ for $\lambda_{c}$. The critical value $\lambda_{c} \approx 0.86752074$ is calculated numerically. A bifurcation diagram for $n=1$ of nonlinear ODE (5.14) for different values of $\lambda$ is provided, see Figure 5.9 (right), wherein the solutions can be identified as a zero of the curve $(u(0), u(1))$. These bifurcation curves show that for $0<\lambda<\lambda_{c}$ there exist exactly two solutions, precisely one solution for $\lambda=\lambda_{c}$ and no solution exists for $\lambda>0$.
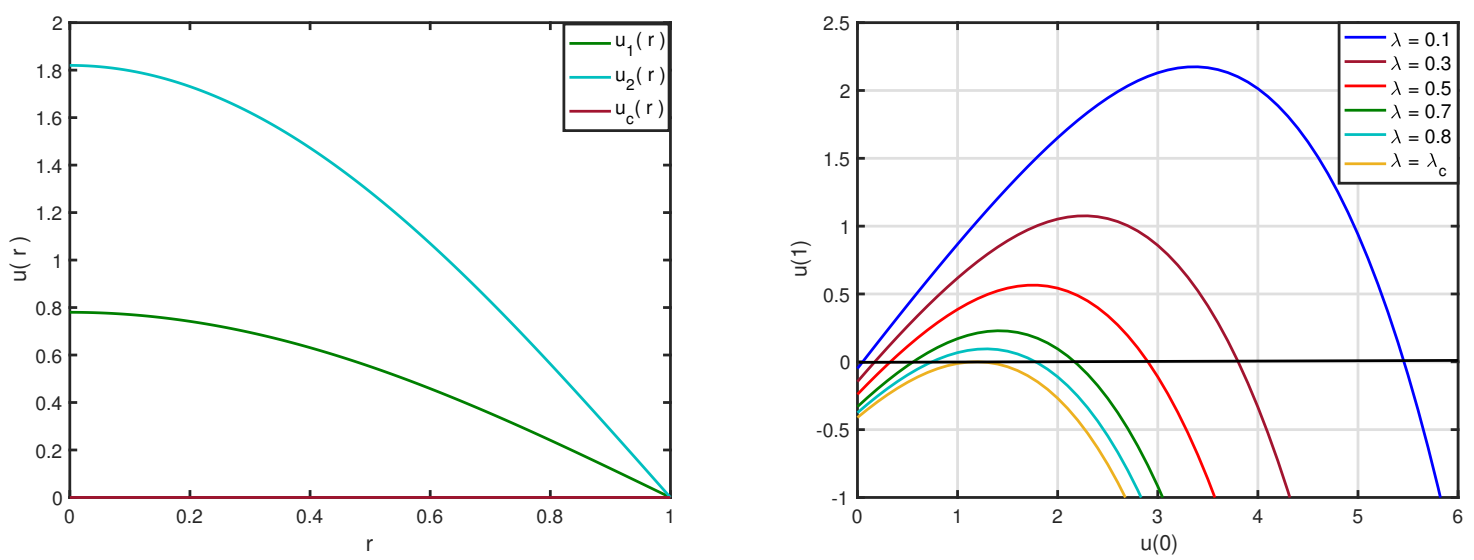

Figure 5.9: For the dimension parameter $n=1$ of nonlinear ODE (5.14): two numerical solutions $u_{1}(r), u_{2}(r)$ for $\lambda=0.8$ and one solution $u_{c}(r)$ for the critical value $\lambda_{c}$ (left) and bifurcation curves for different values of $\lambda$ (right). 


\section{Experiment 2}

The numerical results of ODE (5.14) for $n=2$ are now discussed. Two numerical solutions exist for $\lambda \in\left(0, \lambda_{c}\right)$ and one solution $u_{c}$ for $\lambda_{c}$. These aspects are presented in Figure 5.10. For this case, the critical value is $\lambda_{c} \approx 2$. We show the bifurcation behaviour in $n$ space dimensions for

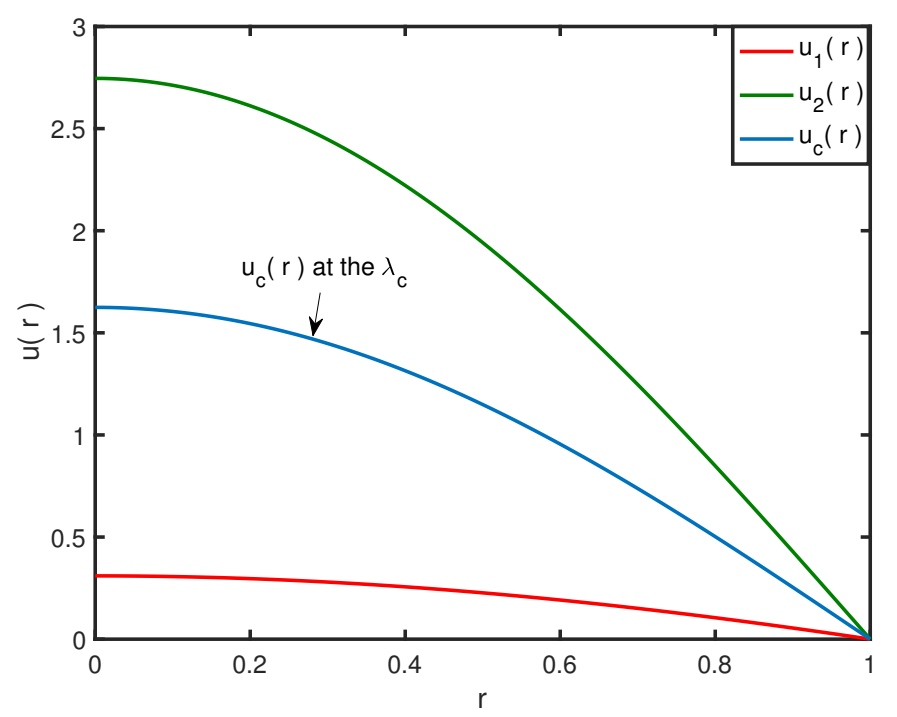

Figure 5.10: Numerical solutions of nonlinear model (5.14) for $n=2$ : two solutions $u_{1}(r), u_{2}(r)$ for $\lambda=1$ and one solution $u_{c}(r)$ for the critical value $\lambda_{c}=2$.

ODE (5.14) with $n=2$ and different values of $\lambda$. The curves in Figure 5.11 show the maximum value of the two solutions: $u_{1}, u_{2}$ for $\lambda \in\left(0, \lambda_{c}\right)$, a unique solution for $\lambda_{c}$ and no solution for $\lambda>\lambda_{c}$. This behaviour is explained theoretically in section 5.5.3.
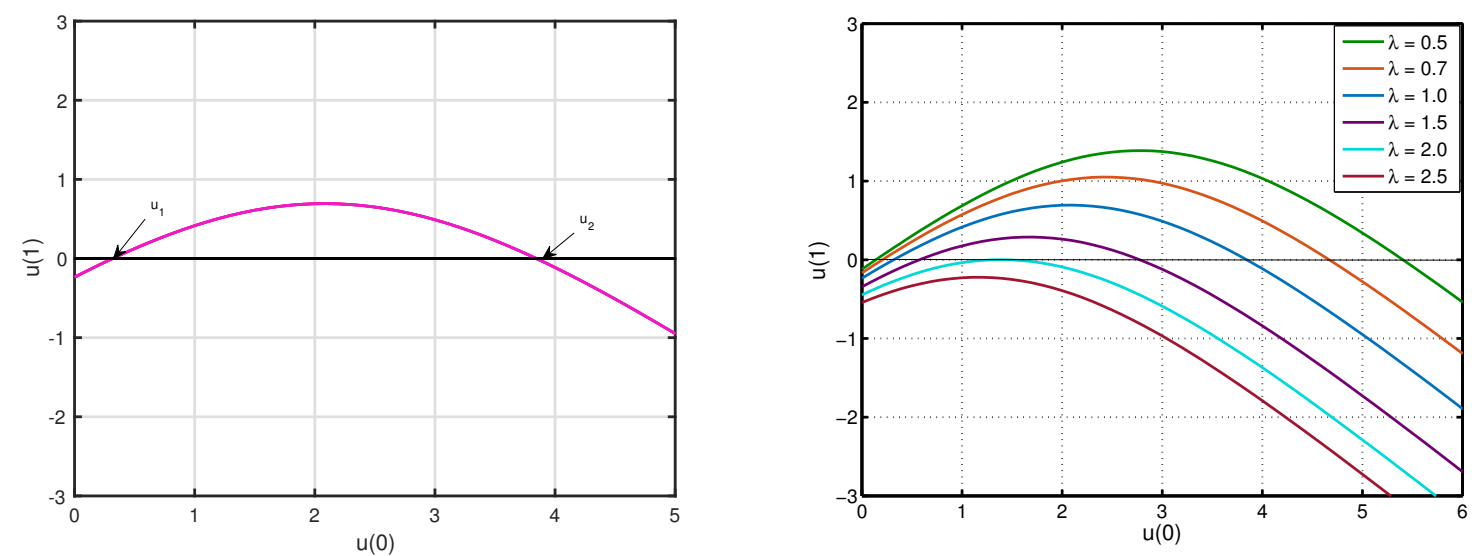

Figure 5.11: Bifurcation curves for $n=2$ of model (5.14): two numerical solutions $u_{1}, u_{2}$ are identified as a zero of the curve $(u(0), u(1))$ for $\lambda=1$ (left) and for the other values of $\lambda$ (right). 


\section{Experiment 3}

We provide numerical results of BVP (5.14) for $3 \leq n \leq 9$. The theoretical results for this case are quite different from the cases $n=1$ and $n=2$. We will show the existence of multiple solutions for different values of $\lambda$. As a characteristic example we take $n=3$. For different values of $\lambda$, one, two, three or more solutions are depicted in Figure 5.12. For $n=3$, we have $\tilde{\lambda}=2$. We compute several solutions for $\lambda \in\left(0, \lambda_{c}\right) \backslash\{\tilde{\lambda}\}$. For this, we take $\lambda=1.999$ and computed the seven solutions, see in Figure 5.12 (right). For this characteristic example, a unique solution $u_{c}$ exists for critical value $\lambda_{c}$. The critical value for $n=3$ is numerically found to be $\lambda_{c} \approx 3.352731$. The theoretical results for $3 \leq n \leq$ as described in section 5.5.3, are confirmed numerically in
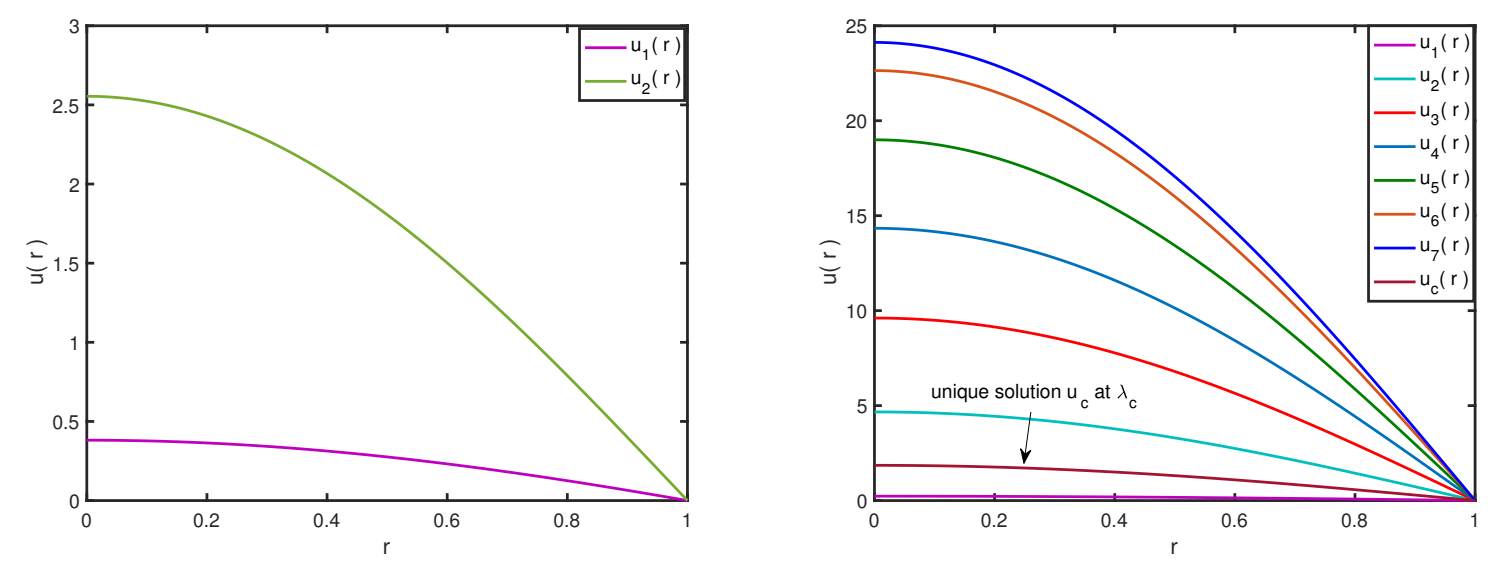

Figure 5.12: Numerical solutions of nonlinear ODE (5.14) for $n=3$. Two solutions: $u_{1}, u_{2}$ for $\lambda=3$ (left). Multiple solutions for $\lambda=1.999$ and one solution $u_{c}$ for $\lambda_{c} \approx 3.352731$ (right).

Figures 5.13 and 5.14 wherein a solution of ODE (5.14) for $n=3$ can be identified as a zero of the curve $(u(0), u(1))$. The bifurcation behaviour for $n=3$, is shown in Figure 5.13 at $\tilde{\lambda}=2$. Figure 5.14 provides the bifurcation curves for $n=4,9$ respectively, for different values of $\lambda$.

\section{Experiment 4}

For numerical illustration of the case $n \geq 10$; we take the value $n=10$ as a characteristic example. The numerical results can be found in Figure 5.15. They again confirm the theoretical results as described in section 5.5.3. For this example, we found the critical value $\lambda_{c}=16$. A single solution exists for all values of $\lambda \in\left(0, \lambda_{c}\right)$ and bifurcation diagrams for the dimension parameter $n=10$ are given in Figure 5.15.

\subsection{Conclusion}

In this chapter, we presented a numerical study of the Gelfand-Bratu model for higher dimensions. For three dimensions, we adopted an accurate and efficient nonlinear multigrid approach: the full approximation storage (FAS) extended with a Krylov method as a smoother. In particular, we found new solutions for specific values of the bifurcation parameter. As known from the literature, finding new solutions and new turning points is a hard task. We indeed observed that the three-dimensional case is numerically much more complicated than the oneand two-dimensional cases. Furthermore, we extended the numerical bifurcation curve of the Gelfand-Bratu problem in three dimensions and showed the existence of two new turning points: 

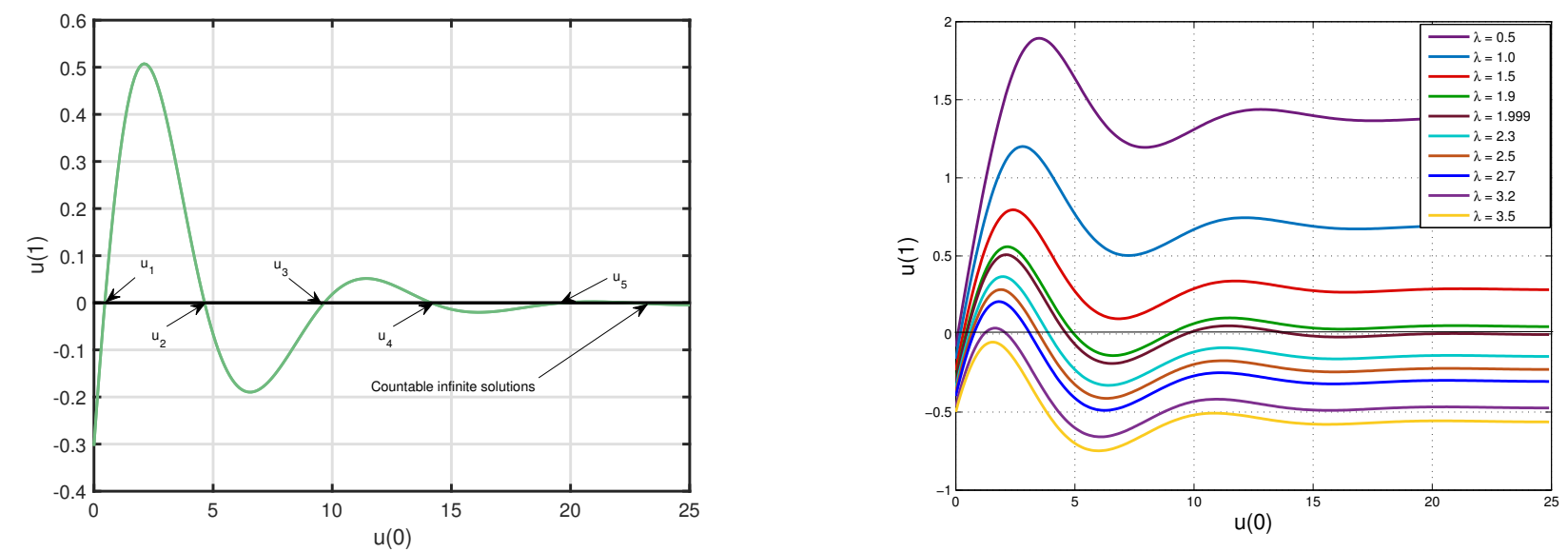

Figure 5.13: Bifurcation curves of model (5.14) for $n=3$ : multiple solutions $u_{1}, u_{2}, u_{3}, u_{4}, u 5$ are identified as a zero of the curve $(u(0), u(1))$ for $\lambda=2$ (left) with different values of $\lambda$ (right).
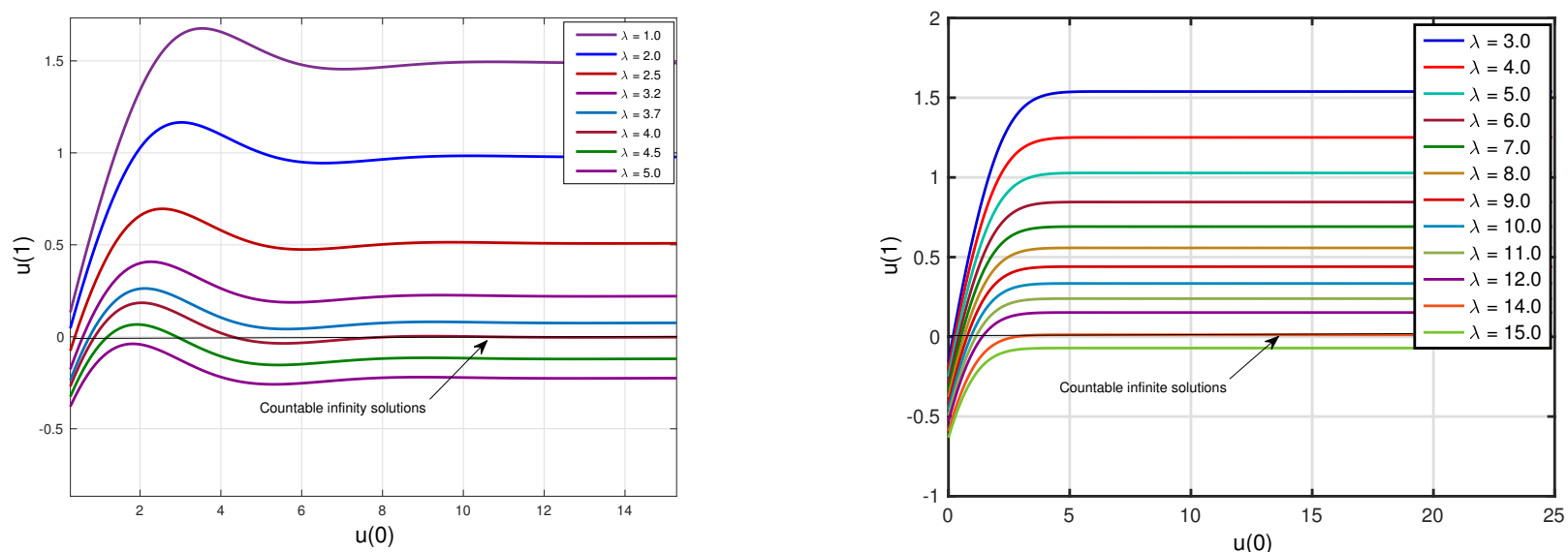

Figure 5.14: Bifurcation curves for $3 \leq n \leq 9$ of nonlinear ODE (5.14): the cases for dimension parameter $n=4$ (left) and $n=9$ (right) respectively, with different values of $\lambda$.
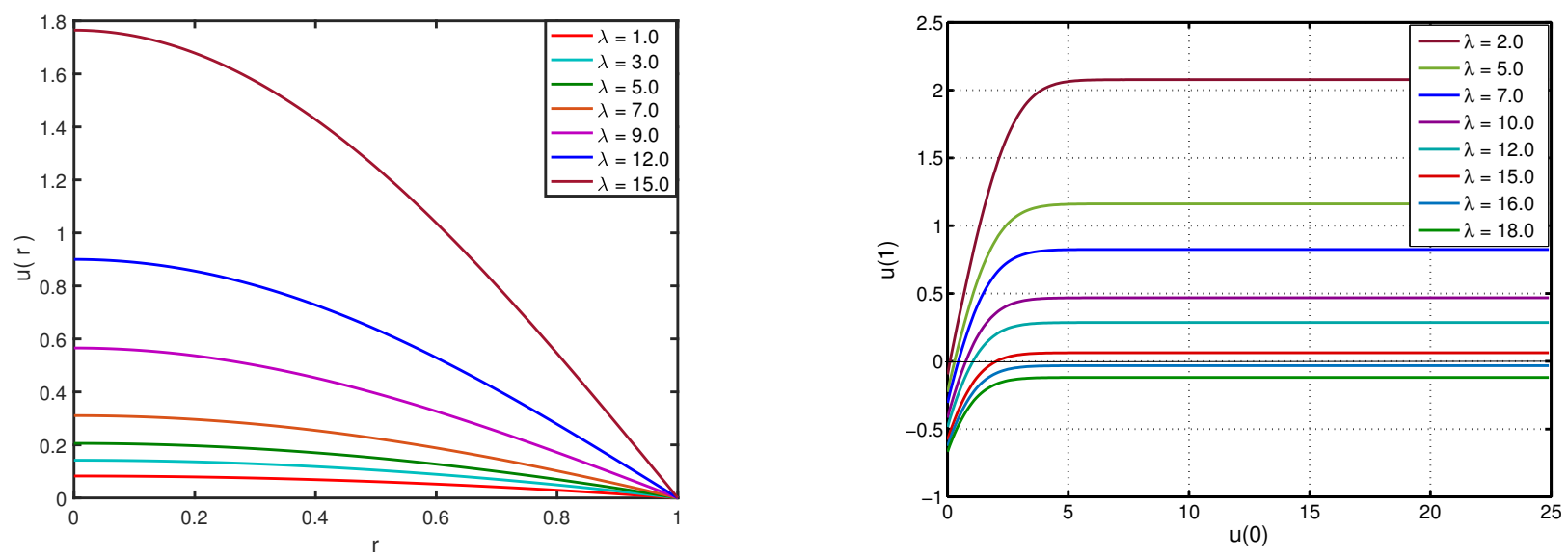

Figure 5.15: Numerical results for dimension parameter $n=10$ of nonlinear model (5.14) with different values of $\lambda$ : one solution (left) and bifurcation curves (right). 
$\lambda_{a}$ and $\lambda_{b}$. Numerical results confirmed the convergence of all types of solutions (unique and multiple) and demonstrated the effectiveness of the proposed numerical strategy for all values of the parameter $\lambda \in\left(0, \lambda_{c}\right]$. For even higher dimensions, we transformed the Gelfand-Bratu problem using $n$-dimensional spherical coordinates to a single nonlinear ODE. We summarized a few theoretical results depending on the dimension parameter $n$ and bifurcation parameter $\lambda$. Numerical solutions of this ODE were computed by a shooting method for a range of values of $n$. The experiments showed the existence of several types of solutions. Bifurcation curves for different values of $n$ and $\lambda$ confirmed the theoretical results of the higher-dimensional GelfandBratu problem as presented in literature. 


\section{Chapter 6}

\section{Conclusions and outlook}

This thesis discusses numerical methods for nonlinear elliptic partial differential equations with parameter dependence such as the Gelfand-Bratu model and singularly perturbed convectiondiffusion-reaction equations. In numerical investigations, more accurate and efficient nonstandard finite difference and multigrid methods are adopted to solve parameter dependent elliptic boundary-value problems.

Aim and objectives of the research are given in chapter 1. Further, it describes the background of Gelfand-Bratu and singularly perturbed problems. In addition, an overview of nonstandard finite difference and multigrid methods is given followed by an outline of the thesis.

In chapter 2, standard and nonstandard finite difference approximations are employed to find the numerical solutions of the one-dimensional truncated Bratu-Picard (BP) model. Numerical results show the existence of infinitely many solutions, which are calculated numerically (for a large, but finite, set of solutions). These new types of solutions are either periodic or semiperiodic. Bifurcation curves of the Bratu-Picard model are provided for each experiment. We observe that the nonstandard finite difference schemes provide more accurate and efficient results than standard finite difference schemes.

In chapter 3, we propose a higher order non-uniform finite difference grid, to solve singularly perturbed boundary value problems with steep boundary-layers. Theoretical properties concerning the extremum values and the asymptotic value at the right boundary point are presented. Several examples of singularly perturbed (linear and nonlinear) convection-diffusion-reaction problems are provided, which demonstrate the effectiveness of the proposed numerical strategy. We establish numerically, not only $4^{\text {th }}$-order but also a $6^{\text {th }}$-order of accuracy by considering only three-point central non-uniform finite differences. Numerical results illustrate that to achieve the $6^{\text {th }}$-order of accuracy, the proposed method needs approximately a factor of 5-10 fewer grid points than the uniform case.

In chapter 4 is about computing numerical solutions of two-dimensional Bratu-type problems. We propose three numerical methods, viz, a finite-difference approximation and two multigrid (MG) approaches: Newton-MG and Full Approximation Storage (FAS)-MG. A comparison, in terms of convergence, accuracy and efficiency among the three numerical methods demonstrate improvement for the whole parameter range $\lambda \in\left(0, \lambda_{c}\right]$. Further, we investigate the bifurcation behaviour of solutions and find new multiplicity of solutions in the case of a cubic approximation of the nonlinear exponential term. We demonstrate that the convergence of all solutions namely, unique, lower, upper, periodic and semi-periodic is obtained for small values of the parameter $\lambda$. Particularly, FAS-MG is found to be more efficient than the other two methods.

In chapter 5, we present a numerical study of the Gelfand-Bratu model for higher dimensions. For three dimensions, we adopt an accurate and efficient nonlinear multigrid approach, namely, FAS-MG extended with a Krylov method as a smoother. New types of solutions are obtained 
for specific values of the bifurcation parameter. Further, the numerical bifurcation curve of the Gelfand-Bratu problem in three dimensions shows the existence of two new turning points. Numerical results confirm the convergence of all types of solutions (unique and multiple) and demonstrate the effectiveness of the proposed numerical strategy. For even higher dimensions, numerical experiments show the existence of several types of solutions. Bifurcation curves confirm the theoretical results of the higher-dimensional Gelfand-Bratu problem as presented in the literature.

\section{Future work}

For future research, the following ideas could be suggested:

- Investigate the stability of the infinite many solutions (periodic and semi-periodic) obtained for the Gelfand-Bratu models in chapters 2, 4 and 5 .

- Explore the bifurcation behaviour of the higher-dimensional Gelfand-Bratu model on more general and more complicated domains.

- Utilize the numerical approach as presented in chapter 3 to find higher order non-uniform grids to solve higher dimensional boundary layer problems.

- Establish a higher order of accuracy by considering five-point central finite differences for a singularly perturbed convection-diffusion-reaction problems.

- Improve FAS-MG extended with Krylov methods to find new types of multiple solutions for three dimensional Gelfand-Bratu model including bifurcation behaviour.

\section{Publications}

- Chapter 2 is published as a transcription of the article [148]: P. A. Zegeling and S. Iqbal, Nonstandard finite differences for a truncated Bratu-Picard model, Journal of Applied Mathematics and Computation, 324: 266-284, 2018.

- Chapter 3 is based on two articles: the first is published as a transcription of the article [58]: S. Iqbal and P. A. Zegeling, Sixth-Order Adaptive Non-uniform Grids for Singularly Perturbed Boundary Value Problems, Numerical Geometry, Grid Generation and Scientific Computing. Lecture Notes in Computational Science and Engineering, Springer, 131: 109124, 2019. The second is published as an article [57]: S. Iqbal and P. A. Zegeling, Higher order non-uniform grids for singularly perturbed convection-diffusion-reaction problems, Journal of Computational Mathematics and Mathematical Physics, Springer, 2019, 59(12): Springer, 131: 109-124, 2019.

- Chapter 4 is in press as an article [60]: S. Iqbal and P. A. Zegeling, An efficient nonlinear multigrid scheme for two-dimensional boundary value problems, Journal of Applied Mathematics and Computation, 2020.

- Chapter 5 is in press as a transcription of an article [59]: S. Iqbal and P. A. Zegeling, A numerical study of the higher-dimensional Gelfand-Bratu model, Journal of Computers and Mathematics with Applications, 2020. 


\section{Bibliography}

[1] S. Abbasbandy, M. S. Hashemi, and C. Liu. The Lie-group shooting method for solving the Bratu equation. Communications in Nonlinear Science and Numerical Simulation, 16(11):4238-4249, 2011.

[2] Z. Aganović and Z. Gajić. Linear Optimal Control of Bilinear Systems with Applications to Singular Perturbations and Weak Coupling, volume 206 of Lecture Notes in Control and Information Sciences. Springer-Verlag, Berlin Heidelberg, 1995.

[3] R. P. Agarwal, D. O'Regan, and P. J. Y. Wong. Positive Solutions of Differential, Difference and Integral Equations. Springer Science and Business Media, 1998.

[4] A. K. Agrawal and R. S. Peckover. Nonuniform grid generation for boundary-layer problems. Computer Physics Communications, 19(2):171-178, 1980.

[5] U. M. Ascher, R. M. M. Mattheij, and R. D. Russell. Numerical Solution of Boundary Value Problems for Ordinary Differential Equations, volume 13. SIAM, Philadelphia, 1994.

[6] N. S. Bakhvalov. The optimization of methods of solving boundary value problems with a boundary layer. USSR Computational Mathematics and Mathematical Physics, 9(4): 139-166, 1969.

[7] T. Bakri, Y. A. Kuznetsov, F. Verhulst, and E. Doedel. Multiple solutions of a generalized singular perturbed Bratu problem. International Journal of Bifurcation and Chaos, 22(4), 2012.

[8] R. Barrett, M. Berry, T. F. Chan, J. Demmel, J. Donato, J. Dongarra, V. Eijkhout, R. Pozo, C. Romine, and H. V. der Vorst. Templates for the Solution of Linear Systems: Building Blocks for Iterative Methods, volume 43. SIAM, Philadelphia, 1994.

[9] B. Batiha. Numerical solution of Bratu-type equations by the variational-iteration method. Hacettepe Journal of Mathematics and Statistics, 39(1):23-29, 2010.

[10] R. Beauwens. Iterative solution methods. Applied Numerical Mathematics, 51(4):437-450, 2004.

[11] J. Bebernes and D. Eberly. Mathematical Problems from Combustion Theory, volume 83 of Applied Mathematical Sciences. Springer Science and Business Media, 2013.

[12] G. Beckett and J. A. Mackenzie. On a uniformly accurate finite difference approximation of a singularly perturbed reaction-diffusion problem using grid equidistribution. Journal of Computational and Applied Mathematics, 131(1-2):381-405, 2001.

[13] J. P. Boyd. An analytical and numerical study of the two dimensional Bratu problem. Journal of Scientific Computing, 1(2):183-206, 1986. 
[14] J. P. Boyd. One-point pseudospectral collocation for the one dimensional Bratu equation. Applied Mathematics and Computation, 217(12):5553-5565, 2011.

[15] A. Brandt. Multi-level adaptive solutions to boundary value problems. Mathematics of computation, 31(138):333-390, 1977.

[16] A. Brandt, S. McCormick, and J. Ruge. Multigrid methods for differential eigenproblems. SIAM Journal on Scientific and Statistical Computing, 4(2):244-260, 1983.

[17] G. Bratu. Sur l'équations intégrales exponentielle. Comptes Rendus des Seances de l'Academie des Sciences, pages 1048-1050, 1911.

[18] G. Bratu. Sur les équations intégrales non linéaires. Bulletin de la Société Mathématique de France, 42:113-142, 1914.

[19] R. R. Brown. Numerical solution of boundary value problems using nonuniform grids. Journal of the Society for Industrial and Applied Mathematics, 10(3):475-495, 1962.

[20] R. Buckmire. Investigations of nonstandard, Mickens-type, finite difference schemes for singular boundary value problems in cylinderical or spherical coordinates. Numerical Methods for Partial Differential Equations: An International Journal, 19(3):380-398, 2003.

[21] R. Buckmire. Application of a Mickens finite-difference scheme to the cylindrical BratuGelfand problem. Numerical Methods for Partial Differential Equations: An International Journal, 20(3):327-337, 2004.

[22] C. J. Budd, W. Huang, and R. D. Russell. Adaptivity with moving grids. Acta Numerica, 18:111-241, 2009.

[23] T. F. C. Chan and H. B. Keller. Arc-length continuation and multigrid techniques for nonlinear elliptic eigenvalues problems. SIAM Journal on Scientific and Statistical Computing, 3(2):173-194, 1982.

[24] S. Chandrasekhar. An Introduction to the Study of Stellar Structure, volume 2. Dover Publications, INC, 1967.

[25] Z. Chen, A. B. Gumel, and R. E. Mickens. Nonstandard discretizations of the generalized nagumo reaction-diffusion equation. Numerical Methods for Partial Differential Equations, 19(3):363-379, 2003.

[26] W. C. Connett, W. L. Golik, and A. L. Schwartz. Superconvergent grids for two-point boundary value problems. Mathematica Aplicada e Computacional, 10:43-58, 1991.

[27] N. Das, R. Singh, A. M. Wazwaz, and J. Kumar. An algorithm based on the variational iteration technique for the Bratu-type and the Lane-Emden problems. Journal of Mathematical Chemistry, 54(2):527-551, 2016.

[28] P. Das and V. Mehrmann. Numerical solution of singularly perturbed convection-diffusionreaction problems with two small parameters. BIT Numerical Mathematics, 56(1):51-76, 2016 .

[29] P. de Oliveira and F. A. Oliveira. On a theoretical justification of adaptive gridding for finite difference approximations. In R. P. Agarwal, Y. M. Chow, and S. J. Wilson, editors, Numerical Mathematics Singapore, volume 86 of International Series of Numerical Mathematics, pages 391-401. Birkhäuser, Basel, 1988. 
[30] E. K. de Rivas. On the use of non-uniform grids in finite difference equations. Journal of Computational Physics, 10(2):202-210, 1972.

[31] L. M. Degtyarev, V. V. Prozdov, and T. S. Ivanova. Mesh method for singularly perturbed one dimensional boundary value problems, with a mesh adapting to the solution. Differential Equations, 23(7):772-779, 1987.

[32] Y. Du. Exact multiplicity and S-shaped bifurcation curve for some semilinear elliptic problems from combustion theory. SIAM Journal on Mathematical Analysis, 32(4):707733,2000 .

[33] W. Eckhaus. Asymptotic Analysis of Singular Perturbations, volume 9 of Studies in Mathematics and its Applications. Elsevier, 2011.

[34] W. Eckhaus. Matched Asymptotic Expansions and Singular Perturbations, volume 6 of North-Holland Mathematics Studies. Elsevier, 2011.

[35] U. Erdogan and T. Ozis. A smart nonstandard finite difference scheme for second order nonlinear boundary value problems. Journal of Computational Physics, 230(17):6464$6574,2011$.

[36] R. P. Fedorenko. A relaxation method for solving elliptic difference equations. USSR Computational Mathematics and Mathematical Physics, 1(4):1092-1096, 1962.

[37] R. P. Fedorenko. The speed of convergence of one iterative process. USSR Computational Mathematics and Mathematical Physics, 4(3):227-235, 1964.

[38] D. A. Frank-Kamenetskii. Diffusion and Heat Exchange in Chemical Kinetics. Princeton University Press, 1955.

[39] K. O. Friedrichs and W. R. Wasow. Singular perturbations of non-linear oscillations. Duke Mathematical Journal, 13(3):367-381, 1946.

[40] E. C. Gartland. Graded-mesh difference schemes for singularly perturbed two-point boundary value problems. Mathematics of computation, 51(184):631-657, 1988.

[41] G. R. Gavalas. Nonlinear Differential Equations of Chemically Reacting Systems, volume 17 of Springer Tracts in Natural Philosophy. Springer-Verlag, Berlin Heidelberg, 1968.

[42] I. M. Gelfand. Some problems in the theory of quasi-linear equations. Uspekhi Matematicheskikh Nauk, 14(2):87-158, 1959.

[43] B. Gidas, W. Ni, and L. Nirenberg. Symmetry and related properties via the maximum principle. Communications in Mathematical Physics, 68(3):209-243, 1979.

[44] J. Goodman and A. D. Sokal. Multigrid Monte Carlo for lattice field theories. Physical review letters, 56(10):1015, 1986.

[45] A. B. Gumel, S. M. Maghadas, and R. E. Mickens. Effect of a preventive vaccine on the dynamics of HIV transmission. Communications in nonlinear science and numerical simulation, 9(6):649-659, 2004.

[46] W. Hackbusch. On the computation of approximate eigenvalues and eigenfunctions of elliptic operators by means of multigrid methods. SIAM Journal on Numerical Analysis, 16(2):201-215, 1979. 
[47] W. Hackbusch. Multi-Grid Methods and Applications, volume 4 of Computational Mathematics. Springer-Verlag, Berlin Heidelberg, 1985.

[48] W. Hackbusch. Comparison of different multi-grid variants for nonlinear equations. ZAMM-Journal of Applied Mathematics and Mechanics, 72(2):148-151, 1992.

[49] W. Hackbusch. Iterative Solutions of Large Sparse Systems of Equations, volume 95 of Applied Mathematical Sciences. Springer-Verlag New York, 1994.

[50] W. Hackbusch and U. Trottenberg, editors. Multigrid Methods, volume 960 of Proceedings of the conference held at Köln-Porz. Springer-Verlag, Berlin Heidelberg, 1982.

[51] M. Hajipour, A. Jajarmi, and D. Baleanu. On the accurate discretization of a highly nonlinear boundary value problem. Numerical Algorithms, 79(3):679-695, 2018.

[52] S. P. Hastings and J. B. McLeod. Classical Methods in Ordinary Differential Equations: With Applications to Boundary Value Problems, volume 129 of Graduate Studies in Mathematics. American Mathematical Society, 2011.

[53] R. D. Haynes, W. Huang, and P. A. Zegeling. A numerical study of blowup in the harmonic map heat flow using the MMPDE moving mesh method. Numerical Mathematics: Theory, Methods and Applications, 6(2):364-383, 2013.

[54] J. D. Hoffman. Relationship between the truncation errors of centred finite-difference approximations on uniform and non-uniform meshes. Journal of Computational Physics, 46(3):469-474, 1982.

[55] M. H. Holmes. Introduction to Perturbation Methods, volume 20 of Texts in applied mathematics. Springer-Verlag New York, 1995.

[56] F. D. Hoog and D. Jackett. On the rate of convergence of finite difference schemes on non-uniform grids. ANZIAM Journal, 26(3):247-256, 1985.

[57] S. Iqbal and P. A. Zegeling. Higher order non-uniform grids for singularly perturbed convection-diffusion-reaction problems. Journal of Computational Mathematics and Mathematical Physics, 59(12):2057-2079, 2019.

[58] S. Iqbal and P. A. Zegeling. Sixth-order adaptive non-uniform grids for singularly perturbed boundary value problems. Numerical Geometry, Grid Generation and Scientific Computing, Lecture Notes in Computational Science and Engineering, Springer, 131:109$124,2019$.

[59] S. Iqbal and P. A. Zegeling. A numerical study of the higher-dimensional Gelfand-Bratu model. Journal of Computers and Mathematics with Applications (In Press), 2020.

[60] S. Iqbal and P. A. Zegeling. An efficient nonlinear multigrid scheme for two-dimensional boundary value problems. Journal of Applied Mathematics and Computation (In Press), 2020 .

[61] J. Jacobsen and K. Schmitt. The Liouville-Bratu-Gelfand Problem for radial operators. Journal of Differential Equations, 184(1):283-298, 2002.

[62] I. Jacques and C. Judd. Numerical Analysis. Chapman and Hall Ltd, 1987.

[63] F. Jing-Jing, H. Ling, and Y. Shi-Jie. Solutions of Laplace equation in n-dimensional spaces. Communications in Theoretical Physics, 56(4):623-625, 2011. 
[64] D. D. Joseph and T. S. Lundgren. Quasilinear Dirichlet problems driven by positive sources. Archive for Rational Mechanics and Analysis, 49(4):241-269, 1973.

[65] T. Kalkreuter. Multigrid methods for the computation of propagators in gauge fields. International Journal of Modern Physics, 5(4):629-700, 1994.

[66] J. Karkowski. Numerical experiments with the Bratu equation in one, two and three dimensions. Computational and Applied Mathematics, 32(2):231-244, 2013.

[67] T. Kashiwagi. A radiative ignition model of a solid fuel. Journal of Combustion Science and Technology, 8(5-6):225-236, 1973.

[68] A. Kaya. Finite difference approximations of multidimensional unsteady convectiondiffusion-reaction equations. Journal of Computational Physics, 285:331-349, 2015.

[69] A. Kaya and A. Sendur. Finite difference approximations of multidimensional convectiondiffusion-reaction problems with small diffusion on a special grid. Journal of Computational Physics, 300:574-591, 2015.

[70] H. B. Keller. Numerical methods in boundary-layer theory. Annual Review of Fluid Mechanics, 10(1):417-433, 1978.

[71] G. Khakimzyanov and D. Dutykh. On superconvergence phenomenon for second order centered finite differences on non-uniform grids. Journal of Computational and Applied Mathematics, 326:1-14, 2017.

[72] A. M. Khodier. A finite difference scheme on non-uniform grids. International Journal of Computer Mathematics, 77(1):145-152, 2001.

[73] P. Korman, Y. Li, and T. Ouyang. Exact multiplicity results for boundary value problems with nonlinearities generalizing cubic. Proceedings of the Royal Society of Edinburgh ection A: Mathematics, 126(3):599-616, 1996.

[74] D. Kumar. A parameter-uniform method for singularly perturbed turning point problems exhibiting interior or twin boundary layers. International Journal of Computer Mathematics, 96(5):865-882, 2019.

[75] V. Kumar and B. Srinivasan. An adaptive mesh strategy for singularly perturbed convection diffusion problems. Applied Mathematical Modelling, 39(7):2081-2091, 2015.

[76] T. Laetsch and J. B. Keller. The number of solutions of a nonlinear two point boundary value problem. Indiana University Mathematics Journal, 20(1):1-13, 1970.

[77] R. J. LeVeque. Finite Difference Methods for Ordinary and Partial Differential Equations. SIAM, Philadelphia, 1955.

[78] W. L. Lick. Difference equations from differential equations, volume 41 of Lecture notes in engineering. Springer-Verlag, Berlin Heidelberg, 1989.

[79] V. D. Liseikin. Grid generation methods. Springer Science and Business Media, 2010.

[80] X. Liu, G. R. Liu, K. Tai, and K. Y. Lam. Radial point interpolation collocation method (RPICM) for the solution of nonlinear Poisson problems. Computational Mechanics, 36(4): 298-306, 2005. 
[81] G. Mack and S. Meyer. The effective action from multigrid Monte Carlo. Nuclear Physics B-Proceedings Supplements, 17(293-296), 1990.

[82] S. F. McCormick. Multigrid Methods. SIAM, Philadelphia, 1987.

[83] J. S. McGough. Numerical continuation and the Gelfand problem. Applied Mathematics and Computation, 89(1-3):225-239, 1998.

[84] R. E. Mickens. Difference equation models of differential equations having zero local truncation error, volume 92 of North-Holland Mathematics Studies, pages 445-449. Elsevier Science Publishers B.V., 1984.

[85] R. E. Mickens. Exact solutions to a finite difference model of a nonlinear reaction-advection equation: implications for numerical analysis. Numerical Methods for Partial Differential Equations, 5(4):313-325, 1989.

[86] R. E. Mickens. Difference Equations: Theory and Applications. Chapman and Hall, New York, 1990.

[87] R. E. Mickens. Nonstandard finite difference models of differential equations. World Scientific, 1993.

[88] R. E. Mickens. A best finite-difference scheme for the Fisher equation. Numerical Methods for Partial Differential Equations, 10(5):581-585, 1994.

[89] R. E. Mickens. Applications of Nonstandard Finite Difference Schemes. World Scientific Publishing Co.Pte.Ltd, 2000.

[90] R. E. Mickens. Nonstandard finite difference schemes for differential equations. Journal of Difference Equations and Applications, 8(9):823-847, 2002.

[91] R. E. Mickens and P. M. Jordan. A positivity-preserving nonstandard finite difference scheme for the damped wave equation. Numerical Methods for Partial Differential Equations: An International Journal, 20(5):639-649, 2004.

[92] R. E. Mickens and A. Smith. Finite difference models of ordinary differential equations: influence of denominator functions. Journal of the Franklin Instituite, 327(1):143-145, 1990.

[93] J. J. H. Miller, E. O'Riordan, and G. I. Shishkin. Fitted Numerical Methods for Singular Perturbation Problems: Error Estimates in the Maximum Norm for Linear Problems in One and Two Dimensions. World Scientific Publishing Co., 1996.

[94] H. D. Mittelmann. Multilevel continuation technique for nonlinear boundary value problems with parameter dependence. Applied Mathematics and Computation, 19(1-4):265-282, 1986.

[95] H. D. Mittelmann and H. Weber. Multi-grid solution of bifurcation problems. SIAM Journal on Scientific and Statistical Computing, 6(1):49-60, 1985.

[96] A. Mohsen. A simple solution of the Bratu problem. Computers and Mathematics with Applications, 67(1):26-33, 2014.

[97] A. Mohsen, L. F. Sedeek, and S. A. Mohamed. New smoother to enhance multigrid-based methods for the Bratu problem. Applied Mathematics and Computation, 204(1):325-339, 2008 . 
[98] G. Moore and A. Spence. The calculation of turning points of nonlinear equations. SIAM Journal on Numerical Analysis, 17(4):567-576, 1980.

[99] A. S. Mounim and B. M. D. Dormale. From the fitting technique to accurate schemes for the Liouville-Bratu-Gelfand problem. Numerical Methods for Partial Differential Equations, 22(4):761-775, 2006.

[100] W. A. Mulder. A new multigrid approach to convection problems. Journal of Computational Physics, 83(2):303-323, 1989.

[101] P. D. Oliveira. On the characterization of finite difference "optimal" meshes. Journal of Computational and Applied Mathematics, 36:137-148, 1991.

[102] R. E. O'Malley. Phase-plane solutions to some singular perturbation problems. Journal of Mathematical Analysis and Applications, 54(2):449-466, 1976.

[103] R. E. O'Malley. Singular Perturbation Methods for Ordinary Differential Equations, volume 89 of Applied Mathematical Sciences. Springer-Verlag, New York, 1991.

[104] C. W. Oosterlee and P. Wesseling. A multigrid method for an invariant formulation of the incompressible navier-stokes equations in general co-ordinates. Communications in applied numerical methods, 8(10):721-734, 1992.

[105] J. M. Ortega and W. C. Rheinboldt. Iterative Solution of Nonlinear Equations in Several Variables, volume 30 of Classics in Applied Mathematics. SIAM, Philadelphia, 1970.

[106] M. Özişik. Finite Difference Methods in Heat Transfer. CRC Press, Inc., 1994.

[107] E. Picard. Sur certains exemples singuliers d'approximations successives. Comptes rendus de l'Académie des Sciences, 126:497-500, 1898.

[108] L. Prandtl. Über Flüssigkeits-bewegung bei kleiner Reibung. In Verhandlungen. III. Internationalen Mathematiker Kongresses, pages 484-491, 1905.

[109] A. Quarteroni and A. Valli. Numerical Approximation of Partial Differential Equations, volume 23 of Springer Series in Computational Mathematics. Springer Science and Business Media, 2008.

[110] O. Ragb, L. F. Seddek, and M. S. Matbuly. Iterative differential quadrature solutions for Bratu problem. Computers and Mathematics with Applications, 74(2):249-257, 2017.

[111] M. P. Rajan and G. D. Reddy. A regularized iterative scheme for solving singularly perturbed elliptic PDE. Mathematics and Computers in Simulation, 144:21-34, 2018.

[112] J. Rashidinia, K. Maleknejad, and N. Taheri. Sinc-Galerkin method for numerical solution of Bratu's problem. Numerical Algorithms, 62(1):1-11, 2013.

[113] G. O. Roberts. Computational meshes for boundary layer problems. In Proceedings of the Second International Conference on Numerical Methods in Fluid Dynamics, volume 8 of Lecture Notes in Physics, pages 171-177. Springer-Verlag, Berlin Heidelberg, 1971.

[114] H. G. Roos, M. Stynes, and L. Tobiska. Numerical Methods for Singularly Perturbed Differential Equations: Convection-Diffusion and Flow Problems, volume 24 of Springer Series in Computational Mathematics. Springer-Verlag, Berlin Heidelberg, 1996.

[115] Y. Saad. Iterative Methods for Sparse Linear Systems. SIAM, Philadelphia, 2003. 
[116] J. A. Schetz. Foundations of Boundary Layer Theory for Momentum, Heat, and Mass Transfer. Inc. Englewood Cliffs: Prentice-Hall, 1984.

[117] H. Schlichting and K. Gersten. Boundary Layer Theory. Springer-Verlag, Berlin Heidelberg, 9 edition, 2016.

[118] M. Shashkov. Conservative Finite-Difference Methods on General Grids. CRC press, 2018.

[119] G. I. Shishkin and L. P. Shishkina. Difference Methods for Singular Perturbation Problems. Chapman and Hall/CRC, 2008.

[120] I. Shufrin, O. Rabinovitch, and M. Eisenberger. Elastic nonlinear stability analysis of thin rectangular plates through a semi-analytical approach. International Journal of Solids and Structures, 46(10):2075-2092, 2009.

[121] Z. Sorbjan. Structure of the Atmospheric Boundary Layer. Prentice Hall, 1989.

[122] J. C. Strikwerda. Finite Difference Schemes and Partial Differential Equations. SIAM, Philadelphia, 2004.

[123] K. Stüben. Algebraic Multigrid (AMG): An Introduction with Applications. GMDForschungszentrum Informationstechnik, 1999.

[124] K. Stüben and U. Trottenberg. Multigrid methods: Fundamental algorithms, model problem analysis and applications. In Multigrid Methods, volume 960 of Lecture Notes in Mathematics, pages 1-176. Springer-Verlag, Berlin Heidelberg, 1982.

[125] H. Sundqvist and G. Veronis. A simple finite difference grid with non-constant intervals. Tellus, 22(1):26-31, 1970.

[126] M. I. Syam and B. S. Attili. Weighted residual method for obtaining positive solutions of two point nonlinear boundary value problems. Applied Mathematics and Computation, 176(2):775-784, 2006.

[127] S. Ta'asan. Multigrid methods for locating singularities in bifurcation problems. SIAM Journal on Scientific and Statistical Computing, 11(1):51-62, 1990.

[128] H. Temimi and M. Ben-Romdhane. An iterative finite difference method for solving Bratu's problem. Journal of Computational and Applied Mathematics, 292:76-82, 2016.

[129] J. Thomas. Numerical Partial Differential Equations: Finite Difference Methods, volume 22 of Texts in Applied Mathematics. Springer-Verlag, New York, 1995.

[130] A. N. Tikhonov. Systems of differential equations containing small parameters multiplying some of the derivatives. Matematicheskii sbornik, 73(3):575-586, 1952.

[131] U. Trottenberg, C. W. Oosterlee, and A. Schuller. Multigrid. Academic Press, 2000.

[132] S. Vandewalle and R. Piessens. Efficient parallel algorithms for solving initial boundary value and time-periodic parabolic partial differential equations. SIAM Journal of Scientific and Statistical Computing, 13(6):1330-1346, 1992.

[133] A. B. Vasil'eva. Asymptotic behavior of solutions to certain problems involving nonlinear ordinary differential equations containing a small parameter multiplying the highest derivatives. Russian Mathematical Surveys, 18(3):13-84, 1963. 
[134] A. E. P. Veldman and K. Rinzema. Playing with non-uniform grid. Journal of Engineering Mathematics, 26(1):119-130, 1992.

[135] F. Verhulst. Methods and Applications of Singular Perturbations: Boundary Layers and Multiple Timescale Dynamics, volume 50 of Texts in Applied Mathematics. Springer Science and Business Media, 2005.

[136] M. Vinokur. On one dimensional stretching functions for finite difference calculations. Journal of Computational Physics, 50(2):215-234, 1983.

[137] Y. Q. Wan, Q. Guo, and N. Pan. Thermo-electro-hydrodynamic model for electrospinning process. International Journal of Nonlinear Sciences and Numerical Simulation, 5(1):5-8, 2004.

[138] T. Washio and C. Oosterlee. Krylov subspace accerlation for nonlinear multigrid schemes. Electronic Transactions on Numerical Analysis., 6:271-290, 1997.

[139] A. M. Wazwaz. Adomian's decomposition method for a reliable treatment of the Bratutype equations. Applied Mathematics and Computation, 166(3):652-663, 2005.

[140] P. Wesseling. A robust and efficient multigrid method. In W. Hackbusch and U. Trottenberg, editors, Multigrid Methods, volume 960 of Lecture Notes in Mathematics, pages 614-630. Springer-Verlag, Berlin Heidelberg, 1982.

[141] P. Wesseling. Finite volume and finite difference discretization on nonuniform grids, volume 29 of Springer Series in Computational Mathematics, pages 81-109. SpringerVerlag, Berlin Heidelberg, 2009.

[142] P. Wesseling and C. W. Oosterlee. Geometric multigrid with applications to computational fluid dynamics. Journal of Computational and Applied Mathematics, 128(1-2):311-334, 2001.

[143] N. K. Yamaleev. Minimization of the truncation error by grid adaptation. Journal of Computational Physics, 170(2):459-497, 2001.

[144] Z. Yang and S. Liao. A HAM-based wavelet approach for nonlinear partial differential equations: two dimensional Bratu problem as applications. Communications in Nonlinear Science and Numerical Simulation, 53:249-262, 2017.

[145] V. F. Zaitsev and A. D. Polyanin. Handbook of Exact Solutions for Ordinary Differential Equations. Chapman and Hall/CRC, 2002.

[146] A. Zegeling and P. A. Zegeling. S-shaped bifurcations in a quadratic Hamiltonian system. in preparation, 2019.

[147] P. A. Zegeling. Theory and Application of Adaptive Moving Grid Methods, chapter 7, pages 251-293. Adaptive Computations: Theory and Algorithms. Science Press, Peking, 2007.

[148] P. A. Zegeling and S. Iqbal. Nonstandard finite differences for a truncated Bratu-Picard model. Applied Mathematics and Computation, 324:266-284, 2018.

[149] P. A. Zegeling, I. Lagzi, and F. Izsak. Transition of Liesegang precipitation systems: simulations with an adaptive grid PDE method. Communications in Computational Physics, 10(4):867-881, 2011. 



\section{Samenvatting}

Dit proefschrift behandelt numerieke methoden voor niet-lineaire elliptische partiële differentiaalvergelijkingen met parameter-afhankelijkheid. Voorbeelden hiervan zijn het GelfandBratu model en singulier gestoorde convectie-diffusie-reactievergelijkingen. Bij dit onderzoek worden nauwkeurigere en efficiëntere niet-uniforme, en niet-standaard, eindige-differentie methoden en niet-lineaire multigrid-methoden ontwikkeld en getest om parameter-afhankelijke elliptische randwaardeproblemen numeriek te benaderen.

Hoofdstuk 1 verschaft duidelijkheid over de doelstellingen van het onderliggende onderzoek. De achtergrond van het Gelfand-Bratu model en singulier gestoorde problemen worden in het kort beschreven. Daarnaast wordt er een overzicht gegeven van niet-standaard eindige-differentie en multigrid-methoden, gevolgd door een overzicht en indeling van het proefschrift.

In hoofdstuk 2 worden standaard en niet-standaard eindige-differentie benaderingen gebruikt voor het bepalen van numerieke oplossingen van een ééndimensionaal Bratu-Picard model. Theoretische eigenschappen van dit model worden beschreven waaronder de symmetrie, het asymptotische gedrag en de uniciteit van de oplossingen. Numerieke resultaten voorspellen het bestaan van een oneindig aantal oplossingen in bepaalde gevallen. Dit type oplossingen kan periodiek of semi-periodiek zijn. Voor alle mogelijke situaties worden bifurcatiekrommen van het Bratu-Picard model gegeven. Verder zien we dat de niet-standaard eindige-differentie schema's nauwkeuriger en efficiënter blijken te zijn dan standaard eindige differenties.

In hoofdstuk 3 introduceren we een hogere orde niet-uniform eindige-differentie rooster om singulier gestoorde randwaardeproblemen met grenslagen door te rekenen. Theoretische resultaten betreffende de extremumwaarden en het asymptotische gedrag aan de rechterrand worden gepresenteerd. Meerdere voorbeelden van singulier gestoorde (lineaire en niet-lineaire) convectiediffusie-reactieproblemen worden behandeld, die de effectiviteit van de voorgestelde numerieke strategie bevestigen. We stellen numeriek vast, dat we niet alleen een vierde orde (i.p.v. een tweede orde), maar zelfs een zesde orde nauwkeurigheid kunnen bewerkstelligen door slechts drie roosterpunten te nemen in een centrale niet-uniforme benadering. Dit alles door het slim kiezen van een speciale roostertransformatie in termen van een equidistributieprincipe. Numerieke experimenten illustreren het zesde orde gedrag. De voorgestelde methode heeft ongeveer een factor 5 à 10 minder roosterpunten nodig dan een uniform rooster met dezelfde nauwkeurigheid.

Hoofdstuk 4 gaat over het berekenen van numerieke oplossingen van tweedimensionale problemen van het Bratu type. We stellen drie numerieke methoden voor, namelijk een eindigedifferentiebenadering en twee typen multigrid (MG) benaderingen: Newton-MG en Full Approximation Storage (FAS)-MG. Een vergelijking tussen de drie numerieke methoden, in termen van convergentie, nauwkeurigheid en efficiëntie, toont een duidelijk verschil aan voor het hele parameterbereik in het model. Verder onderzoeken we het bifurcatiegedrag van de verschillende oplossingen en vinden we, net als in 1d, nieuwe soorten oplossingen in het geval van een derdegraadsbenadering van de niet-lineaire exponentiële term. We observeren numerieke convergentie voor alle oplossingen, zowel voor het geval van unieke als periodieke en semi-periodieke oplossingen. In het bijzonder blijkt FAS-MG veel efficiënter te zijn dan de andere twee genoemde 
methoden.

In hoofdstuk 5 presenteren we een numerieke studie van het Gelfand-Bratu model voor hogere ruimtedimensies. In drie dimensies passen we een nauwkeurige en efficiënte niet-lineaire multigridmethode toe, namelijk de eerdergenoemde FAS-MG, nu uitgebreid met een Krylovmethode als een zogenaamde 'gladmaker'. Nieuwe typen oplossingen worden verkregen voor specifieke waarden van de bifurcatieparameter. Verder vinden we een uitgebreidere numerieke bifurcatiekromme van het Gelfand-Bratu probleem dan tot nu toe bekend, met twee nieuwe 'turningpoints'. Voor nog hogere dimensies $(>3)$ tonen numerieke experimenten het bestaan van een grote verscheidenheid aan oplossingen. Bifurcatiekrommen bevestigen de theoretische resultaten uit de literatuur van het hoger-dimensionale Gelfand-Bratu probleem. 


\section{Acknowledgements}

First and foremost, I would like to express my gratitude towards my promotor Rob Bisseling and my co-promotor Paul Zegeling for their expert guidance and support throughout my Ph.D. Paul, I would never forget your open-door policy, our many long meetings and talks; thank you for your guidance and effort in me. I enjoyed working together with you because of your experience, guidance, patience, and support. Rob, thanks for being my promotor and unflagging support during this four years' journey.

I am very grateful to the members of my thesis reading committee, Prof. Chris Budd, Prof Christian Rohde, Prof. Barry Koren, Prof. Kees Oosterlee and Dr. Lenard Kamenski for their time, effort, valuable comments and suggestions. I really appreciate your feedback.

I would like to thank my current and former office mates, Hong, Dominik, Sjors, Marieke, Ajinkya, Arjen, Inan, Han, Ralph and Bart for their kindness. Special thanks go to Felix for the informative discussions, suggestions and encouragement.

I would like to thank André Zegeling and Prof. Ferdinand Verhulst for interesting discussions and for their valuable comments. I am especially grateful to Barbara, Cécile, Wilke, Jean, Ria, and Sylvia, and to the other staff members at the Mathematical Institute for their sincere assistance and kindness during my stay in the Utrecht University. I give special thanks to Gunther, Jason, Carin, Eva Marije and Martijn for their support in resolving my kids daycare and education during this busy period.

I am pleased to have this opportunity to thank the Schlumberger Foundation for awarding me the Faculty for Future Award to do my doctoral studies. The financial support of Schlumberger has been invaluable in completing my Ph.D. research. I am grateful to Ms. Regina Hand and Ms. Eve Millon for taking care of things and ensuring everything runs smoothly.

Then, I would like to very warmly thank my parents (Ammi and Abbu), my siblings (Imrana Api, Amir Bhai, Saima Api, Adnan Bhai and my lovely younger brother Imran), my lovely nephews (Mutahir, Ayan and Mustafa) and my sweet niece Wareesha for their ever-lasting support, love, kindness, and understanding. I appreciate my lovely kids Razeen, Bameen and my little one Yazaan for abiding my ignorance and the patience, they showed during mama's Ph.D. research.

Finally, I am deeply indebted and grateful to my husband, who is the love of my life, Tariq, for keeping things going and for always showing how proud he is of me. He has always given me the extra strength and motivation to get things done. 



\section{Curriculum Vitae}

Sehar Iqbal was born on 29th November, 1983 in Faisalabad one of the industrial cities of Pakistan. She was raised in the capital Islamabad, where she obtained her elementary and secondary school education. Afterwards she secured a scholarship to do her Bachelor in Mathematics from COMSATS Institute of Information Technology (now COMSATS University), Islamabad. She graduated in 2006 with her final research project in the field of fluid dynamics on the topic "An exploratory study was conducted to understand the formation of Taylor columns in rotating fluids ". She participated in several conferences during her bachelor studies.

After her Bachelor she started to work as a teaching assistant in Air University, Islamabad for one year, where she was responsible for tutorials of the bachelor mathematics courses. In 2007, she moved to COMSATS university, Islamabad to work as a lecturer. She worked as a lecturer for two years to teach at the bachelor level. During the same period she also did her Master's in Applied Mathematics from the same university. She concluded her Master in 2007 with a research carried out to "investigate and explore the Guided Waves (Dispersion curves and relations) in a Fluid-loaded Orthotropic Plate for both symmetric and anti-symmetric modes". Later she published some research articles on the same topic. There she was supervised by Prof. Aftab Khan. In 2009 she got fully funded International Scholarship from University of Twente, The Netherlands, where in 2012 she completed her second Master degree in Mathematical Physics and Computational Mechanics. The Master thesis research was conducted with Prof. Hans Zwart on the topic to "Investigate the Infinite Dimensional System Theory for Networks of Flows".

In 2012, she went back to Pakistan to continue her job as a Lecturer in the Mathematics department, COMSATS University. In 2015, she was awarded a doctoral research funding from Schlumberger (international oilfield services company) under the Faculty for future program. She started as a PhD candidate at the Mathematics Department, Utrecht University, in November 2015, under the guidance of Prof. Rob Bisseling and Associate Prof. Paul Zegeling, which culminated in this thesis. Her secondary duties at Utrecht university also included teaching; ranging from heading exercise sessions for undergraduates and tutorials to being solely responsible for teaching bachelor courses at University College Utrecht. She also assisted in one Mastermath course. During her PhD, Sehar participated in various international conferences. Besides this she was also secretary for SIAM student Chapter, Utrecht University for 2 years, and is currently member of the European Mathematical Society, European Women in Mathematics and Dutch-Flemish Scientific Computing Society. 


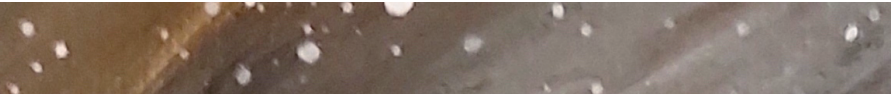

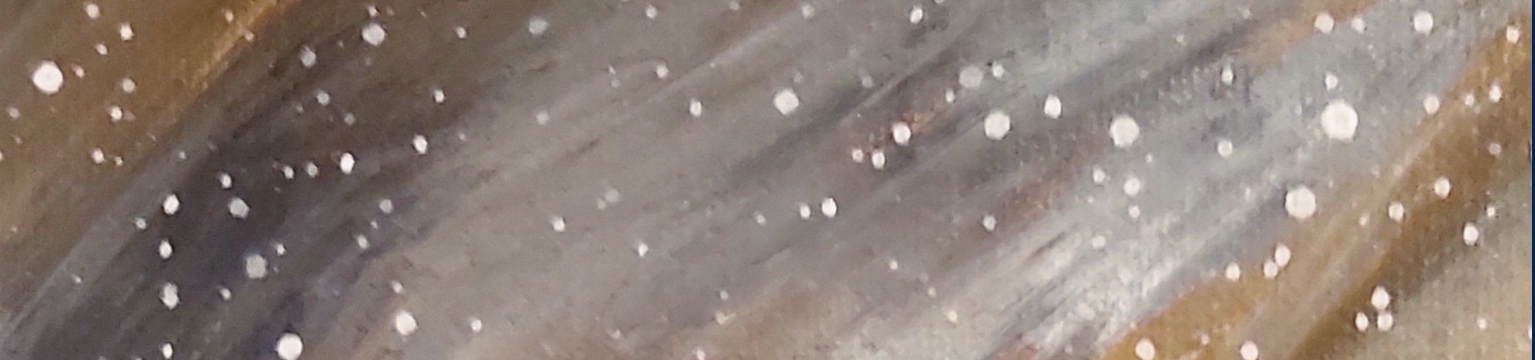

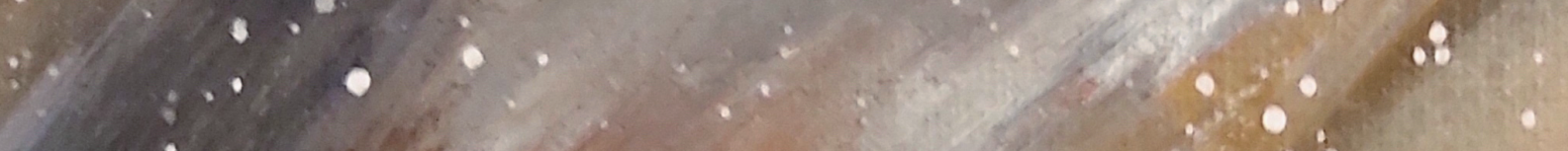

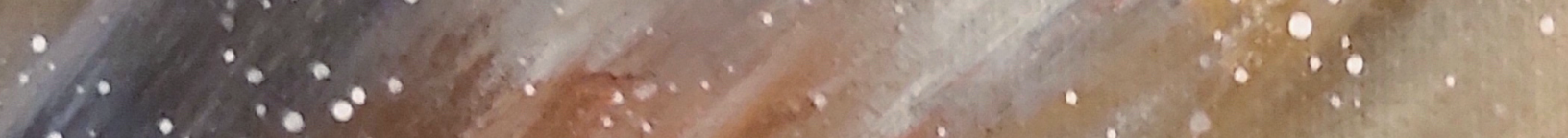

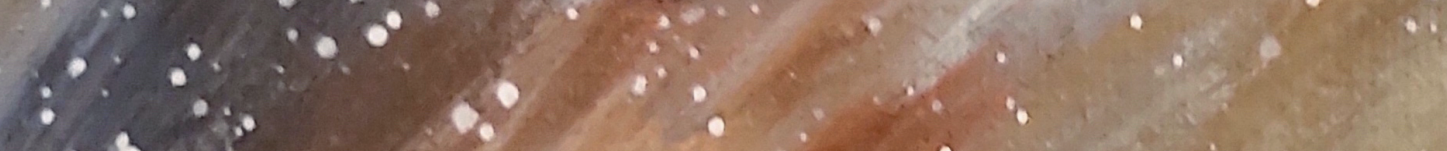

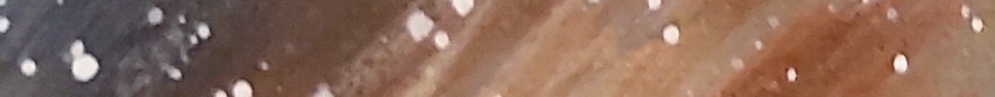
$0_{0}^{\infty} .0^{\circ}$

is
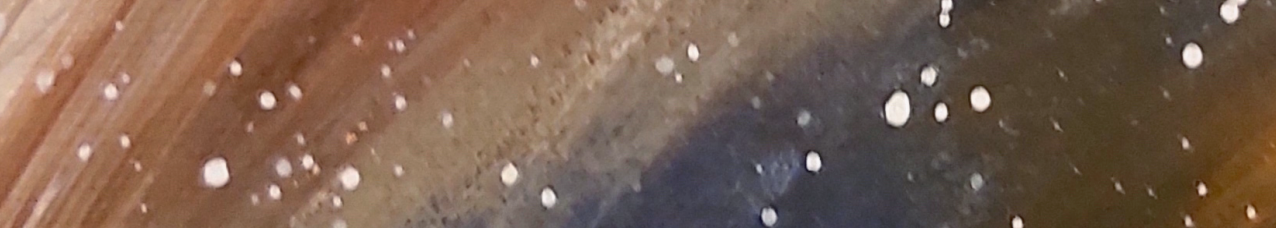

10
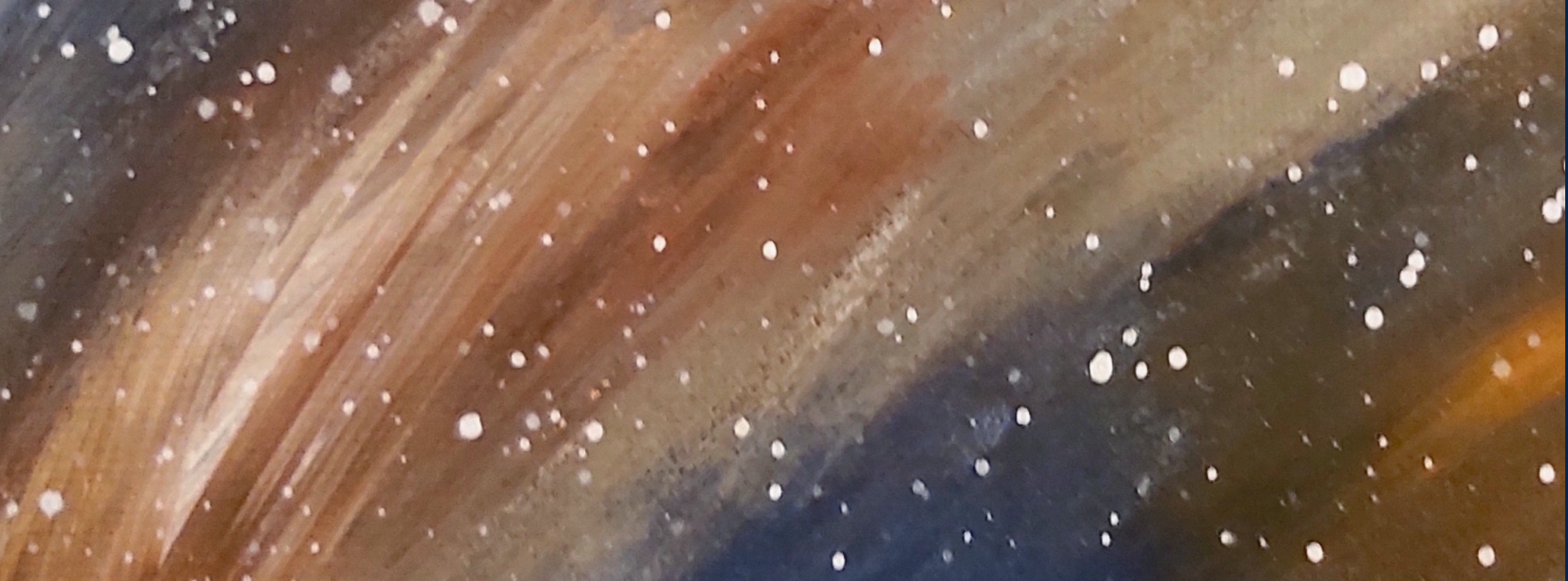

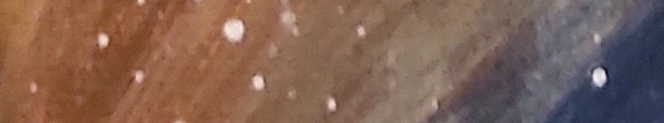

$i^{*} 0^{\infty}+\cdots$
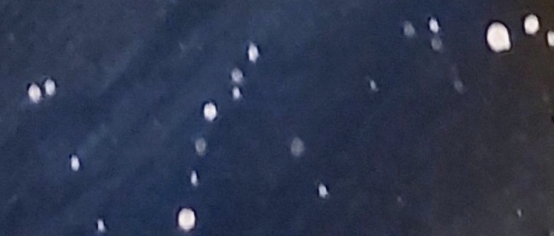

- 30.0
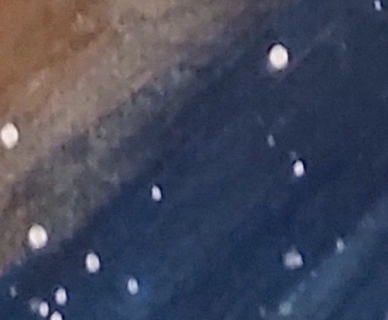

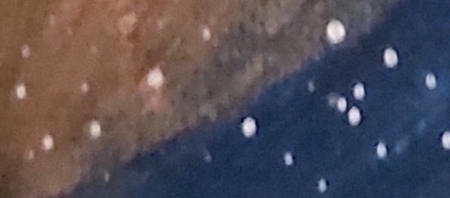
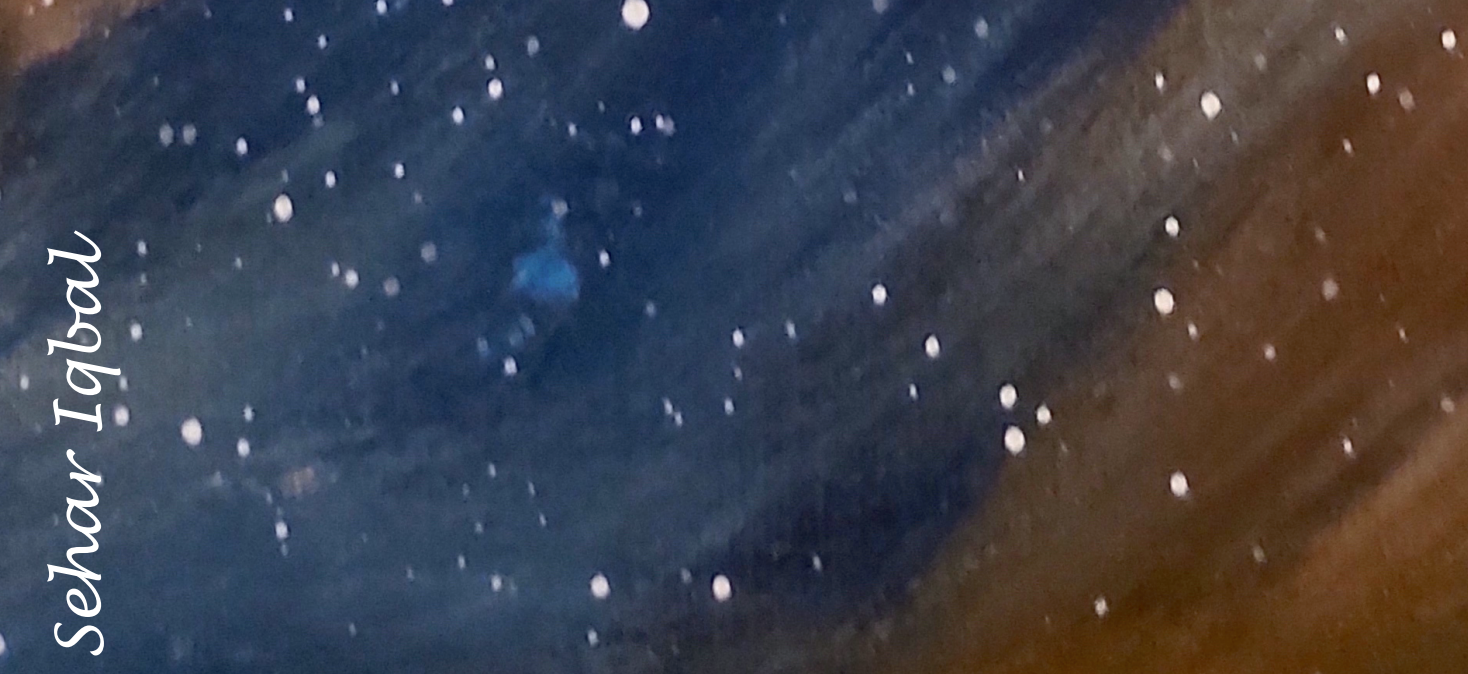